Progress Report on Wells Penetrating

Artesian Aquifers

In South Dakota

By R. W. DAVIS, C. F. DYER, and J. E. POWELL

GEOLOGICAL SURVEY WATER-SUPPLY PAPER 1534

Prepared in cooperation with the South Dakota State Water Resources Commission

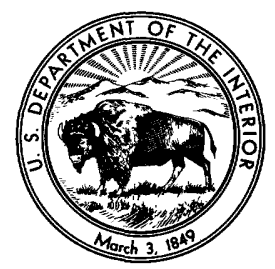




\section{UNITED STATES DEPARTMENT OF THE INTERIOR}

FRED A. SEATON, Secretary

\section{GEOLOGIGAL SURVEY}

Thomas B. Nolan, Director 


\section{CONTENTS}

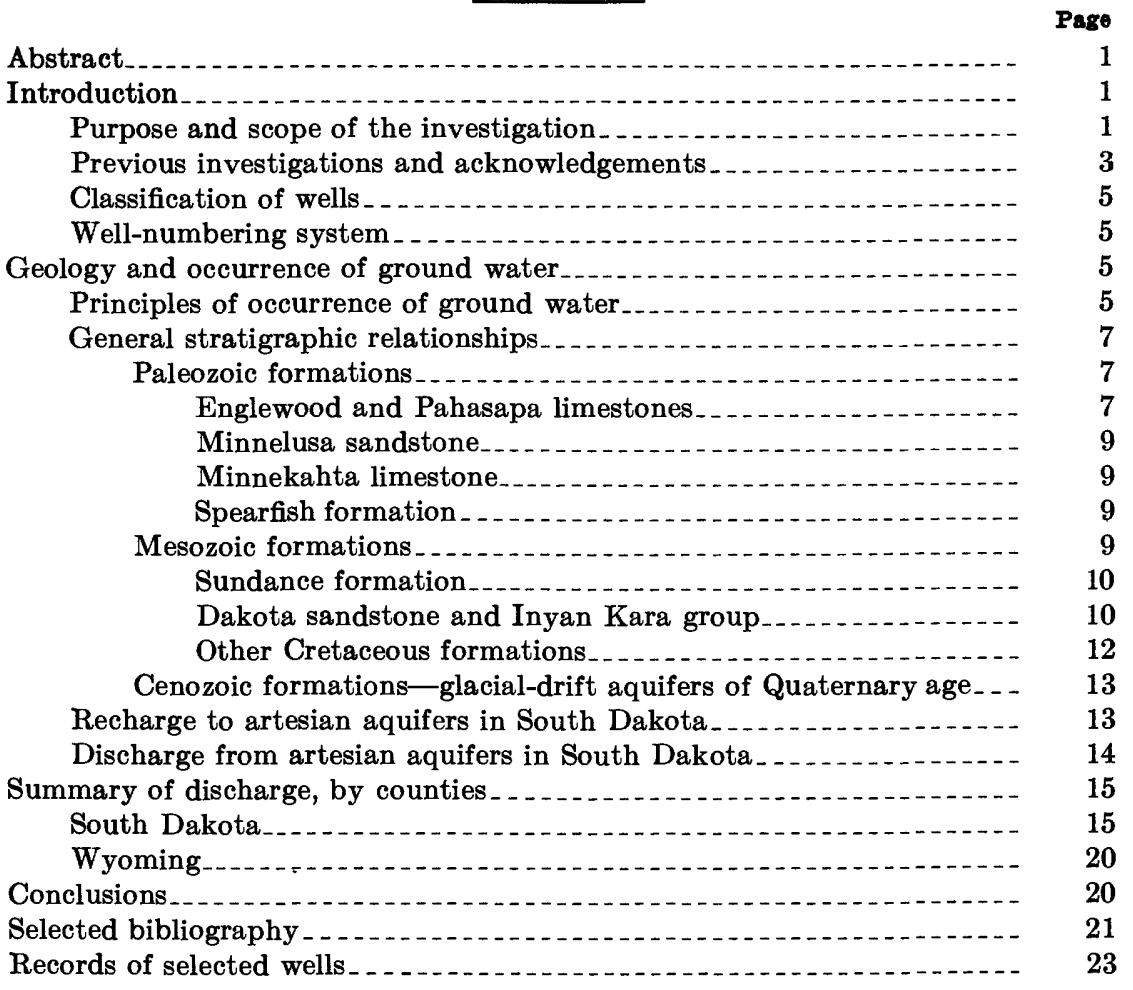




\section{ILLUSTRATIONS}

[Plates 1-3 in pocket]

Plate 1. Map of South Dakota showing location of wells having flows of more than $20 \mathrm{gpm}$.

2. Well index map of Brule County and parts of adjacent counties.

3. Map showing location of wells in Charles Mix and Gregory Counties.

4. $A$, Corroded "tee" taken from well 105-70-11dbbb1 in Brule County; $B$, Well A-9-3-27addb1, a former oil-test hole in Butte County :-

5. Two views of cratered well 100-71-26dbb1, Charles Mix

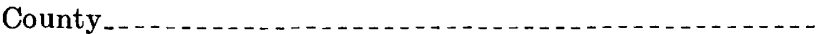

6. Unused well 98-68-31dbbb1, Charles Mix County, illustrating method used in measuring flow

7. $A$, Unrestrained well 95-65-35addd1, Charles Mix County; $B$, Small crater of unused well 97-68-29abc1, Gregory County -

8. Two views of uncontrolled well 102-72-5dcbe1, showing method of crater formation . . . . . . . . . . . . . . . . . .

9. Well 103-72-18adda1, a completely cratered well in Lyman County

Figure 1. Map of South Dakota showing counties visited and number of wells inventoried in each county

2. Sketch showing system of well identification

\section{TABLE}

TABLE 1. Records of selected artesian wells in South Dakota 


\title{
PROGRESS REPORT ON WELLS PENETRATING ARTESIAN AQUIFERS IN SOUTH DAKOTA
}

\author{
By R. W. Davts, C. F. Dyer, and J. E. Powell
}

A BSTRACT

Artesian aquifers underlie most of South Dakota and large areas in adjacent States. About 15,000 wells have been completed since 1881 in these aquifers within South Dakota. Many wells that originally flowed have ceased to flow and have been abandoned, and others have been equipped with pumps. Many thousands, however, continue to flow. This report presents data collected through June 1958 and includes records of 1,045 flowing and nonflowing artesian wells.

Sufficient information is not available at present (1958) to permit a detailed description of the geologic and hydrologic properties of artesian aquifers or their correlation in South Dakota. The description of the various aquifers given in this report is, therefore, necessarily a general one.

\section{INTRODUCTION}

\section{PURPOSE AND SCOPE OF THE INVESTIGATION}

Most of South Dakota and large areas in adjacent States are underlain by artesian aquifers. These aquifers yield water to flowing or pumped wells for stock-watering, domestic, municipal, and industrial uses.

Approximately 15,000 wells, most of them east of the Missouri River, have been completed in artesian aquifers in South Dakota since 1881. In 1955 the U.S. Geological Survey, in cooperation with the South Dakota State Water Resources Commission began an investigation to determine the present conditions of artesian wells and aquifers. This report presents data collected through June 1958 and includes records of 1,045 wells. Figure 1 shows the number of counties visited and the number of wells inventoried in each county.

The principal artesian aquifers that yield water to wells in South Dakota, together with their approximate ranges in depths of occurrence, in feet below the land surface, are the following geologic units: glacial drift (surficial deposits), Fox Hills sandstone (120-200), Niobrara formation (150-300), Codell sandstone member of the Carlile shale (300-400), Dakota sandstone and Fall River formation 


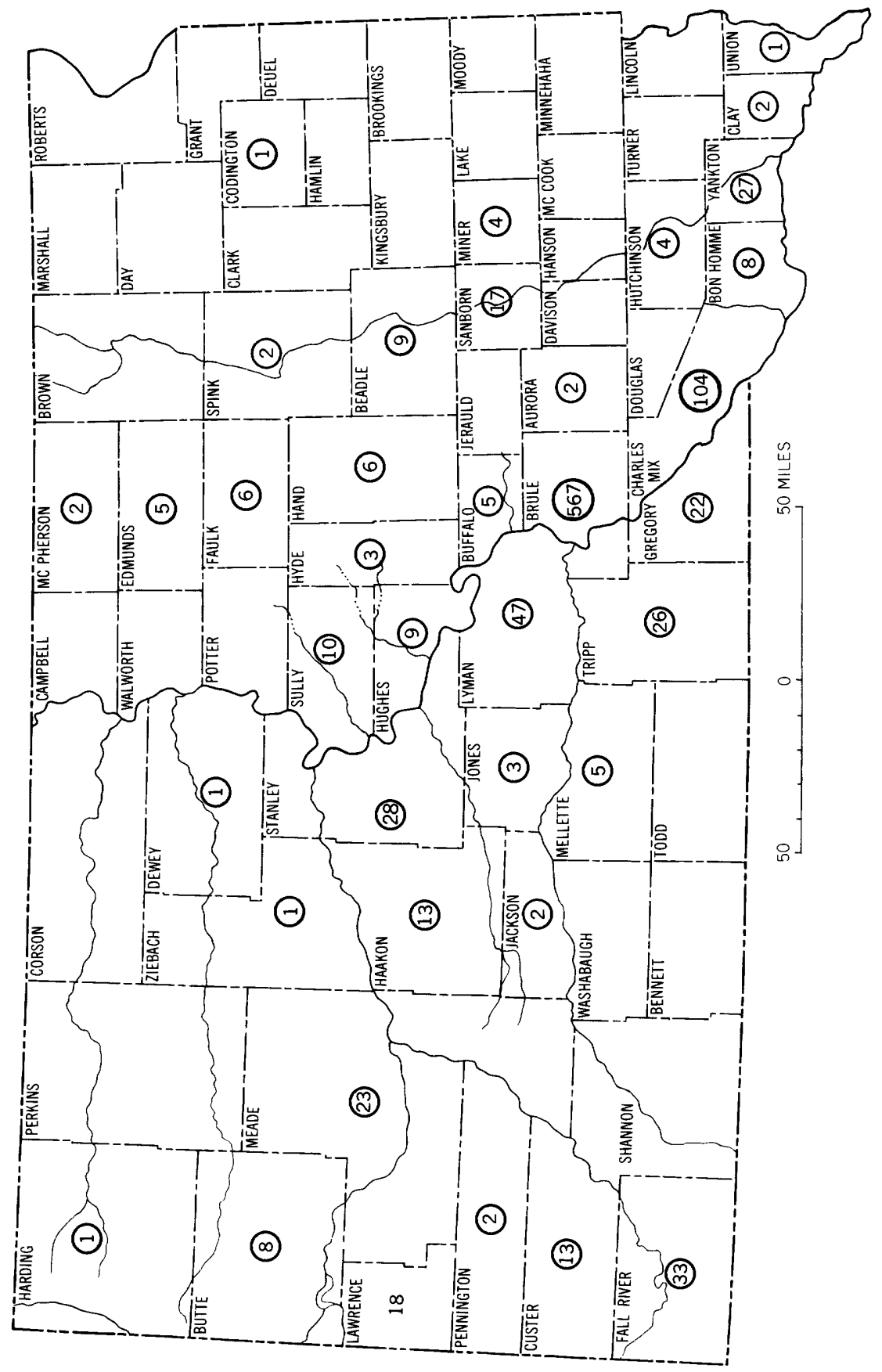

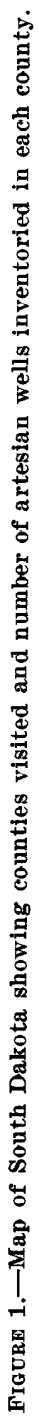


(600-1,300), Lakota formation (750-1,500), Sundance formation $(1,000-1,700)$, Minnelusa sandstone $(1,500-2,000)$, and Pahasapa limestone $(2,000-4,200)$. Of these units, the Dakota sandstone is the most widely used source of water.

Flowing wells in South Dakota discharge tremendous quantities of water. Many of the flowing wells inventoried in the valley of the Missouri River in Bon Homme, Brule, Buffalo, Charles Mix, Gregory, Lyman, and Yankton Counties are classed as "uncontrolled wells." The uncontrolled discharge from 46 such wells inventoried in these counties is approximately 16 million gallons per day (mgd). For comparison, the total flow from 3,054 wells known or presumed to tap the Dakota sandstone in 3,500 square miles of the James River valley in east-central South Dakota also is approximately $16 \mathrm{mgd}$.

The piezometric surface of an artesian aquifer is a hypothetical surface to which water will rise in or above a well that penetrates the aquifer. The piezometric surfaces of artesian aquifers in South Dakota have been declining since the first artesian well was drilled in the State in 1881. Continued drilling of wells that tap artesian aquifers and are allowed to flow unrestrained is hastening the day when all the wells will cease to flow.

Except for Brule County, the investigation was concerned chiefly with obtaining data on wells having flows of more than 20 gallons per minute (gpm). In Brule County, however, many low-yield flowing and pumped wells tap the same aquifer as uncontrolled wells having large flows. In order to obtain as many data as possible concerning the effects of large flowing wells on small pumped or flowing farm wells, all artesian wells in Brule County were inventoried.

Sufficient information was not available at the time the report was prepared (1958) to permit a detailed description of the geologic and hydrologic properties of artesian aquifers in South Dakota. The general physical properties of the principal artesian aquifers in the State are given in the following table. All aquifers penetrated by wells listed in the table are discussed in the text.

\section{PREVIOUS INVESTIGATIONS AND ACKNOWLEDGMENTS}

Earlier geologic and ground-water investigations of broad scope were made in South Dakota by Todd $(1895,1896)$, Darton (1896, 1905, 1909), and Rothrock (1943). The report of the South Dakota State Planning Board (1937) contains general information on artesian wells and maps showing the location of these wells throughout the State. Information on flowing and nonflowing wells is presented in county reports by the South Dakota State College Extension Service $(1940)$. 


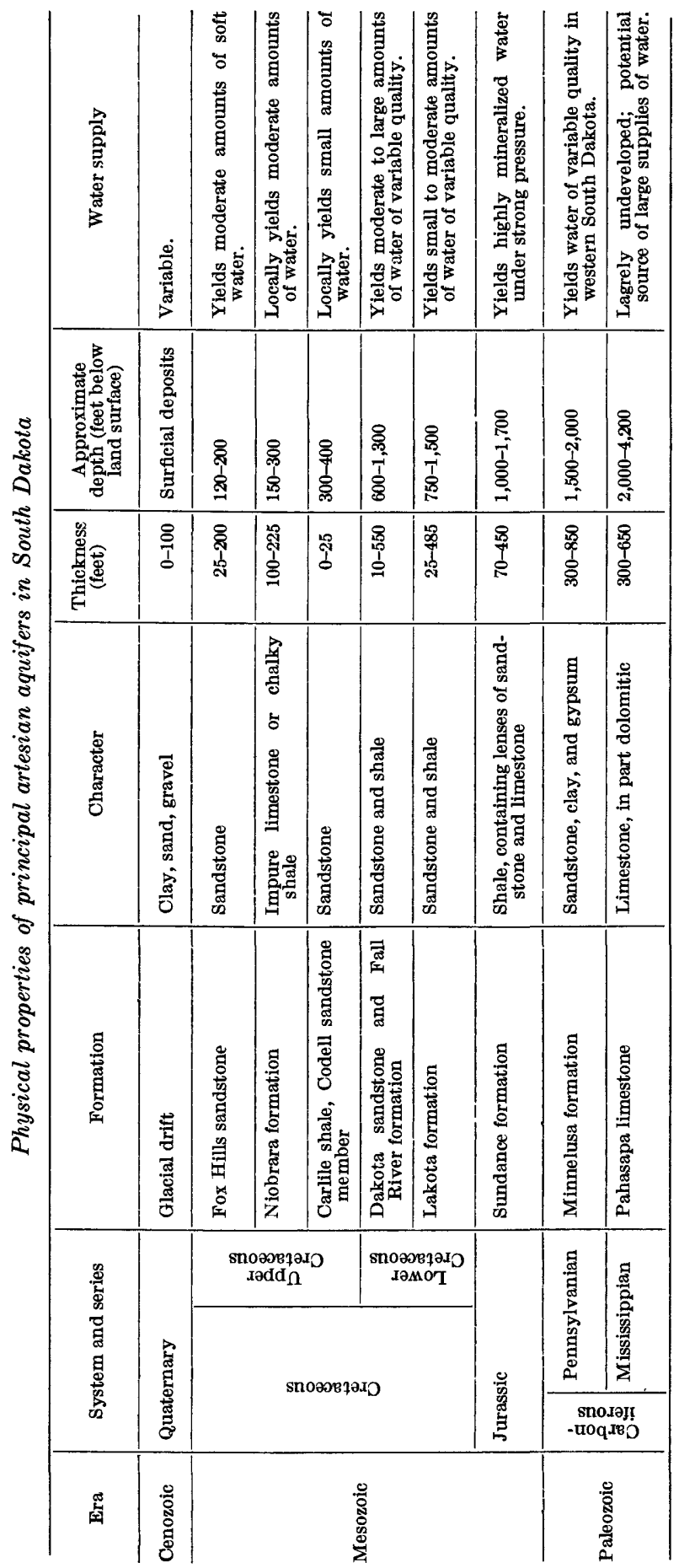


The cooperation of the many farmers and ranchers throughout the State whose properties were visited during the course of the field investigation is gratefully acknowledged.

\section{CLASSIFICATION OF WELIS}

In this report the term "uncontrolled," when applied to a well, means that the well is flowing out of control and indicates that it could not be easily brought under control. Whereas the terms "not controlled" or "unrestrained" mean that the well is flowing at full capacity but its flow might be restricted by the addition of a valve at the well head. The term "uncontrolled" is used exclusively to refer to cratered wells having no visible casing or to wells in which the water is rising outside, as well as inside, the casing.

\section{WELL-NUMBERING SYSTEM}

Wells are numbered in accordance with the United States Bureau of Land Management's system of land subdivision. The first numeral of a well designation indicates the township; the second, the range; and the third, the section of the township in which the well is situated. Lowercase letters after the section number indicate the location of the well within the section: the first letter denotes the 160 -acre tract; the second, the 40-acre tract; the third, the 10-acre tract; and the fourth, the $21 / 2$-acre tract. The letters $a, b, c$, and $d$ are assigned to the tracts in a counterclockwise direction, beginning in the northeast corner of each tract. The number of lowercase letters indicates the accuracy of the well location; if the well can be located within a $21 / 2$-acre tract, four letters are shown in the well number. To distinguish between two or more wells situated within the same tract, consecutive numbers, beginning with 1 , are added as a suffix to each well number. Well numbers preceded by the capital letters $A$ and $D$ designate wells in the northeast and southeast quadrants of the Black Hills meridian and base-line system. Well numbers not preceded by capital letters designate wells in the fifth and sixth principal meridian and base-line systems. 'The method of designating the location of wells is shown in figure 2.

\section{GEOLOGY AND OCCURRENCE OF GROUND WATER}

\section{PRINCIPLES OF OCCURRENCE OF GROUND WATER}

Practically all ground water is derived from precipitation. Rain and meltwater from snow enter the ground by direct percolation or by percolation from streams and lakes that lie above the water table. 


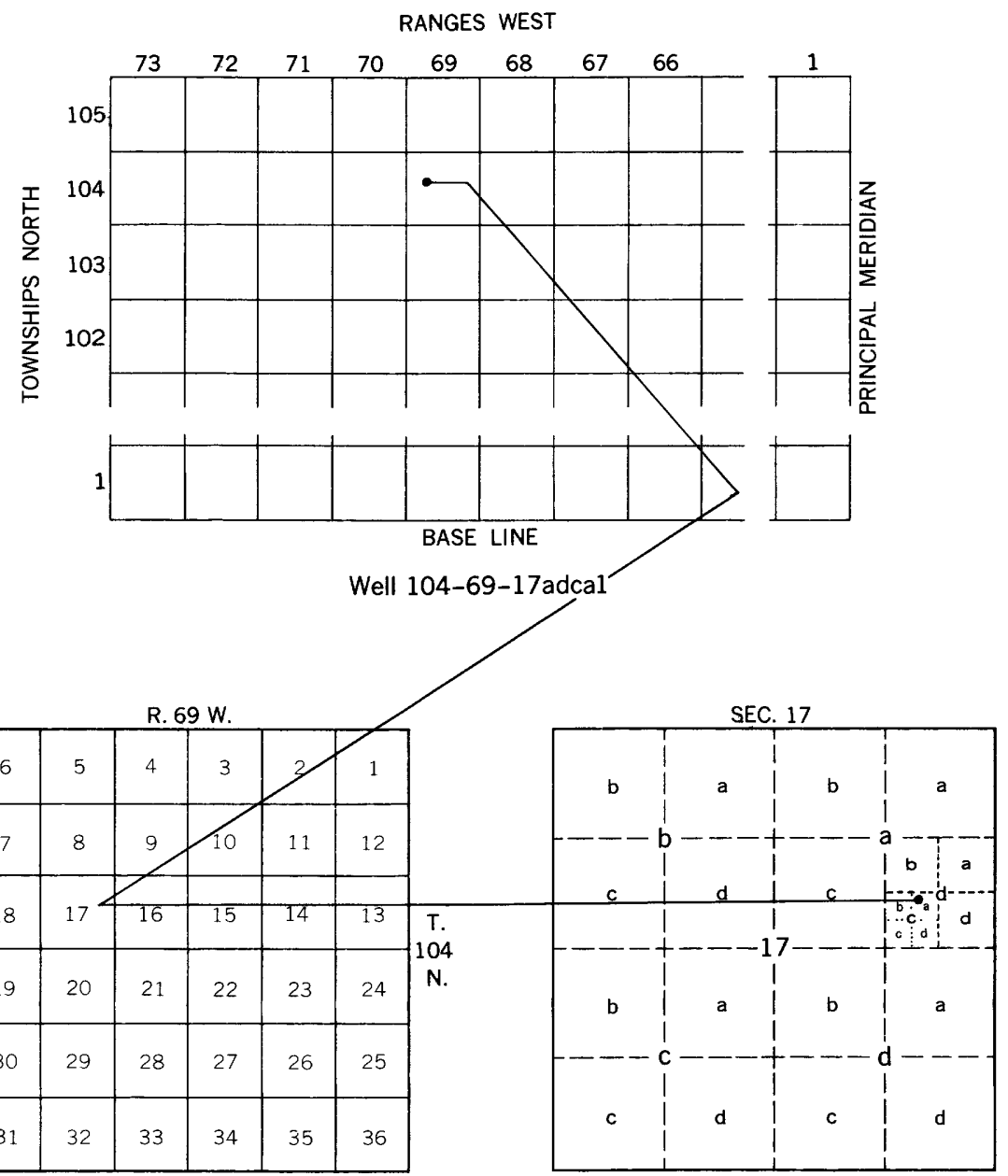

Figure 2.-Sketch showing system of well identification. 
Ground-water discharge occurs through transpiration by plants, by evaporation from the soil where the ground-water level is at or near the land surface, by seepage into surface-water bodies, and by discharge from wells or springs.

Any rock formation or stratum that will yield water in sufficient quantity to be important as a source of supply is called an aquifer (Meinzer, 1923, p. 52). Water moving in an aquifer from recharge areas to discharge areas may be considered to be in "transient storage."

The amount of water that a rock can hold is determined by its porosity. Unconsolidated materials, such as clay, sand, and gravel generally are more porous than consolidated rocks, such as sandstone and limestone; however, some consolidated rocks are very porous.

The frictional resistance to the movement of water through pore spaces that are relatively large, such as those in coarse gravel, is not great and the material is said to be highly permeable. However, the resistance to the movement of water through small pore spaces such as those in clay or shale may be very great and the material is said to be relatively impermeable or to have low permeability.

If the water in an aquifer is not confined by overlying impervious strata, it is said to be under water-table conditions. Under these conditions, the water can be obtained from the aquifer by lowering the water level, for example, by pumping from a well.

Water is said to be under artesian conditions if it is confined in an aquifer by an overlying relatively impermeable stratum. Under artesian conditions, the water in a well penetrating the aquifer will be raised by hydrostatic pressure to a level above the top of the aquifer. As with water under water-table conditions, water can be obtained from the aquifer if the water level is lowered by pumping or if the hydrostatic pressure is lowered by natural flow. However, when water under artesian conditions is released from storage, as it is in all the aquifers described in this report, the aquifer remains saturated and yields water from storage by the expansion of the water and compression of the aquifer as the head is decreased. Gravity drainage, such as occurs with water under water-table conditions, does not occur as long as artesian conditions exist.

\section{GENERAL STRATIGRAPHIC RELATIONSHIPS}

PALEOZOIC FORMATIONS

\section{ENGLEWOOD AND PAHASAPA LIMESTONES}

The Englewood and Pahasapa limestones of Mississippian age crop out around the Black Hills and may underlie much of western South Dakota. Inasmuch as drill cuttings from the Pahasapa and Engle- 
wood limestones cannot easily be distinguished from one another, it is common practice to list both as the Pahasapa limestone in drillers' $\operatorname{logs.}$

Much of the Pahasapa is a fine-grained massive light-gray to buffcolored limestone or dolomite containing numerous caverns. Many of the caverns in this formation in the Black Hills are lined with calcite crystals. Near the Black Hills the Pahaspa ranges in thickness from 300 to 630 feet. The formation thins eastward, and in the central and southwestern parts of the State it pinches out against Precambrian quartzite, schist, and granite.

The Pahasapa yields water to wells in Fall River, Pennington, Meade, Hughes, Butte, and Dewey Counties. If a well penetrates a cavernous part of the formation, large supplies of water under high pressure commonly are obtained (well A-9-3-27addb1). Where wells do not intercept cavernous passages, they are essentially dry holes. A well drilled on a structural dome at Bear Butte in 1956 (well A-6-6-19 bbcd1) obtained a large flow from the Minnelusa sandstone; however, this well penetrated more than 400 feet of the underlying Pahasapa limestone with no increase in flow.

\section{MINNELUSA SANDSTONE}

The Minnelusa sandstone of Pennsylvanian age consists mainly of white and reddish calcareous sandstone; locally, limestone beds occur in the middle and lower parts of the formation. The base of the formation is characterized by bright-red shale and thin layers of white limestone. The formation ranges in thickness from 300 to 850 feet in the outcrops around the Black Hills. It thins to the east and northeast but the areal extent of the formation in those directions is indefinite. Probably the Minnelusa pinches out against the Precambrian rocks, roughly along a line extending northeastward through the southeastern part of Hyde County and the northwestern part of Hand County (Erickson, 1954, p. 40).

Near the Black Hills the Minnelusa yields abundant supplies of water to flowing wells. In the central part of the State a coarse unconsolidated sand that is equivalent in age to the lower part of the Minnelusa locally yields large supplies of water under high pressure. Erickson (1954, p. 40) refers to this sand as the Millstone grit. A well at the Pierre airport, reported to tap this sand, flowed $600 \mathrm{gpm}$ and was sealed off for fear that the flow might become uncontrollable. Railroad and municipal wells at Edgemont (wells D-9-2-1aabd2 and D-9-2-1acdb1) were considered by Darton $(1918$, p. 98$)$ to tap the Deadwood formation of Cambrian age, but information obtained from more recent drilling indicates that these wells tap the Minnelusa sandstone and perhaps part of the Pahasapa limestone. 
Other wells inventoried tap the Minnelusa sandstone in Butte, Lawrence, Custer, and Meade Counties.

\section{MINNEKAHTA IIMESTONE}

The Minnekahta limestone of Permian age is dense thin bedded, light pink gray to dark lavender and very hard. Its thickness ranges from 30 to 50 feet at the outcrop in the Black Hills area. The areal extent of the Minnekahta limestone is not known.

Ordinarily the Minnekahta is not considered to be an aquifer, but at Hot Springs and Cascade Springs in the southern Black Hills, and northwest of Spearfish near the South Dakota-Wyoming border, the Minnekahta yields large amounts of water of good quality under artesian conditions. However, this water is probably moving upward from the Minnelusa and Pahasapa formations along faults and other fissures.

The occurrence of a large supply of water in the Minnekahta limestone near Piedmont (wells A-3-6-15ba1 and A-3-6-15ba2) also may be attributed to the upward movement of water along fissures resulting from the flexing and folding of Paleozoic and Mesozoic strata along the eastern flanks of the Black Hills uplift.

\section{SPEARFISH FORMATION}

The Spęarfish formation, of Permian and Triassic age, consists of red sandy shale and soft red sandstone and siltstone containing masses and stringers of gypsum. The thickness of the formation at its outcrop in the Black Hills ranges from 350 to 700 feet. The formation thins rapidly to the east, but its eastern boundary has not been definitely established. In general, the Spearfish formation is not very permeable. Locally along the outcrop it contains highly mineralized water.

In T. 7 N., R. 1 E., in Lawrence County, several artesian wells are reported to obtain large flows of water from the Spearfish formation. Well A-7-1-20cddd1 is reported to obtain water from a cavity at the base of the Spearfish. This well is in an area of artesian springs northwest of the city of Spearfish, where the possible leaching of gypsum deposits of the Spearfish formation by movement of water to springs, may have created the cavities that now yield water to wells. No other artesian wells within the State are reported to tap the Spearfish formation.

\section{MESOZOIC FORMATIONS}

Mesozoic formations contain the most widely tapped artesian aquifers in South Dakota. The Dakota sandstone in central and eastern South Dakota is the most productive artesian aquifer in the State. 
Stratigraphic nomenclature in this area has become complicated by the introduction of formation names formerly used only in the Black Hills and other western areas. The terms Fuson shale, Lakota sandstone, Sundance formation, and "Detrital" zone have been applied to strata believed by some authors to lie below or in the lower part of the strata commonly considered to form the Dakota sandstone. The problem of correlation and nomenclature, far from being a purely academic matter, is of importance to interpretation of the subsurface stratigraphy and determination of the quantity and quality of available ground-water supplies in South Dakota.

\section{SUNDANCE FORMATION}

The Sundance formation of Jurassic age is composed of gray-green shale and small lenses of sandstone and limestone, bluff sandstone, and red sandstone and shale. At the outcrop in the Black Hills area the thickness of the formation ranges from 70 to 450 feet. Water in the Sundance formation is reported to be highly mineralized, although locally, near its outcrop, the formation yields less mineralized water that is suitable for domestic use. Water in the Sundance formation in central and northern South Dakota occurs under high artesian pressure and is very corrosive. Consequently only strong, corrosionresistant casings and screens can be successfully used in wells that obtain water from this formation.

In central and north-central South Dakota a sandstone, or a rock reported as "grit", that is deeper than the Dakota sandstone has been tapped by wells that yield water under high pressure. This rock has been logged as the Sundance formation by Baker $(1947$, p. 6,10$)$ and by Erickson (1954, p. 38 and 1955, p. 7). Most drillers $\log$ this deeper aquifer as the Sundance formation. Glauconite, a mineral not common in the younger Cretaceous aquifers, in the drill cuttings is generally indicative of this aquifer or facies. The correlation of the sandstone with the Sundance formation of the Black Hills has not been established in the field. Gries (1954, p. 447) stated that the Skull Creek shale, containing a thin but conspicuous glauconitic zone, can be traced to east-central South Dakota and has been reported as Sundance shale in that area.

Erickson (1955, p. 16) described a "detrital" zone that underlies the Sundance formation, but none of the wells listed in table 1 are reported to tap this aquifer.

\section{DAKOTA SANDSTONE AND INYAN KARA GROUP}

In eastern South Dakota, a fine- to medium-grained sandstone with interbedded silt and clay, underlies the Graneros shale. It has been known as the Dakota sandstone since early geologic studies were made 
at its outcrop in northeastern Nebraska, and is now considered to be Late Cretaceous in age. The Dakota has yielded water to thousands of flowing and nonflowing artesian wells throughout the Dakotas and adjacent States; wells tapping this aquifer are especially abundant in eastern and central South Dakota.

In the outcrops in the Black Hills, the Inyan Karan group includes the Lakota and Fall River formations. The Fall River formation of Early Cretaceous age underlies the Graneros shale. The Fuson member of the Lakota formation, containing shale and minor sandstone units, underlies the Fall River formation and separates it from the rest of the Lakota formation. The Fall River and Lakota formations are lithologically similar to the Dakota sandstone of eastern and central South Dakota and are important aquifers in the western part of the State. The total thickness of the Inyan Kara group ranges from 45 to 900 feet near the Black Hills.

Correlation of the Lakota and the Fall River with the Dakota from their respective areas of outcrop to central and eastern South Dakota is uncertain at present. Gries $(1954$, p. 447$)$ reported that the Dakota sandstone of eastern and central South Dakota is the time equivalent of parts of the Graneros shale and that the Fall River formation may be traced eastward into the lower part of the Dakota. According to this interpretation, the Lakota through Graneros sequence of beds is a western facies of the Dakota sandstone, that is, the Dakota sandstone of the east grades westward into shale and tongues of sandstone that have been given different names at their outcrops in the Black Hills; the eastern and western facies are both buried beneath younger formations.

In central and eastern South Dakota there are two aquifers in the Dakota. The deeper aquifer, commonly called "second flow" by residents and drillers, has been correlated by some authors with the Lakota sandstone on the basis of a reportedly traceable key horizon in the overlying Fuson shale. Baker $(1948$, p. 2) reported that a zone of manganese-bearing pellets at the top of the Fuson shale can be traced from its outcrop at Sergeant Bluff south of Sioux City, Iowa, westward to the Black Hills. He concluded, therefore, that the Fuson shale, the underlying Lakota sandstone, and the overlying Dakota sandstone were continuous throughout the Dakota artesian basin and that the Dakota sandstone of eastern and central South Dakota could be correlated with the Fall River sandstone in the Black Hills area. Gries $(1954$, p. 448$)$, however, believed that the pellets are a product of the environment under which the clays were deposited and are not necessarily an index to the presence of the Fuson formation. He based this belief on the fact that in wells in the central part of the State, where both the Dakota and the Fall River are well defined, a zone of 
pellets occurs in association with the buff or varicolored clay in each sandstone.

Additional field studies, test drilling, and quality-of-water studies would be necessary to establish definitely the stratigraphic relationship of Lower Cretaceous aquifers in eastern and central South Dakota to those in the western part of the State.

In this report the names "Lakota" and "Fall River" are used for areas near the Black Hills (the name "Fall River" is used mainly for the area within several miles of the outcrop of the formation), whereas for central and eastern South Dakota the names "Dakota" and "Lakota" are used.

\section{OTHER CRETACEOUS FORMATIONS}

Other formations of Cretaceous age that yield water under artesian conditions are the Codell sandstone member of the Carlile shale, the Niobrara formation, and the Fox Hills sandstone.

The Codell sandstone member occurs near the top of the Carlile shale. It consists of fine angular to subangular quartz sand, a few of the grains being frosted or etched. The extent of the Codell sandstone member is not definitely known, but it has been reported to be present in wells in an area extending from Pennington County in western South Dakota to the vicinity of Huron in the east-central part of the State. Elsewhere the Codell grades into clay or is missing.

The Niobrara formation is an impure limestone or chalky shale. It underlies the entire State with the exception of the Black Hills area in the western part, the vicinity of the ridge of Sioux quartzite in the extreme eastern part, and areas where it was removed by preglacial erosion.

In the western part of South Dakota the Niobrara formation ranges in thickness from 100 to 225 feet. The formation thins to the east and pinches out against the Sioux quartzite ridge in eastern South Dakota.

Several wells in Brule and Charles Mix Counties in southeastern South Dakota are reported to tap the Codell sandstone member and several other wells tap the Niobrara formation. Water in the Codell and the Niobrara is not under sufficient pressure to flow at the land surface in these counties, but both units are important sources of water for pumped wells in the east-central and southeastern parts of the State.

The Fox Hills sandstone is separated from the Niobrara formation by 1,200 to 2,500 feet of the Pierre shale. The Fox Hills is composed of grayish-white to yellow sandstone and crops out north and northeast of the Black Hills in Butte, Meade, and Ziebach Counties. In Harding County, well A-17-4-16bdc1 obtains a small flow of water from the Fox Hills( ?) sandstone, but the formation is not considered 


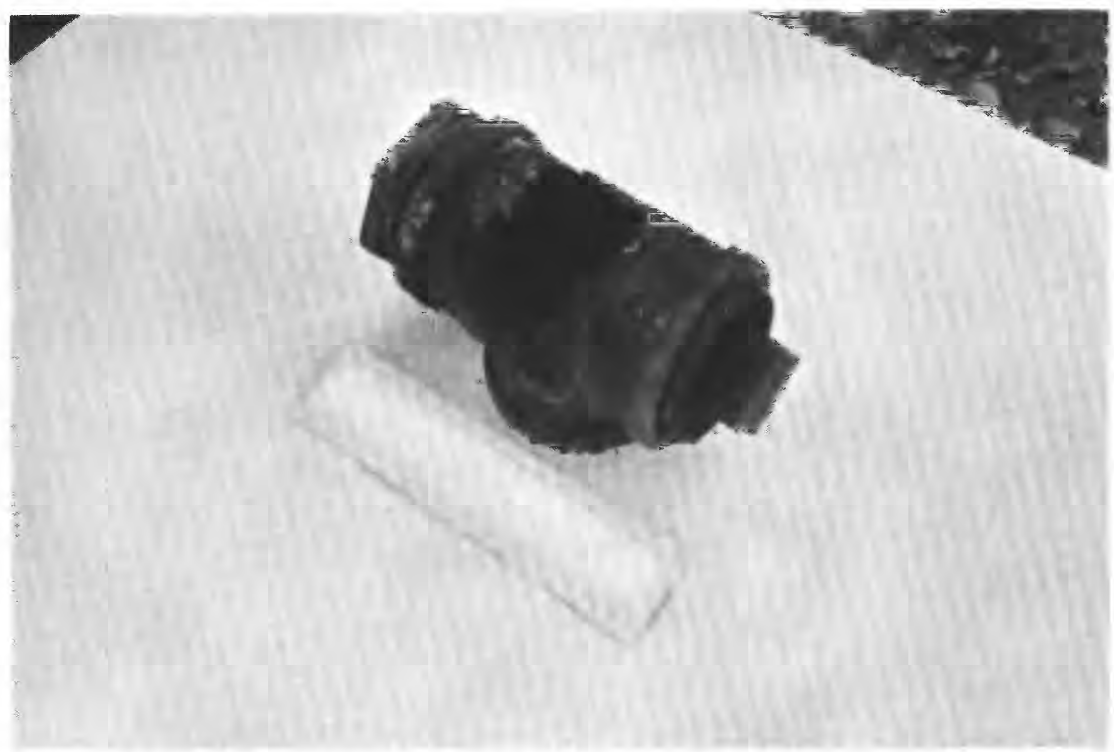

A. CORRODED "TEE" FROM CASING OF WELL 105-70-11dbbbI IN BRULE COUNTY

This fixture had been on the casing approximately 3 years. Scale is indicated by 6-inch rule.

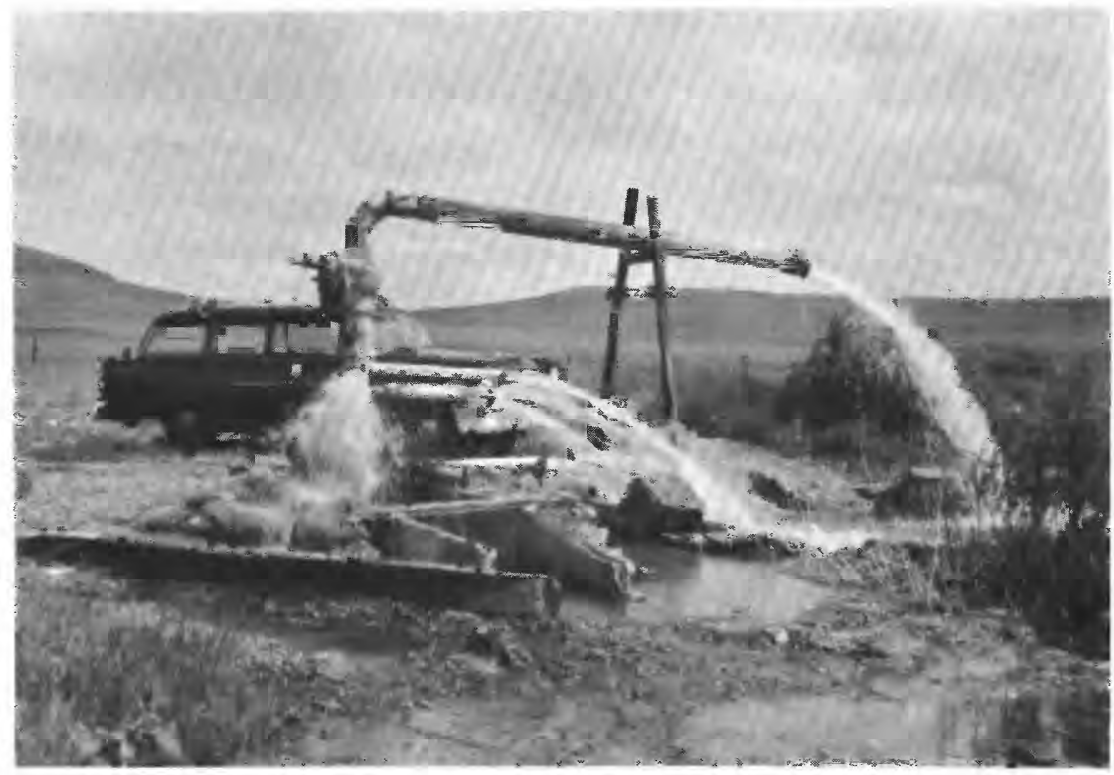

B. UNUSED WELL A-93-27addbl, A FORMER OIL-TEST HOLE IN BUTTE COUNTY Flow is reported to be $2,000 \mathrm{gpm}$, from the Minnelusa and Pahaspa formation. 


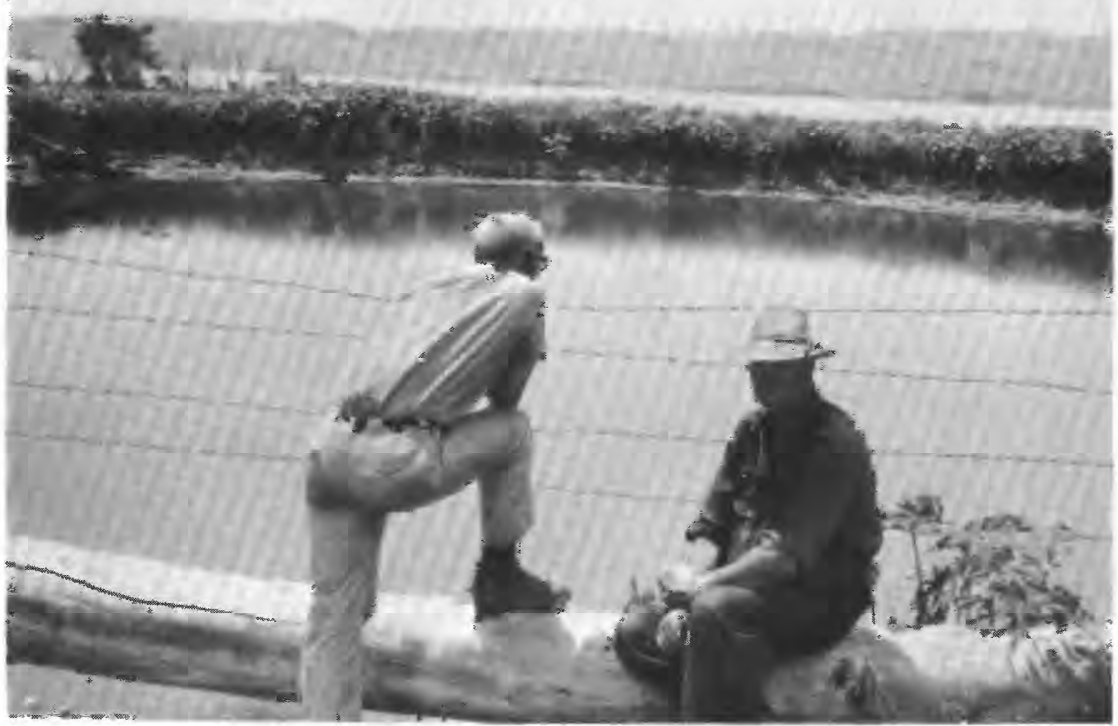

A. POND FORMED BY BULLDOZING AN EMBANKMENT AROUND THE WELL SITE

The casing of the well is now completely destroyed and the approximate location of the former well head is slightly to the left of the midpoint of the right margin of the photograph.

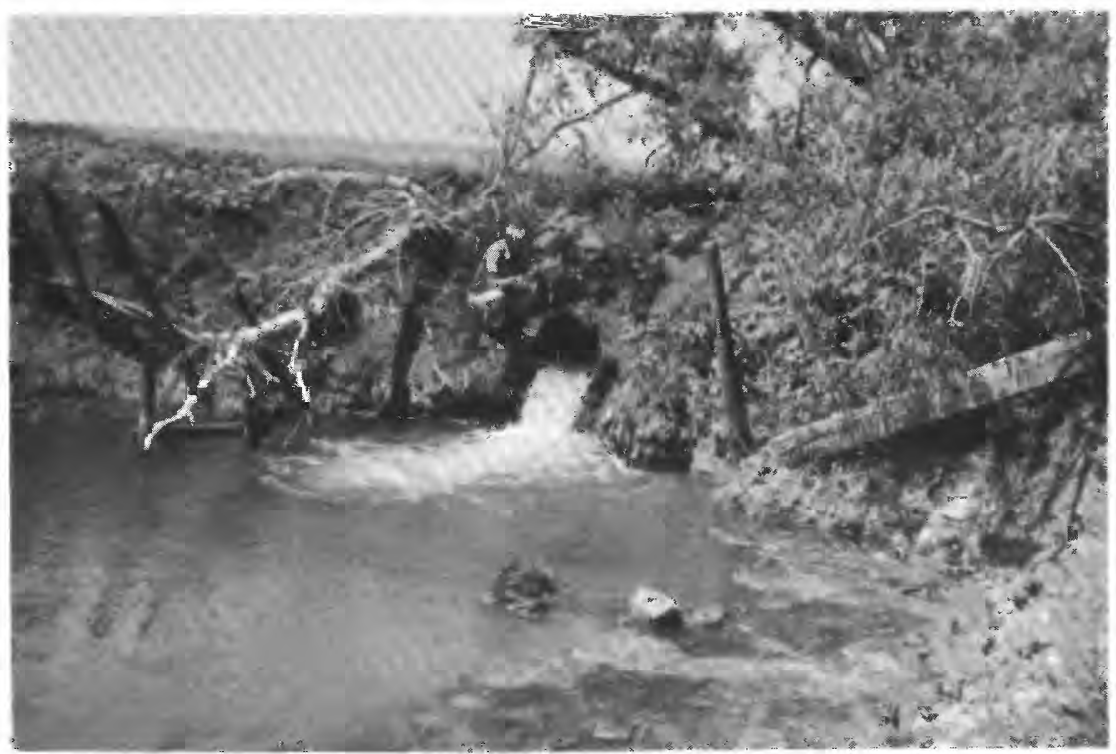

B. FLOW DISCHARGING THROUGH CULVERT PIPE IN EMBANKMENT AROUND POND Almost none of the water of the 1,500 gpm flow discharging through the 29-inch culvert pipe is being used. 


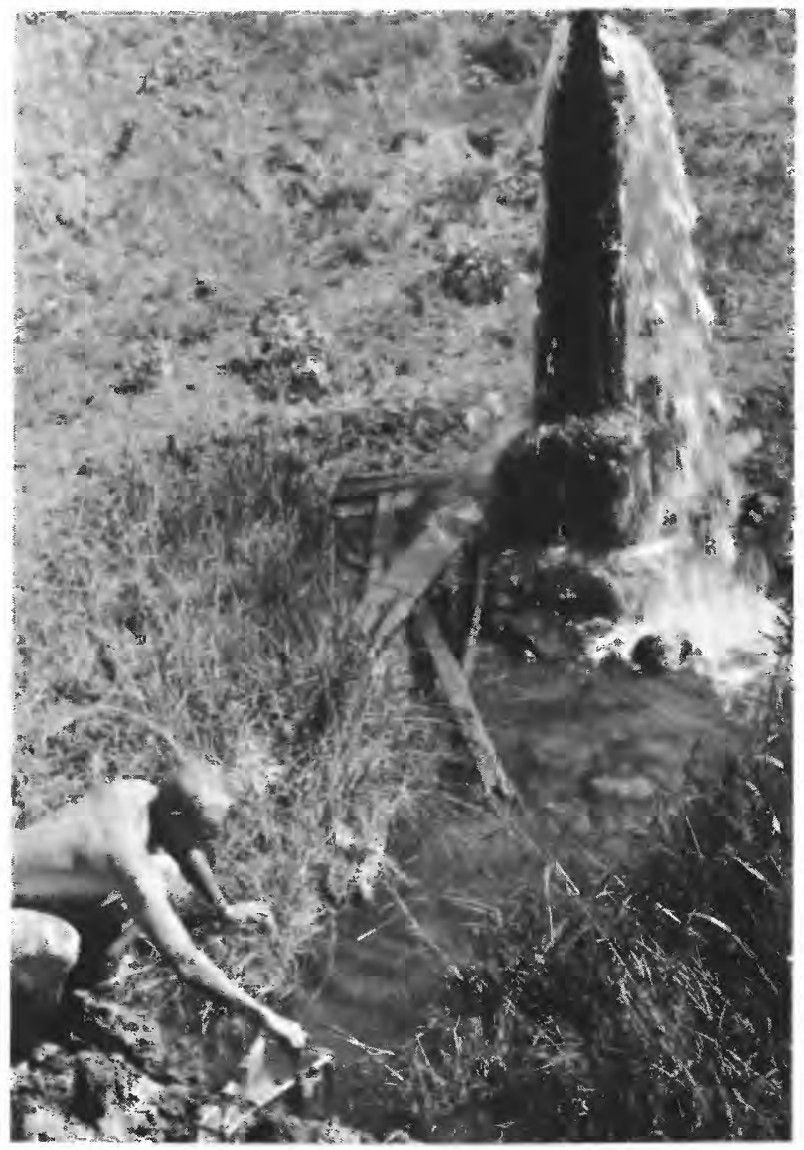

WELL 98-68-31dbbb1 IN CHARLES MIX COUNTY

View of unused well, illustrating method of measuring flow from unrestrained wells by use of a Parshall flume. Flow of $247 \mathrm{gpm}$ discharges from 6-inch opening at top of the casing, approximately 8 feet above the land surface. 


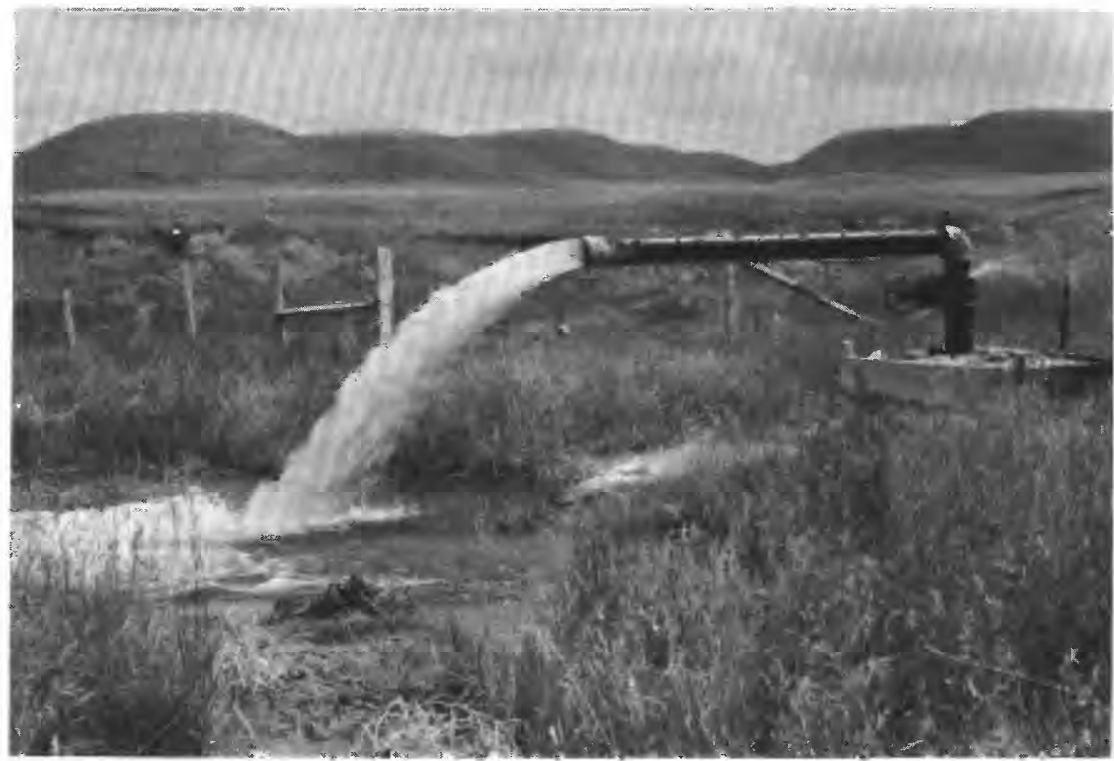

A. UNRESTRAINED WELL 95-65-35addd1 IN CHARLES MIX COUNTY

The well casing is reported to vibrate strongly when flow is restricted. Note deterioration of casing immediately above valve.

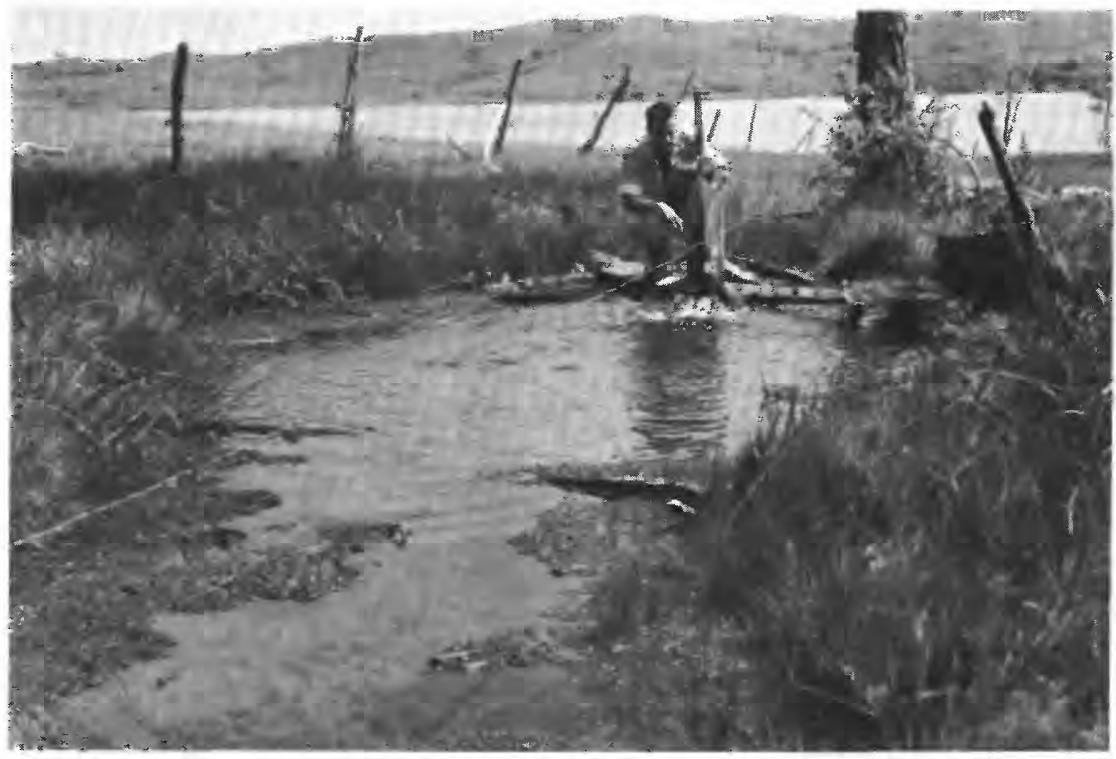

\section{B. SMALL CRATER OF UNUSED WELL 97-68-29abcl IN CHARLES MIX COUNTY}

The flow, about $600 \mathrm{gpm}$, is discharged over land surface in lower left of photograph and eventually enters Fort Randall reservoir, visible in backgroụnd. The stake in front of kneeling man marks the location of the badly corroded casing. 


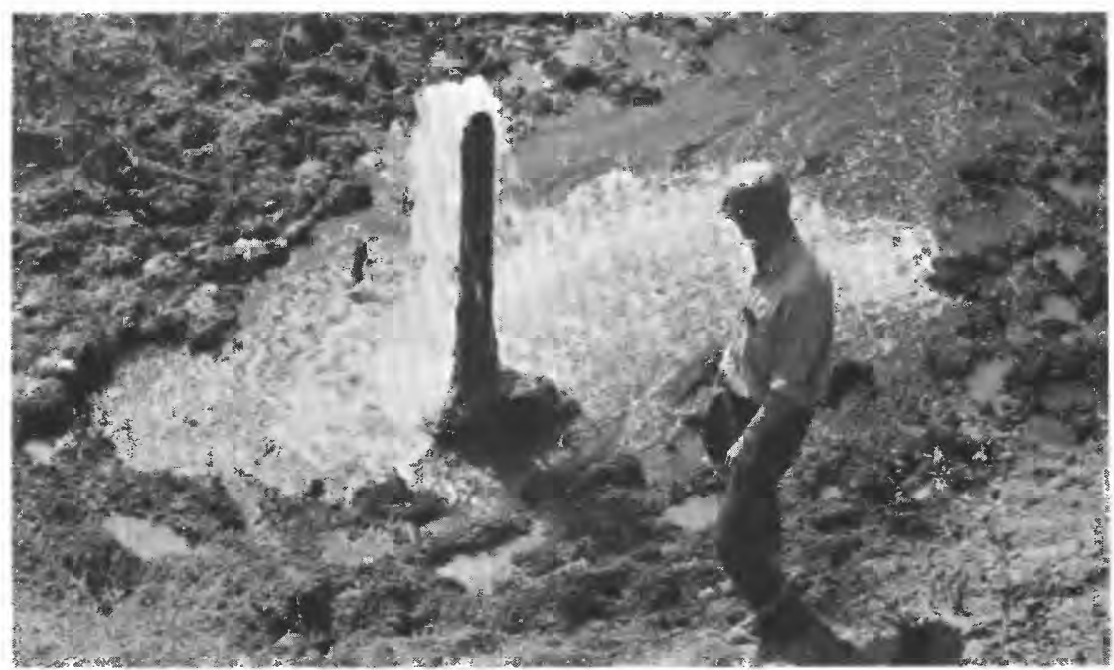

A. BOTTOM OF SMALL CRATER

Note break in casing about 1 foot above base; flow of well is $177 \mathrm{gpm}$.

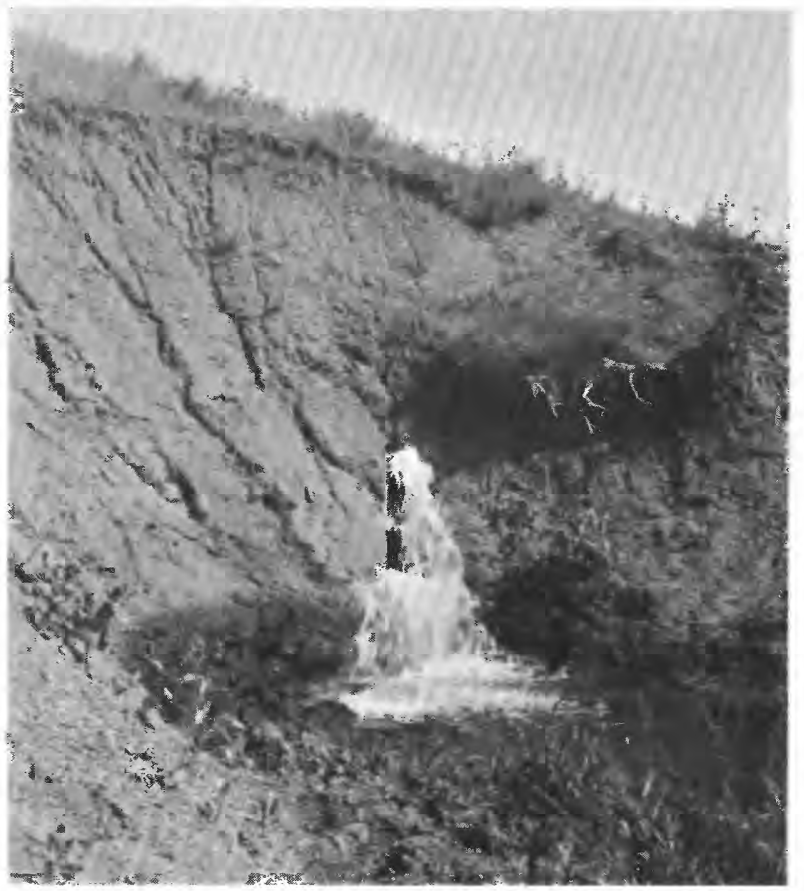

B. CHARACTERISTIC SLUMPING OF PIERRE SHALE ON SLOPE OF SMALL CRATER View to the northeast; slumping at upper right.

VIEWS OF UNCONTROLLED WELL 102-72-5dbc1, SHOWING METHOD OF CRATER FORMATION 


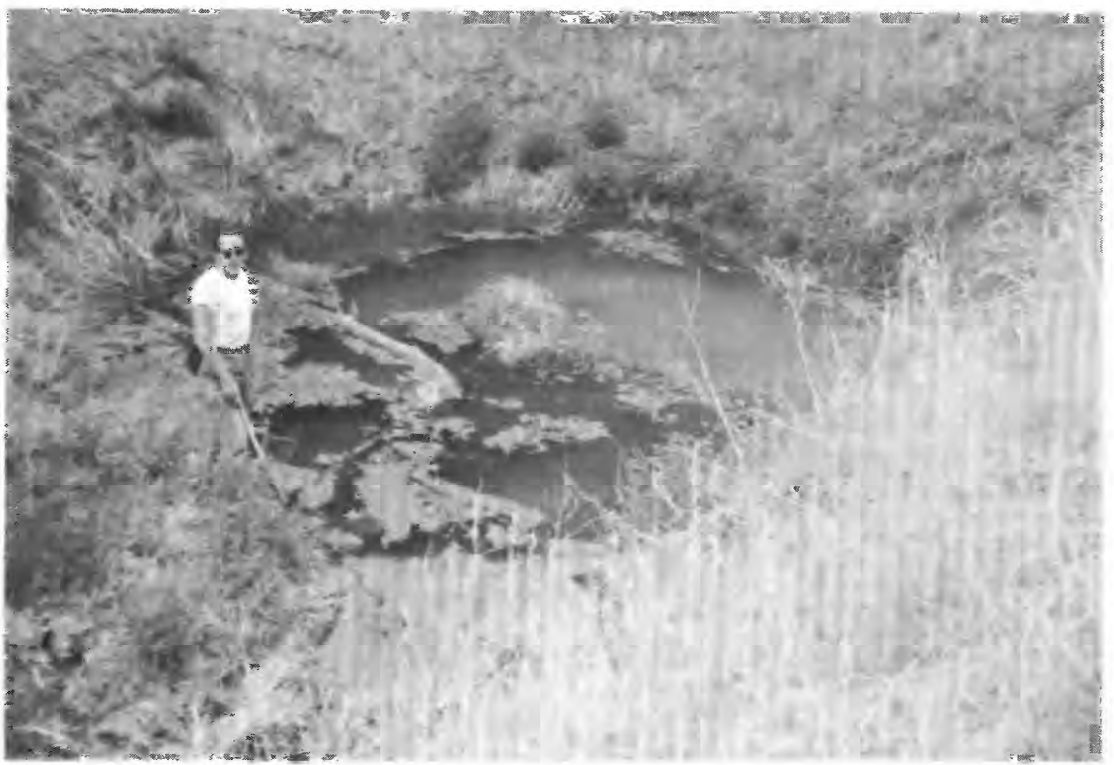

A. POOL AT BOTTOM OF CRATER

Casing is not visible and is presumed to be completely destroyed. Flow of $241 \mathrm{gpm}$ is discharged over land surface in lower right of photograph.

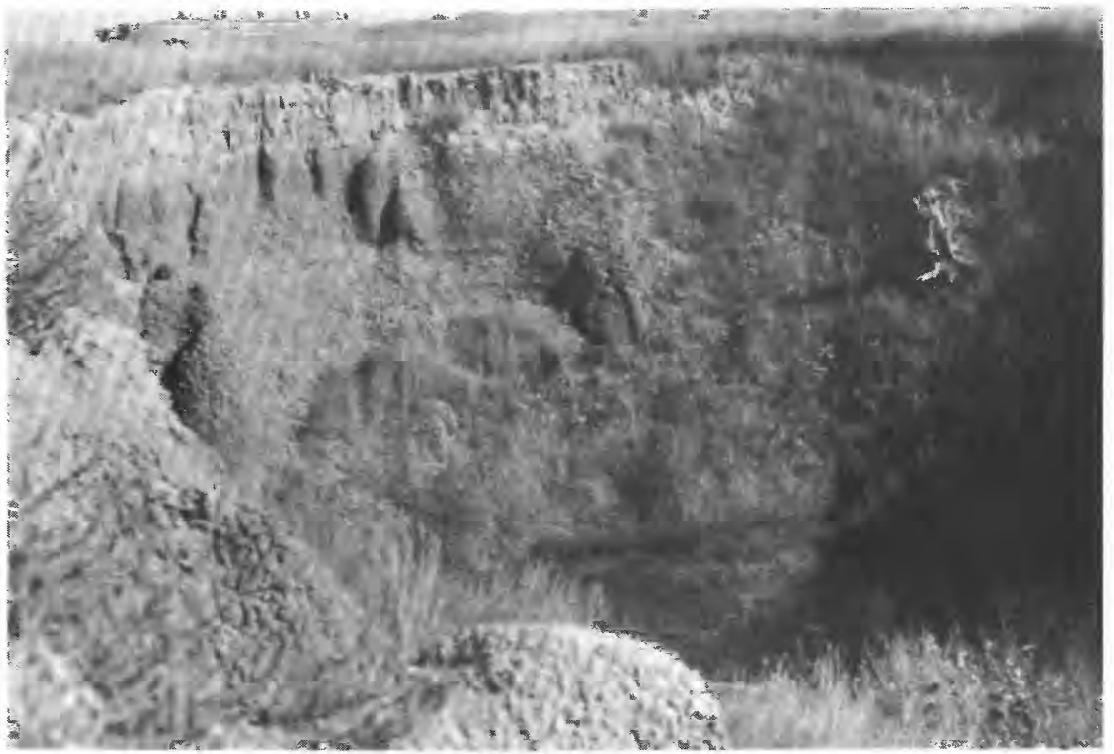

B. VIEW OF CRATER FROM NQRTHWEST RIM

Scale is indicated by car in background and by stump, which is about 4 feet in height. 
to be an important aquifer for flowing wells. The Fox Hills sandstone is a source of water for many pumped wells in northern South Dakota, however.

\section{CENOZOIC FORMATIONS-GLACIAL-DRIFT AQUIFERS OF QUATPRNARY AGE}

Water in buried glacial deposits of stratified sand and gravel east of the Missouri River generally occurs under artesian conditions, but flowing wells are obtained in only a few areas.

Along Choteau Creek, in Charles Mix County, glacial-drift aquifers consisting of stratified sand and gravel furnish water to both flowing and nonflowing wells. Probably the Niobrara formation, which (according to drillers' logs) is not present in this area, was removed by erosion prior to the deposition of sand and gravel. Flint's map (1955, pl. 7) of preglacial drainage shows a former valley along the site of present Choteau Creek.

Water from the sand and gravel deposits underlying the valley of Choteau Creek is under low artesian pressure, is cooler than water from the deeper Dakota sandstone, and, although reported to be somewhat mineralized, has a more agreeable taste than water from the Dakota. These glacial-drift aquifers constitute a potential source of water for irrigation, but quality-of-water studies are necessary to determine if the water is suitable for that purpose.

\section{RECHARGE TO ARTESIAN AQUIFERS IN SOUTH DAKOTA}

The piezometric surfaces of the various Paleozoic and Mesozoic artesian aquifers in South Dakota slope away from the Black Hills, indicating that this area is a source of recharge. Streams flowing outward from the Black Hills are reported to lose large amounts of water where they pass over cavernous Paleozoic limestone. This stream loss indicates that water is moving underground from the Black Hills to recharge artesian aquifers; however, when viewed on a regional scale (Darton, 1905, p. 192), the piezometric surface of the Dakota sandstone near the Black Hills is seen to have the shape of a mound beneath which water moves outward in all directions, superimposed on the generally eastward sloping piezometric surface. Thus, recharge to the Dakota sandstone occurs along the flanks of the Rocky Mountains, as well as around the Black Hills.

The Codell sandstone member of the Carlile shale, the Niobrara formation, and the Fox Hills sandstone probably are recharged more locally than is the Dakota sandstone. For example, at the Fort Randall dam in south-central South Dakota, the Niobrara formation occurs immediately below the Missouri River valley floor and is 
underlain by the Codell sandstone member. Water seeping into and through fractures in the Niobrara probably recharges both these aquifers in this area. Possibly the Codell is recharged in a similar manner at other locations in the Missouri River valley.

The Fox Hills sandstone probably receives small amounts of recharge in its outcrop areas in Butte, Meade, and Ziebach Counties.

Because little water appears to enter the Fall River formation at its outcrop in the Black Hills, except from precipitation, it becomes apparent that the water in the principal artesian aquifer must have moved into the aquifer by some method other than direct recharge at the outcrop. The strata immediately underlying the aquifer are relatively impermeable and act as a barrier to the upward movement of water from deeper aquifers that have greater artesian pressure than the Dakota. However, where there is a considerable difference in pressure between two aquifers, very slow movement of water can occur through even relatively impermeable confining material to the aquifer having the lower pressure. The principal artesian aquifer may receive a part of its recharge by such a method.

Water may also enter an aquifer from a deeper aquifer where both are penetrated by wells and neither flow is cased off. This situation may occur also if the casing of a well passing through two or more aquifers is sufficiently corroded to permit leakage. The amount of water from deeper aquifers entering the Dakota in this manner is not known; however, in areas having a large number of uncontrolled wells that tap deeper aquifers in addition to the Dakota, the amount may be large. Cratering or caving of the wells at the surface would increase the amount of water transferred from one aquifer to another.

\section{DISCHARGE FROM ARTESIAN AQUIFERS IN SOUTH DAKOTA}

Flowing wells throughout the State discharge a tremendous quantity of water. Many of the flowing wells inventoried in the valley of the Missouri River in Bon Homme, Brule, Buffalo, Charles Mix, Gregory, Lyman, and Yankton Counties are classed as uncontrolled wells. The flow from 46 of these wells is more than $16 \mathrm{mgd}$, most of it unused. For comparison, the total flow from 3,054 wells known or presumed to tap the Dakota sandstone in 3,500 square miles of the James River valley area is approximately $16 \mathrm{mgd}$ (U.S. Geol. Survey unpublished records).

The many pumped wells that penetrate artesian aquifers yield relatively insignificant amounts of water.

Water is also discharged from artesian aquifers by flow from artesian springs. In the vicinity of the Black Hills artesian springs occur 
in the Hot Springs area and in an area northwest of Spearfish, S. Dak. Near the city of Hot Springs the water issues from the Minnekahta limestone and northwest of Spearfish, it flows from the Spearfish formation. However, it is not definitely known whether the water originates in these formations or in some deeper aquifer. No artesian springs are positively identified as issuing from the Fall River and Lakota formations in the Black Hills, but Spring Creek in T. 6 N., R. 5 E., is reported to be fed by natural leakage from the Dakota sandstone, which crops out along the stream in this area.

Several springs in the valley of the Missouri River are believed to yield water from the Dakota sandstone. This belief is based on the similarities in temperature and chemical quality of water from the springs and water from wells tapping the Dakota sandstone in this area. Barkley (1952, p. 39) reported analyses of three such springs. It is possible that these springs may be old uncontrolled artesian wells. However, reports on the regularity of their flow and on the character of the springs themselves, indicate that they probably are natural springs. The most likely reason for the occurrence of springs in the valley is that the overburden above the Dakota sandstone is thinner than in the adjacent plain, thus permitting easier escape for water confined in that formation.

\section{SUMMARY OF DISCHARGE, BY COUNTIES}

\section{SOUTH DAKOTA}

A total of 38 counties were visited during the present study. The yields of selected wells inventoried in each county are briefly discussed on the following pages.

The location of wells having flows in excess of $20 \mathrm{gpm}$ is shown on plate 1. Flows of these wells were measured by a portable aluminum Parshall flume. The flume was placed in the discharge channel 5 to 30 feet from the wellhead and the height of the water column passing through the flume was measured in a stilling well at the side of the flume. (See plate 2.) Discharge was calculated from the measurement in the stilling well.

Aurora County.-Well 104-63-28bbbbl, a former oil-test hole, flows $31 \mathrm{gpm}$. Only one other well was inventoried in the county, and this flow is not known to be representative of other flows in the county.

Beadle County.-Wells in Beadle County generally have small flows, ranging from less than 1 to $15 \mathrm{gpm}$. Well 113-61-28adcal, however, flows $209 \mathrm{gpm}$ - the largest known rate of flow in the James River valley area. 
Bon Homme County.-One large uncontrolled well (93-6023ddcd1) in the city of Springfield flows approximately $500 \mathrm{gpm}$. The flow may affect the head of the city wells, which are believed to tap the same aquifer.

Near wells 92-60-3ba1 and 92-60-3ba2, west of Springfield, iron casings are reported to deteriorate in about 3 years. This deterioration is due to the amount of flow, pressure, and chemical quality of the water. The "tee" shown in plate $4, A$ illustrates the corrosive properties of some of the water from the Dakota sandstone.

In the same area, water from undeveloped springs at the base of a bluff of marly chalk of the Niobrara formation has a temperature of $65^{\circ} \mathrm{F}$. and a specific conductance of 1,980 micromhos, about the same conductance as water from artesian wells that are at a higher elevation and tap the Dakota sandstone. These springs are probably fed by artesian leakage from old wells in the same area. A spring known to yield water from the Niobrara formation about a mile south has a temperature of $55^{\circ} \mathrm{F}$. and a specific conductance of 1,820 micromhos. Although artesian leakage into other aquifers has been reported at some places elsewhere in the State, and undoubtedly occurs unseen and unknown at many others, this is the only known locality where it is reasonably certain such leakage occurs and is visible at the surface.

Brule County.-Locations of artesian wells inventoried in Brule County are shown on plate 2. In the valley of the Missouri River, nine uncontrolled wells flowing 5 to $2,570 \mathrm{gpm}$ yield a total of about $3,400 \mathrm{gpm}$. Well 103-70-17ccbc1, which flows into Red Lake, yields $1,250 \mathrm{gpm}$. Of 108 flowing wells, less than 9 percent-that is, the 9 uncontrolled wells and the well feeding Red Lake-yield 87 percent, or about $4,650 \mathrm{gpm}$, of the total discharge from all flowing wells in the county. Of the 563 wells in the county that probably tap the Dakota sandstone, 108, or 19 percent, are flowing wells. The remaining 455 nonflowing wells, either are pumped or are abandoned. The total flow of the 108 flowing wells is about $5,370 \mathrm{gpm}$. Well $104-71-10 \mathrm{abd} 1$ at the time of the inventory was reported to be normally shut off and is not included in the 108 . If every one of the 455 known nonflowing wells in the Dakota sandstone in the county were pumped continuously at the rate of $1 \mathrm{gpm}$ (1,440 gallons per day) they would yield 655,000 gallons per day, the same amount of water that flows unused from 3 of the uncontrolled wells (102-71-6babb1, 102-72-2aadc1, and 107-72-2cbda1). However, this amount is less than a fifth as much as the 3,700,000 gpd of water that flows from abandoned wells (104-71-15abac1 and 104-71-15abac2) at the former electric plant at Chamberlain, S. Dak. 
Much of Brule County depends on the Dakota sandstone for the only continuously available supply of ground water. It is evident that large unused flows in this and adjacent counties are depleting this supply and are lowering the water levels in all wells.

Buffalo County.-Only the Fort Thompson area in Buffalo County has been inventoried. An unrestrained well (107-72-22dbbc1) flows $183 \mathrm{gpm}$ and a cratered well (107-72-23bdab1) flows $94 \mathrm{gpm}$.

Butte County.-Well A-9-3-27addb1, a former oil-test hole, flows unused and unrestrained at an approximate rate of $2,000 \mathrm{gpm}$ (pl. $4, B)$. Because this well taps deep aquifers that may be important for future supplies of water, bringing it under control should be worthwhile. Other wells inventoried in Butte County tap the Fall River or the Lakota formation. Well A-8-3-25ddcb1, used for irrigation, has no control mechanism and flows continuously.

Charles Mix County.-In the valley of the Missouri River in Charles Mix County, the flow from many wells is unused. In this area 13 uncontrolled or unrestrained wells flowing 25 to $1,500 \mathrm{gpm}$ have a total discharge of about $4,200 \mathrm{gpm}$. Cratered well 100-71-26dbbb1 furnishes a good example of wasteful flow. (See pl. 5.) The water rises at an undetermined point in a crater 80 feet in diameter and flows down a gully to the nearby Missouri River. Occasionally a few cattle drink from the approximately 2 million gallons of water per day wasted from this well The well is estimated to have flowed more than 50 billion gallons of water since it was drilled in 1895; this volume is slightly greater than the normal maximum storage of Angostura Reservoir near Hot Springs, S. Dak.

A map showing the location of artesian wells inventoried in Charles Mix and Gregory Counties is shown on plate 3. Well 98-68-31dbbb1 is shown on plate 6. Well 95-65-35addd1 $(\mathrm{pl} .7, A)$ is an example of a well having an unrestrained flow.

Codington County.-Well 116-52-2cbbb1 is the only well reported to tap a deep artesian aquifer in Codington County. This well does not flow, and it is not used because of the poor chemical quality of the water.

Custer County.-The main area of artesian flow in Custer County is in the valley of the Cheyenne River. Flows from individual wells in this area are generally moderate (less than $40 \mathrm{gpm}$ ).

Dewey County.-The municipal well in the city of Eagle Butte obtains a reported flow of $115 \mathrm{gpm}$ from the Pahasapa limestone. Flowing wells that tap the Dakota sandstone are reported to be along the Moreau River.

Edmunds and Faulk Counties.-No unused wells having large flows are reported for Edmunds County or Faulk County. 
Fall River County.--In Fall River County the main area of flowing wells is in the valley of the Cheyenne River. These wells tap sandstones in the Fall River and Lakota formations. Flows are nominal but usually exceed minimum needs.

In Edgemont, flowing wells tap the Minnelusa sandstone, and at the Black Hills Ordnance Depot at Igloo, wells have obtained flows from the Pahasapa and Englewood limestones.

Gregory County.-There are several unused flowing wells in the valley of Whetstone Creek. Uncontrolled well 97-68-29abc1 (pl. 7, $B)$, with a flow of about $600 \mathrm{gpm}$, and cratered well 97-69-25bcda1, with a flow of $97 \mathrm{gpm}$, are the largest known flowing wells along Whetstone Creek. Well 97-68-28dd1, now inundated by Fort Randall reservoir, flowed about $300 \mathrm{gpm}$ in 1949. Flows in other parts of the county generally are greater than needed. However, in most of the county, wells tapping the Dakota sandstone must be pumped.

Haakon County.-Water from flowing wells in Haakon County generally is warmer than $100^{\circ} \mathrm{F}$. and commonly contains combustible gas. Most flowing wells in the county discharge more water than is used. An unrestrained flow of more than $30 \mathrm{gpm}$ from one well is permitted so as to prevent the accumulation of algae in the adjacent stock tank.

Hand County.-No flowing wells of large yield are reported for Hand County.

Harding County.-Few wells in Harding County tap deep artesian aquifers. Well A-17-4-16bdc1 flows an estimated $1 \mathrm{gpm}$.

Hughes County.-The two artesian wells at the State Capitol have the largest flows of wells visited in Hughes County. Water from the Dakota sandstone in this part of the State generally contains gas and is not potable.

Hutchinson County.-The main area of artesian flow in Hutchinson County is in the valley of the James River. Well 97-58-10abbd1 flows $116 \mathrm{gpm}$ and well 97-59-2adbd1 flows $93 \mathrm{gpm}$, the largest flows observed in the county.

Hyde County.-The altitude of the land surface of most of Hyde County is too great to permit water to flow from wells in the Dakota sandstone. No wells of large flow are reported for the county.

Jackson and Jones County.-No wells of large flow are reported for Jackson County or Jones County.

Lawrence County.-Irrigation wells penetrating the Minnekahta limestone and the Spearfish formation can produce large flows but are usually shut off when water is not needed. However, wells A-7-1-21dca1 and A-7-2-19cca1 flow $43 \mathrm{gpm}$ and more than $300 \mathrm{gpm}$, respectively, throughout the year. 
Lyman County.-The valley of the White River near its junction with the Missouri River in Lyman County, is an area of extensive waste of water from flowing wells. Thirteen wells in this area flow a total of about $1,700 \mathrm{gpm}$. Almost all the water is unused, and the casings of most of the wells are in poor condition. Eighty-six percent of the water $(1,450 \mathrm{gpm})$ comes from only six wells. Two of these wells are shown on plates 8 and 9 .

McPherson County.-No large-capacity flowing wells are known to pentrate the Dakota sandstone in McPherson County; however, wells drilled to deeper artesian aquifers should be capable of yielding large quantities of water under high pressure.

Meade County.-The important areas of artesian flow in Meade County are in the valley of the Cheyenne River and along the east side of the Black Hills near the outcrop area of most of the formations previously discussed. Flows are small and moderate except in the eastern part of the county where some wells of large flow are reported.

Mellette County.-The Dakota sandstone is penetrated by five wells in Mellette County. At the time of inventory three of these wells were flowing much more water than was used.

Miner County.-No wells having large unused flows are reported for Miner County.

Pennington County.-Few wells tap the Dakota sandstone in Pennington County. Near Rapid City older strata are reported to supply artesian water.

Sanborn County.-Few wells having large unused flows are reported to tap the Dakota sandstone in Sanborn County. In some areas shallow wells about 100 feet deep tap glacial-drift aquifers that yield water by natural flow. Throughout the county many abandoned wells flow small amounts of unused water.

Spink County.-No wells of large flow are reported for Spink County.

Stanley County.-Because of its high salinity and dissolved gases, most of the water from the Dakota sandstone in Stanley County is unfit for human consumption. However, there are several wells of large flow in the valleys of the Bad River and its tributaries and in the valley of the Missouri River.

The Pahasapa and Minnelusa formations, of Paleozoic age, and apparently the Sundance formation, of Mesozoic age, underlie most of Stanley County and in the future may prove to be important artesion aquifers. These deeper aquifers have been reached only by oiltest holes to date (1958). 
Sully County.-Few wells of large flow tap the Dakota sandstone in Sully County. However, wells penetrating deeper aquifers yield large quantities of water under high artesian pressure. Well 114-772-dca1, at Onida, which is reported to tap the Sundance formation, is classed as uncontrolled, owing to a broken casing and high artesian pressure.

Tripp County.-Few wells of large flow are reported to be in Tripp County. However, several wells in the valleys of streams tributary to the White River have flows in excess of $20 \mathrm{gpm}$.

Union County.-Artesian pressure in the Dakota sandstone apparently is not great enough to force water to flow to the land surface in Union County.

Yankton County.-About $560 \mathrm{gpm}$ of water is wasted from three of five unused wells in the city of Yankton. In other parts of the county, flows are nominal, but a few wells in the James River valley flow more water than is used. Three unused wells at the abandoned cement plant near Yankton discharge about $115 \mathrm{gpm}$.

Ziebach County.-At the time of the inventory, well A-9-19-6-a1 had a large but unmeasured flow of unused water.

\section{WYOMING}

Crook County.-Well 53-60-28cc3 is believed to be the best available well for measuring the water level in the Minnekahta limestone. Several artesian irrigation wells tap the Minnekahta in nearby Lawrence County, S. Dak.

Weston County.-Wells 41-60-7dad1 and 41-60-7dbbb1 have a combined flow of about $500 \mathrm{gpm}$, most of which is unused.

\section{CONCLUSIONS}

Unused flowing wells in South Dakota are discharging large quantities of water that is reducing the artesian pressure and decreasing the area in which other flowing wells can be obtained. Although these uncontrolled wells of large discharge are the major cause of waste of water, any well whose flow is only slightly in excess of the need is contributing to the depletion of a vital natural resource.

The decrease in artesian pressure in aquifers penetrated by flowing wells will bring about a corresponding decline in the water levels of nonflowing wells that tap the same aquifer at higher elevations. As the water levels in these wells decline, pumping costs increase. Decline of the water level may necessitate the purchase of new pumping equipment or even the drilling of a new well.

The piezometric surfaces of the major artesian aquifers in South Dakota have been declining since the first wells were drilled; the 
earliest, in 1881. Contiinued drilling of wells that tap artesian aquifers has lowered the piezometric surface until in many areas there are now no flowing wells. This is the ultimate result of the waste of water in any artesian basin. Continued discharge of water from unused flowing wells is hastening the day when all artesian wells in the State will cease to flow.

\section{SELECTED BIBLIOGRAPHY}

Baker, C. L., 1947, Deep borings of western South Dakota: South Dakota Geol. Survey Rept. Inv. 57, 112 p.

1948, Additional well borings in South Dakota (Supplement to Rept. Inv. 57) : South Dakota Geol. Survey Rept. Inv. 61, 40p.

1951, Well borings in South Dakota, 1948-50: South Dakota Geol. Survey Rept. Inv. 67, $67 \mathrm{p}$.

Barkley, R. C., 1952, Artesian conditions in southeastern South Dakota: South Dakota Geol. Survey Rept. Inv. 71, 71 p.

1953, Artesian conditions in area surrounding the Sioux quartzite ridge: South Dakota Geol. Survey Rept. Inv. 72, 68 p.

Darton, N. H., 1896, Preliminary report on artesian waters of a portion of the Dakotas: U.S. Geol. Survey 17th Ann. Rept., pt. 2, p. 603-694.

1905, Preliminary report on the geology and underground water resources of the central Great Plains : U.S. Geol. Survey Prof. Paper 32, $433 \mathrm{p}$. 1909, Geology and underground waters of South Dakota: U.S. Geol. Survey Water-Supply Paper 227, $156 \mathrm{p}$.

1918, Artesian waters in the vicinity of the Black Hills, S. Dak. : U.S. Geol. Survey Water-Supply Paper 428, 64 p.

Erickson, H D., 1954, Artesian conditions in east central South Dakota: South Dakota Geol. Survey Rept. Inv. 74, $116 \mathrm{p}$.

1955, Artesian conditions in northeastern South Dakota: South Dakota Geol. Survey Rept. Inv. 77, 39 p.

Flint, R. F., 1955, Pleistocene geology of eastern South Dakota: U.S. Geol. Survey Prof. Paper 262, 158 p.

Gries, J. P., 1954, Cretaceous rocks of Williston basin : Am. Assoc. Petroleum Geologists Bull., v. 38, no. 4, p. 443-453.

Loucks, D. W., 1940, Seventeenth report of the State Engineer [South Dakota] for the period July 1, 1938, to June 30, 1940:112 p., Office of State Engineer, Pierre, S. Dak.

1942, Eighteenth report of the State Engineer [South Dakota] for the period July 1, 1940, to June 30, 1942: 105 p., Office of State Engineer, Pierre, S. Dak.

- 1944, Nineteenth report of the State Engineer [South Dakota] for the period July 1, 1942, to June 30, 1944: 69 p., Office of State Engineer, Pierre, S. Dak.

1946, Twentieth report of the State Engineer [South Dakota] for the period July 1, 1944, to June 30, 1946: 95 p., Office of State Engineer, Pierre, S. Dak.

1948, Twenty-first report of the State Engineer [South Dakota] for the period July- 1, 1946, to June 30, 1948: 116 p., Office of State Engineer, Pierre, S. Dak. 
Loucks, D. W., 1952, Twenty-third report of the State Engineer [South Dakota] for the period July 1, 1950, to June 30, $1952: 306$ p., Office of State Engineer, Pierre, S. Dak.

Meinzer, O. E., 1923, The occurence of ground water in the United States, with a discussion of principles: U.S. Geol. Survey Water-Supply Paper 489, 321 p. Rothrock, E. P., 1934, Water supplies at Fort Thompson, S.Dak.: South Dakota Geol. Survey Rept. Inv. 18, 10 p.

1936, Logs of some deep wells in western South Dakota: South Dakota Geol. Survey Rept. Inv. 4; rev. ed., 1946, 108 p.

1943, A geology of South Dakota: South Dakota Geol. Survey Bull. 13, $88 \mathrm{p}$.

Rothrock, E. P., and Robinson, T. W., Jr., 1936, Artesian conditions in west central South Dakota : South Dakota Geol. Survey Rept. Inv. 26, 2d ed., 1938, $93 \mathrm{p}$.

South Dakota State College Extension Service, 1940, Rural water supplies in South Dakota: Spec. Ext. Circ. 47 (41 separate pamphlets, by counties, 15-27 p. each).

South Dakota State Department of Health, 1956, South Dakota public water supply data : 19 p., Pierre, S. Dak.

South Dakota State Planning Board, 1937, Artesian well flow in South Dakota: 138 p.. Central Office, Brookings, S. Dak.

Todd, J. E., 1895, A preliminary report on the geoiogy of South Dakota: South Dakota Geol. Survey Bull. 1, 172 p.

1896, Geology of South Dakota: South Dakota Geol. Survey Bull. 2, 139 p. 
RECORDS OF SELECTED ARTESIAN WELLS 


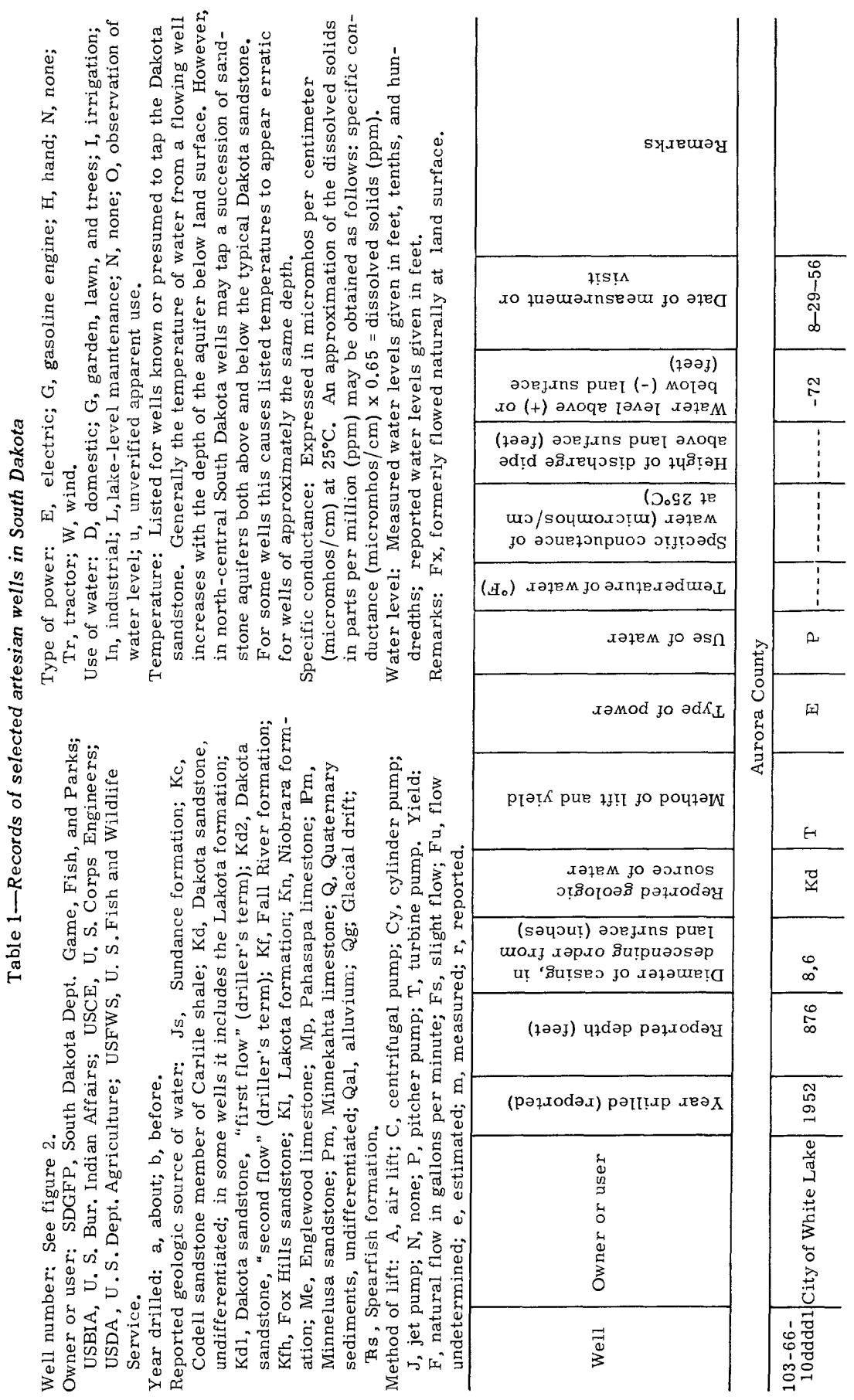




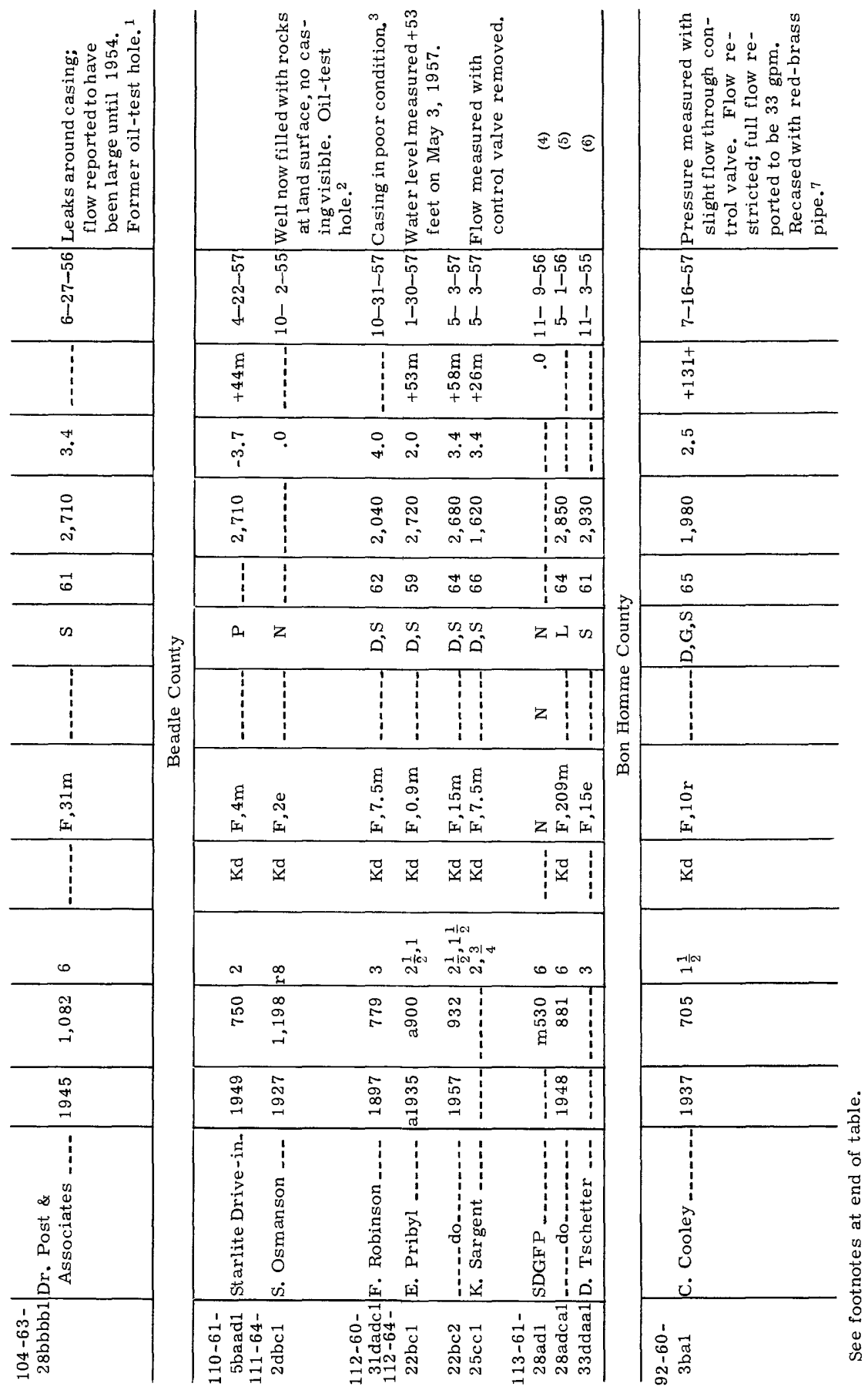




\begin{tabular}{|c|c|c|c|}
\hline sy.дешәy & 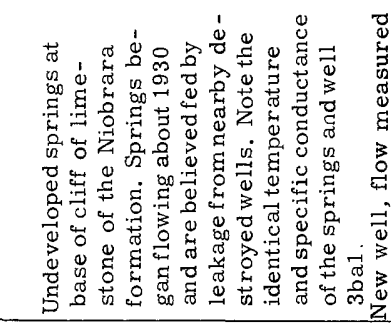 & 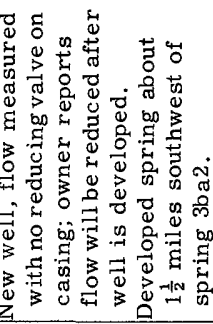 & 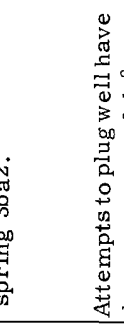 \\
\hline 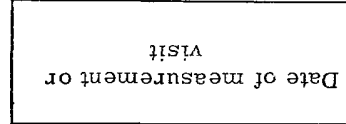 & $\begin{array}{ll}2 & 5 \\
0 & 5 \\
0 & 0 \\
1 & 1 \\
1 & 1\end{array}$ & $\begin{array}{ll}1 & \\
5 & 5 \\
1 & 1 \\
0 & 1 \\
1 & 1 \\
1 & 1\end{array}$ & $\begin{array}{l}0 \\
10 \\
1 \\
\infty \\
1 \\
1\end{array}$ \\
\hline 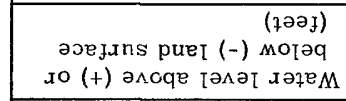 & $\begin{array}{c}3 \\
\vdots \\
\vdots \\
\vdots\end{array}$ & $\begin{array}{l}3 \\
\vdots \\
\vdots \\
\end{array}$ & $\vdots$ \\
\hline 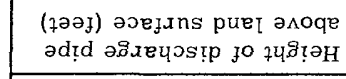 & 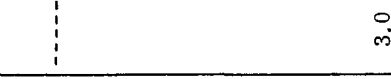 & 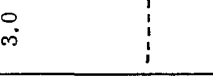 & $\begin{array}{l}\infty \\
\dot{i}\end{array}$ \\
\hline 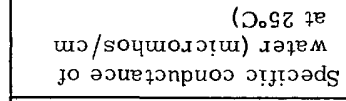 & $\begin{array}{l}8 \\
\infty \\
\infty \\
- \\
-1\end{array}$ & 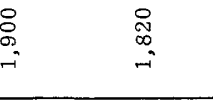 & $\begin{array}{l}\text { H } \\
\text { N } \\
\text { N }\end{array}$ \\
\hline (но) ләұем јо әлпұеләdшә $L$ & $\stackrel{8}{0}$ & 㶽 & 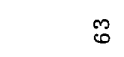 \\
\hline גәтем јо әs & $\infty$ & n & $z$ \\
\hline IəMOd jo $\operatorname{\partial d} K_{\mathrm{I}}$ & 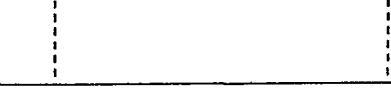 & 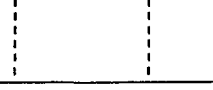 & 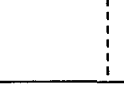 \\
\hline 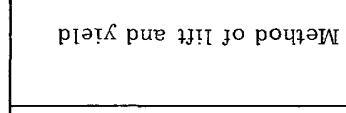 & 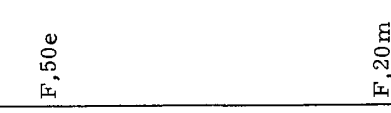 & $\stackrel{\infty}{\llcorner}$ & 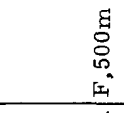 \\
\hline 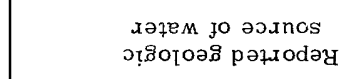 & : & 部 & 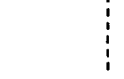 \\
\hline 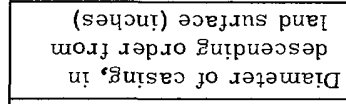 & $\begin{array}{c}\vdots \\
\vdots \\
\vdots \\
\vdots\end{array}$ & 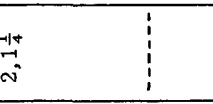 & $\begin{array}{l}0 \\
\infty\end{array}$ \\
\hline (ұәәј) чұาdәр рәұлодәч & 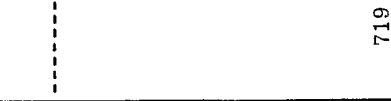 & 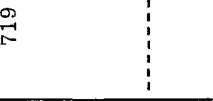 & 용 \\
\hline (рәұлоdəл) рәІт!ир леә & 点 & $\vdots$ & ஓ \\
\hline 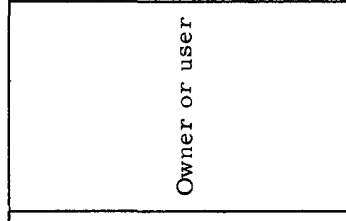 & $\begin{array}{l}1 \\
1 \\
\vdots \\
0 \\
0 \\
0 \\
0 \\
0 \\
0\end{array}$ & 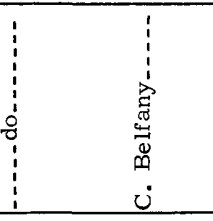 & 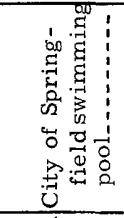 \\
\hline$\vec{\omega}$ & $\begin{array}{l}1 \\
\dot{0} \\
0 \\
1 \\
\text { N } \\
\infty\end{array}$ & $\begin{array}{l}7 \\
\overline{8} \\
\overline{8}\end{array}$ & 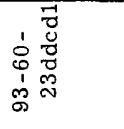 \\
\hline
\end{tabular}




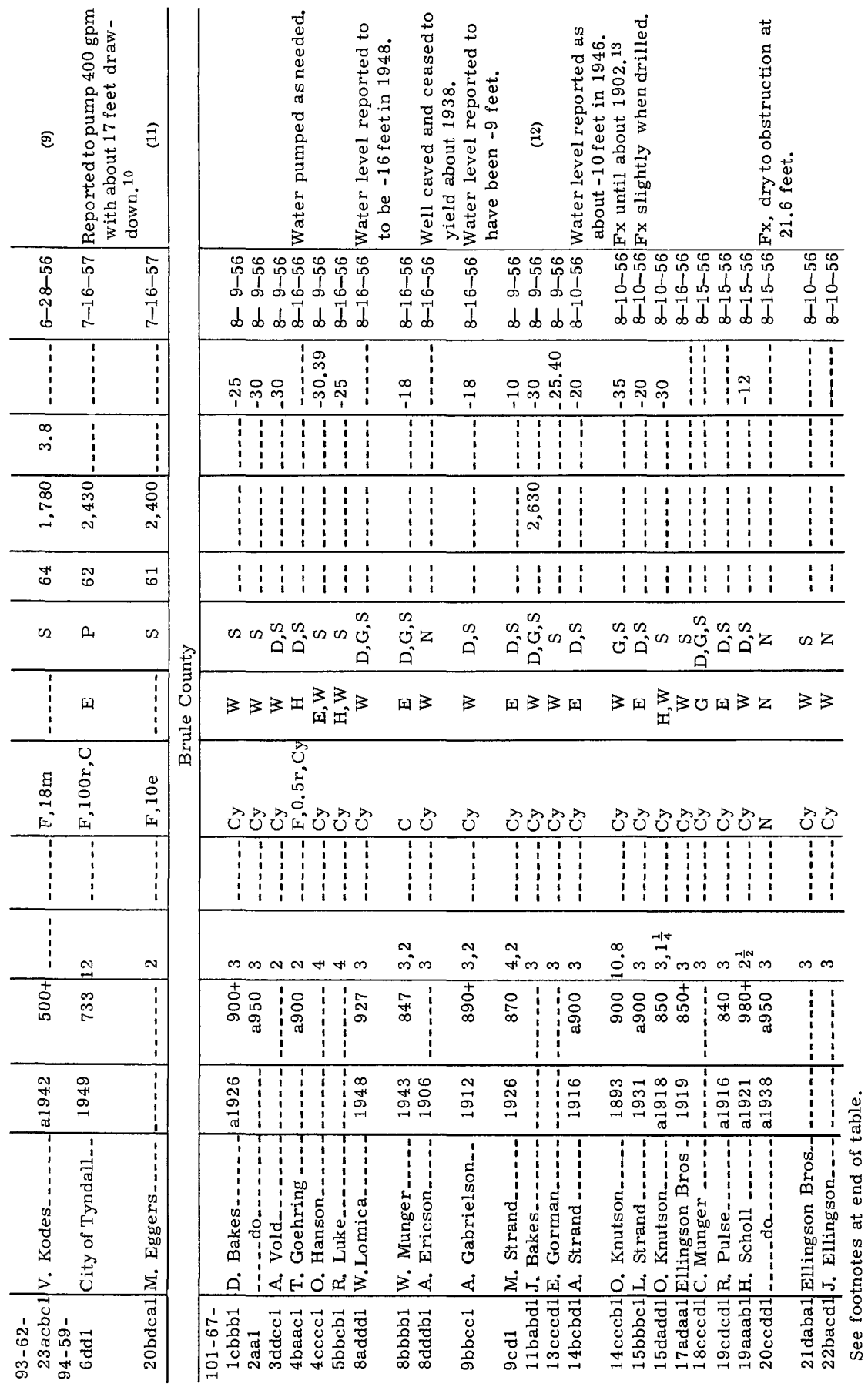




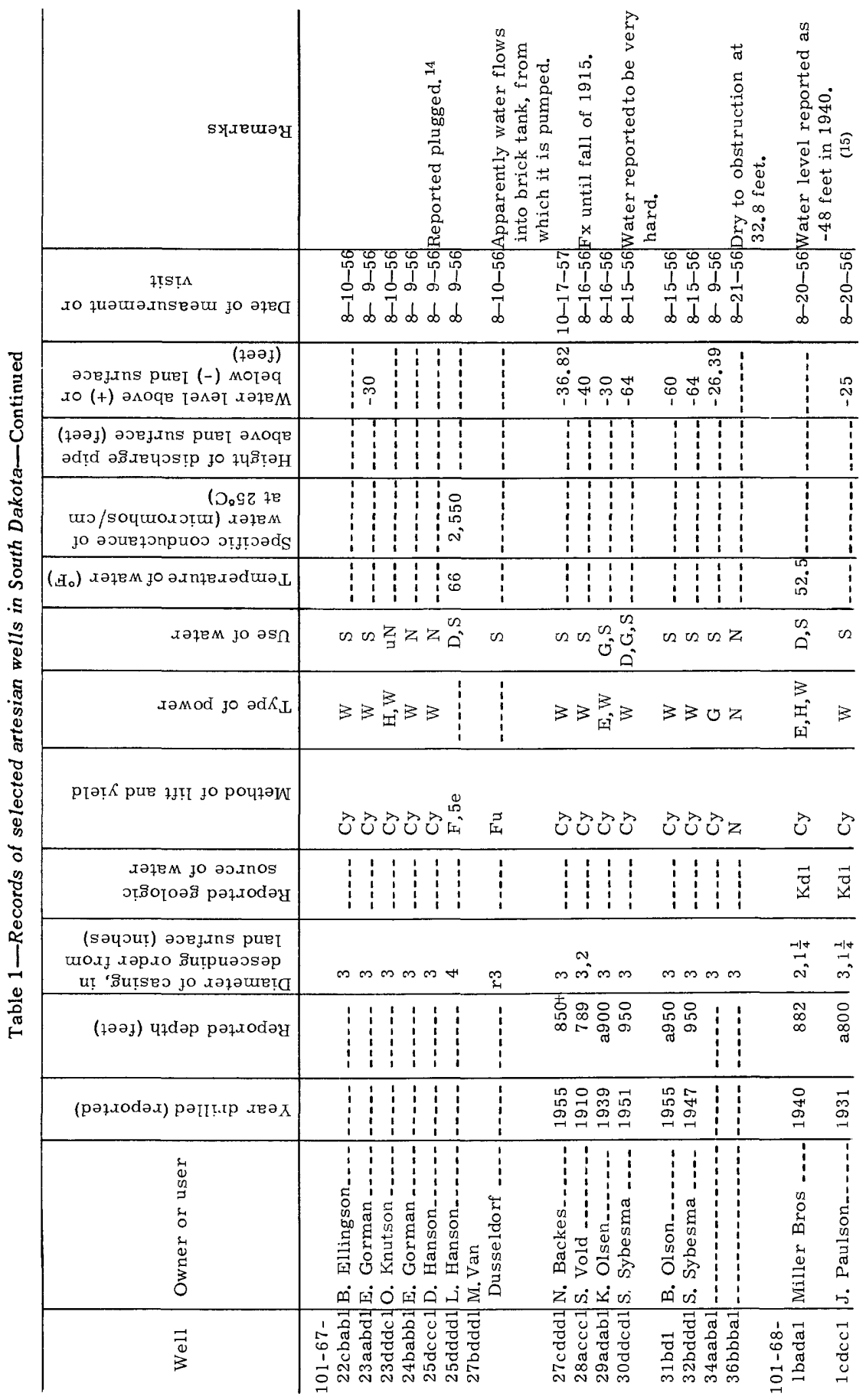




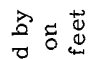

苟出

这出

这宗

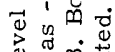

ये

\&

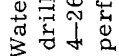

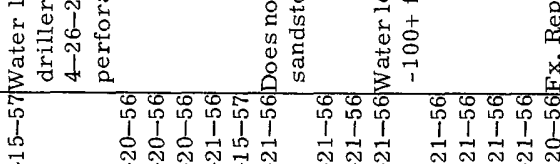
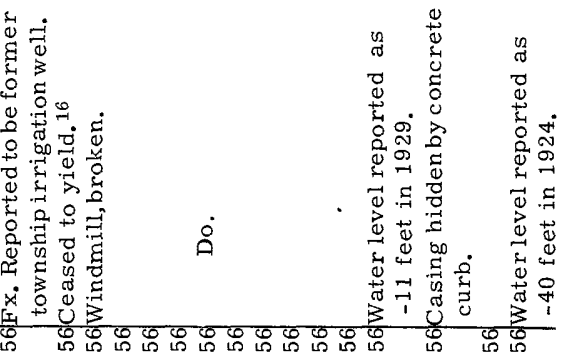

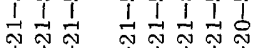

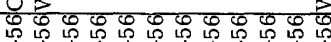

$\dot{8}$

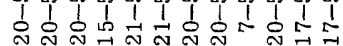

$1 \quad 11$

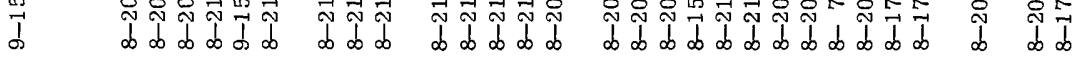

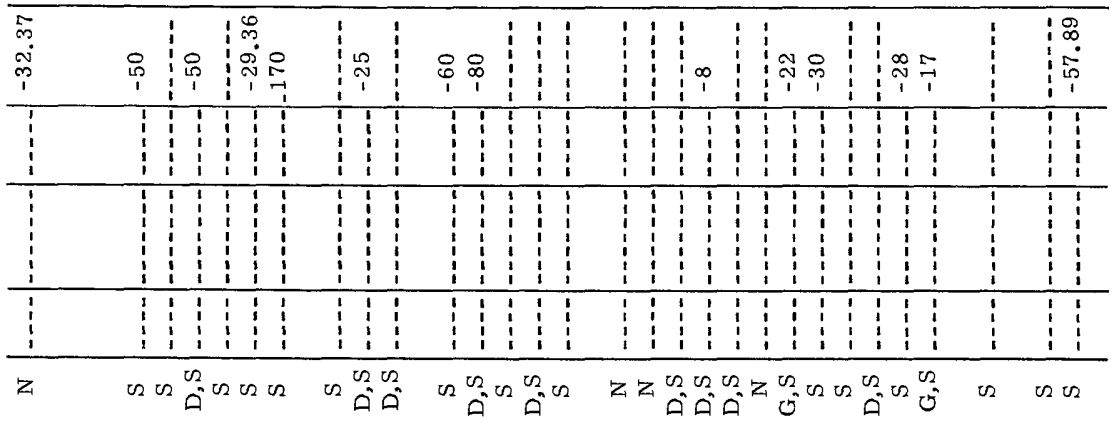

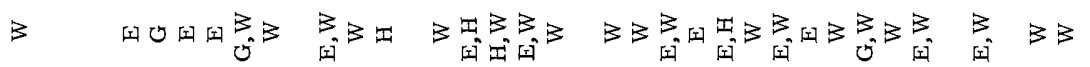

ठ

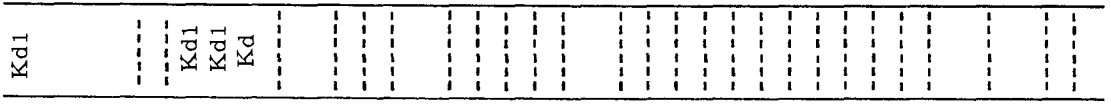

(1)

$\dddot{2}$

2

$553972 \mathrm{O}-61-3$ 


\begin{tabular}{|c|c|c|c|c|c|c|}
\hline Syдеuәy & 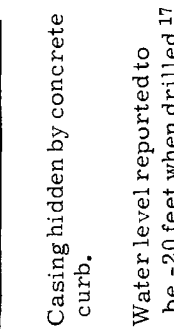 & 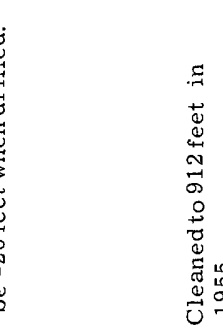 & 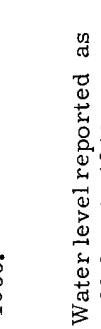 & & 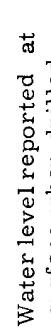 & 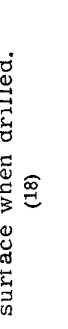 \\
\hline 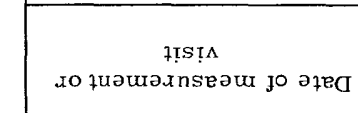 & 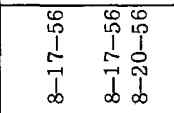 & 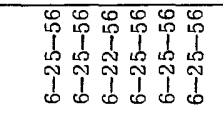 & $\begin{array}{lll}0 & 0 & 0 \\
0 & 0 & 0 \\
1 & 1 & 1 \\
0 & 0 & 0 \\
0 & 0 & 0 \\
1 & 1 & 1 \\
0 & 0 & 0\end{array}$ & $\begin{array}{l}0 \\
10 \\
1 \\
\omega \\
1 \\
1 \\
0\end{array}$ & $\begin{array}{l}5 \\
10 \\
1 \\
1 \\
0\end{array}$ & 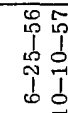 \\
\hline 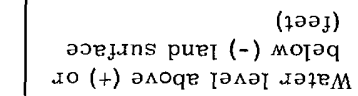 & 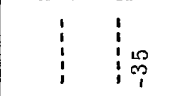 & $\begin{array}{llll} & & & \\
1 & 0 & 0\end{array}$ & & $\vdots$ & $\begin{array}{l}9 \\
m \\
\infty \\
i \\
1\end{array}$ & 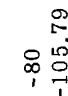 \\
\hline 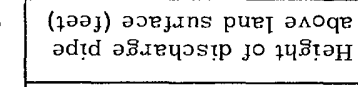 & $i$ & 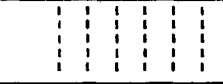 & $\begin{array}{l:c} & \vdots \\
\vdots & \vdots \\
1 & \vdots \\
\end{array}$ & $\vdots$ & 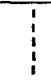 & \\
\hline 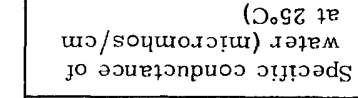 & 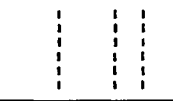 & 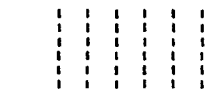 & 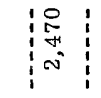 & $\vdots$ & $\vdots$ & 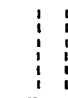 \\
\hline 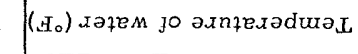 & $\begin{array}{l}1 \\
i\end{array}$ & 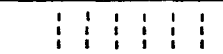 & $1 \stackrel{10}{10}$ & $i$ & 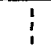 & $i$ \\
\hline xә7ем јо әs $\Omega$ & $\begin{array}{ll}0 & 0 \\
0 & 0\end{array}$ & $\cos \theta \cos ^{2} \cos \frac{\pi}{a}$ & $\begin{array}{l}n=2 \pi \\
00^{2}\end{array}$ & is & z & $\begin{array}{l}0,0 \\
00 \\
00 \\
00\end{array}$ \\
\hline גəMOd วо $ә \mathrm{~d} \kappa_{\mathrm{L}}$ & 3 回 3 & 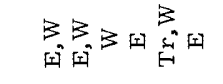 & 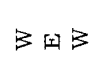 & 红 & z & 더 되 \\
\hline 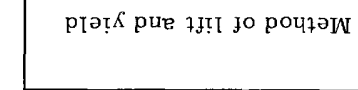 & $\overrightarrow{0} \overrightarrow{0}$ & 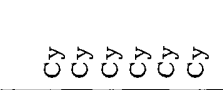 & $\overrightarrow{0} \theta \overrightarrow{0}$ & $\overrightarrow{0}$ & $z$ & $m$ \\
\hline 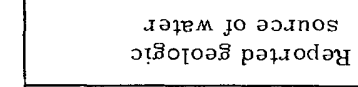 & 15 & 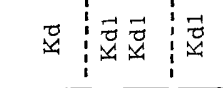 & & 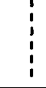 & 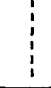 & $\begin{array}{l:c}1 \\
\vdots \\
\vdots\end{array}$ \\
\hline 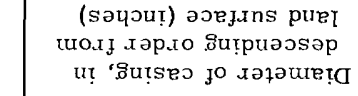 & $\infty$ & $\operatorname{manmms}$ & $m \infty m$ & $\stackrel{\text { Hi }}{N}$ & $\stackrel{-14}{m}$ & $\begin{array}{l}m^{-1 N} \\
N \\
m\end{array}$ \\
\hline (ұәәј) чұdәр рәұлодәч & $\mathfrak{l}_{\substack{\infty \\
\infty}}^{\infty}$ & 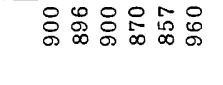 & $\begin{array}{l:l}\circ \\
\stackrel{\infty}{\circ} \\
\circ\end{array}$ & $\begin{array}{c}\infty \\
\stackrel{\infty}{0} \\
\infty\end{array}$ & $\begin{array}{l}\stackrel{\text { D }}{\mathrm{O}} \\
\stackrel{-}{i}\end{array}$ & $\begin{array}{l:l}0 & \\
- & \vdots \\
0 & \\
-i & 1\end{array}$ \\
\hline (рәұлоdәx) рәтцир $x$ вәX & 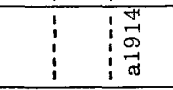 & 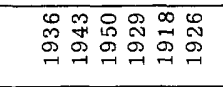 & \begin{tabular}{l:l}
\multirow{D}{*}{} & $\infty$ \\
Oे & $\stackrel{+}{\circ}$ \\
ते & -1
\end{tabular} & $\begin{array}{l}\infty \\
\stackrel{\infty}{\circ} \\
\rightarrow\end{array}$ & $\begin{array}{l}\text { m } \\
0 \\
0 \\
\text { तె }\end{array}$ & 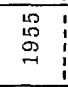 \\
\hline 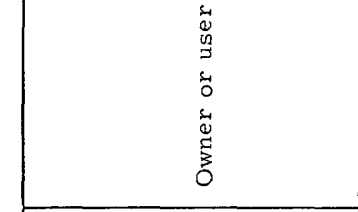 & 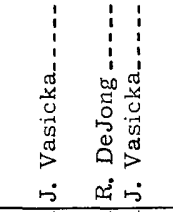 & 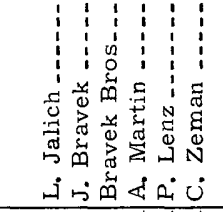 & 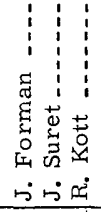 & 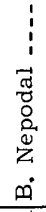 & $\begin{array}{c}1 \\
\vdots \\
\vdots \\
\vdots \\
\vdots \\
\vdots \\
\vdots\end{array}$ & 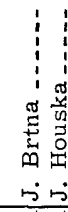 \\
\hline $\overrightarrow{\vec{\omega}}$ & 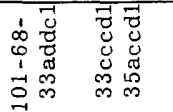 & 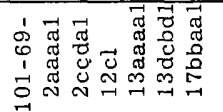 & 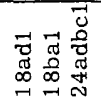 & 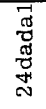 & 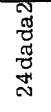 & 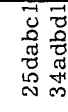 \\
\hline
\end{tabular}




\begin{tabular}{|c|c|c|c|c|c|c|}
\hline 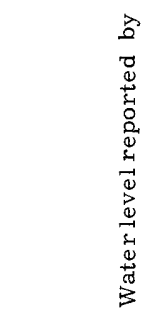 & 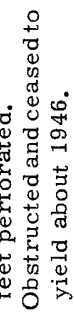 & 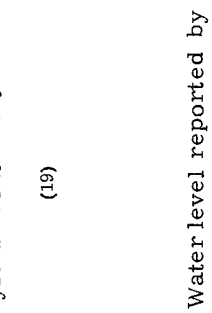 & क्षे & 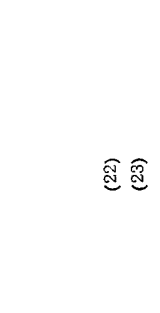 & 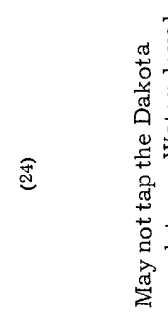 & \\
\hline $\begin{array}{llll}0 & 0 & 0 & 0 \\
0 & 0 & 0 \\
1 & 1 & 1 & 1 \\
1 & 1 & 1 \\
0 & 0 & 0 & 0 \\
0 & 0 & 0 & 0\end{array}$ & $\begin{array}{l}0 \\
0 \\
1 \\
1 \\
0 \\
0\end{array}$ & 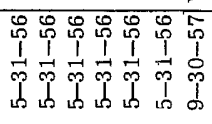 & $\begin{array}{lll}0 & 0 & 0 \\
0 & 0 & 0 \\
1 & 1 & 1 \\
1 & 1-1 & 1 \\
0 & 0 & 0 \\
0 & 1 & 1 \\
0 & 0 & 0\end{array}$ & 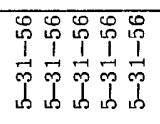 & $\begin{array}{lllll}0 & 0 & 0 & 0 \\
0 & 0 & 0 \\
0 & 0 & 0 & 0 \\
0 & 1 & 1 & 1 \\
0 & 0 & 1 & 1 \\
0 & 0 & 0 & 0 \\
0 & 1 & 1 & 1 & 1 \\
0 & 1 & 1 & 1\end{array}$ & $\begin{array}{l}0 \\
1 \\
1 \\
1 \\
1\end{array}$ \\
\hline$\left\{\begin{array}{lll}0 & + \\
8 & 0 & 0 \\
1 & 1 & 1 \\
1 & 1\end{array}\right.$ & 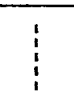 & 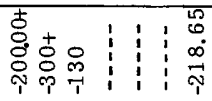 & & 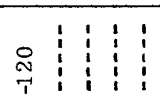 & 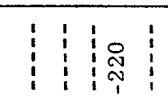 & i⿱ \\
\hline & & 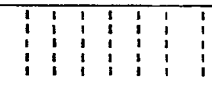 & & 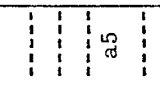 & 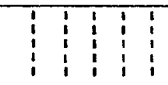 & 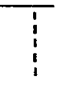 \\
\hline 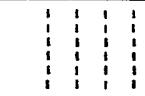 & 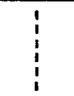 & 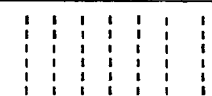 & 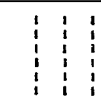 & 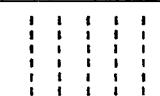 & 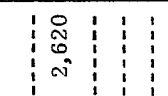 & \\
\hline $\begin{array}{l:lll} & 1 & 1 & \vdots\end{array}$ & i & \begin{tabular}{l|llllll} 
& & & 1 & 1 & 1 & 1 \\
& & & 1 & 1 & 1 & 1
\end{tabular} & $\begin{array}{l:l}\vdots & \vdots\end{array}$ & 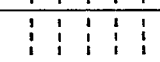 & $180:$ & 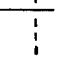 \\
\hline $\cos \theta \cos$ & $\mathrm{z}$ & $\cos \cos c \cos \theta$ & $\Leftrightarrow \sim z_{1}$ & 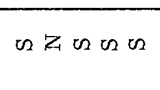 & 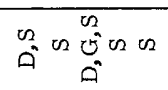 & $\infty$ \\
\hline $33 \sum_{109}^{3}$ & K & 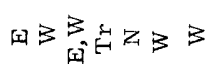 & $3: 3$ & 的石 & : & 问 \\
\hline 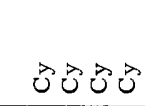 & Z & 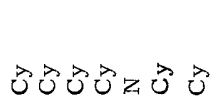 & 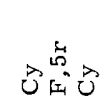 & 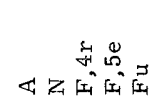 & 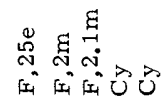 & $\theta$ \\
\hline 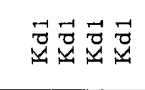 & $\overrightarrow{0}$ & 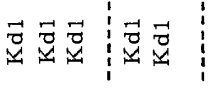 & 式吾苟 & $\mid \begin{array}{ll}\vec{\sigma} \vec{\sigma} \\
\vdots\end{array}$ & 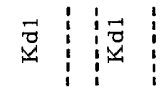 & \\
\hline 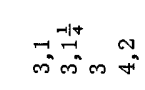 & m & 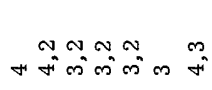 & $\begin{array}{l}-1+\infty \\
\infty \pi\end{array}$ & 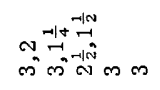 & 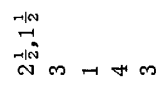 & $\stackrel{\sim}{\infty}$ \\
\hline 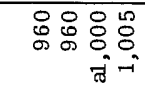 & 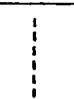 & 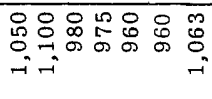 & 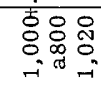 & 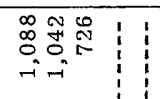 & 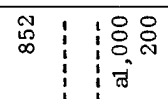 & $\underset{\infty}{\infty}$ \\
\hline 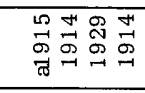 & 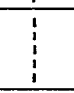 & 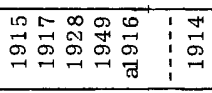 & 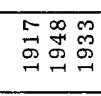 & 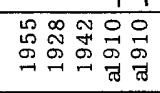 & 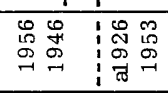 & 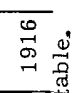 \\
\hline 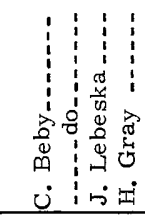 & $\begin{array}{r}\vdots \\
\vdots \\
\vdots \\
\vdots \\
0 \\
0 \\
0 \\
0 \\
0\end{array}$ & 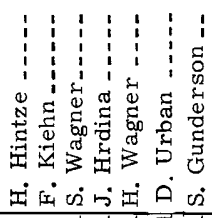 & 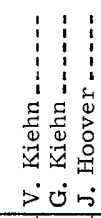 & 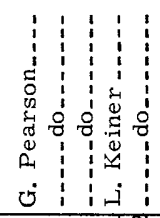 & 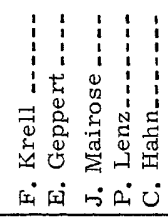 & 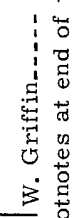 \\
\hline 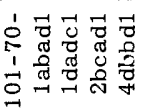 & 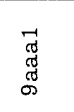 & 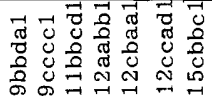 & 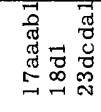 & 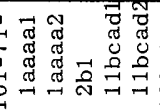 & 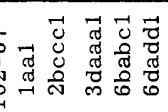 & 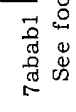 \\
\hline
\end{tabular}




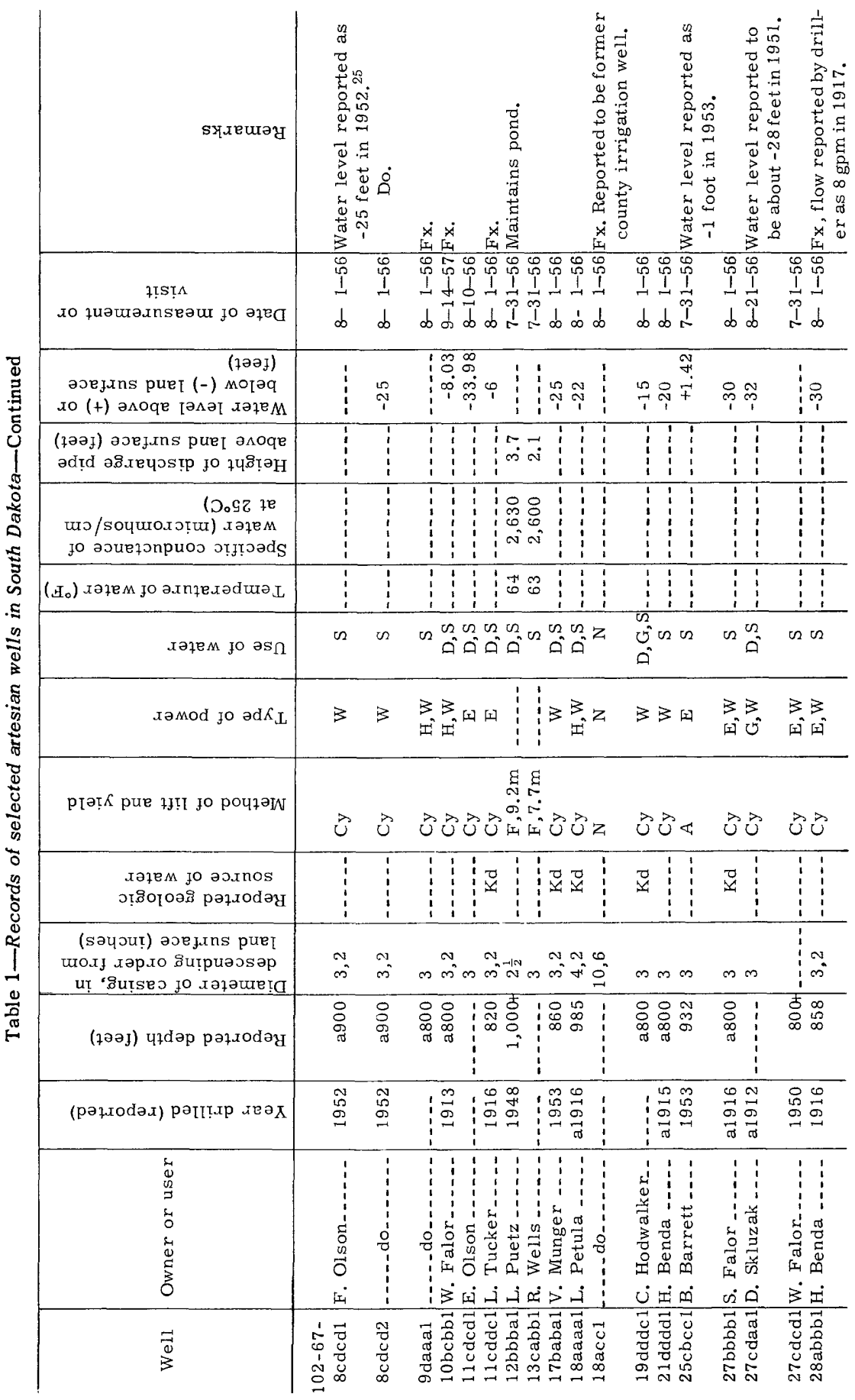


0
0
0
0
0
0
0
0
0
0
0
0
0
है
0
0
0
0

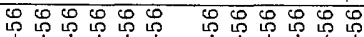

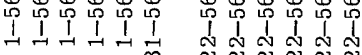

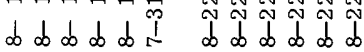
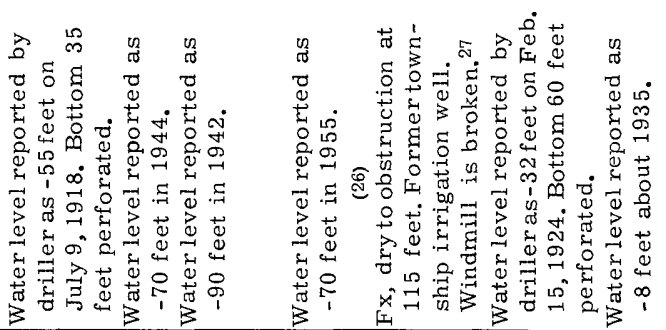

\begin{tabular}{|c|c|c|c|c|c|c|c|c|c|}
\hline 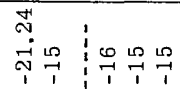 & $\begin{array}{llll} & 1 & & \\
1 & 1 & 8 \\
1 & 1 & 1 & 1\end{array}$ & $\begin{array}{ll}1 & 1 \\
1 & 1 \\
1 & 1 \\
1 & 1\end{array}$ & : & $\begin{array}{l}0 \\
? \\
0 \\
0 \\
1\end{array}$ & 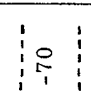 & 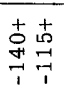 & $\begin{array}{l}1 \\
\vdots \\
\vdots\end{array}$ & $\stackrel{\infty}{1}_{1}^{\infty}$ & i \\
\hline $\begin{array}{llllll}1 & 1 & 1 & 1 & 1 & 1 \\
1 & & 1 & 1 & & 1 \\
1 & 1 & 1 & 1 & 1 & 1 \\
\end{array}$ & $\begin{array}{llll} & 1 & 1 & 1 \\
& 1 & & 1 \\
1 & 1 & 1 & 1 \\
\end{array}$ & & i & 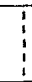 & $\begin{array}{l:c} & 1 \\
1 & 1 \\
1 & 1 \\
1 & 1 \\
\end{array}$ & $\begin{array}{l:}1 \\
\vdots \\
\vdots \\
1 \\
1\end{array}$ & $\begin{array}{c}1 \\
1 \\
1 \\
1\end{array}$ & $\begin{array}{l}1 \\
\vdots \\
1\end{array}$ & $\begin{array}{l}1 \\
1 \\
1 \\
1\end{array}$ \\
\hline $\begin{array}{llllll}1 & 1 & 1 & 1 & 1 & 1 \\
& 1 & 1 & & 1 & 1 \\
1 & 1 & 1 & 1 & 1 & 1 \\
1 & 1 & 1 & & 1 & 1 \\
1 & 1 & 1 & 1 & 1 & 1 \\
\end{array}$ & $\begin{array}{llll} & 1 & 1 & 1 \\
1 & 1 & 1 & 1 \\
1 & 1 & 1 & 1 \\
1 & 1 & 1 & 1\end{array}$ & $\begin{array}{l}1 \\
\vdots \\
1 \\
1\end{array}$ & $\begin{array}{l}1 \\
1 \\
1 \\
1\end{array}$ & $\begin{array}{l}1 \\
\vdots \\
\vdots \\
\vdots\end{array}$ & $\begin{array}{l:c}1 & \vdots \\
1 & 1 \\
1 & 1 \\
1 & 1 \\
1 & 1 \\
1 & 1\end{array}$ & 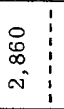 & i & $\begin{array}{l}1 \\
\vdots \\
\vdots \\
i\end{array}$ & $\begin{array}{l}1 \\
\vdots \\
1 \\
1\end{array}$ \\
\hline $\begin{array}{lllllll} & 1 & 1 & 1 & 1 & 1 \\
& 1 & & 1 & & 1 \\
1 & 1 & 1 & 1 & 1 & 1\end{array}$ & $\begin{array}{llll} & 1 & 1 & \vdots \\
1 & 1 & 1 & 1\end{array}$ & $\begin{array}{ll}1 \\
1 \\
1\end{array}$ & $i$ & $\begin{array}{l}i \\
\vdots\end{array}$ & $\begin{array}{lll} & \vdots & 1 \\
1 & 1 & 1\end{array}$ & $\begin{array}{ll}1 \\
\vdots \\
1 & 1\end{array}$ & i & i & 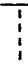 \\
\hline 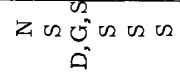 & 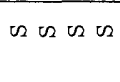 & $a^{2}$ & $\stackrel{0}{a}$ & 乙 & $\pi \sim a^{\Omega}$ & $\sim z$ & $\begin{array}{l}\text { क } \\
5 \\
0\end{array}$ & $a^{2}$ & $U 2$ \\
\hline 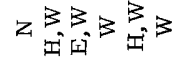 & 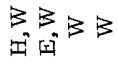 & 3 & 3 & ' & 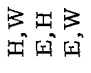 & ${ }_{[x \mid}$ & 3 & 3 & Gi \\
\hline
\end{tabular}

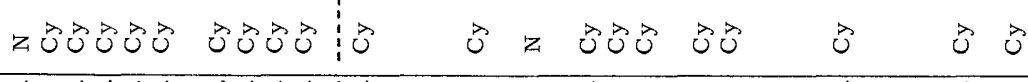

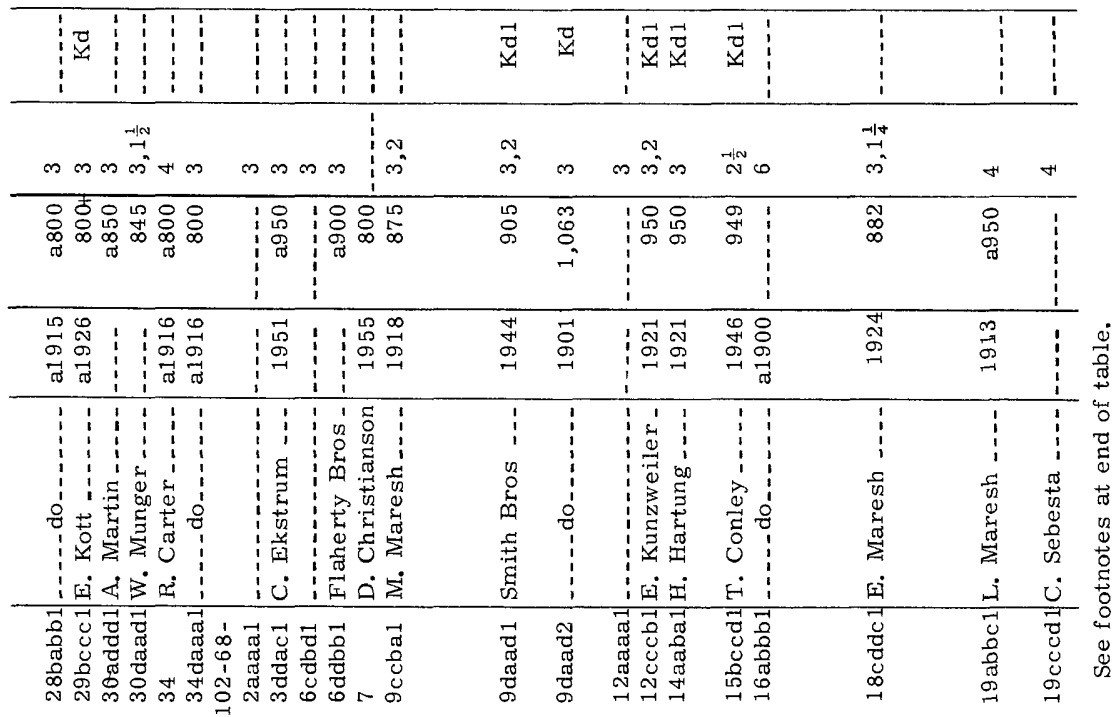




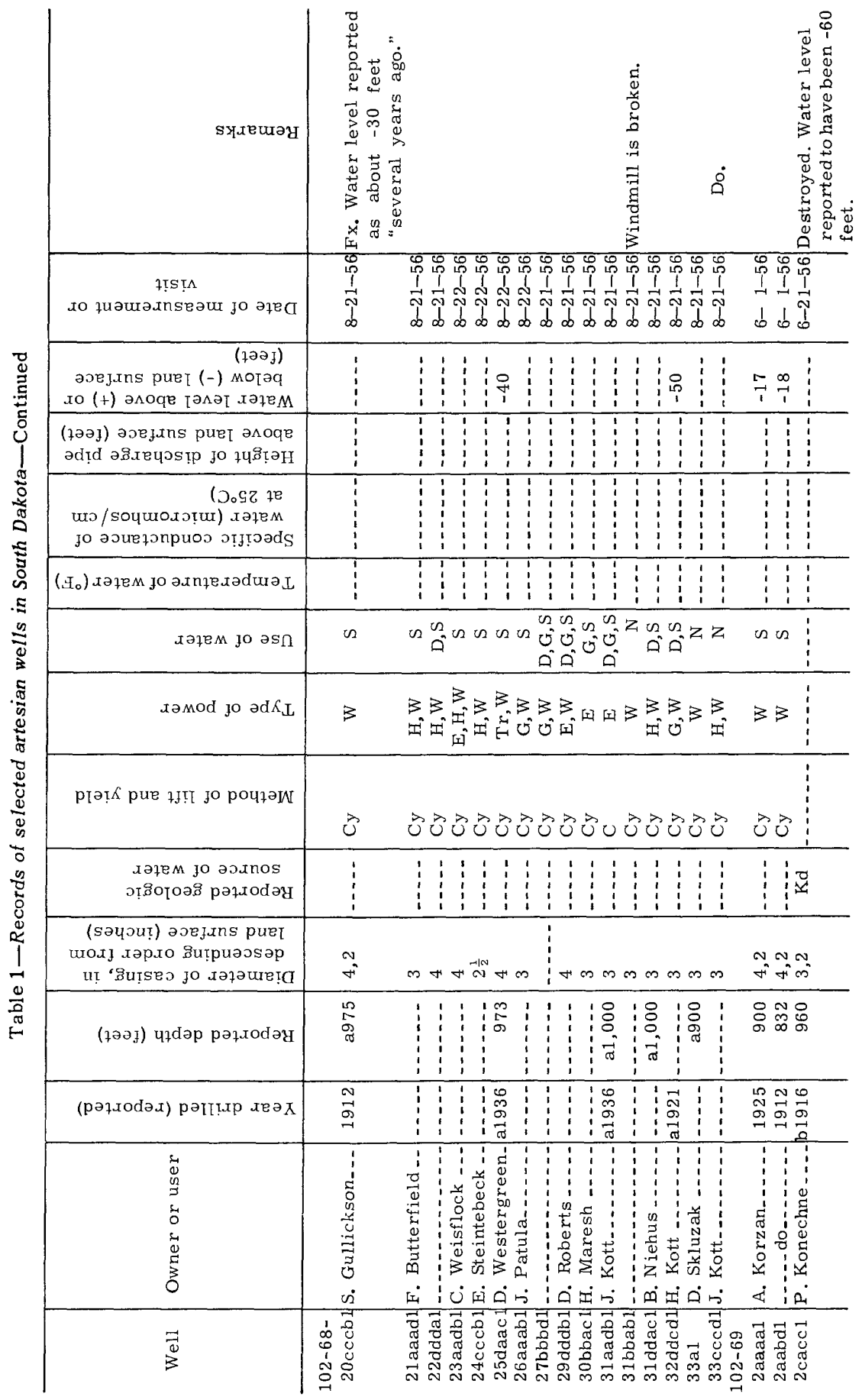




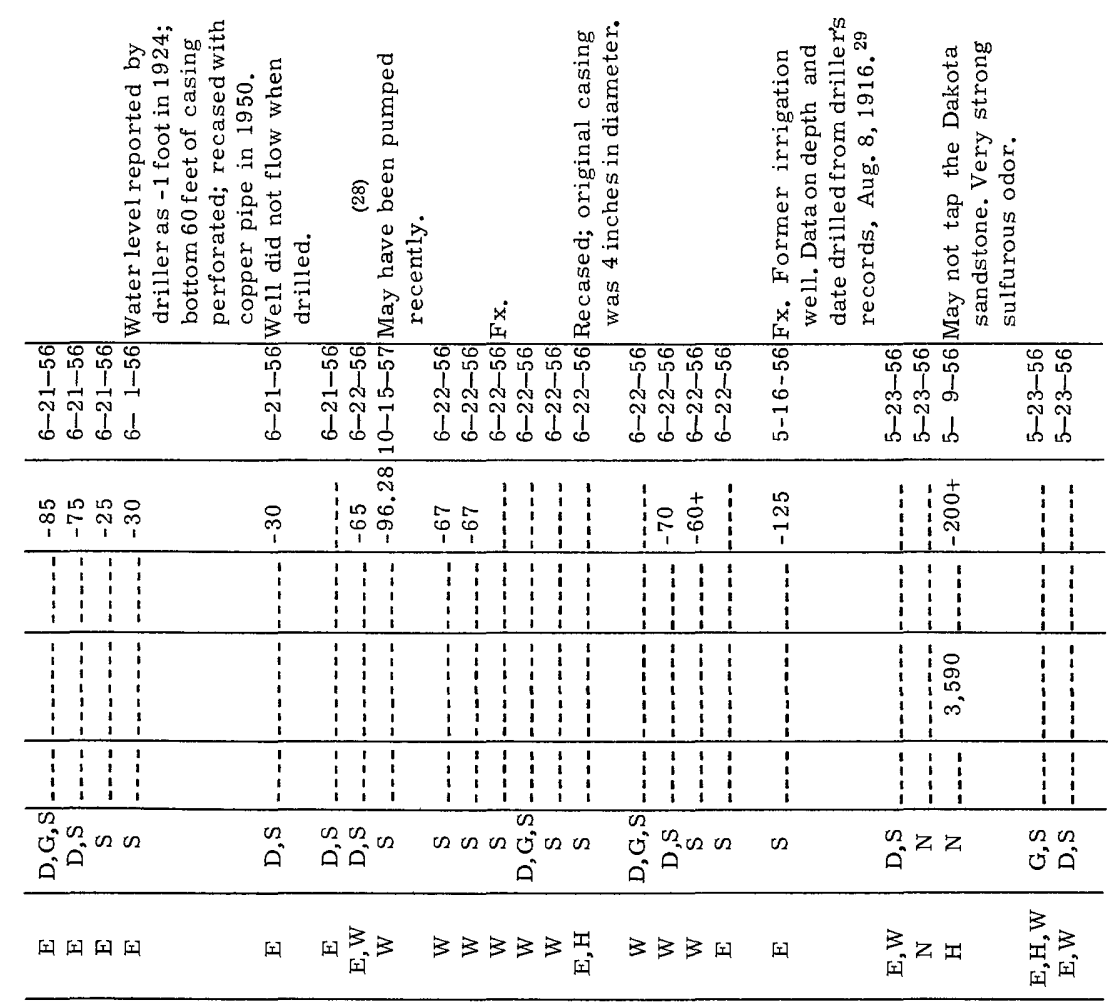

\begin{tabular}{|c|c|c|c|c|c|c|c|}
\hline టेंેे & $\hat{U}$ & $\hat{0} \overrightarrow{0}$ & 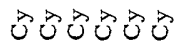 & $\overrightarrow{0} \hat{U} \overrightarrow{0}$ & $r$ & $\hat{U} z \dot{U}$ & $\hat{U}$ \\
\hline $\begin{array}{llll}1 & 1 & 1 & 1 \\
1 & 1 & & 1 \\
1 & 1 & 1 & 1 \\
\end{array}$ & & 层 & 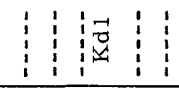 & : & i & 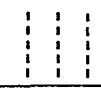 & \\
\hline $\sin \pi{ }^{N}$ & $\stackrel{-14}{\infty}$ & $\stackrel{-1+4}{=}$ & 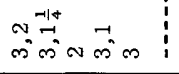 & ๓ ले ले ले & $\infty$ & No & $\pi t$ \\
\hline \begin{tabular}{l:l}
$\infty$ & 0 \\
\hdashline & 0 \\
\hdashline & $\sigma$ \\
\hdashline & 0
\end{tabular} & $\stackrel{H}{\text { N }}$ & 용్ㅀ స్ & 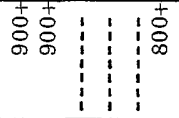 & 离令 & 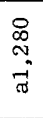 & \begin{tabular}{ll|}
$\infty$ & 0 \\
$\infty$ & 8 \\
$\infty$ & 8
\end{tabular} & $\begin{array}{l}\circ \\
\circ\end{array}$ \\
\hline 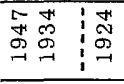 & $\begin{array}{l}\infty \\
\vec{\sigma} \\
\rightarrow\end{array}$ & 아요요 & 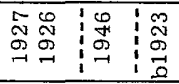 & $\begin{array}{lll}0 & 10 \\
0 & 0 \\
\sigma & 5 \\
-1 & 0 \\
\end{array}$ & $\begin{array}{l}\infty \\
\infty \\
\infty \\
\infty \\
\infty\end{array}$ & 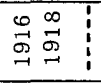 & 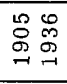 \\
\hline 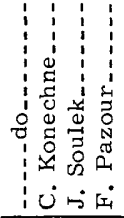 & 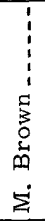 & 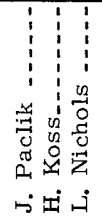 & 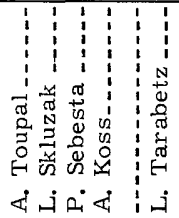 & 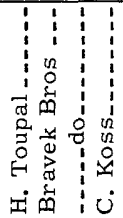 & 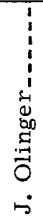 & 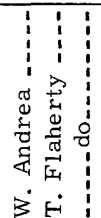 & 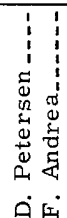 \\
\hline 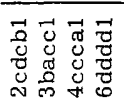 & 要 & 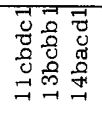 & 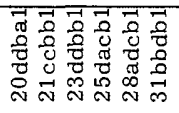 & 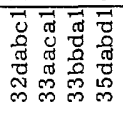 & & 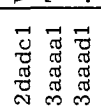 & 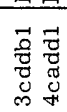 \\
\hline
\end{tabular}




\begin{tabular}{|c|c|c|c|c|}
\hline Sy.jeury & 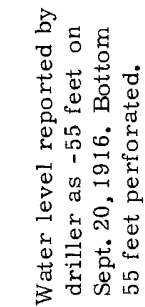 & 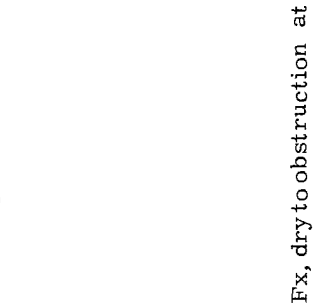 & 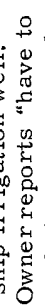 & \\
\hline 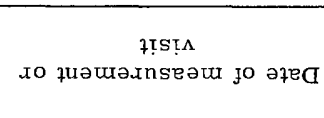 & $\begin{array}{l}0 \\
1 \\
1 \\
0 \\
\text { ஸे } \\
1\end{array}$ & 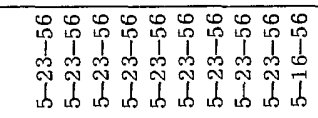 & 感 & 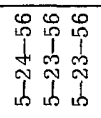 \\
\hline 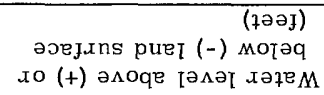 & $\vdots$ & $\mathrm{i} i \mathrm{i} i$ & $\stackrel{8}{i}$ & $\vdots$ \\
\hline 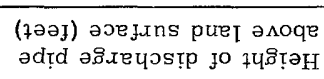 & $\vdots$ & $\begin{array}{lllll}i & 1 & 1 & 1 & 1\end{array}$ & $\vdots$ & \\
\hline 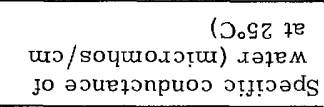 & 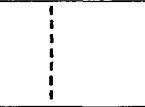 & 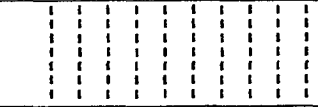 & $\begin{array}{c}1 \\
\vdots \\
\vdots \\
\vdots \\
\vdots \\
\end{array}$ & \\
\hline 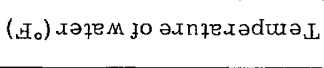 & $\vdots$ & 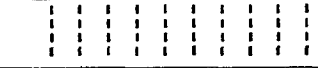 & í & $\begin{array}{lcc}1 & 1 & 1 \\
& 1 & 1 \\
& 1 & 1 \\
1 & 1 & \\
\end{array}$ \\
\hline хәұем јо әs & z & 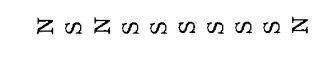 & in & $\operatorname{an} 0^{2}$ in \\
\hline хәмод јо әd $K_{\amalg}$ & 3 & 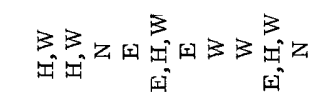 & $\begin{array}{l}3 \\
\text { 仿 }\end{array}$ & 的菃B \\
\hline 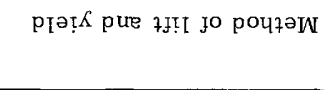 & $\overrightarrow{0}$ & 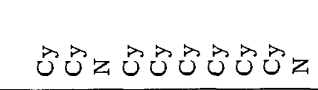 & $\overrightarrow{0}$ & $\overrightarrow{U U}$ \\
\hline 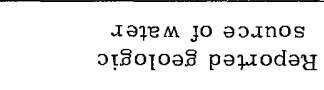 & $\vdots$ & 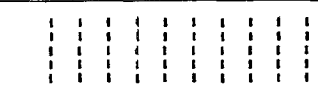 & $\overrightarrow{0}$ & 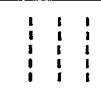 \\
\hline 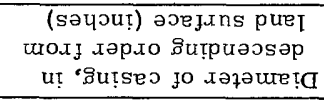 & $\infty$ & HAt & $\pi$ & $\begin{array}{l}H+\infty \\
\because m m\end{array}$ \\
\hline (ұәәј) цұdәр рәұлодәч & 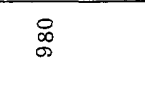 & 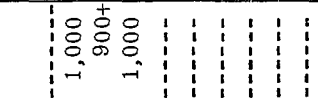 & ஓ & 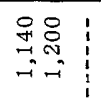 \\
\hline 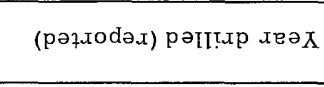 & $\begin{array}{l}0 \\
-1 \\
-1\end{array}$ & 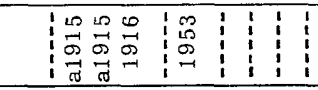 & $\underset{\sim}{\stackrel{\leftrightarrow}{\sim}}$ & 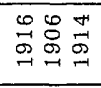 \\
\hline 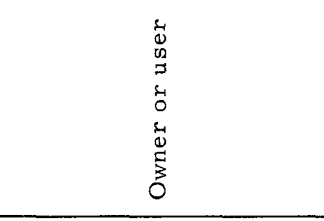 & $\begin{array}{c}1 \\
\vdots \\
1 \\
0 \\
0 \\
0 \\
0 \\
0 \\
0 \\
0 \\
\dot{0} \\
\dot{0}\end{array}$ & 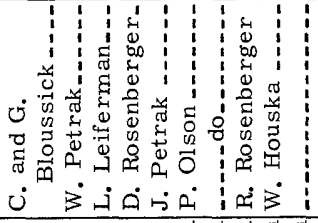 & 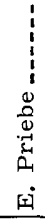 & 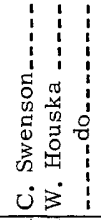 \\
\hline$\sum_{3}^{7}$ & $\begin{array}{l}1 \\
0 \\
0 \\
1 \\
0 \\
0 \\
0 \\
0 \\
0\end{array}$ & 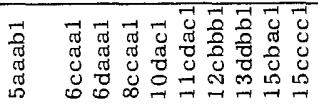 & $\begin{array}{l}\overline{0} \\
0 \\
0 \\
\text { के }\end{array}$ & 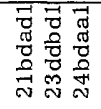 \\
\hline
\end{tabular}




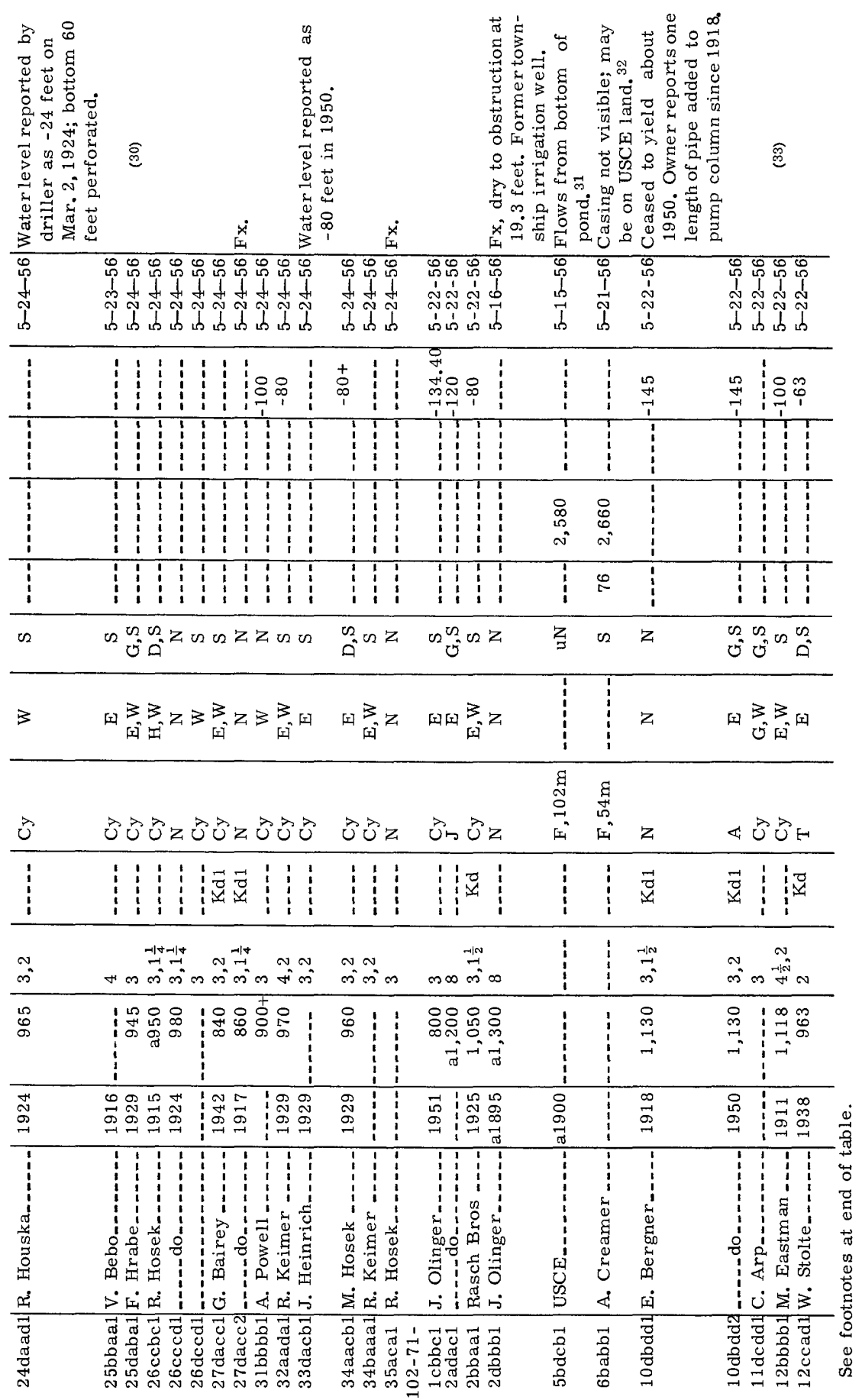




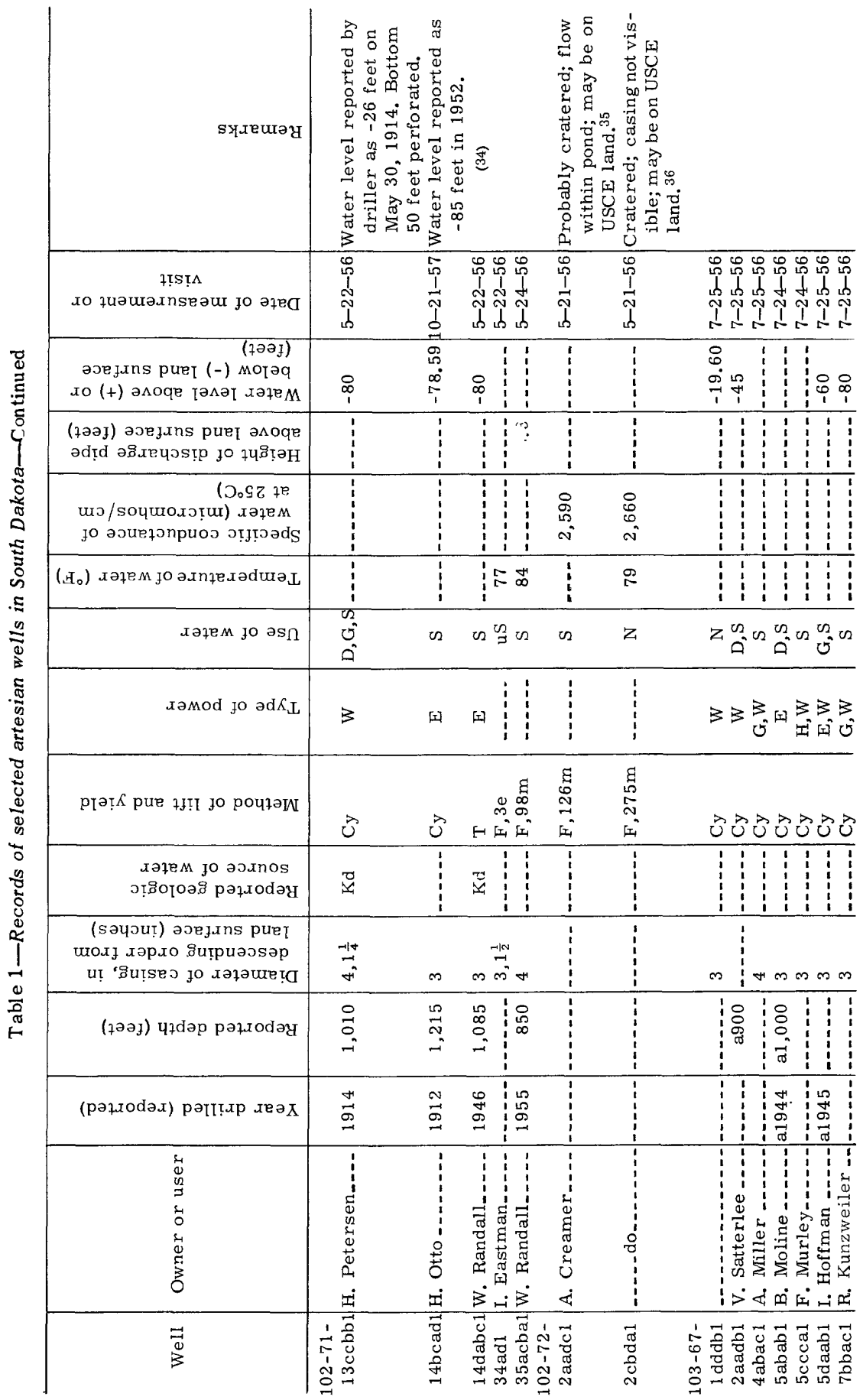




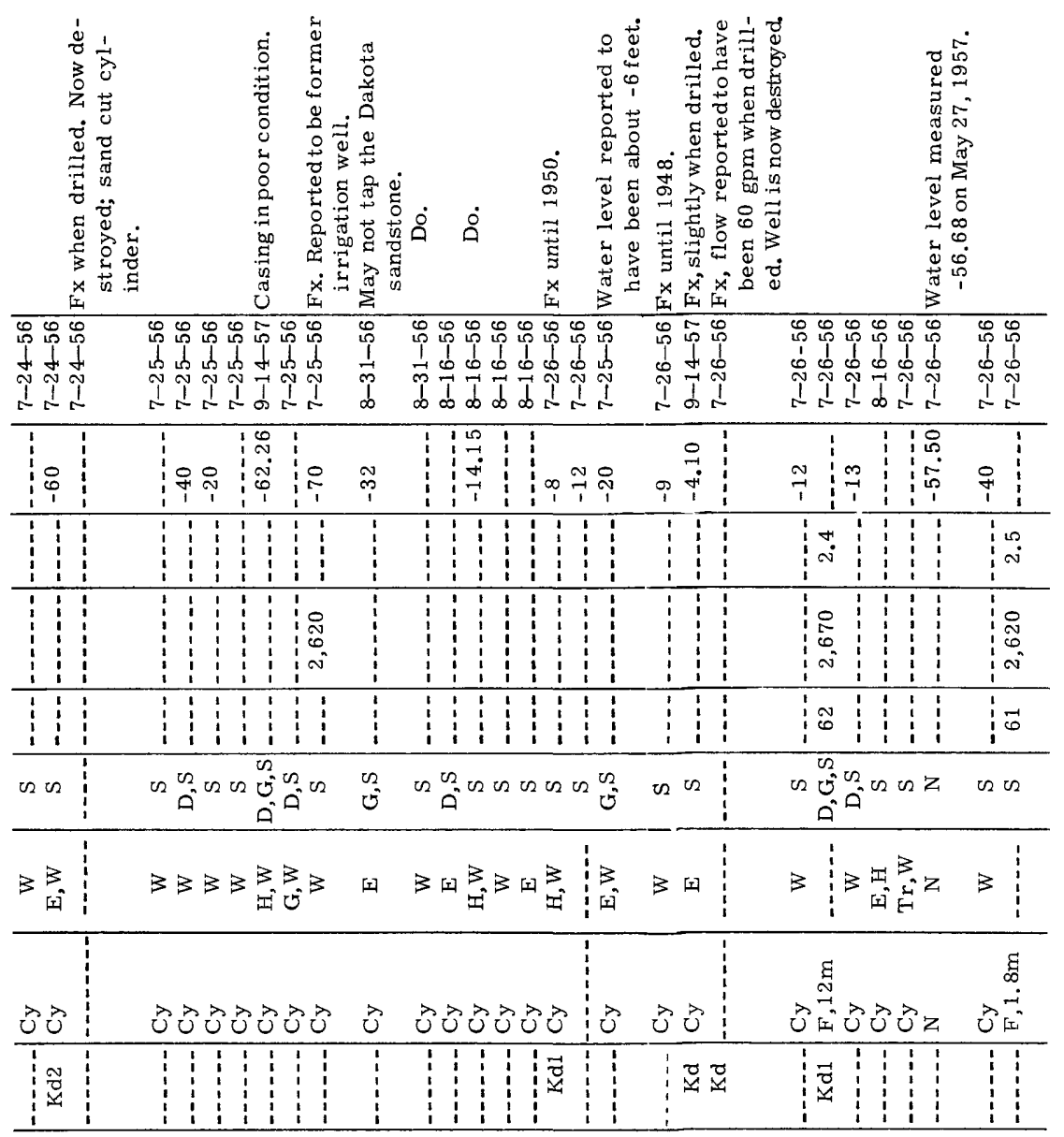

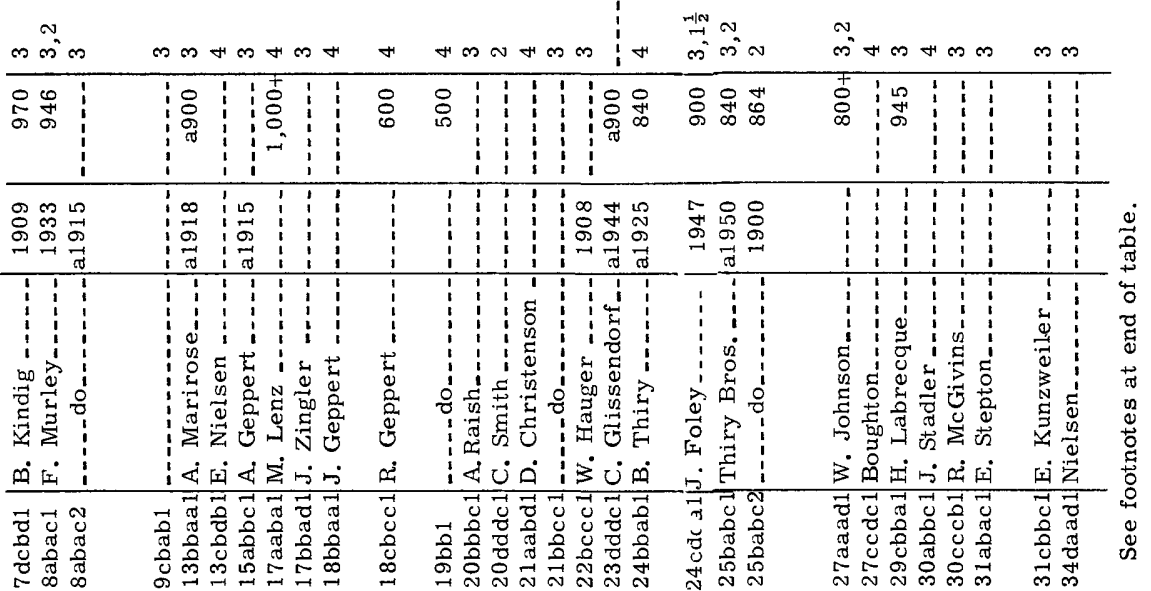




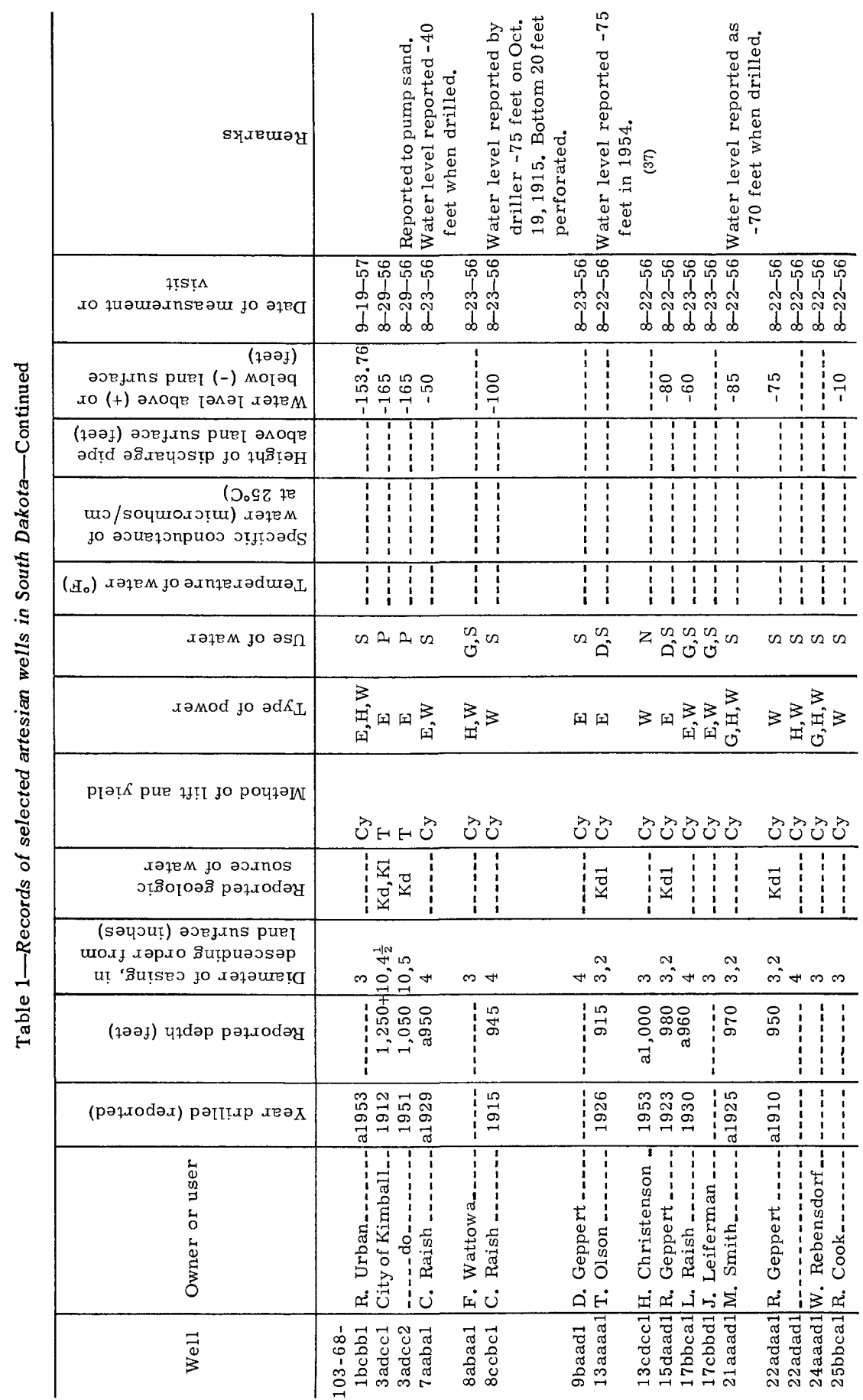




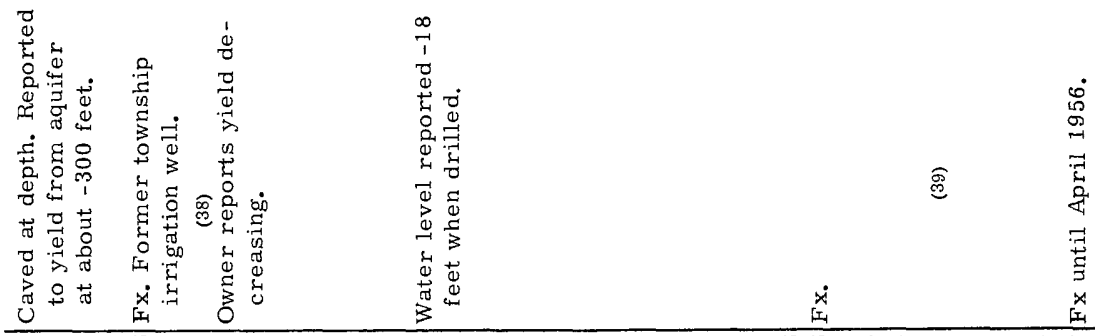

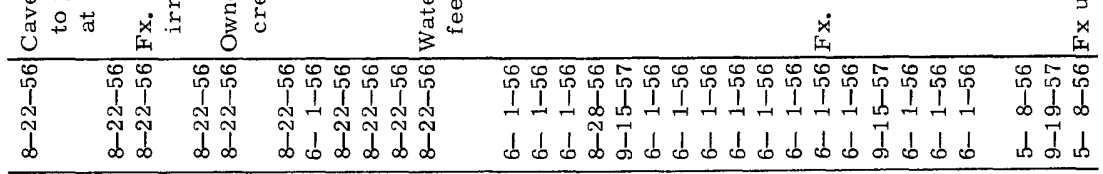

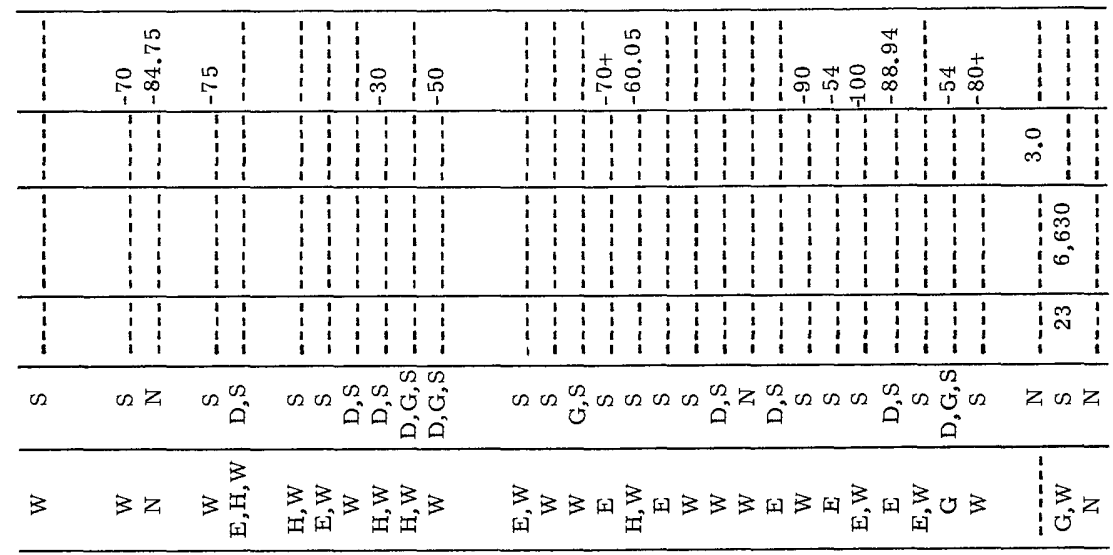

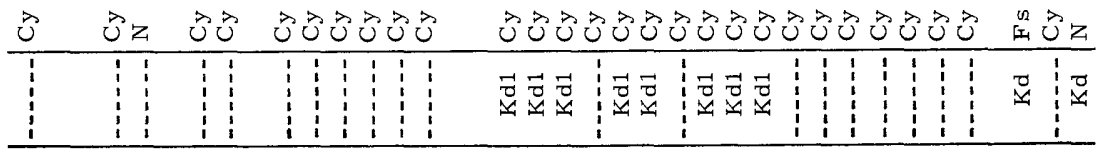

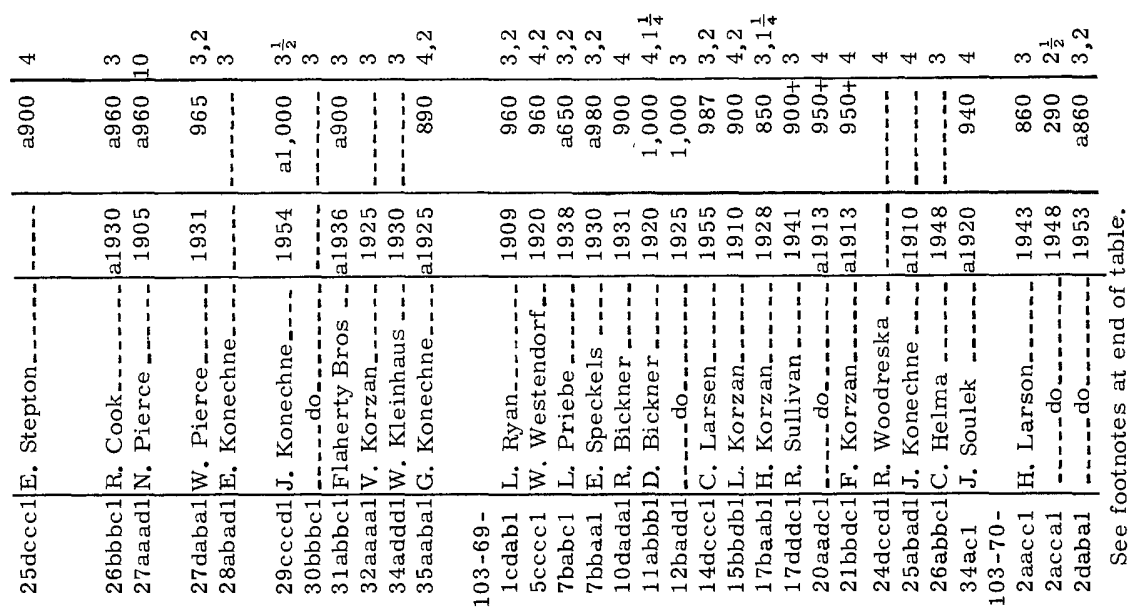




\begin{tabular}{|c|c|c|c|c|c|}
\hline sभлешту & 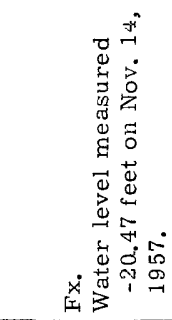 & 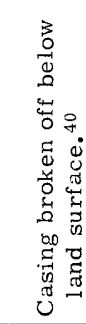 & 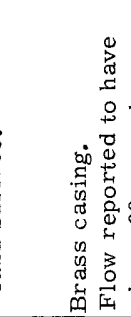 & 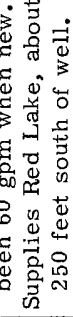 & 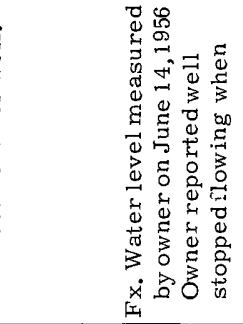 \\
\hline 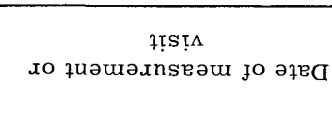 & 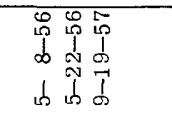 & $\begin{array}{ll}0 & 0 \\
0 & 0 \\
1 & 0 \\
0 & 1 \\
0 & 0 \\
1 & 1 \\
0 & 1\end{array}$ & 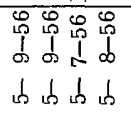 & $\begin{array}{c}\stackrel{0}{0} \\
\stackrel{1}{2} \\
1 \\
10\end{array}$ & $\begin{array}{lllll}0 & 0 & 0 & 0 & 0 \\
0 & 0 & 0 & 0 & 0 \\
0 & 1 & 1 & 1 & 1 \\
0 & 1 & 0 & 0 & 0 \\
1 & 1 & 1 & 1 & 1 \\
10 & 1 & 1 & 1\end{array}$ \\
\hline 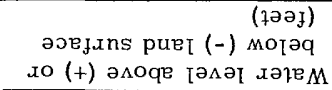 & 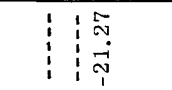 & $\begin{array}{l}i \\
\vdots \\
i \\
i\end{array}$ & 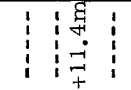 & $\vdots$ & 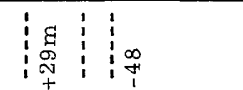 \\
\hline 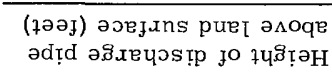 & $\begin{array}{c:c:}\infty & \vdots \\
-i & :\end{array}$ & $\begin{array}{l:l} & \cdots \\
\cdots & \vdots\end{array}$ & 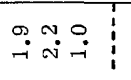 & 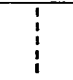 & 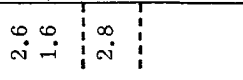 \\
\hline 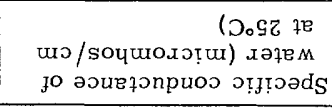 & 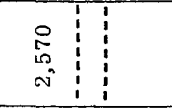 & 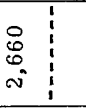 & 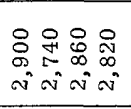 & 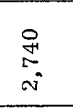 & 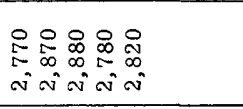 \\
\hline 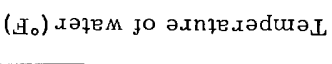 & $\begin{array}{l:l}\sim & 1 \\
\omega & 1\end{array}$ & $\therefore$ & 둗ㅇㅇㅛ & $\stackrel{p}{\sim}$ & 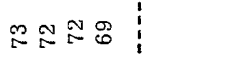 \\
\hline xәұем јо әs $\Omega$ & en & 的 & 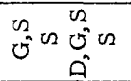 & -1 & 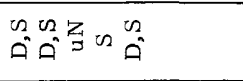 \\
\hline ләмOd fo $ә \mathrm{~d} \kappa_{\mathrm{L}}$ & $\begin{array}{l:l}1 & 0 \\
1 & 1\end{array}$ & 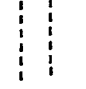 & $\begin{array}{llll} & 1 & 1 & 1 \\
1 & & 1 & 1 \\
1 & 1 & 1 & 1\end{array}$ & 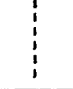 & 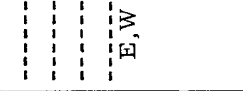 \\
\hline 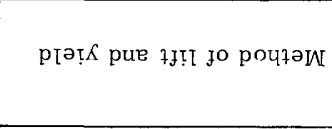 & $\begin{array}{l:l} & \\
G & \\
O & \\
N & \\
I_{1}^{n} & 4\end{array}$ & 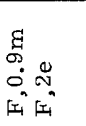 & 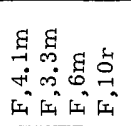 & द्व & 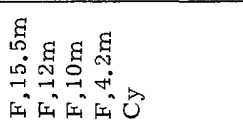 \\
\hline 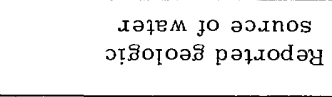 & 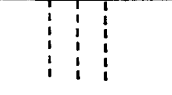 & $\begin{array}{ll}i \\
i & 1 \\
1 & 1 \\
1 & 1\end{array}$ & 可吾 & ขै & 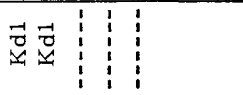 \\
\hline 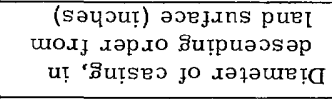 & N & $\rightarrow \cong$ & $N \stackrel{N}{N}$ ल & $\stackrel{\infty}{\infty}$ & $\infty \stackrel{\pi}{\rightarrow+n}$ \\
\hline (ұәәر) чұdәр рәңлоdәч & 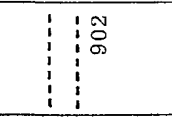 & $\begin{array}{l}i \\
i \\
i \\
i \\
i \\
i \\
i\end{array}$ & $\begin{array}{l}0 \\
\text { 요용 } \\
\infty \\
1\end{array}$ & $\begin{array}{l}0 \\
\stackrel{0}{0} \\
-i\end{array}$ & 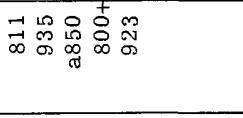 \\
\hline (рәұлоdәл) рәтІтр גеә $\Lambda$ & 粱 & i & 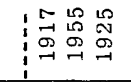 & \begin{tabular}{l}
$\infty$ \\
\multirow{H}{*}{} \\
$\frac{d}{\pi}$
\end{tabular} & 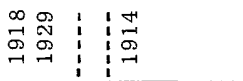 \\
\hline 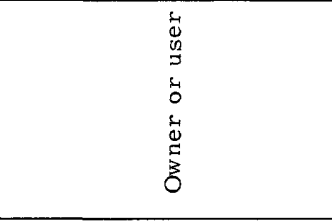 & 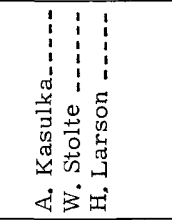 & 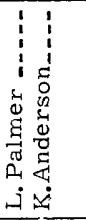 & 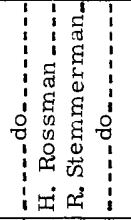 & 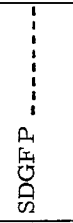 & 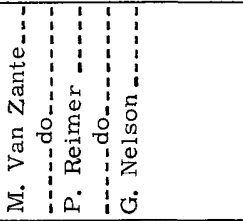 \\
\hline $\overrightarrow{\bar{\omega}}$ & 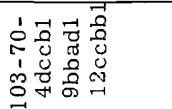 & 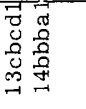 & 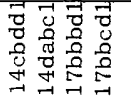 & $\begin{array}{l}\overrightarrow{0} \\
0 \\
0 \\
0 \\
\end{array}$ & 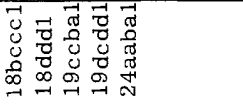 \\
\hline
\end{tabular}




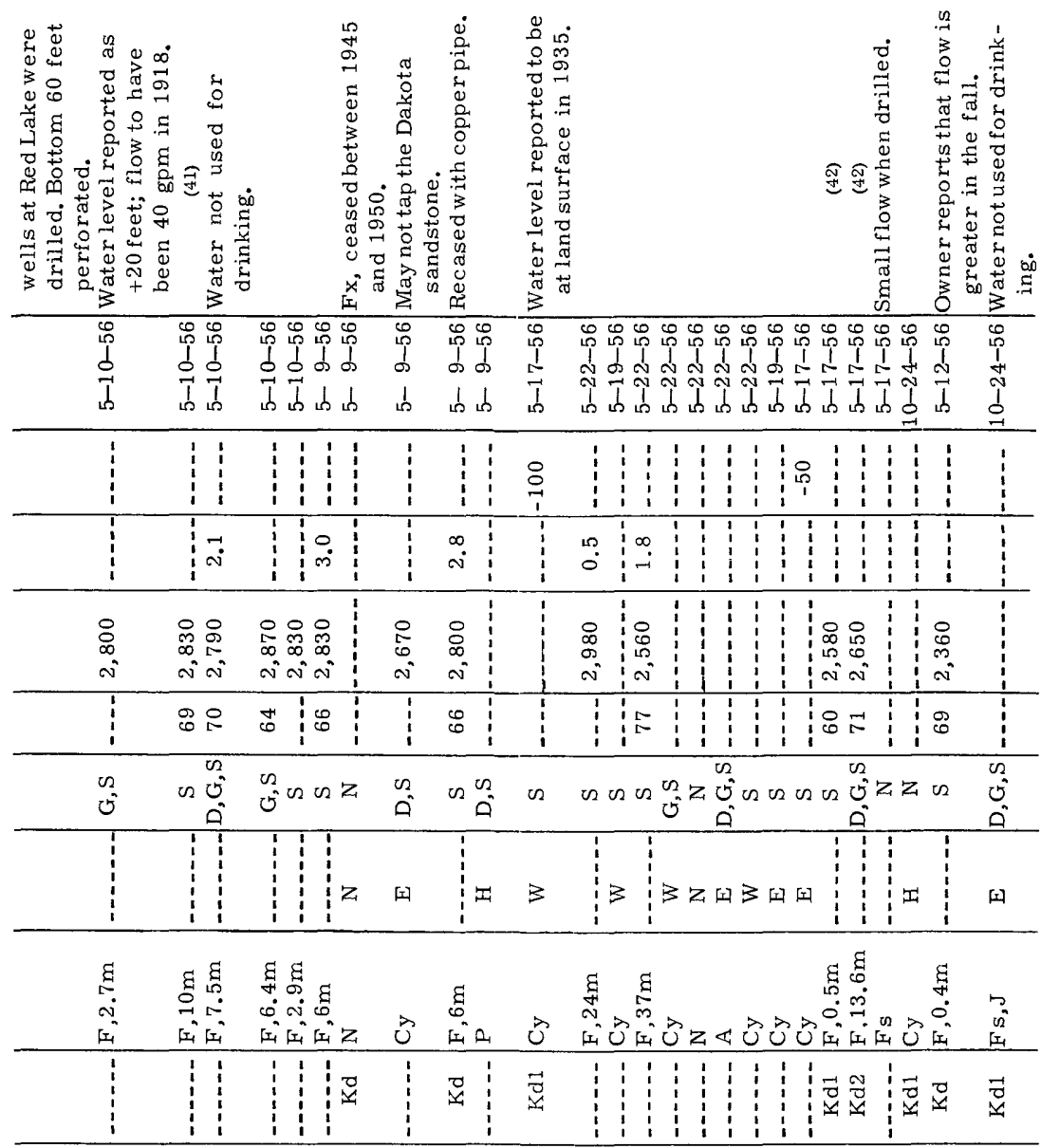

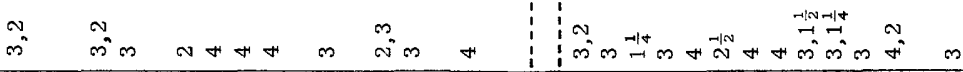

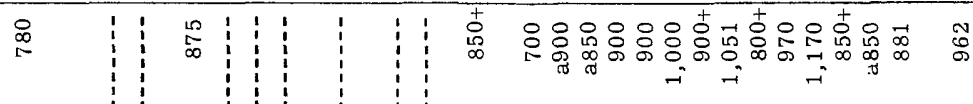

舟

ब

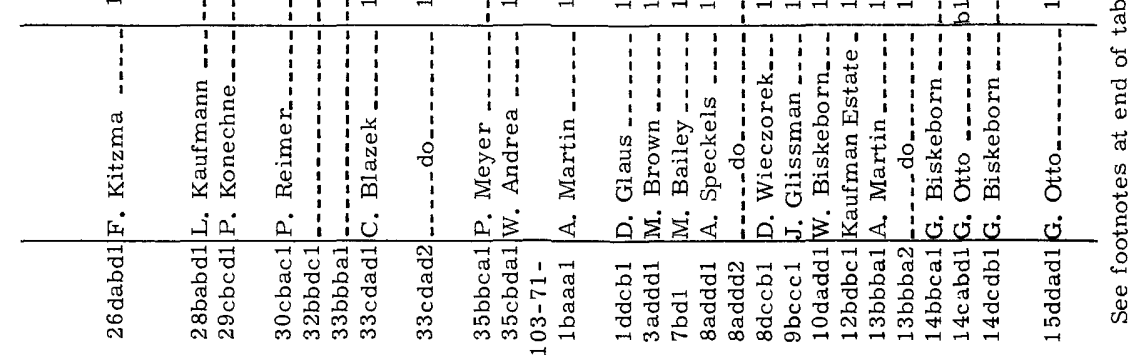




\begin{tabular}{|c|c|c|c|}
\hline syxeüy & 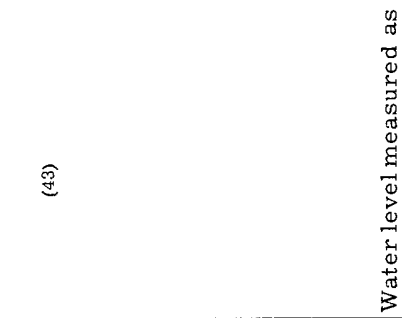 & us & 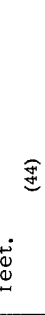 \\
\hline 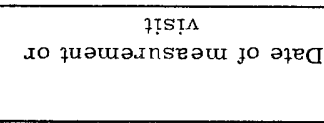 & 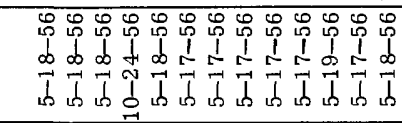 & 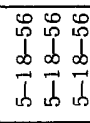 & $\begin{array}{l}0 \\
0 \\
0 \\
\vec{b}\end{array}$ \\
\hline 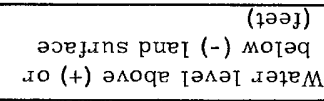 & 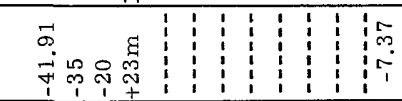 & & 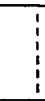 \\
\hline 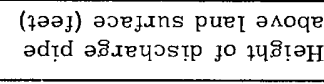 & 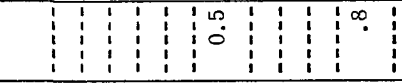 & & 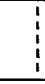 \\
\hline 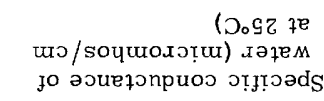 & 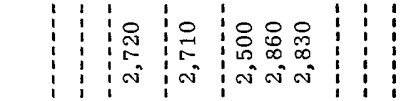 & & \\
\hline$\left(\right.$ ( $\left._{0}\right)$ ) хәңем јо әл пұеләduә & 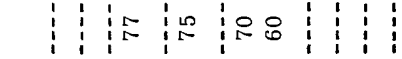 & & 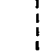 \\
\hline . & 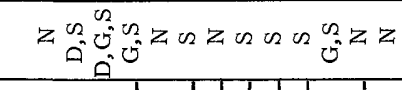 & $\begin{array}{l}\pi^{2} \\
0^{2} z \\
0^{n}\end{array}$ & $z$ \\
\hline ләMOd Jo әd $\kappa_{L}$ & 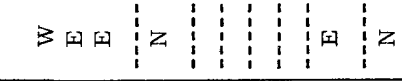 & $33 z$ & 3 \\
\hline 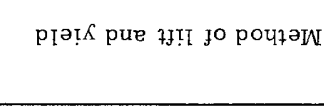 & 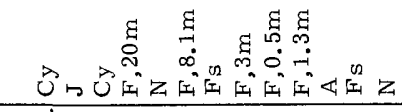 & ठें & Uे \\
\hline 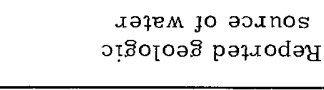 & 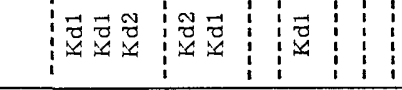 & 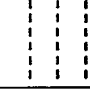 & $\vec{z}$ \\
\hline 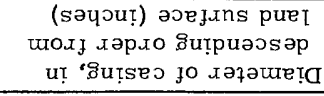 & 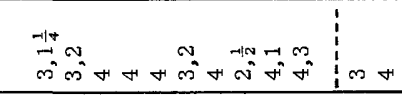 & $\infty \mathrm{m}^{-1} \mathrm{~m}$ & $\vec{m}$ \\
\hline (ұәәј) чұdәр рәұлоdәч & 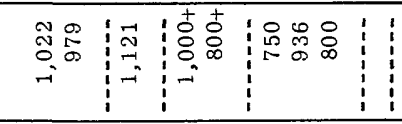 & $\mid \begin{array}{l}0 \\
\vdots \\
0 \\
0\end{array}$ & + \\
\hline 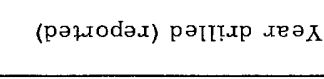 & 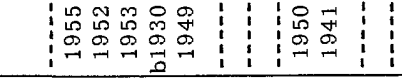 & 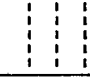 & 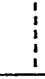 \\
\hline 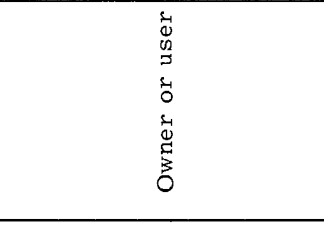 & 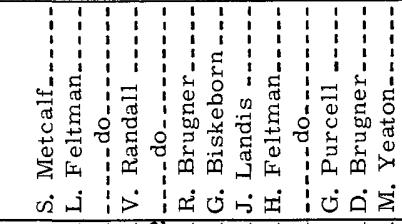 & 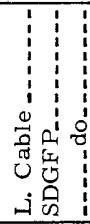 & 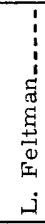 \\
\hline$\overline{0}$ & 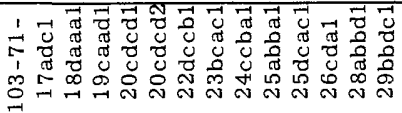 & 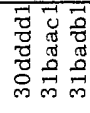 & 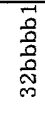 \\
\hline
\end{tabular}




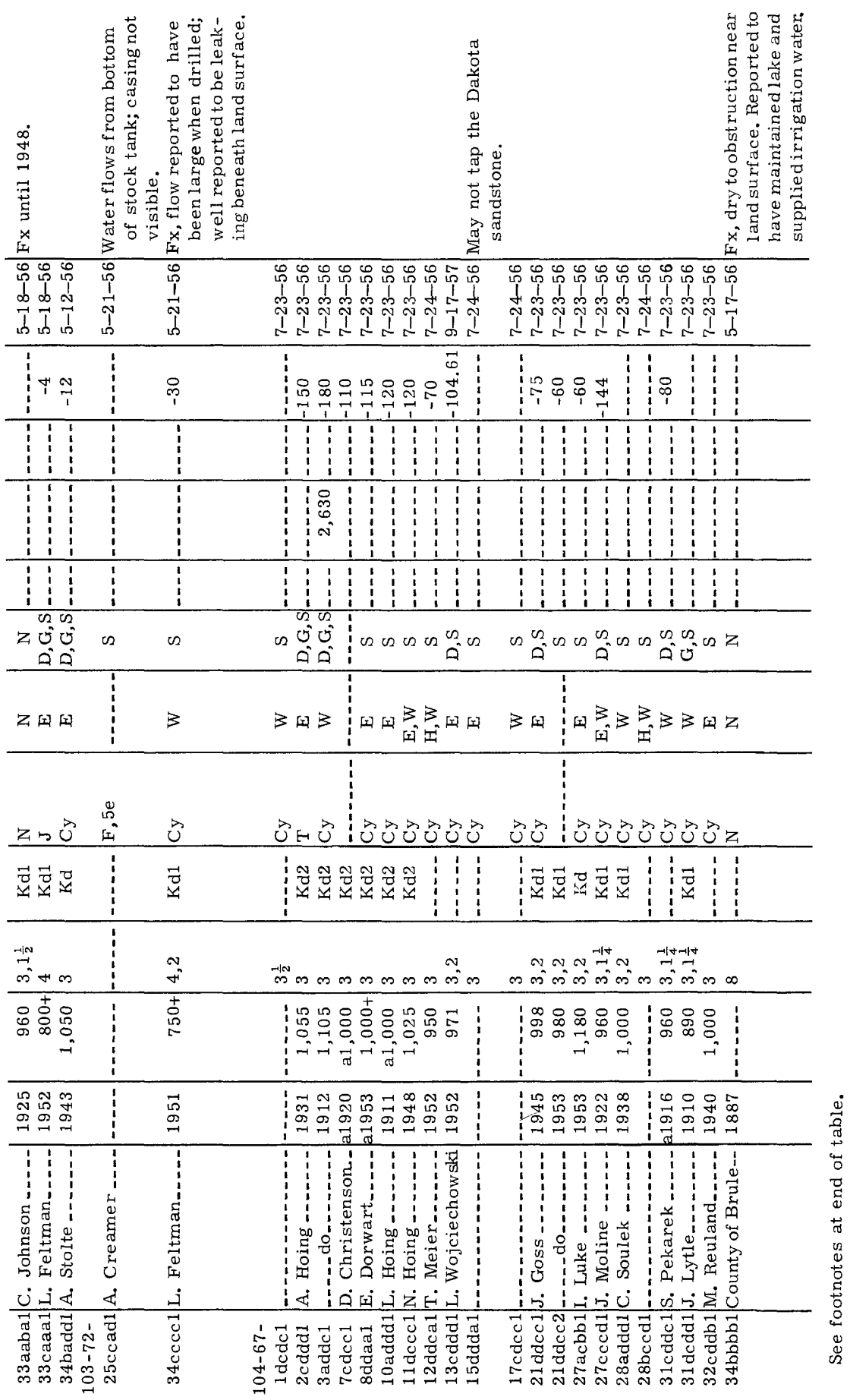




\begin{tabular}{|c|c|c|c|c|}
\hline sңлеuтә & 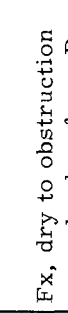 & & 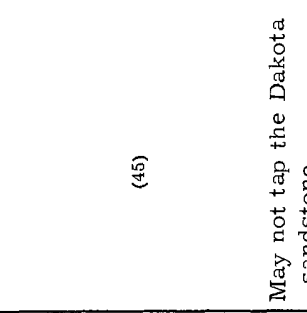 & \\
\hline 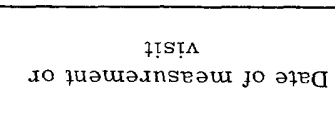 & $\begin{array}{l}0 \\
\stackrel{0}{1} \\
\stackrel{1}{5} \\
\overrightarrow{1} \\
6\end{array}$ & $\begin{array}{l}0 \\
1 \\
\infty \\
i \\
i\end{array}$ & 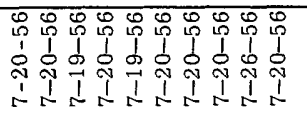 & $\begin{array}{llll}0 & 0 & 0 & 0 \\
0 & 0 & 0 & 0 \\
1 & 1 & 1 & 1 \\
0 & 0 & 0 & 0 \\
\pi & 0 & \pi & 0 \\
1 & 1 & 1 & 1 \\
1 & 1 & 1\end{array}$ \\
\hline 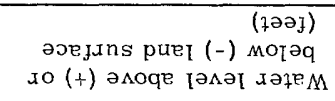 & & 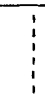 & 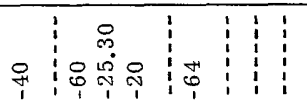 & : \\
\hline 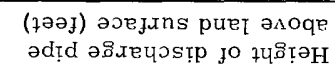 & & 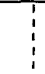 & & \\
\hline 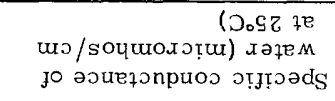 & $\vdots$ & $\vdots$ & & \\
\hline (ㅂ. & $\vdots$ & 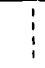 & $\begin{array}{llllllllll}1 & 1 & 1 & 1 & 1 & 1 & 1 & 1 & 1 & 1 \\
1 & 1 & 1 & 1 & 1 & 1 & 1 & & 1 & 1\end{array}$ & 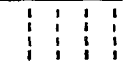 \\
\hline ләMod јо әsก & 乙 & $n$ & 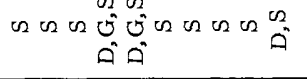 & $\cos 2 \sin ^{2}$ \\
\hline xәMOd јо $ә \mathrm{~d} \kappa_{\mathrm{L}}$ & 乙 & 代 & 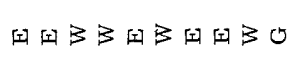 & $\sum_{B=1} B 33$ \\
\hline 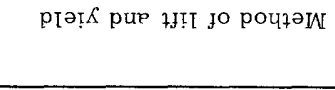 & 乙 & $\vec{v}$ & 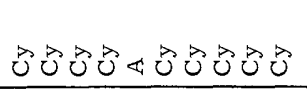 & $\vec{U} \vec{U} \vec{U}$ \\
\hline 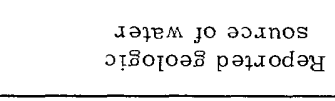 & 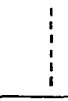 & 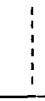 & 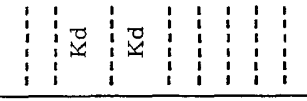 & $\begin{array}{lll:l} & 1 & 1 & \vdots \\
& & & 1 \\
& & & 1 \\
& & 1 & 1 \\
\end{array}$ \\
\hline 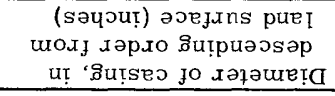 & $\underbrace{-1 / N}_{0}$ & m & Нங & \\
\hline (ұәәј) чұdәр рәұхобәч & 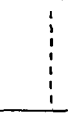 & & 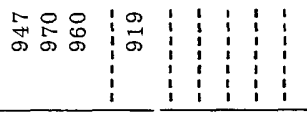 & 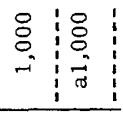 \\
\hline (рәұлодәл) рәाттр леә $\Lambda$ & $\begin{array}{c}\vdots \\
\vdots \\
\vdots \\
\end{array}$ & $\begin{array}{c}\infty \\
\infty \\
\infty \\
-1 \\
-1\end{array}$ & 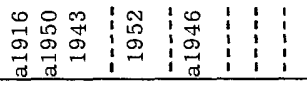 & 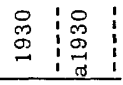 \\
\hline 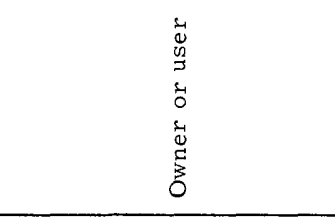 & 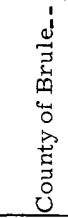 & $\begin{array}{r}\vdots \\
\vdots \\
\vdots \\
0 \\
0 \\
0 \\
0 \\
0 \\
0 \\
\end{array}$ & 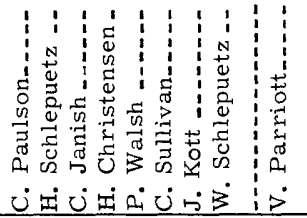 & 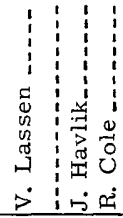 \\
\hline$\frac{\pi}{3}$ & 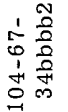 & & 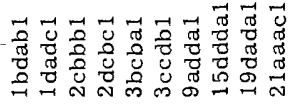 & 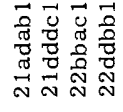 \\
\hline
\end{tabular}




ब. E

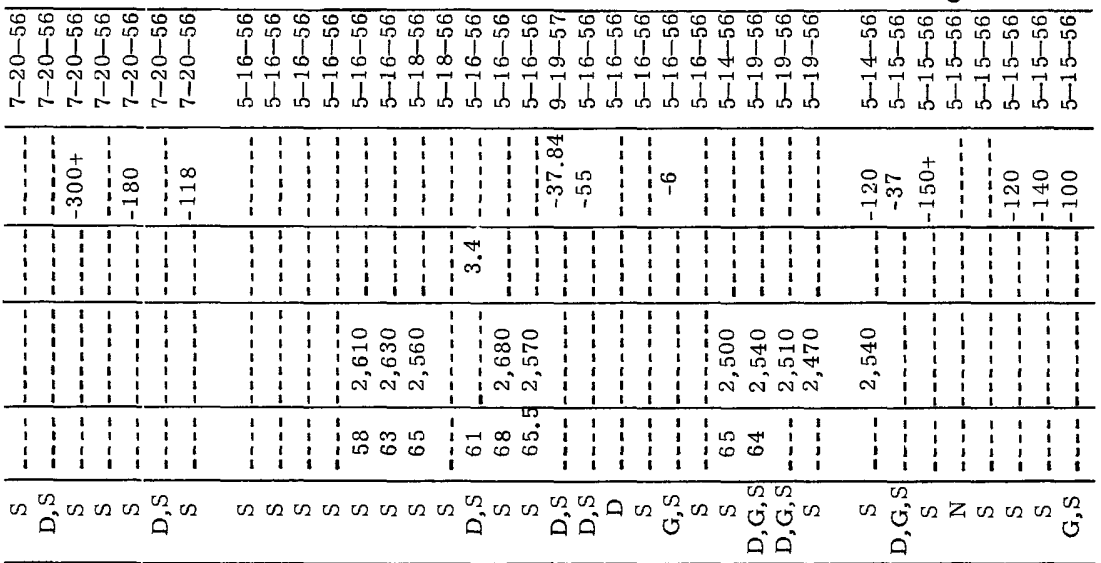

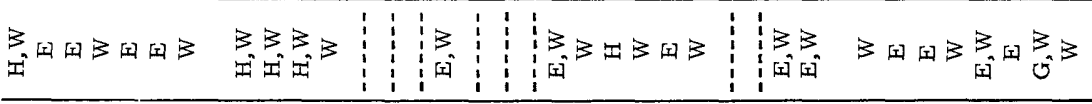

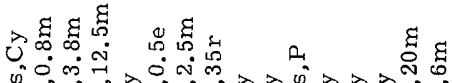

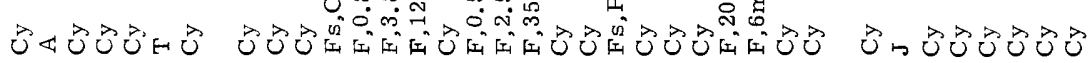

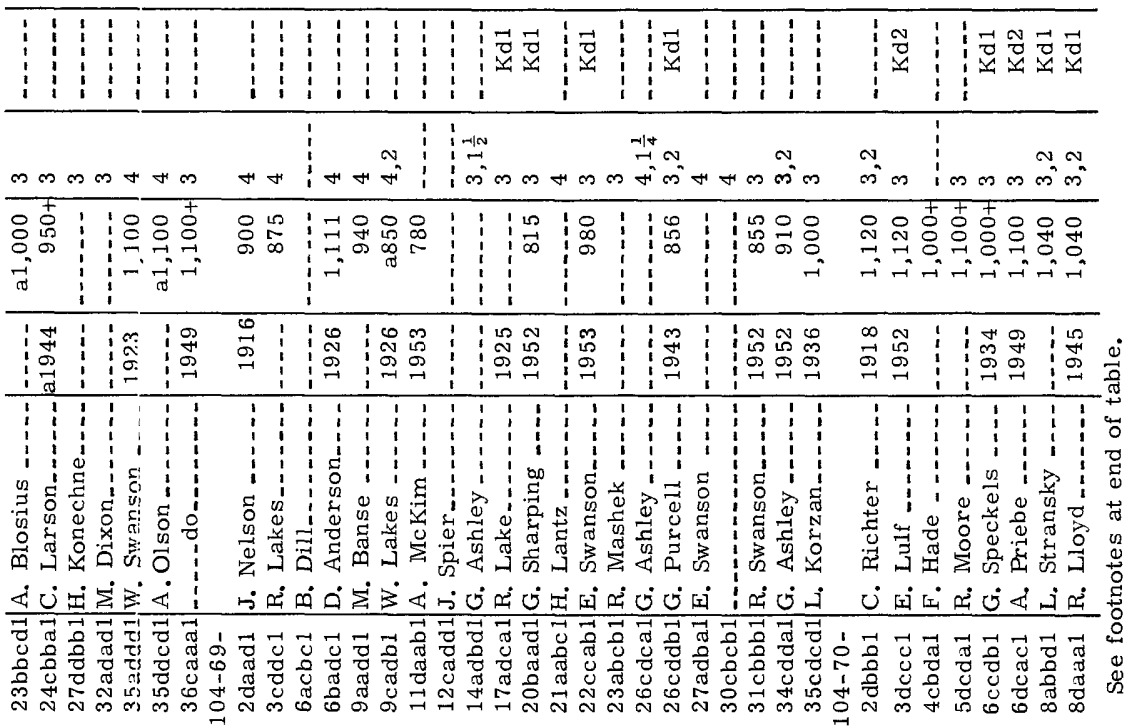




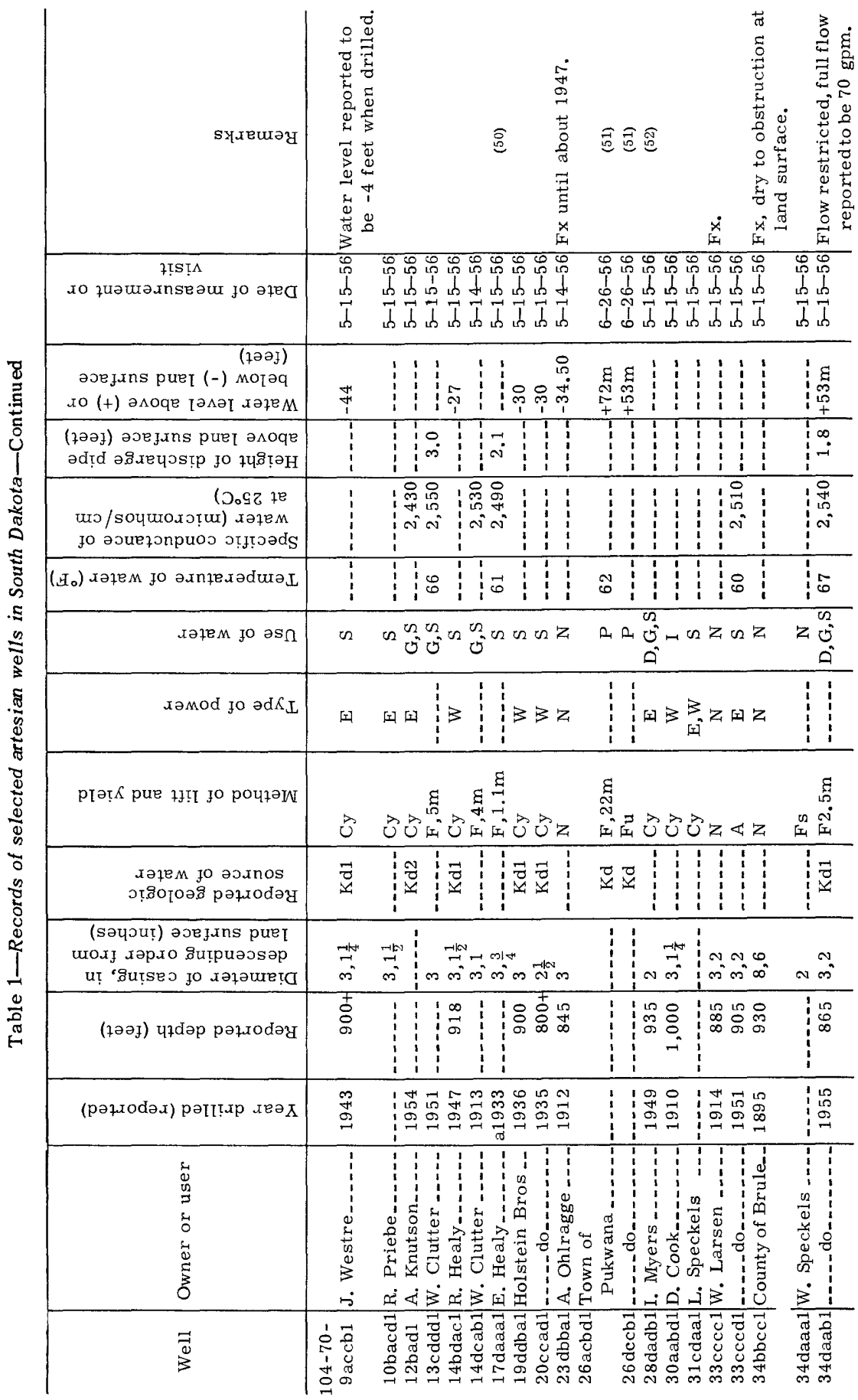




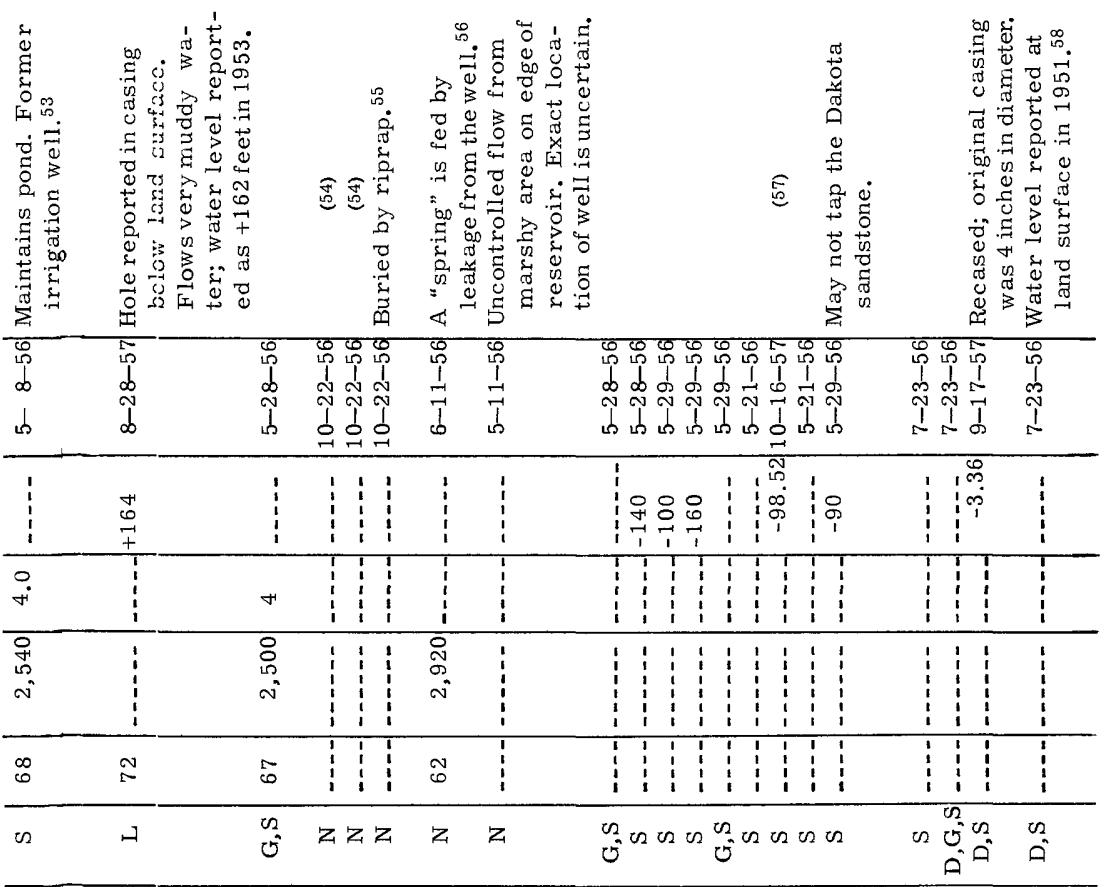

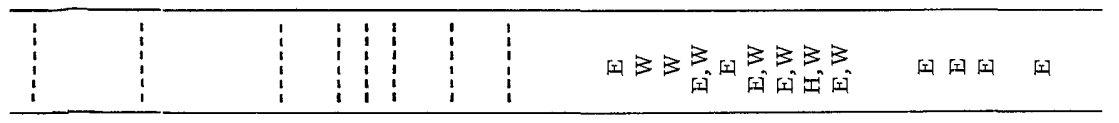

\begin{tabular}{|c|c|c|c|c|c|c|c|c|}
\hline 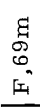 & 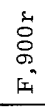 & 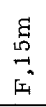 & 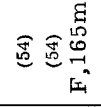 & $\begin{array}{l}\ddot{0} \\
\ddot{0} \\
\dot{0} \\
\dot{1}\end{array}$ & $\begin{array}{l}0 \\
\infty \\
\Sigma^{n}\end{array}$ & 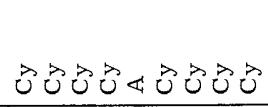 & $\dot{U} 00$ & 4 \\
\hline 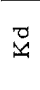 & $\vec{x}$ & ì & \begin{tabular}{ll}
0 \\
\hdashline
\end{tabular} & 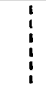 & $\vdots$ & 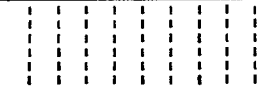 & \begin{tabular}{l|l}
7 & $\vdots$ \\
& $\vdots$
\end{tabular} & 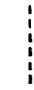 \\
\hline
\end{tabular}

\begin{tabular}{|c|c|c|c|c|c|c|}
\hline 오 & $0^{\infty}$ & + & $\infty \infty$ & $\infty m \infty \in$ & $m \infty N$ & $\stackrel{N}{*}$ \\
\hline 今 & 앙 & $\stackrel{\substack{\infty \\
\infty}}{\stackrel{\infty}{2}}$ & $\begin{array}{l}n \\
\text { ț } \\
0\end{array}$ & 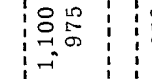 & $\begin{array}{ll}\circ & 1 \\
\Rightarrow & 1\end{array}$ & $\begin{array}{l}0 \\
\infty \\
\infty\end{array}$ \\
\hline
\end{tabular}

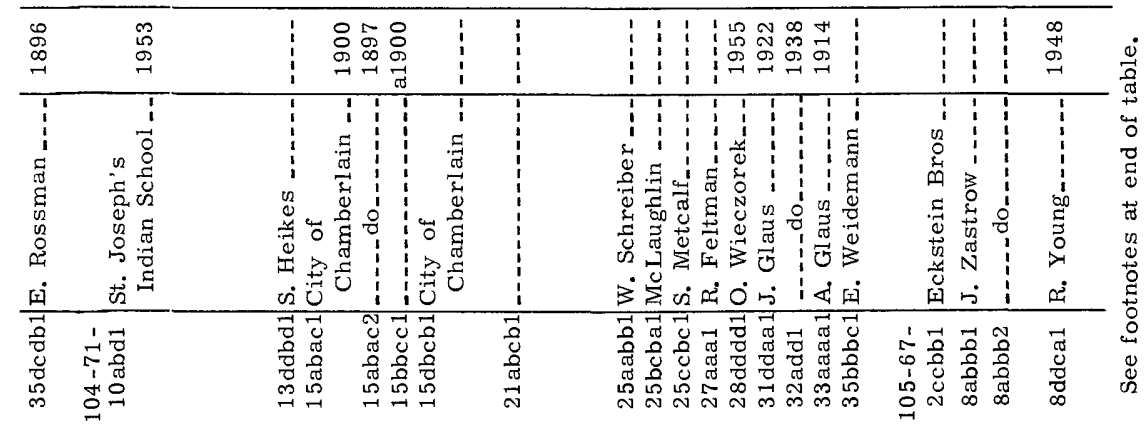




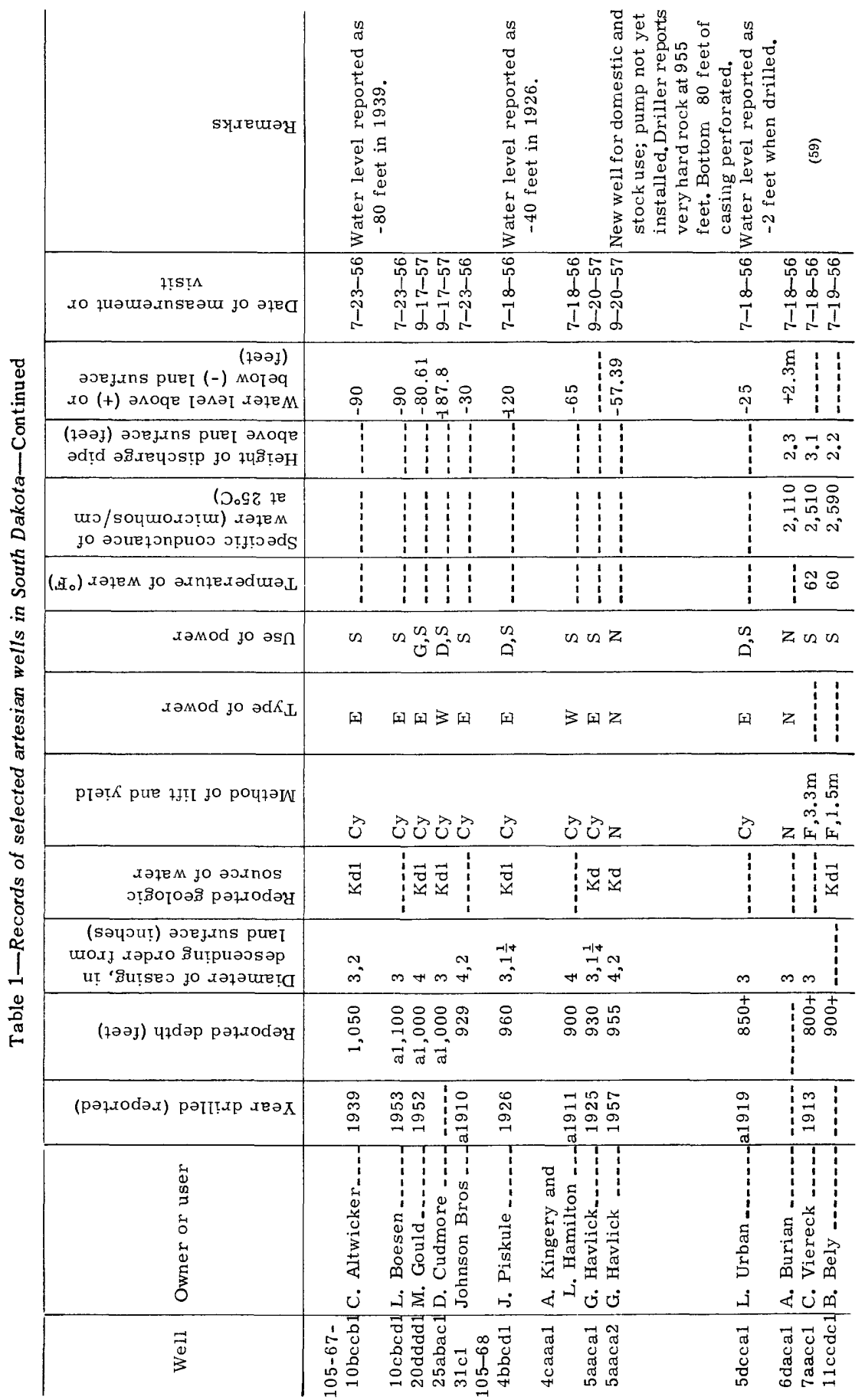




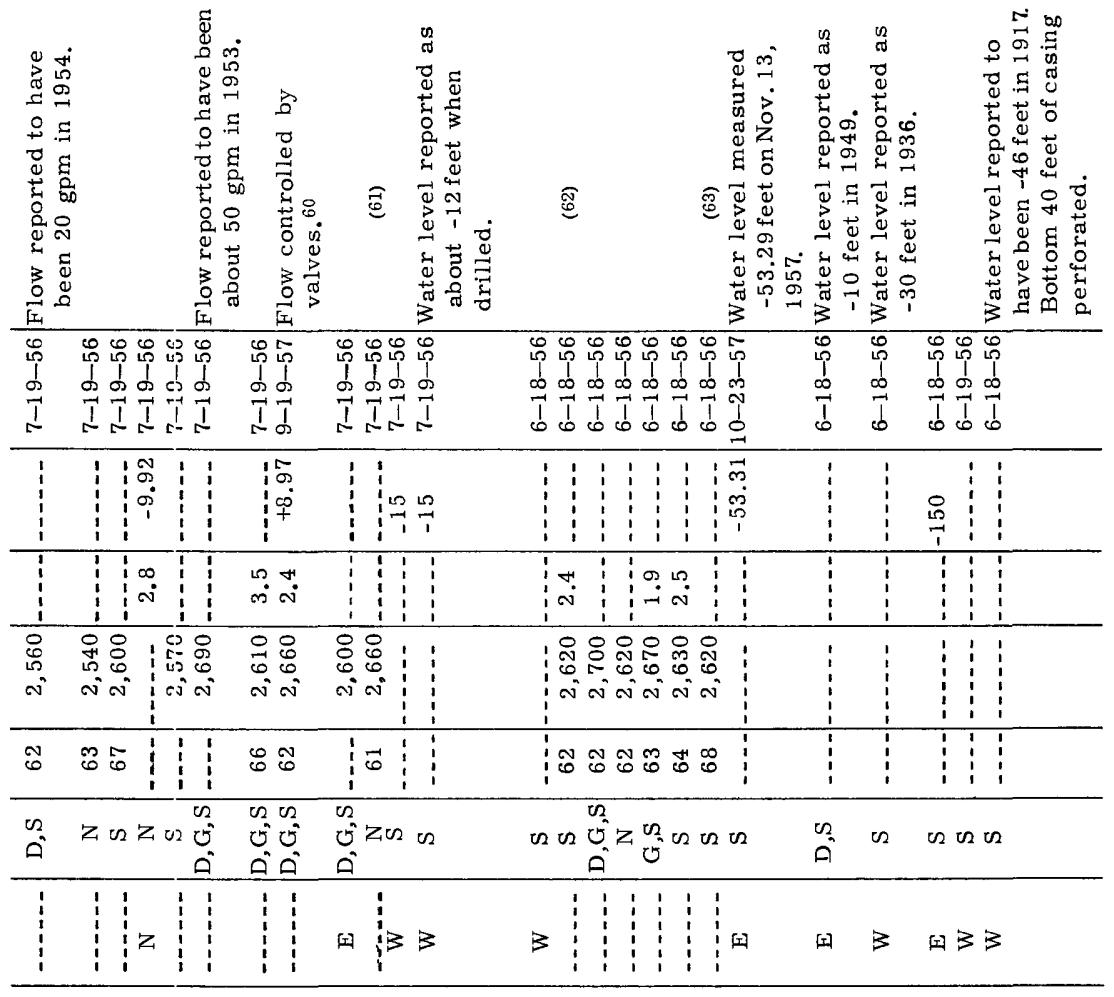

\begin{tabular}{|c|c|c|c|c|c|c|c|}
\hline 茎 & 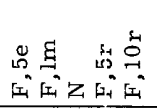 & 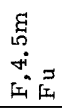 & 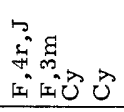 & 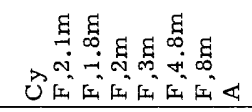 & $\vec{U}$ & $\vec{U}$ & $\varangle \vec{U}$ \\
\hline 8 & & $\vec{z}$ & $\vec{z} \vec{z}$ & 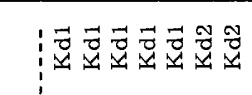 & & ! & \\
\hline$\tilde{n}$ & 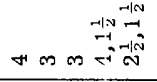 & $+m$ & 蛋的 ñ & 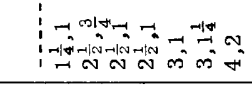 & N & $\stackrel{n}{n}$ & $\tilde{m}^{N} m \tilde{n}^{N}$ \\
\hline $\begin{array}{l}\text { शे } \\
\text { के } \\
\text { ते }\end{array}$ & 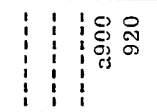 & 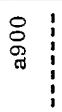 & 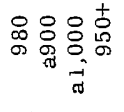 & 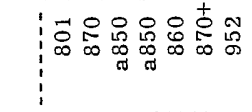 & 号 & $\stackrel{0}{0}$ & 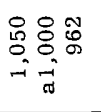 \\
\hline 点 & 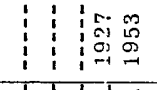 & $\begin{array}{l:}0 \\
\text { D } \\
\text { के } \\
\text { के } \\
\end{array}$ & 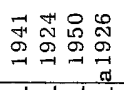 & 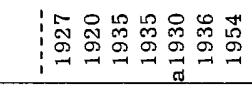 & 罗 & $\stackrel{\sigma}{\sigma}$ & 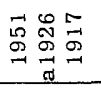 \\
\hline : & 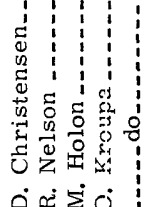 & 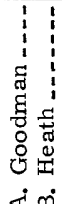 & 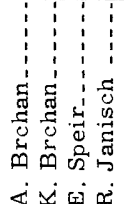 & 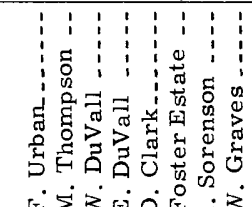 & : & $\begin{array}{c}\vdots \\
\vdots \\
\vdots \\
\vdots \\
\vdots \\
0 \\
0-1 \\
0.1\end{array}$ & 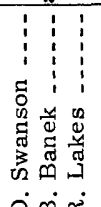 \\
\hline$\dot{\theta}$ & $\dot{A} \dot{\Sigma} 0^{\circ}$ & $\varangle \dot{\varphi}$ & 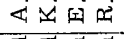 & 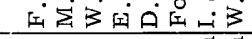 & 4 & ji & $\dot{0} \dot{\varphi x}$ \\
\hline 趂 & 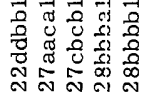 & 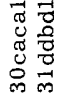 & 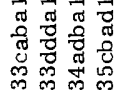 & 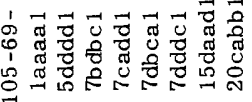 & $\begin{array}{l}\text { है } \\
\text { के } \\
\text { ते }\end{array}$ & 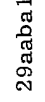 & 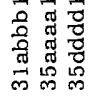 \\
\hline
\end{tabular}




\begin{tabular}{|c|c|c|c|c|c|c|c|c|c|}
\hline sя.дешәч & 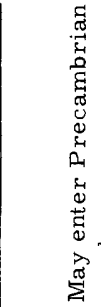 & 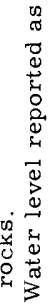 & 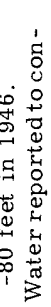 & 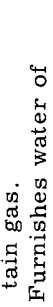 & 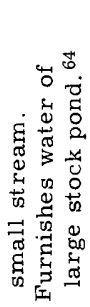 & 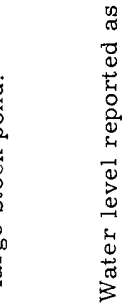 & 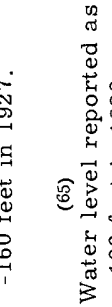 & & \\
\hline 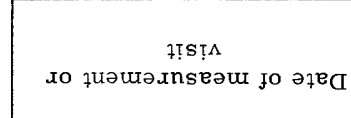 & $\begin{array}{ll}0 & 0 \\
0 & 0 \\
1 & 1 \\
\sim & \stackrel{1}{1} \\
1 & 1 \\
0 & 0\end{array}$ & $\begin{array}{l}0 \\
0 \\
0 \\
0 \\
1 \\
0\end{array}$ & 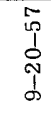 & 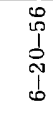 & $\begin{array}{l}0 \\
p \\
1 \\
0 \\
1 \\
0\end{array}$ & 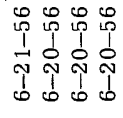 & $\begin{array}{lll}0 & 0 & 0 \\
0 & 0 & 0 \\
1 & 0 & 1 \\
& 0 & 1 \\
1 & 1 & 1 \\
0 & 1 & 0\end{array}$ & $\begin{array}{l}\tilde{0} \\
\text { ஸे } \\
\text { dे }\end{array}$ & 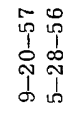 \\
\hline 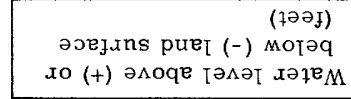 & ల) & $\begin{array}{c}1 \\
\vdots \\
\vdots \\
\vdots\end{array}$ & 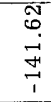 & 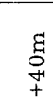 & 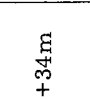 & \begin{tabular}{l:l}
1 \\
\hdashline \\
\hdashline
\end{tabular} & 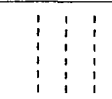 & \begin{tabular}{l}
$\stackrel{8}{N}$ \\
\multirow{5}{0}{} \\
1 \\
1
\end{tabular} & 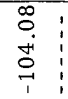 \\
\hline 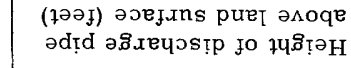 & & $\vdots$ & & $m$ & $\stackrel{p}{\sim}$ & & 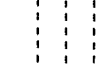 & 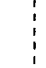 & $\vec{o}$ \\
\hline 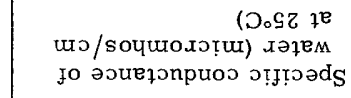 & & $\vdots$ & & 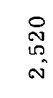 & $\begin{array}{l}\text { o } \\
\text { in } \\
\text { in }\end{array}$ & $\begin{array}{lll}1 & 1 \\
& 1 & 1 \\
& 1 & 1 \\
& 1 & 1 \\
1 & 1 & 1\end{array}$ & $\begin{array}{lll}10 \\
\text { in } \\
\text { in } \\
\vdots\end{array}$ & 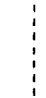 & 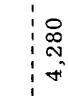 \\
\hline (ㅂ. & & 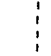 & & 운 & $\stackrel{N}{\sim}$ & i & \begin{tabular}{l}
$\infty$ \\
\hdashline
\end{tabular} & & i \\
\hline хәғем јо әs & 0202 & $\infty$ & z & en & a & $\pi a^{n} \cos n$ & z心口 & z & $n z$ \\
\hline xəMod jo $ә \mathrm{~d} \kappa_{\mathrm{I}}$ & 田3 & 3 & z & & 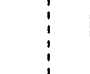 & 定国国国 & z & z & 红 \\
\hline 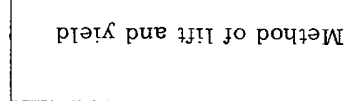 & 已े & $\vec{U}$ & 学 & 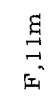 & 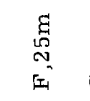 & $\vec{U} \triangleleft 4$ & 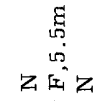 & z & \\
\hline 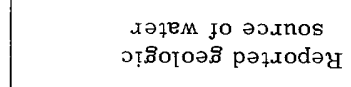 & $\vdots \vdots$ & 苟 & $\begin{array}{c}1 \\
\vdots \\
\vdots\end{array}$ & $\overrightarrow{0}$ & ชี่ & $\begin{array}{l:l}1 & -1 \\
\vdots & 0\end{array}$ & \begin{tabular}{c:c} 
& 0 \\
\hdashline & 1
\end{tabular} & $\vdots$ & $\begin{array}{l:}\vdots \\
\vdots \\
\vdots \\
\vdots \\
\vdots\end{array}$ \\
\hline 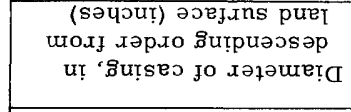 & 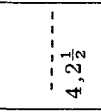 & $\vec{m}$ & $m$ & $\stackrel{\infty}{m}$ & $\sim$ & $\begin{array}{l:ll} & \\
m & \infty \\
& & \end{array}$ & $m \infty \stackrel{n}{n}$ & $m$ & $\tilde{n}^{N}$ \\
\hline 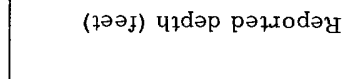 & 足 & 品 & 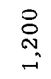 & $\underset{\infty}{\infty}$ & $\stackrel{8}{\stackrel{\circ}{\rightarrow}}$ & 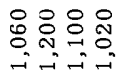 & 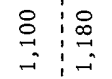 & 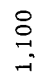 & 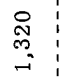 \\
\hline (рәдлоdә & 嵌怘 & $\begin{array}{l}0 \\
0 \\
0 \\
-1\end{array}$ & $\begin{array}{l}0 \\
0 \\
-1\end{array}$ & 苦 & 管 & 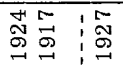 & 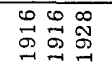 & $\underset{\mathcal{N}}{\stackrel{D}{\rightarrow}}$ & $\begin{array}{l:}\vec{b} \\
\stackrel{\vec{g}}{\rightarrow}:\end{array}$ \\
\hline 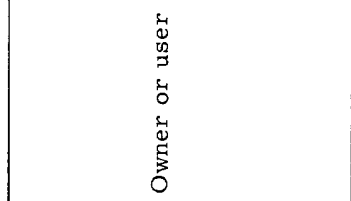 & 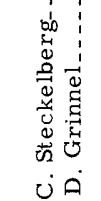 & $\begin{array}{l}\vdots \\
\vdots \\
\vdots \\
\Xi \\
\Xi \\
\Xi \\
0 \\
0 .\end{array}$ & 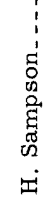 & $\begin{array}{l}\vdots \\
\vdots \\
\vdots \\
0 \\
0 \\
0 \\
02 \\
0 \\
\vdots \\
3 \\
3\end{array}$ & 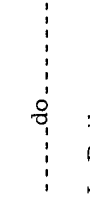 & 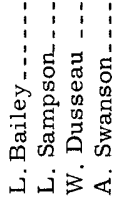 & 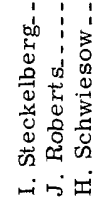 & $\begin{array}{l}\vdots \\
\vdots \\
0 \\
0 \\
0 \\
0 \\
0 \\
0 \\
0\end{array}$ & 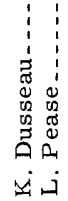 \\
\hline $\overrightarrow{0}$ & 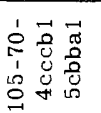 & $\begin{array}{l}\vec{u} \\
\frac{0}{0} \\
\text { مे }\end{array}$ & $\begin{array}{l}\overrightarrow{0} \\
0 \\
0 \\
0\end{array}$ & $\begin{array}{l}\overrightarrow{0} \\
\stackrel{0}{0} \\
\vec{Z}\end{array}$ & 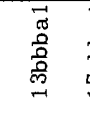 & 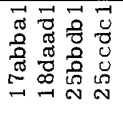 & 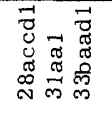 & 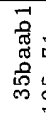 & $\begin{array}{l}\overrightarrow{0}: \overrightarrow{0} \\
00 \\
00 \\
0 \\
0 \\
0\end{array}$ \\
\hline
\end{tabular}




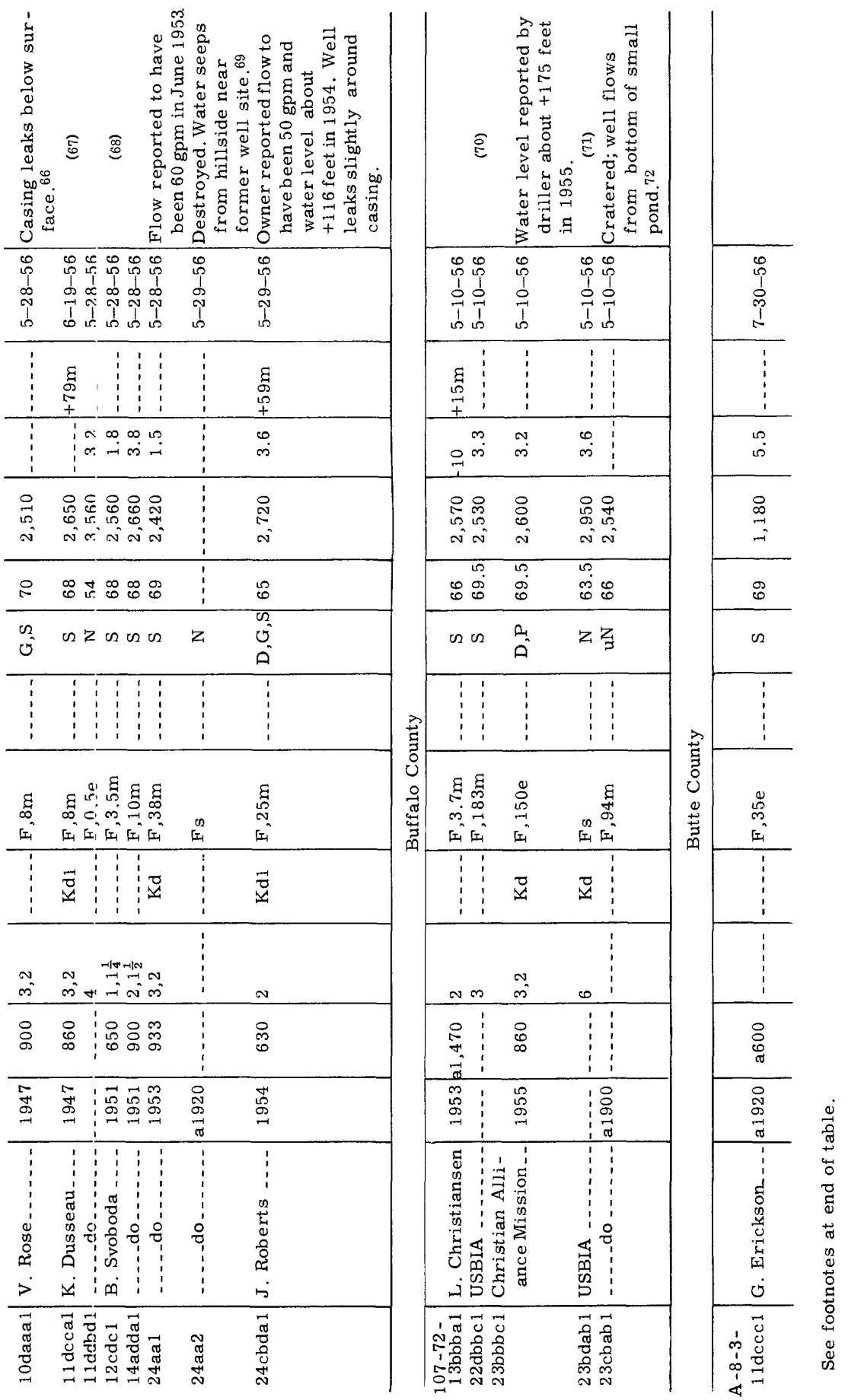




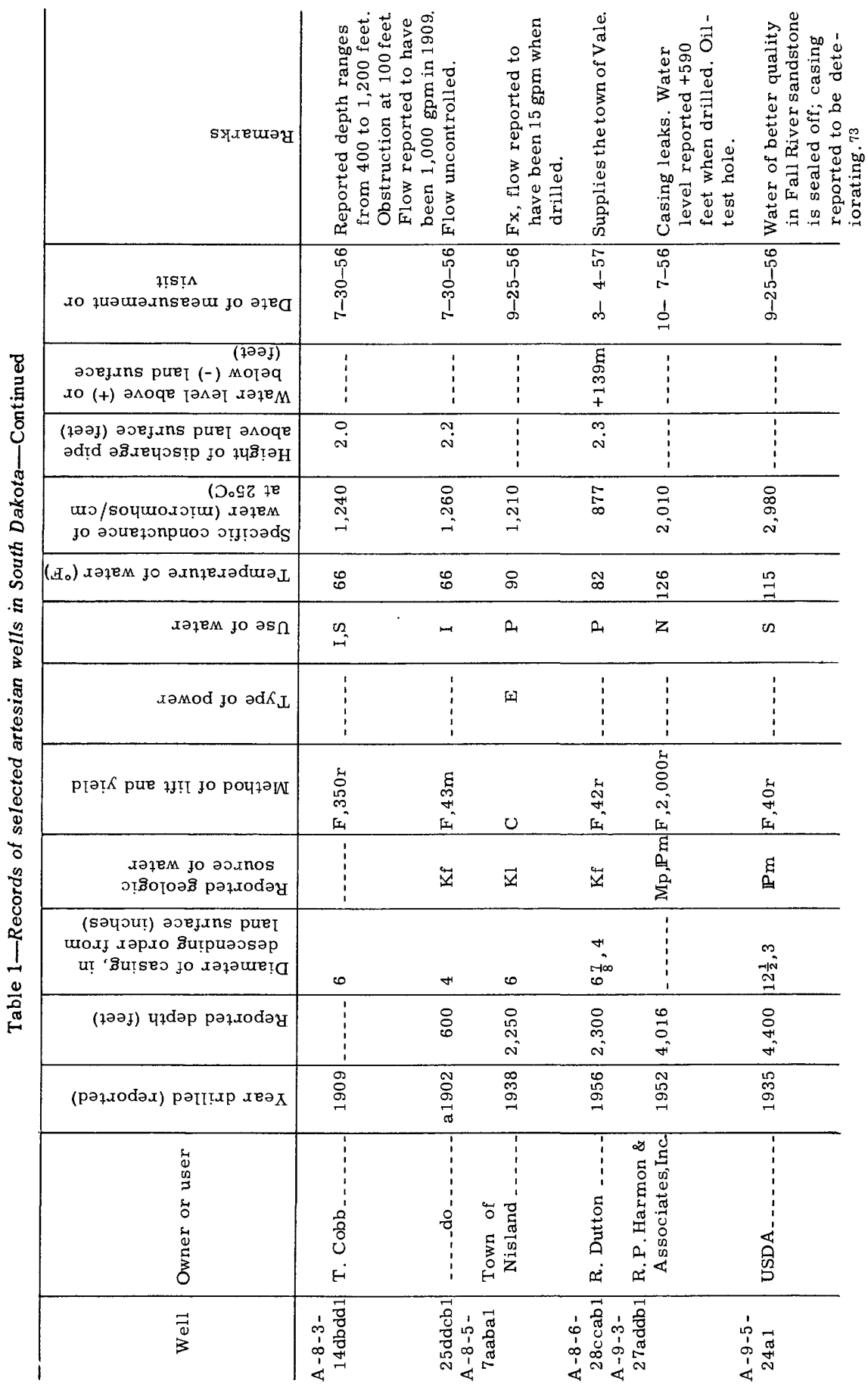




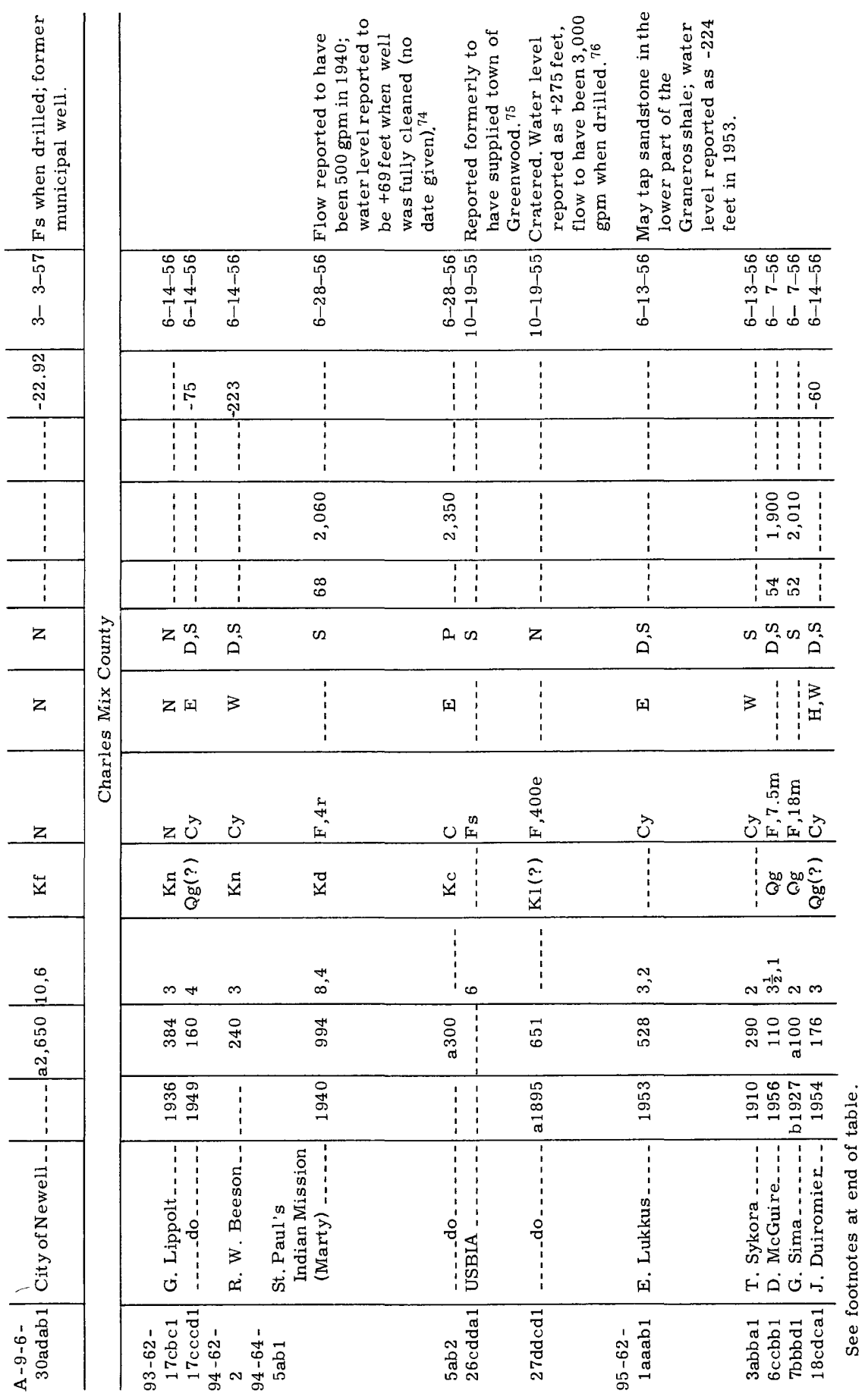




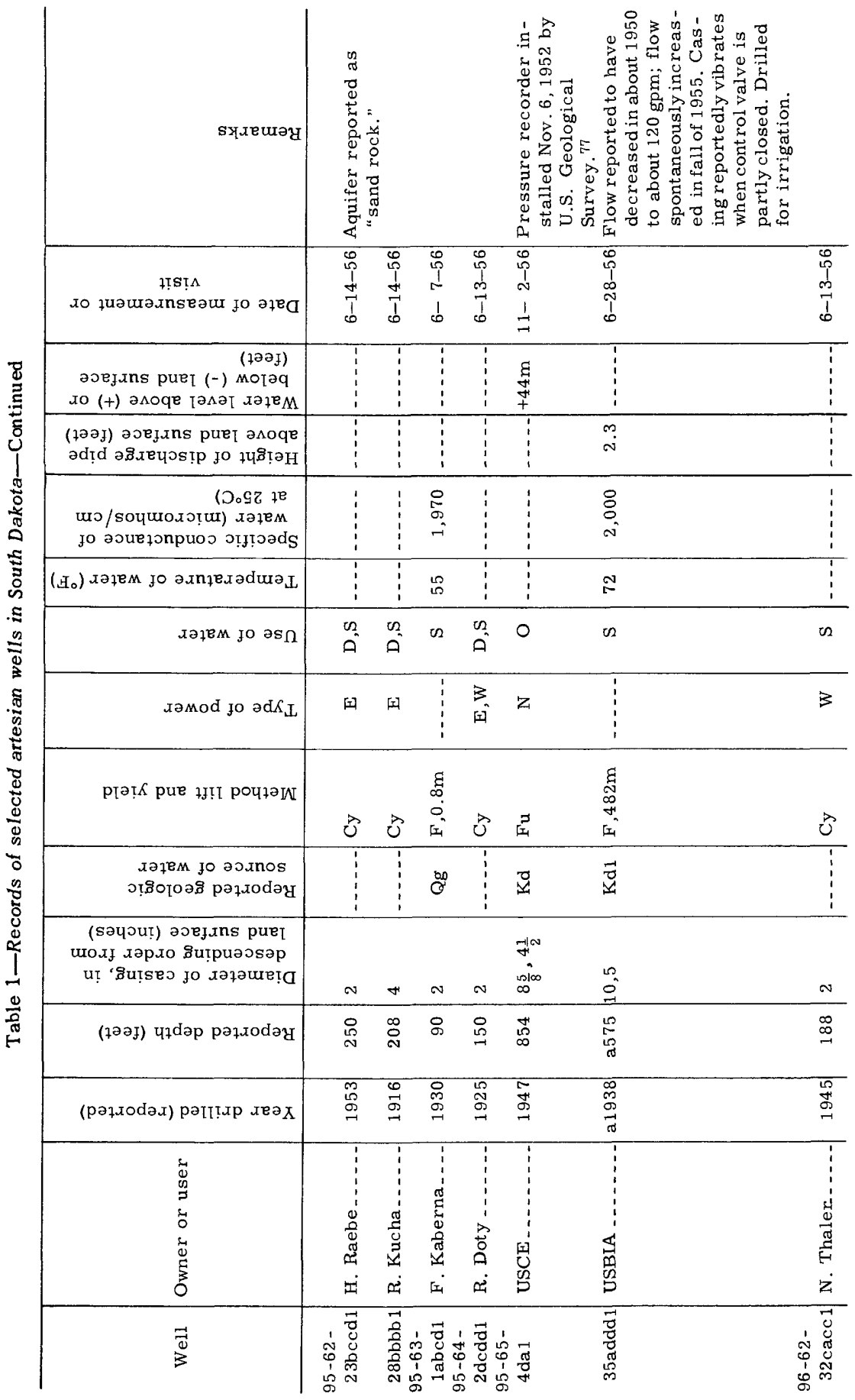




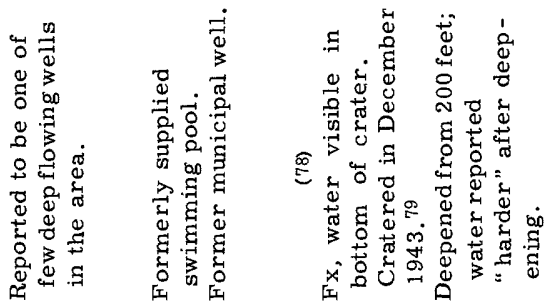

\begin{tabular}{|c|c|c|c|c|}
\hline 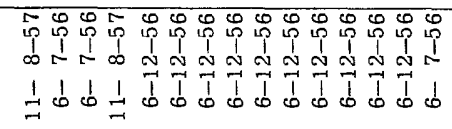 & $\begin{array}{lll}0 & 0 & 0 \\
0 & 0 & 0 \\
0 & 0 & 0 \\
1 & 1 & 1 \\
7 & m & 1 \\
1 & 1 & 1 \\
0 & 0 & 0\end{array}$ & $\begin{array}{ll}0 & 0 \\
0 & 0 \\
0 & 0 \\
1 & 0 \\
1 & 0 \\
1 & 0\end{array}$ & $\begin{array}{ll}0 & 0 \\
0 & 0 \\
1 & 1 \\
5 & 0 \\
1 & 1 \\
-1 & 0\end{array}$ & $\begin{array}{l}0 \\
0 \\
1 \\
1 \\
1\end{array}$ \\
\hline 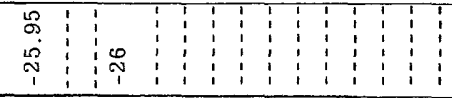 & $\begin{array}{l}8 \\
\infty \\
17 \\
1\end{array}$ & : & & 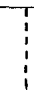 \\
\hline 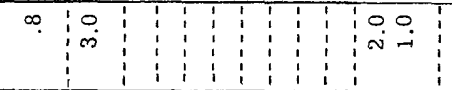 & & $\begin{array}{c}T \\
\vdots \\
1\end{array}$ & $\begin{array}{l}\infty \\
\dot{i}\end{array}$ & : \\
\hline 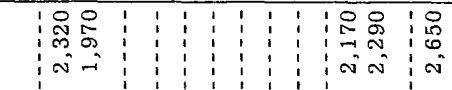 & & 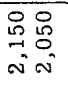 & 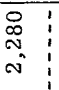 & $\begin{array}{l}8 \\
\text { th } \\
\text { in }\end{array}$ \\
\hline 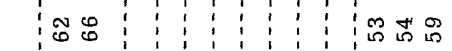 & טิ & กี้ ถึ & $\therefore$ & 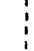 \\
\hline 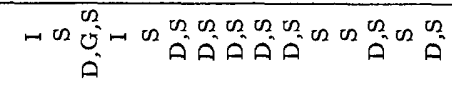 & $a^{2} a^{2}, z$ & 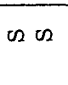 & 小 & D \\
\hline : & 国 3 & & : & [19 \\
\hline 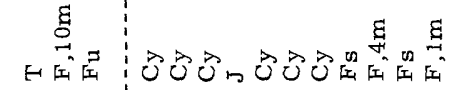 & 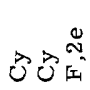 & 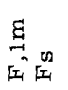 & 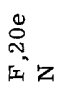 & $\hat{U}$ \\
\hline 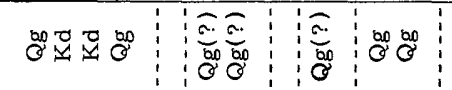 & : & 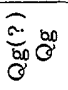 & चै & $\stackrel{\infty}{\sigma}^{\infty}$ \\
\hline
\end{tabular}

\begin{tabular}{|c|c|c|c|c|}
\hline$\stackrel{-1 N}{\infty} \sim$ & $\sim \sim 0$ & $+N$ & $\infty_{\infty}^{\infty}$ & $\stackrel{n}{\rightarrow-1}$ \\
\hline 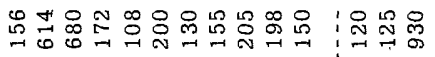 & : & i̊ & $\begin{array}{c}N \\
\infty \\
\infty\end{array}$ & $\overrightarrow{0}$ \\
\hline
\end{tabular}

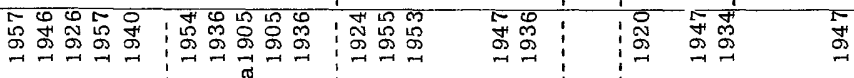

\begin{tabular}{|c|c|c|c|c|}
\hline 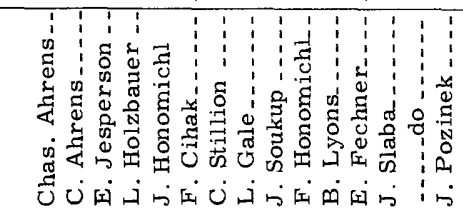 & 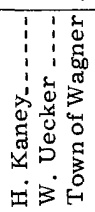 & 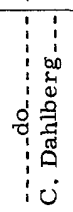 & 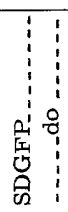 & 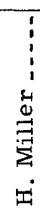 \\
\hline 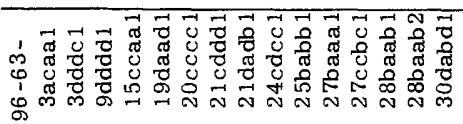 & 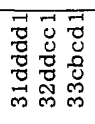 & 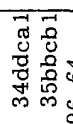 & 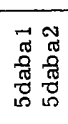 & $\begin{array}{l}\overrightarrow{7} \\
\tilde{J} \\
0 \\
0\end{array}$ \\
\hline
\end{tabular}




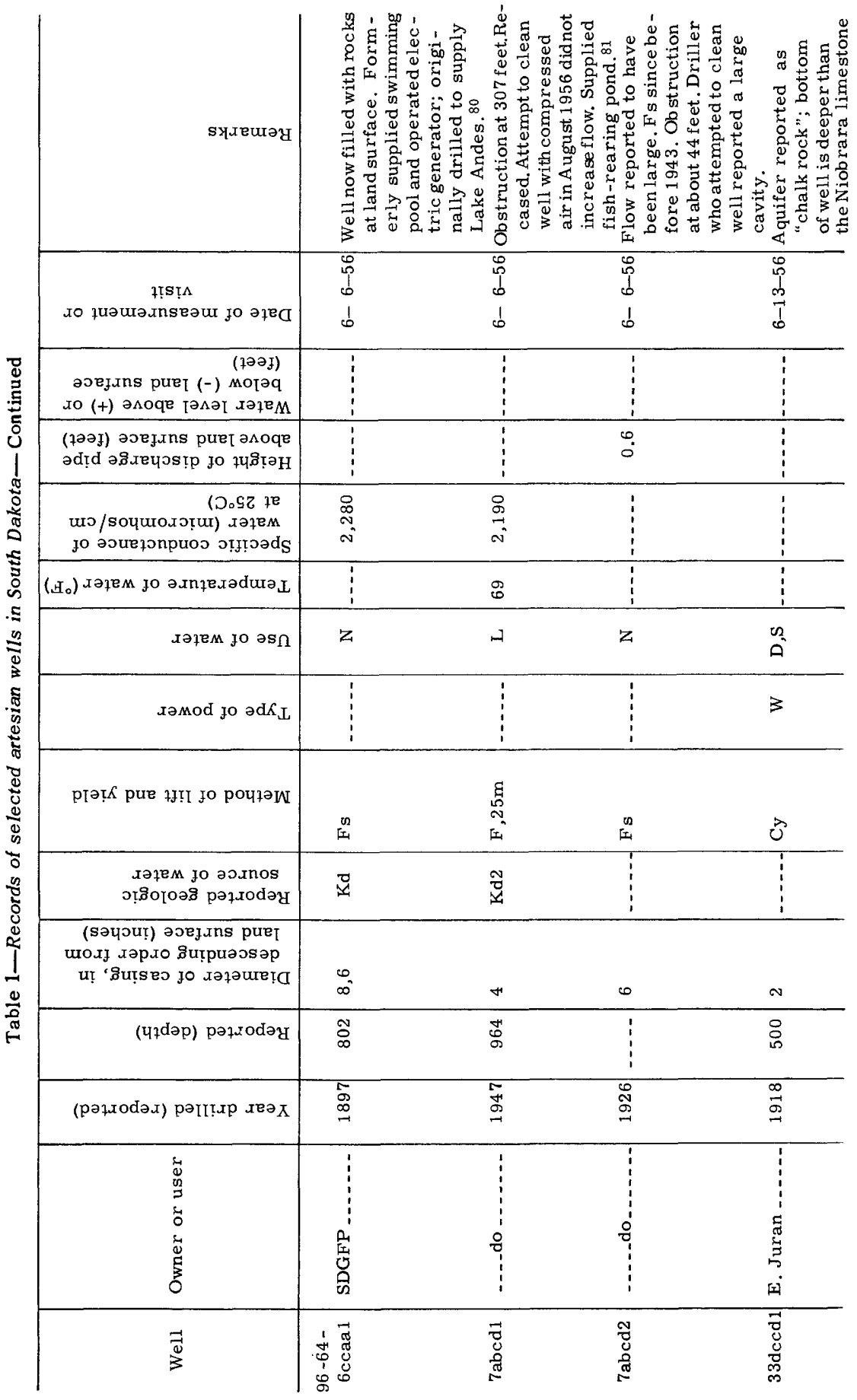




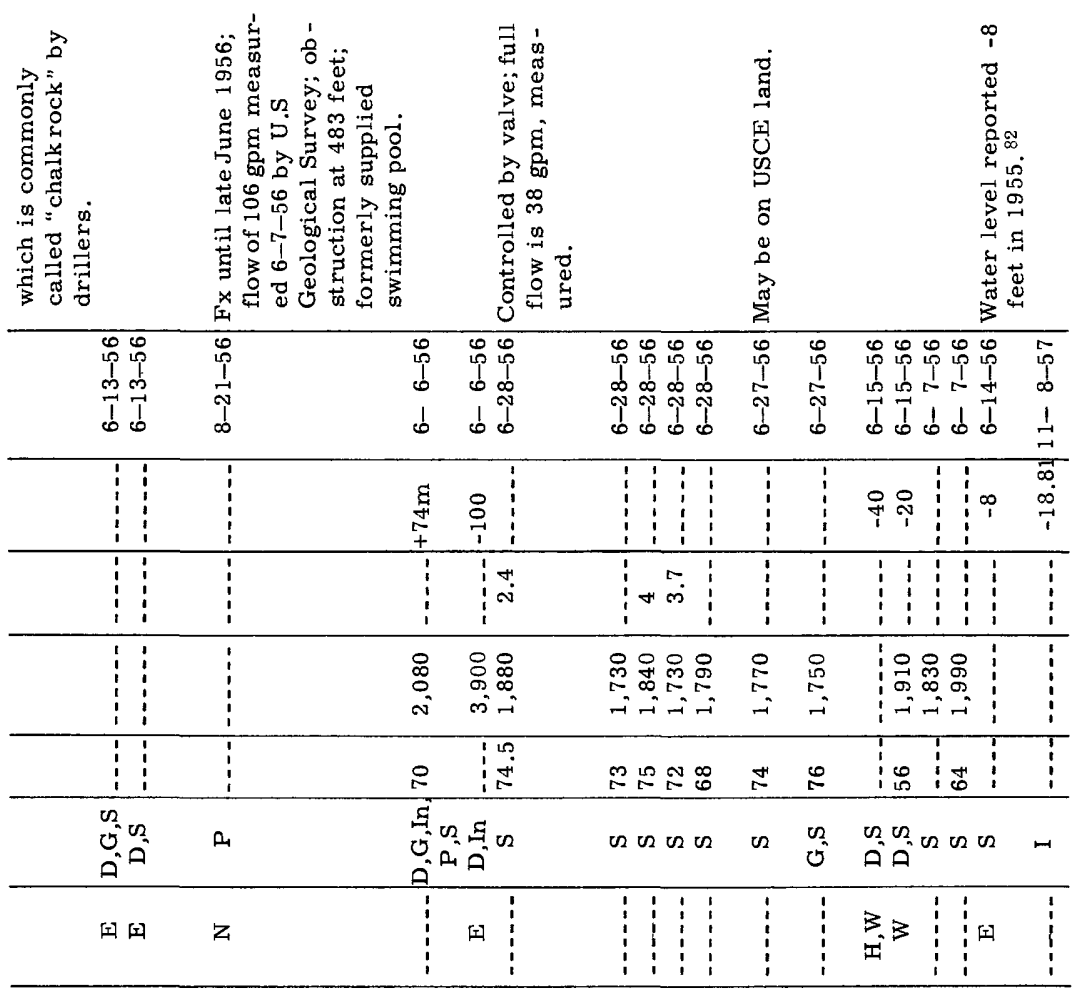

\begin{tabular}{|c|c|c|c|c|c|c|c|c|}
\hline ठें & z & 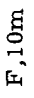 & $\begin{array}{r}\mathscr{g} \\
\qquad \square\end{array}$ & 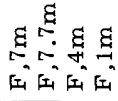 & 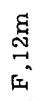 & $\begin{array}{l}\text { g్ } \\
\text { N. } \\
\text { \&్ }\end{array}$ & 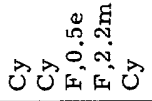 & $H$ \\
\hline$\sqrt{x}$ & i & $\begin{array}{l}3 \\
\vdots \\
\vdots\end{array}$ & 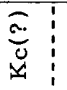 & & 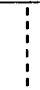 & $\bar{y}$ & 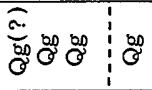 & $\stackrel{\infty}{\sigma}$ \\
\hline N & $\vdots$ & m & $\sim m$ & 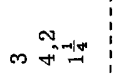 & N & $\frac{-100}{\infty}$ & $\sim \sim \sim \sim \sim$ & $\infty$ \\
\hline$\stackrel{\circ}{\stackrel{\circ}{\circ}} \underset{\sigma}{\stackrel{\infty}{\sigma}}$ & : & 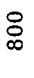 & 品早 & 용 융 & 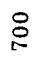 & 电 & 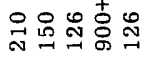 & in \\
\hline
\end{tabular}

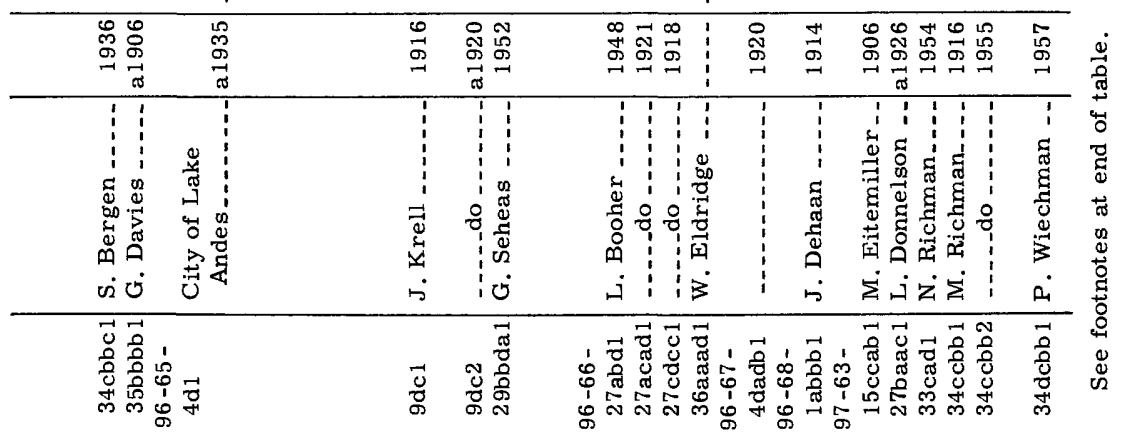




\begin{tabular}{|c|c|c|c|c|c|c|}
\hline syฺxeuəy & 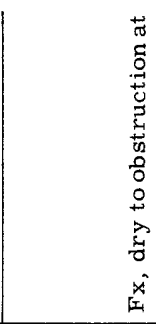 & & & & 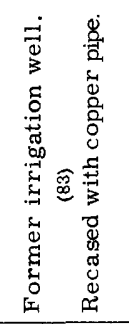 & \\
\hline 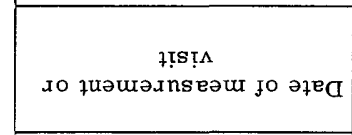 & $\begin{array}{lll}5 & 0 & 0 \\
0 & 0 & 0 \\
1 & 1 & 1 \\
0 & 1 & 1 \\
1 & 1 & 1 \\
7 & 0 & 0 \\
\end{array}$ & $\begin{array}{ll}0 & 0 \\
0 & 0 \\
1 & 0 \\
1 & 0 \\
1 & 1 \\
0 & 0\end{array}$ & $\begin{array}{l}0 \\
0 \\
1 \\
0 \\
1 \\
0\end{array}$ & \begin{tabular}{l}
0 \\
$i$ \\
1 \\
\multirow{1}{1}{} \\
0
\end{tabular} & $\begin{array}{llll}0 & 0 & 0 & 0 \\
0 & 0 & 0 & 0 \\
1 & 1 & 1 & 1 \\
0 & 0 & 0 & 5 \\
1 & 1 & 1 & 1 \\
0 & 0 & 0 & 0\end{array}$ & $\begin{array}{lll}0 & 0 & 0 \\
0 & 0 & 5 \\
1 & 1 & 1 \\
= & 7 & 0 \\
1 & 1 & 1 \\
0 & 0 & c\end{array}$ \\
\hline 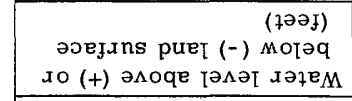 & 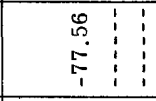 & 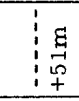 & $\begin{array}{c}\vdots \\
\vdots \\
\vdots \\
\end{array}$ & $\vdots$ & $\begin{array}{r:}1 \\
1 \\
1\end{array}$ & $\begin{array}{r:}\vdots \\
\vdots \\
\vdots \\
\vdots \\
1 \\
\end{array}$ \\
\hline 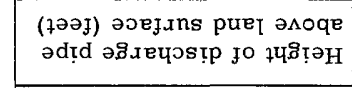 & 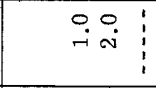 & 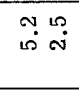 & $\stackrel{\infty}{\sim}$ & $\vdots$ & 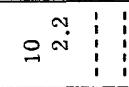 & 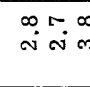 \\
\hline 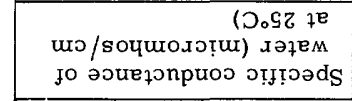 & 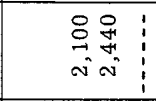 & 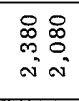 & 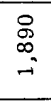 & $\begin{array}{l}8 \\
\stackrel{8}{0} \\
\text { i }\end{array}$ & 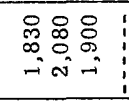 & 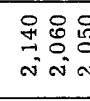 \\
\hline 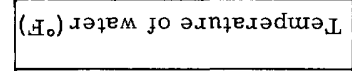 & $\begin{array}{l}1 \\
0 \\
0\end{array}$ & $\ddot{0}$ & 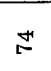 & \& & 象 & 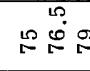 \\
\hline хәңем јо әs $\Omega$ & ッ片 & $n \pi$ & $\sigma$ & n & $\pi n \pi n$ & $\cos 20$ \\
\hline 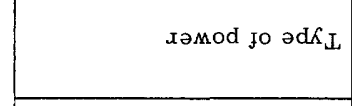 & $0:$\begin{tabular}{l:c} 
\\
\hdashline \\
\hdashline
\end{tabular} & $\vdots$ & $\begin{array}{c}\vdots \\
\vdots \\
\vdots \\
\vdots\end{array}$ & $\vdots$ & 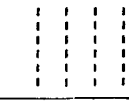 & $\begin{array}{l:c}\vdots \\
\vdots \\
\vdots \\
\vdots \\
\end{array}$ \\
\hline 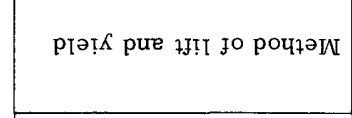 & 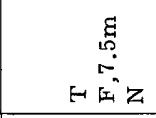 & 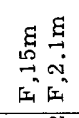 & $\begin{array}{l}\tilde{g} \\
0 \\
\vdots\end{array}$ & 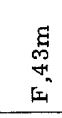 & 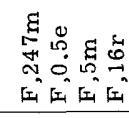 & 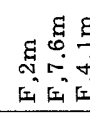 \\
\hline 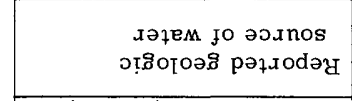 & $\begin{array}{l:c}\infty & \vdots \\
\sigma^{\infty} & \vdots \\
& \vdots\end{array}$ & : & $\vdots$ & ขै & 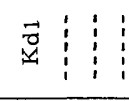 & $:$ \\
\hline 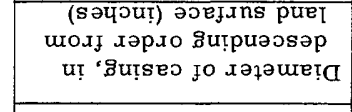 & $\underset{\rightarrow-1}{\infty} \infty$ & a & $\underset{\tilde{\sigma}}{\pi}$ & N & $\infty \sim \stackrel{\sim}{n}$ & 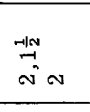 \\
\hline (ұәәу) чұdәр рәұлоdәу & 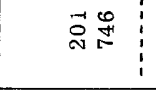 & 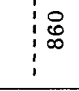 & 足 & ஜீ & $\begin{array}{l:l}0 & 0 \\
0 & 8 \\
& 0 \\
\end{array}$ & $\begin{array}{ll}1 \\
1 \\
1 & 1 \\
1 & 1 \\
\end{array}$ \\
\hline 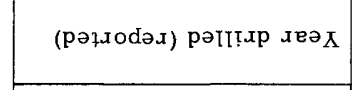 & 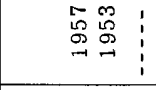 & 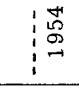 & $\begin{array}{l}8 \\
\infty \\
\infty \\
\pi \\
\end{array}$ & 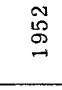 & 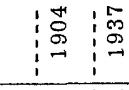 & $\begin{array}{l:}\vdots \\
\vdots \\
\vdots \\
\end{array}$ \\
\hline 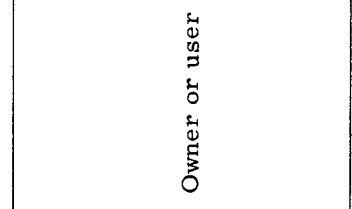 & 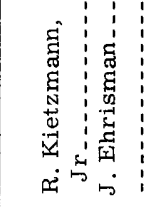 & 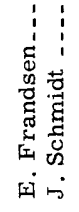 & $\begin{array}{l}\vdots \\
\vdots \\
\vdots \\
0 \\
0 \\
0 \\
\vdots\end{array}$ & 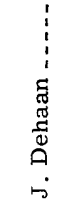 & 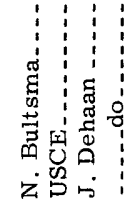 & 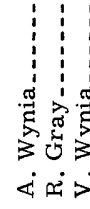 \\
\hline$\frac{\overline{0}}{3}$ & 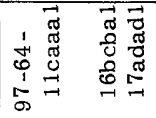 & $\begin{array}{l}\overrightarrow{0} \\
\overrightarrow{0} \\
00 \\
0 \\
0 \\
\overrightarrow{0}\end{array}$ & 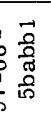 & 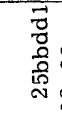 & 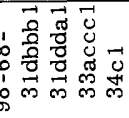 & 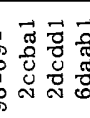 \\
\hline
\end{tabular}




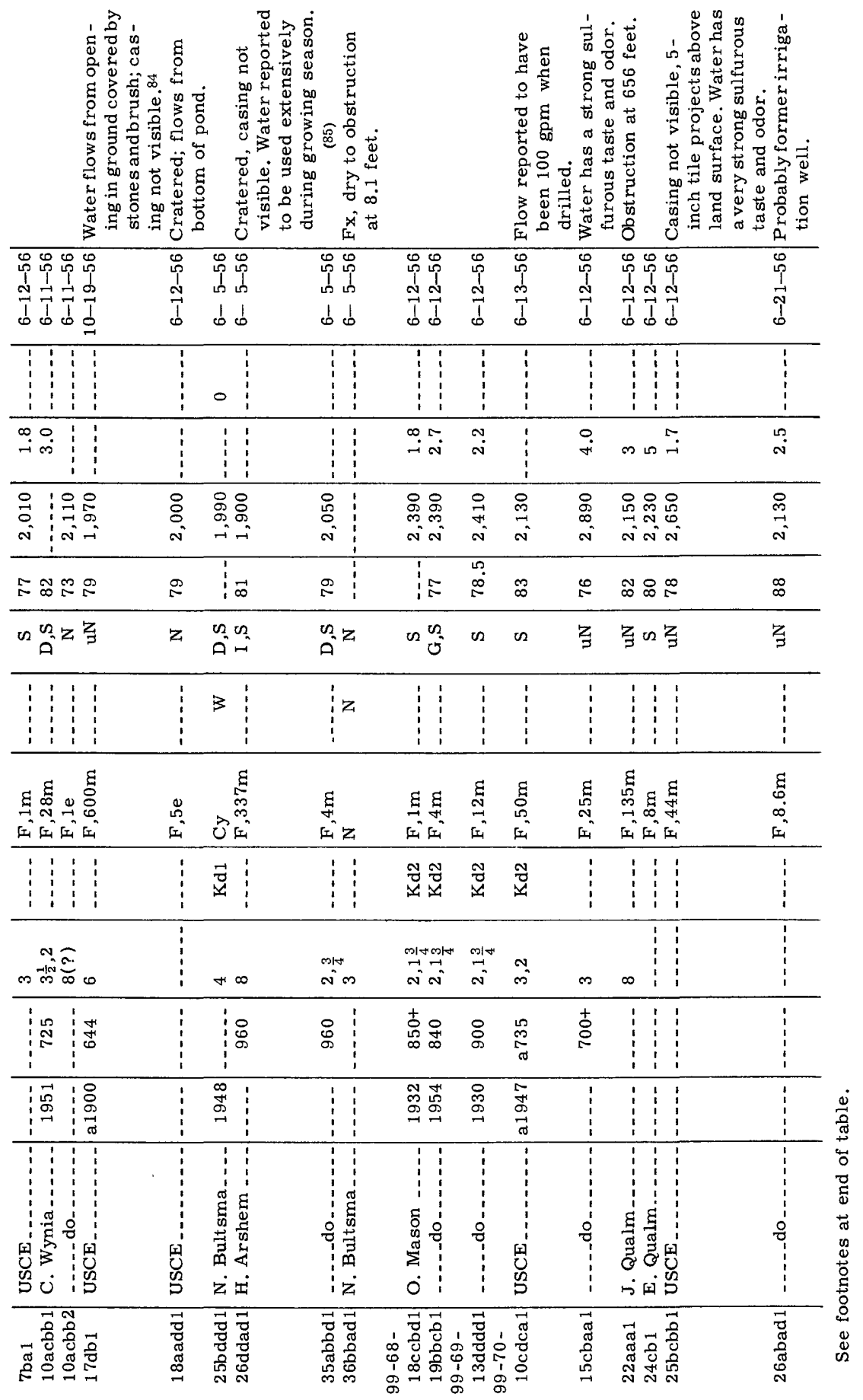




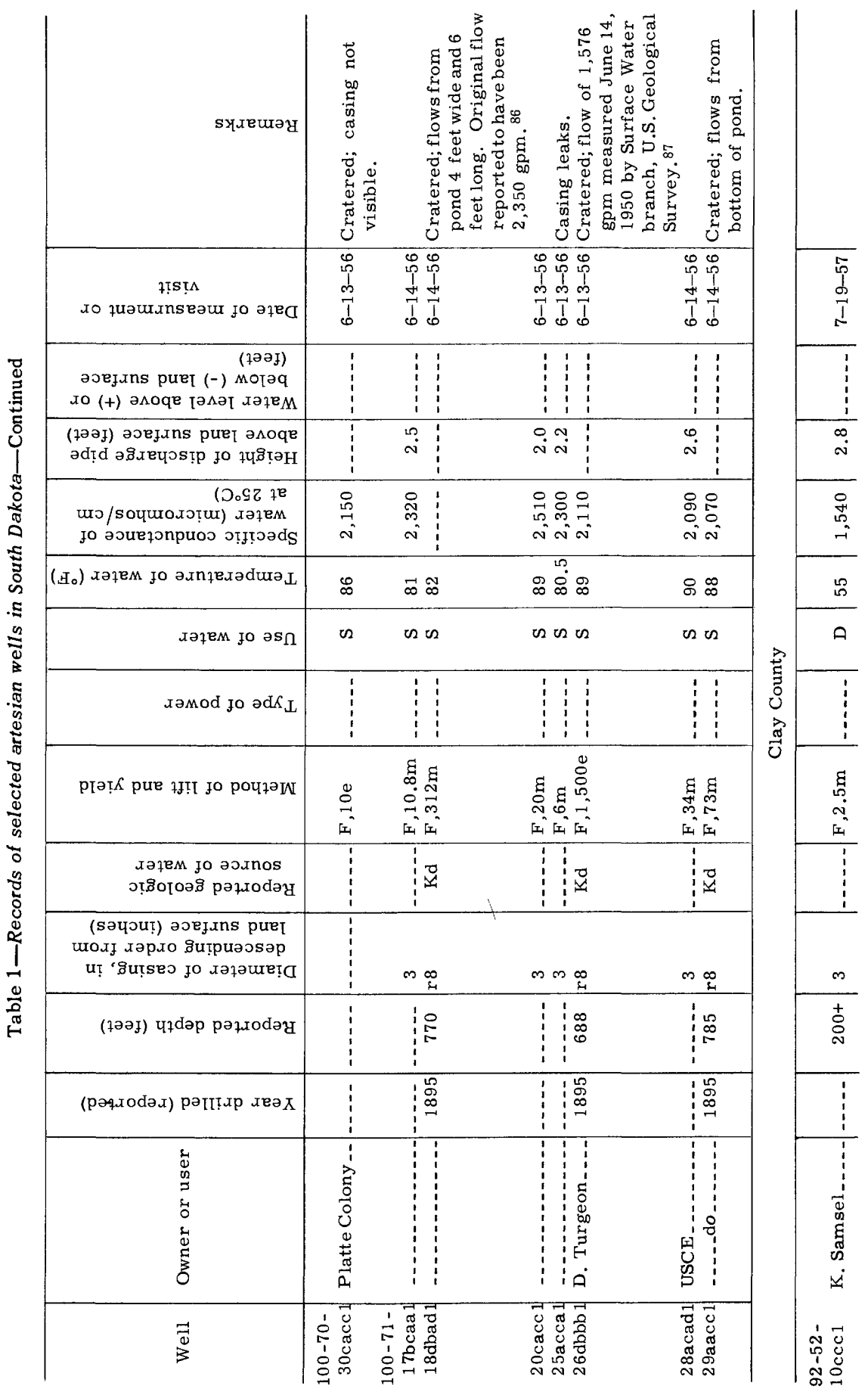




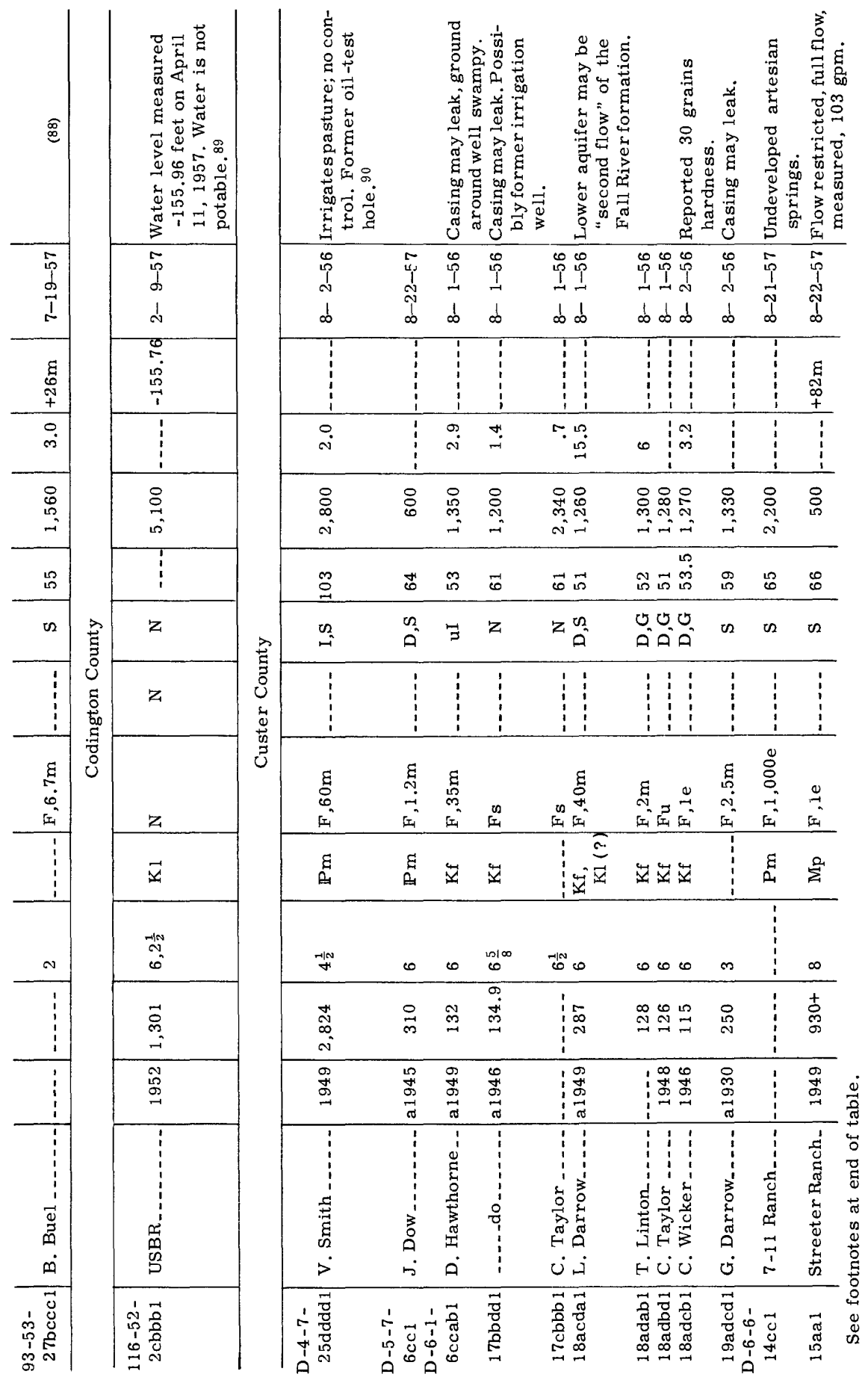




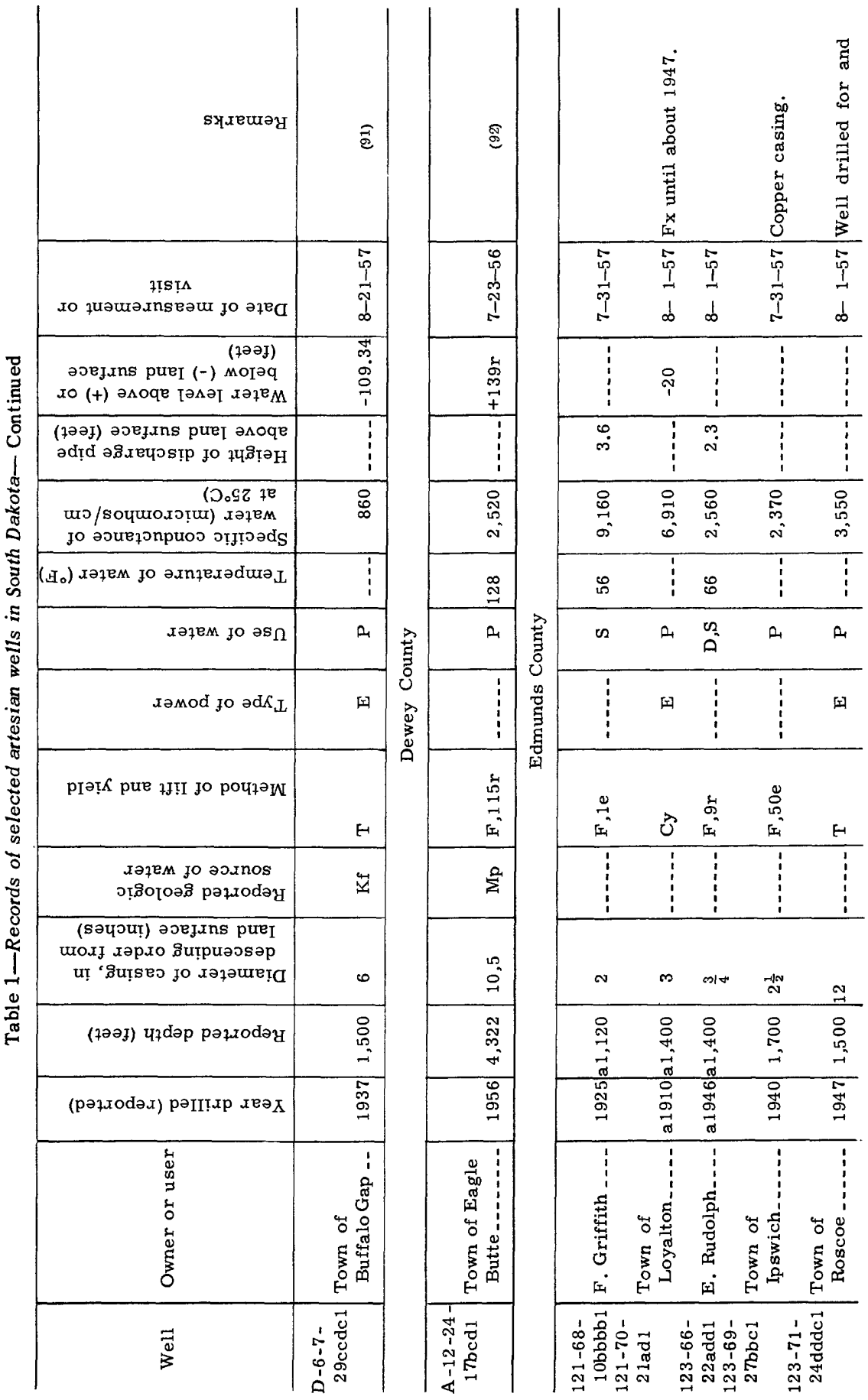




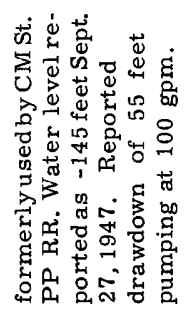

\begin{tabular}{|c|c|c|c|c|c|}
\hline 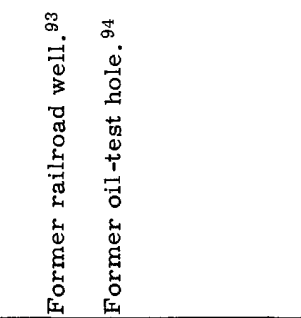 & 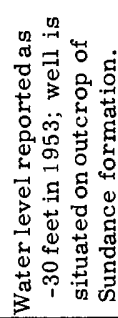 & 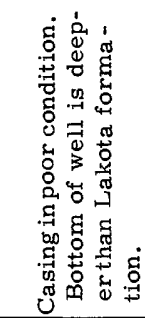 & 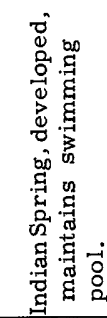 & & 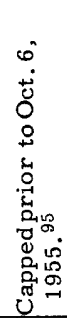 \\
\hline 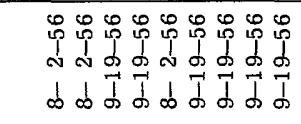 & 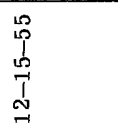 & $\begin{array}{lll}0 & 0 \\
0 & 0 \\
1 & 1 \\
N & 0 \\
1 & 1 \\
0 & \infty\end{array}$ & $\begin{array}{l}\infty \\
1 \\
1 \\
1 \\
m\end{array}$ & & $\begin{array}{l}\stackrel{4}{0} \\
\stackrel{1}{0} \\
\infty\end{array}$ \\
\hline 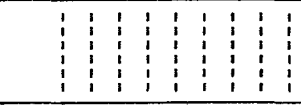 & 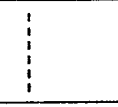 & $\begin{array}{l:}\vdots \\
\vdots \\
\vdots \\
\end{array}$ & 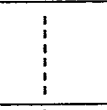 & & 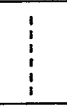 \\
\hline 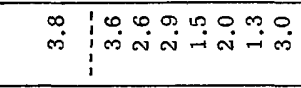 & 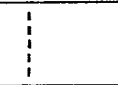 & $7 \stackrel{\circ}{-1}$ & $\vdots$ & & $\stackrel{5}{m}$ \\
\hline 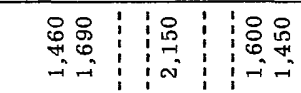 & $\begin{array}{l}\text { 오 } \\
\text { Hin } \\
\text { i }\end{array}$ & 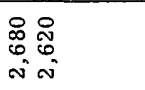 & $\begin{array}{l}\text { : } \\
\text { o. } \\
i\end{array}$ & & 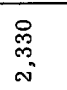 \\
\hline ํำ ถั & $\stackrel{\infty}{\forall}$ & 员 & $\stackrel{\infty}{\infty}$ & & $\stackrel{\infty}{\infty}$ \\
\hline 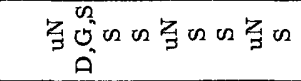 & $a^{n}$ & $a^{02}$ & $\Xi$ & & 乙 \\
\hline 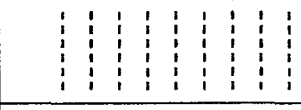 & 되 & 되 & $\begin{array}{c}\vdots \\
\vdots \\
\vdots\end{array}$ & & $\begin{array}{c}\vdots \\
\vdots \\
\vdots \\
\end{array}$ \\
\hline 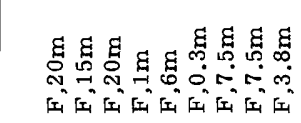 & $u$ & 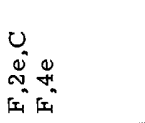 & 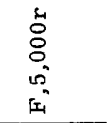 & & $\begin{array}{l}\stackrel{D}{0}^{\circ} \\
\text { in } \\
\text { 圷 }\end{array}$ \\
\hline 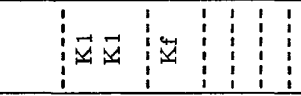 & $\stackrel{n}{2}$ & $\vec{y}$ & ڤ્વ & & $\underset{\mu}{q}$ \\
\hline 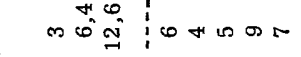 & $\varphi$ & $\sim \infty$ & 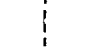 & & 오 \\
\hline 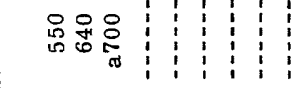 & $\stackrel{?}{\longleftarrow}$ & 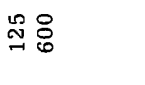 & 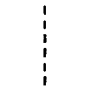 & & $\underset{\sim}{\mathbb{N}}$ \\
\hline 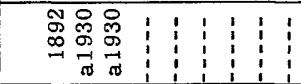 & $\begin{array}{l}0 \\
0 \\
0 \\
0 \\
-1\end{array}$ & 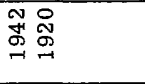 & $\vdots$ & & 藟 \\
\hline 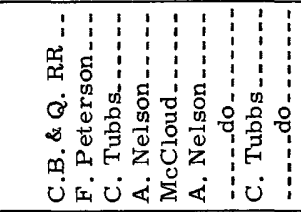 & 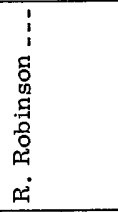 & 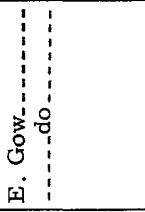 & 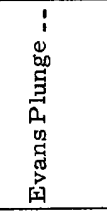 & \multicolumn{2}{|c|}{ 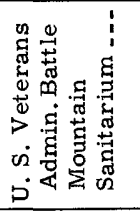 } \\
\hline 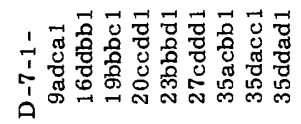 & 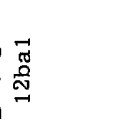 & 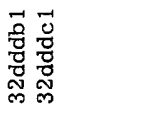 & 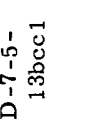 & 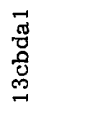 & \\
\hline
\end{tabular}




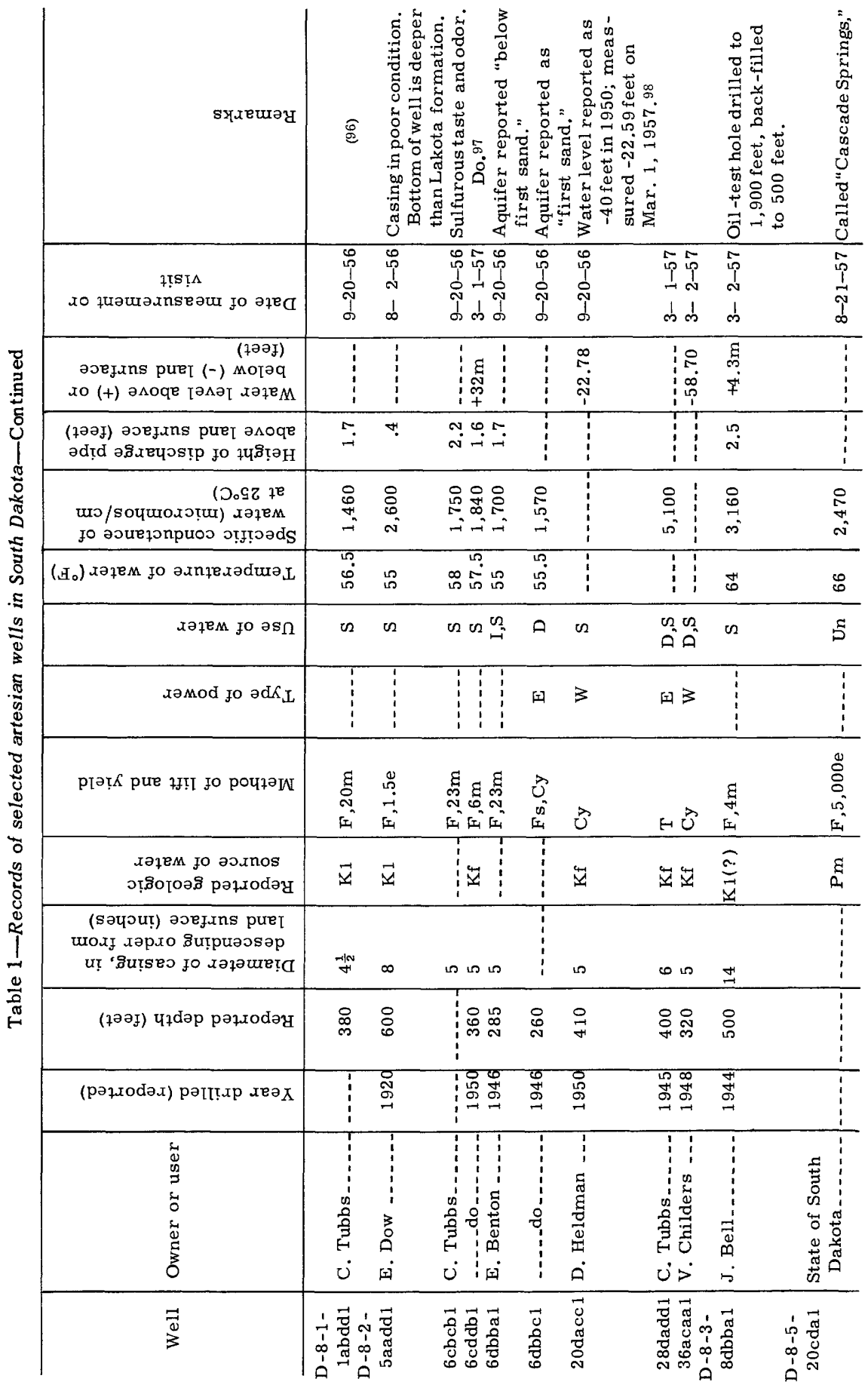



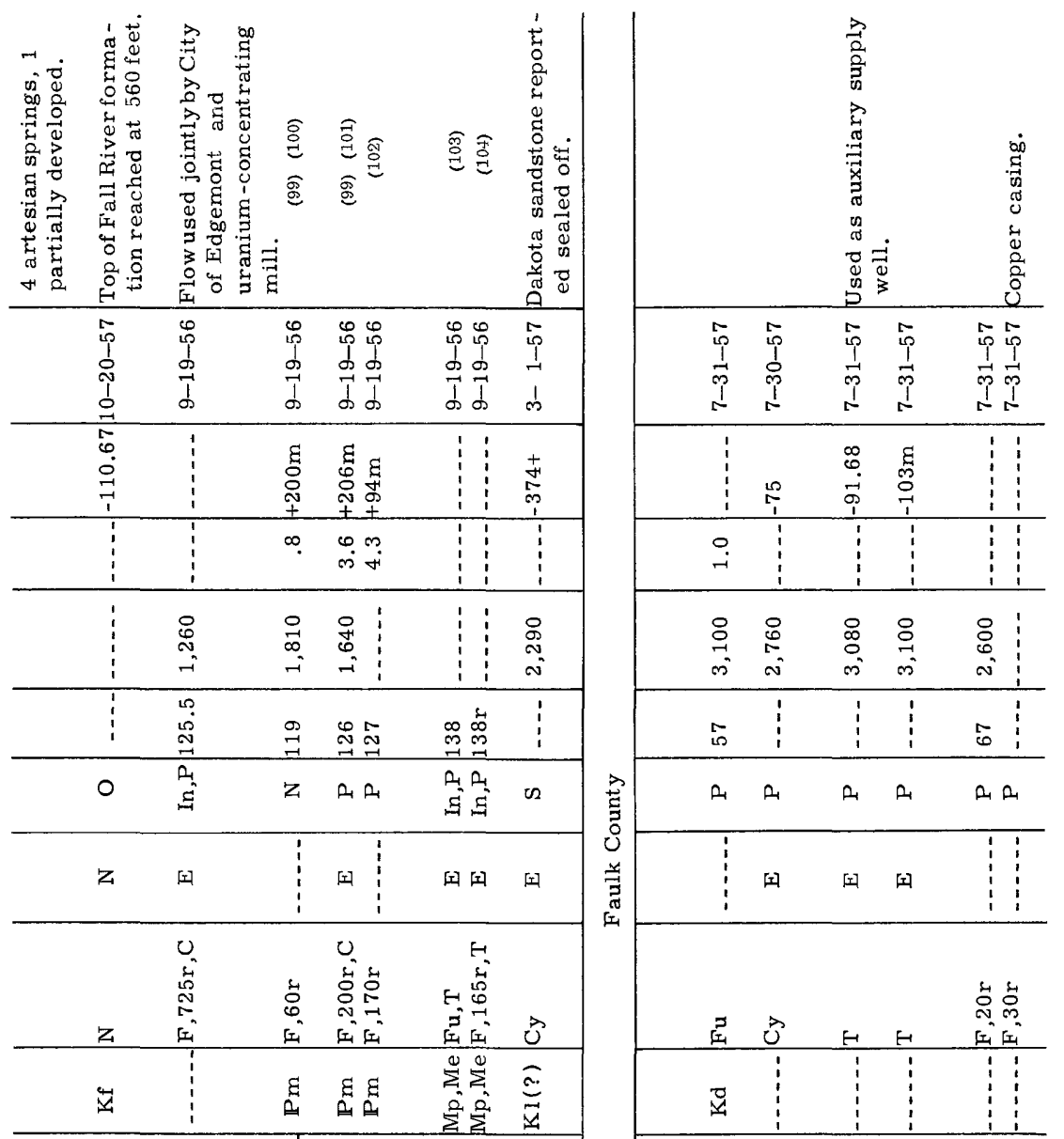

\begin{tabular}{|c|c|c|c|c|}
\hline z & 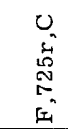 & 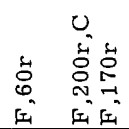 & 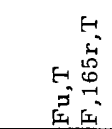 & $\vec{U}$ \\
\hline 荘 & 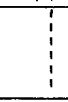 & $\begin{array}{c}\xi \\
\Xi\end{array}$ & $\begin{array}{l}\sum_{1}^{0} \sum_{1}^{0} \\
\sum_{1}^{2} \sum_{i}^{0}\end{array}$ & 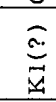 \\
\hline 0 & 0 & 0 & 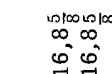 & 0 \\
\hline ల్ & $\begin{array}{l}10 \\
\stackrel{5}{\circ} \\
\text { N }\end{array}$ & $\begin{array}{lll}\circ & m & \infty \\
\infty & \infty & \infty \\
\infty & \infty & -1 \\
\sim & N & \infty\end{array}$ & $\begin{array}{l}0 \vec{~} \\
\text { के } \\
\text { के } \\
\text { के }\end{array}$ & @ \\
\hline $\begin{array}{l}\text { 옹 } \\
\text { \& } \\
\text { - }\end{array}$ & \begin{tabular}{l}
0 \\
\multirow{\sigma}{*}{}
\end{tabular} & 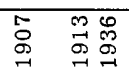 & 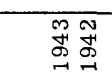 & $\begin{array}{l}0 \\
\stackrel{0}{\Xi} \\
\stackrel{\sigma}{\sigma}\end{array}$ \\
\hline 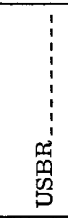 & 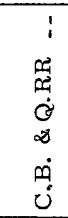 & 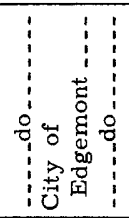 & 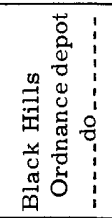 & 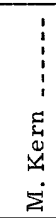 \\
\hline $\begin{array}{ll} & \\
1 & 0 \\
0 & 0 \\
1 & 0 \\
0 & 0 \\
1 & 0 \\
0 & \end{array}$ & 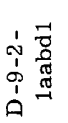 & 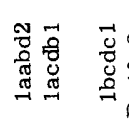 & 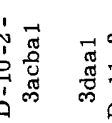 & 臯 \\
\hline
\end{tabular}

\begin{tabular}{|c|c|c|c|c|c|}
\hline$m$ & H & $\ddot{0}$ & $\begin{array}{l}\infty \\
\stackrel{0}{0}\end{array}$ & & $\stackrel{-1 N}{\sim}$ \\
\hline $\begin{array}{l}\stackrel{8}{\circ} \\
-\overrightarrow{0} \\
-1\end{array}$ & 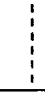 & $\begin{array}{l}\stackrel{\leftrightarrow}{0} \\
\text { N } \\
-\end{array}$ & 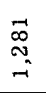 & & 品 \\
\hline & 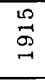 & $\begin{array}{l}\text { की } \\
\text { J }\end{array}$ & $\begin{array}{l}\text { 范 } \\
\text { 品 }\end{array}$ & & \begin{tabular}{l}
$\infty$ \\
\multirow{\sigma}{*}{} \\
$\stackrel{\sigma}{\sigma}$
\end{tabular} \\
\hline 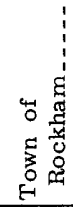 & 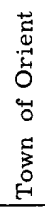 & 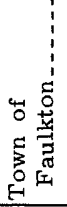 & $\begin{array}{l}1 \\
\vdots \\
\vdots \\
1 \\
1 \\
1 \\
1 \\
1\end{array}$ & 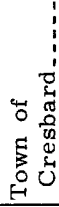 & 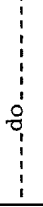 \\
\hline 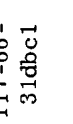 & $\begin{array}{l}8 \\
8 \\
0\end{array}$ & & 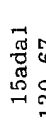 & $\begin{array}{l}\overrightarrow{0} \\
0 \\
\frac{0}{\pi} \\
\text { m }\end{array}$ & $\begin{array}{l}0 \\
0 \\
0 \\
\frac{0}{\pi} \\
-\infty \\
\infty\end{array}$ \\
\hline
\end{tabular}




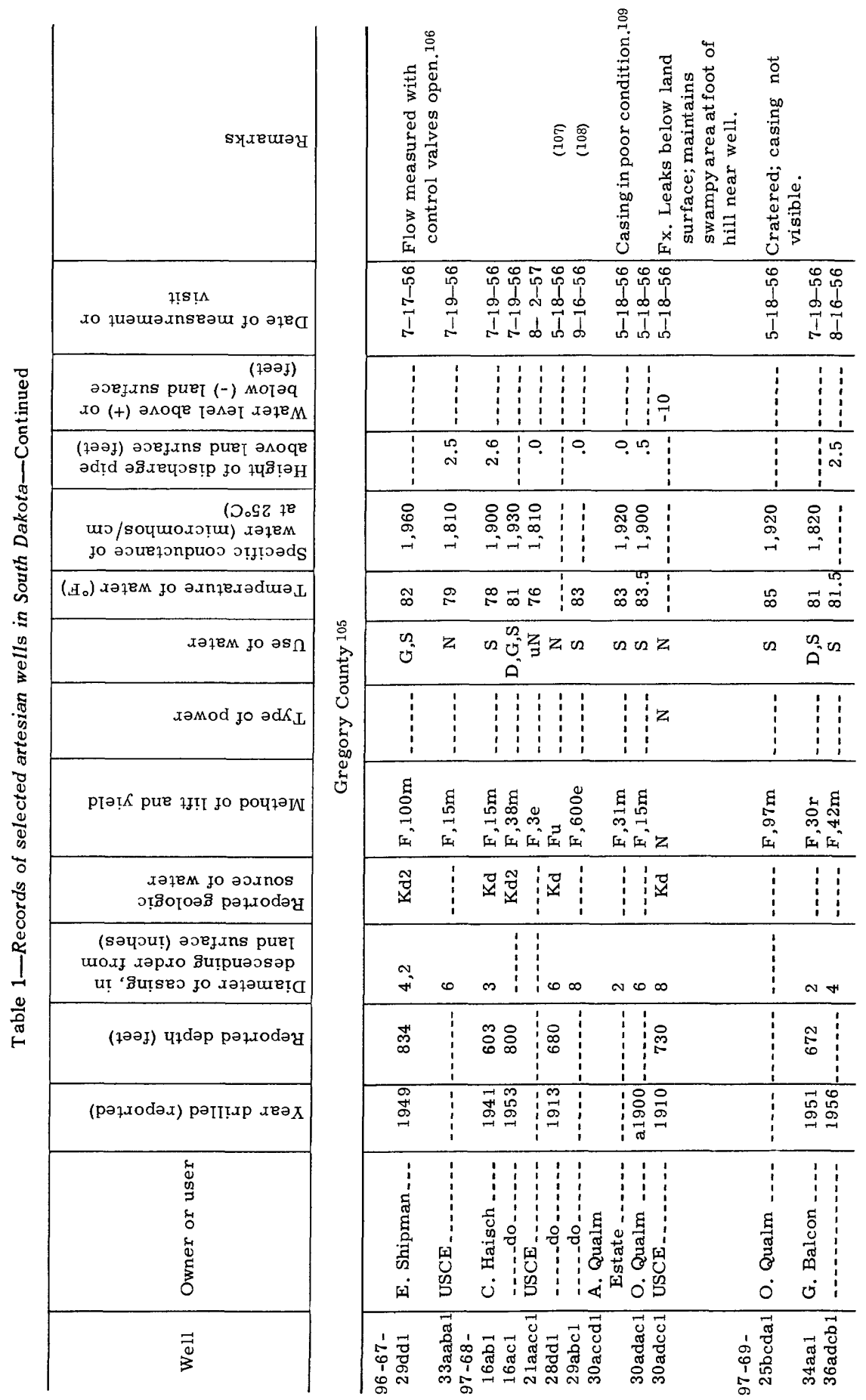




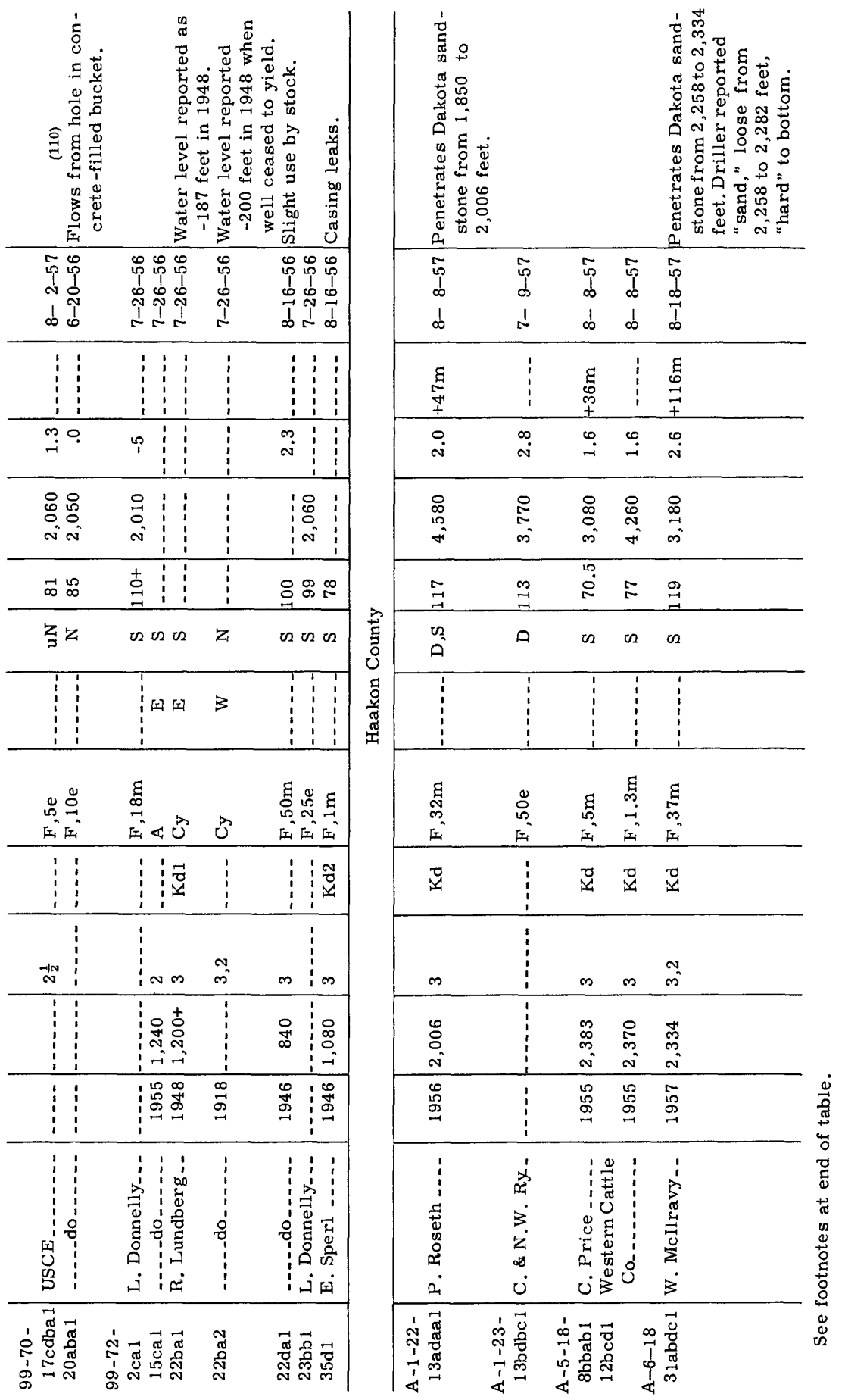




\begin{tabular}{|c|c|c|c|c|c|c|}
\hline sң.reurəy & 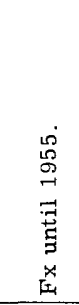 & 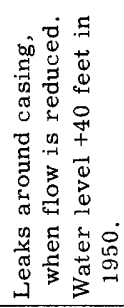 & & 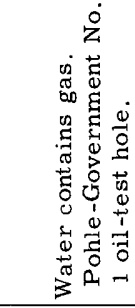 & 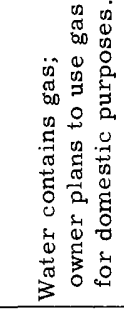 & 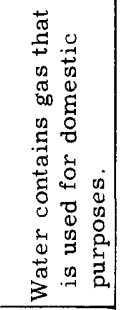 \\
\hline 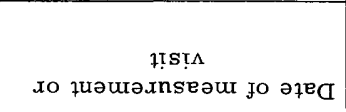 & $\begin{array}{l}i \\
i \\
i \\
i\end{array}$ & $\begin{array}{cc}i & 5 \\
1 & 1 \\
0 & 1 \\
1 & 1\end{array}$ & $\begin{array}{ll}5 & 5 \\
1 & 1 \\
0 & 1 \\
1 & 1 \\
1 & 1\end{array}$ & $\begin{array}{l}5 \\
0 \\
\infty \\
1\end{array}$ & $\begin{array}{l}5 \\
0 \\
0 \\
1 \\
1\end{array}$ & $\begin{array}{l}5 \\
1 \\
0 \\
1 \\
1\end{array}$ \\
\hline 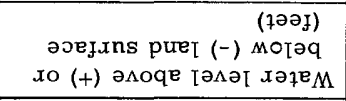 & $\begin{array}{l}\infty \\
0 \\
0 \\
0 \\
1 \\
1\end{array}$ & $\vdots$ & $\stackrel{\infty}{\not}$ & 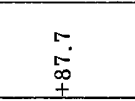 & $\vdots$ & $\begin{array}{c}\vdots \\
\vdots \\
\vdots \\
\end{array}$ \\
\hline 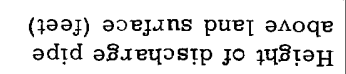 & & $\stackrel{\circ}{\dot{m}}$ & & $\stackrel{\sim}{\infty}$ & $\vec{i}$ & $\stackrel{\infty}{\infty}$. \\
\hline 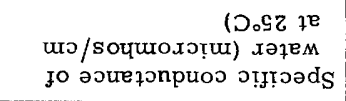 & $\vdots$ & $\begin{array}{ll}0 & 8 \\
0 & 8 \\
0 & 0 \\
0 & 0\end{array}$ & $\begin{array}{l}0 \\
0 \\
\infty \\
\infty \\
1\end{array}$ & 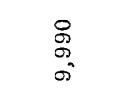 & $\begin{array}{l}i \\
i \\
\infty \\
\infty\end{array}$ & $\begin{array}{l}\stackrel{8}{0} \\
\infty\end{array}$ \\
\hline 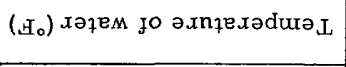 & $\vdots$ & $\stackrel{\sim}{\stackrel{D}{G}} \underset{\exists}{\stackrel{m}{二}}$ & & $\cong$ & $\stackrel{\circ}{\circ}$ & $\overrightarrow{0}$ \\
\hline хәғем јо әs $\Lambda$ & z & $\because \frac{02}{0}$ & 202 & n & $0^{2}$ & $a^{n}$ \\
\hline xəMod јо əd $K_{\mathrm{L}}$ & z & $\begin{array}{ll}\vdots & \vdots \\
\vdots & \vdots \\
\vdots & \vdots \\
\end{array}$ & 田国 & $\begin{array}{c}\vdots \\
\vdots \\
\vdots \\
\vdots\end{array}$ & $\vdots$ & 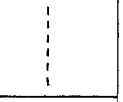 \\
\hline 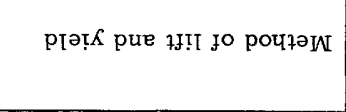 & $z$ & 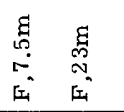 & $\ll \varangle$ & 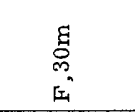 & 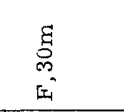 & 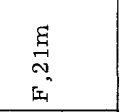 \\
\hline 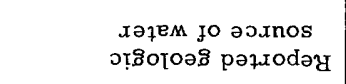 & 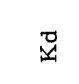 & 赵 & $\begin{array}{ll}\vdots & \vdots \\
\vdots & \vdots \\
\vdots & 1\end{array}$ & 8 & $\vdots$ & 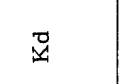 \\
\hline 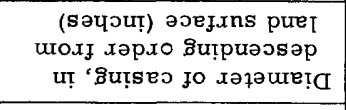 & $\stackrel{-1 / 4}{-1}$ & $\stackrel{-1+\infty}{\vec{m}} \stackrel{\text { के }}{m}$ & in & $\stackrel{N}{i s}$ & $\infty$ & 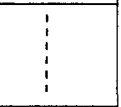 \\
\hline (ұәәј) чұđәр рәұходәч & 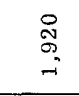 & $\begin{array}{l}\circ \\
\stackrel{\circ}{\circ} \\
i \\
i\end{array}$ & $\begin{array}{l:}1 \\
\vdots \\
\vdots \\
\vdots \\
1 \\
\end{array}$ & $\stackrel{\circ}{9}$ & $\begin{array}{l}8 \\
8 \\
\text { ก } \\
\end{array}$ & 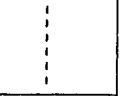 \\
\hline 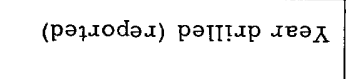 & 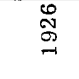 & 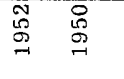 & $\begin{array}{l:c}\vdots \\
\vdots \\
\vdots \\
\vdots\end{array}$ & 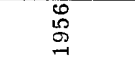 & $\begin{array}{l}0 \\
\stackrel{0}{0} \\
g\end{array}$ & 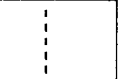 \\
\hline $\begin{array}{l}4 \\
0 \\
0 \\
5 \\
5 \\
0 \\
5 \\
0 \\
5 \\
0\end{array}$ & 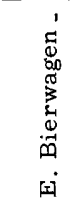 & 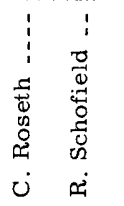 & 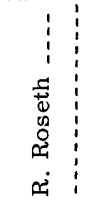 & 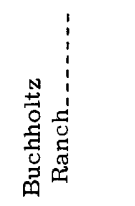 & 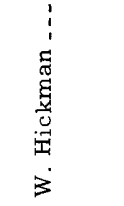 & $\begin{array}{l}1 \\
\vdots \\
\vdots \\
\vdots \\
\vdots \\
\vdots \\
\vdots \\
\vdots\end{array}$ \\
\hline $\overrightarrow{\bar{\phi}}$ & 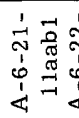 & 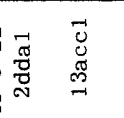 & 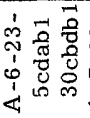 & 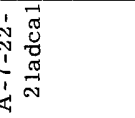 & 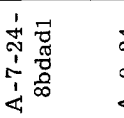 & 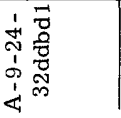 \\
\hline
\end{tabular}




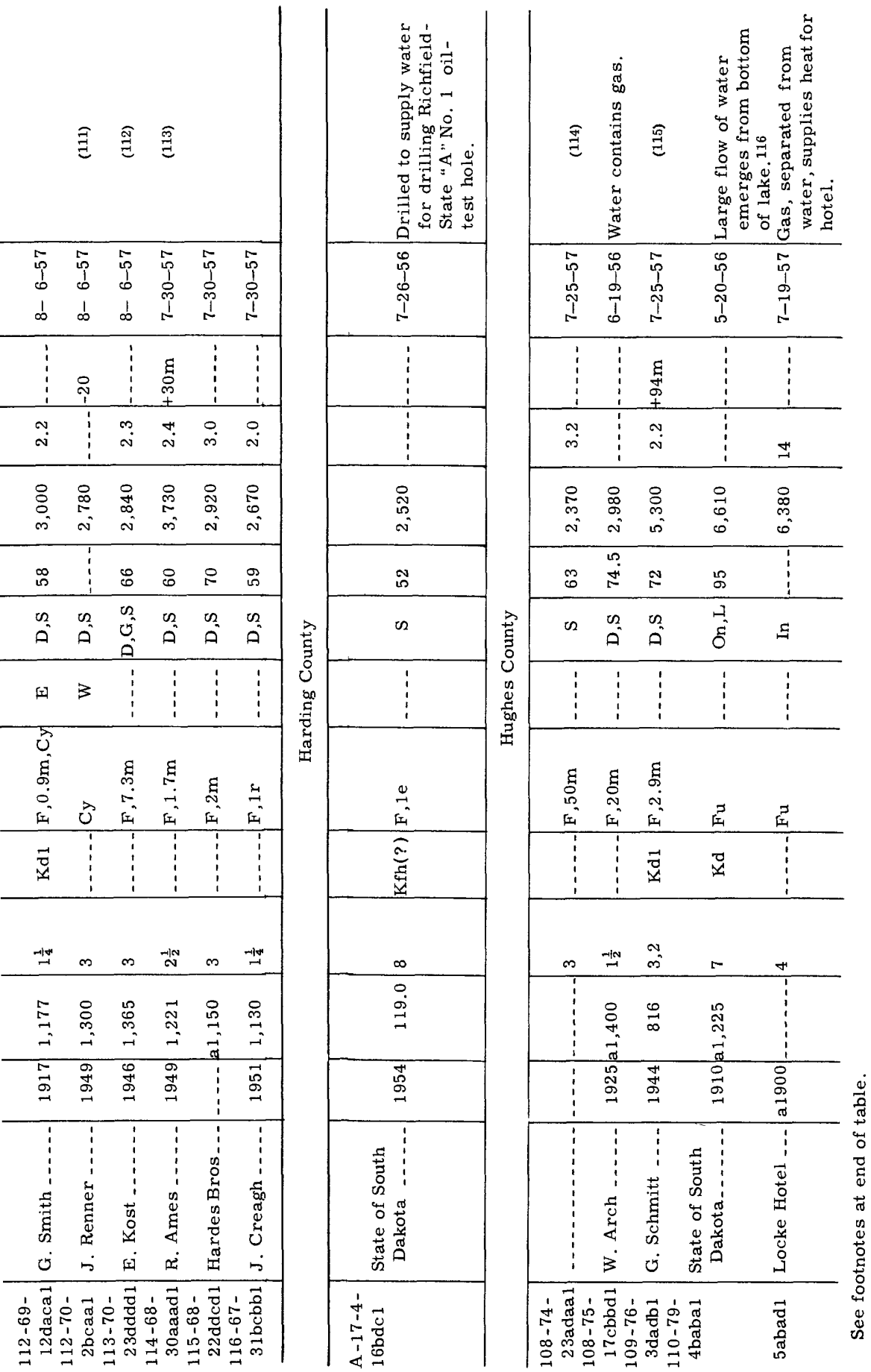




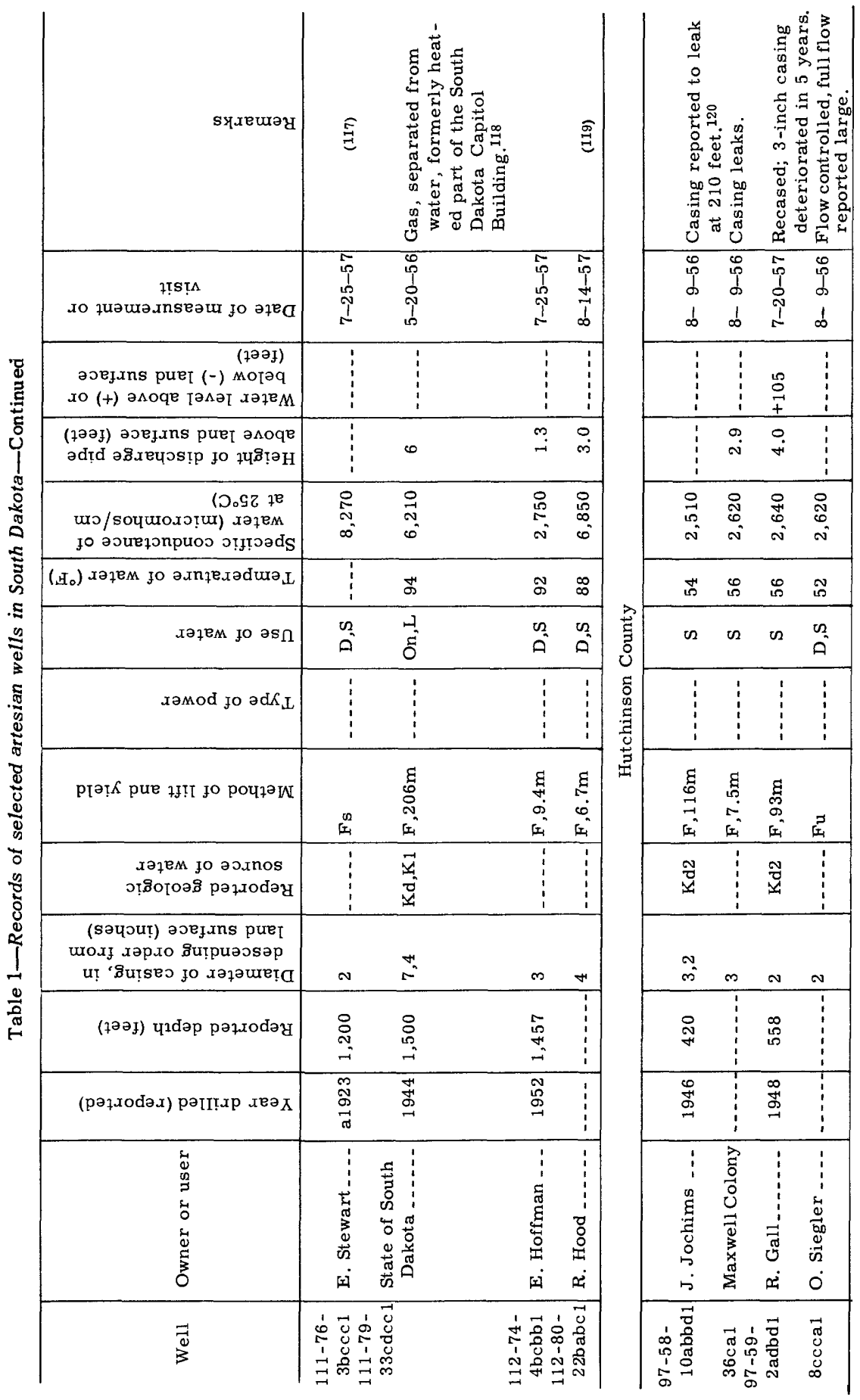




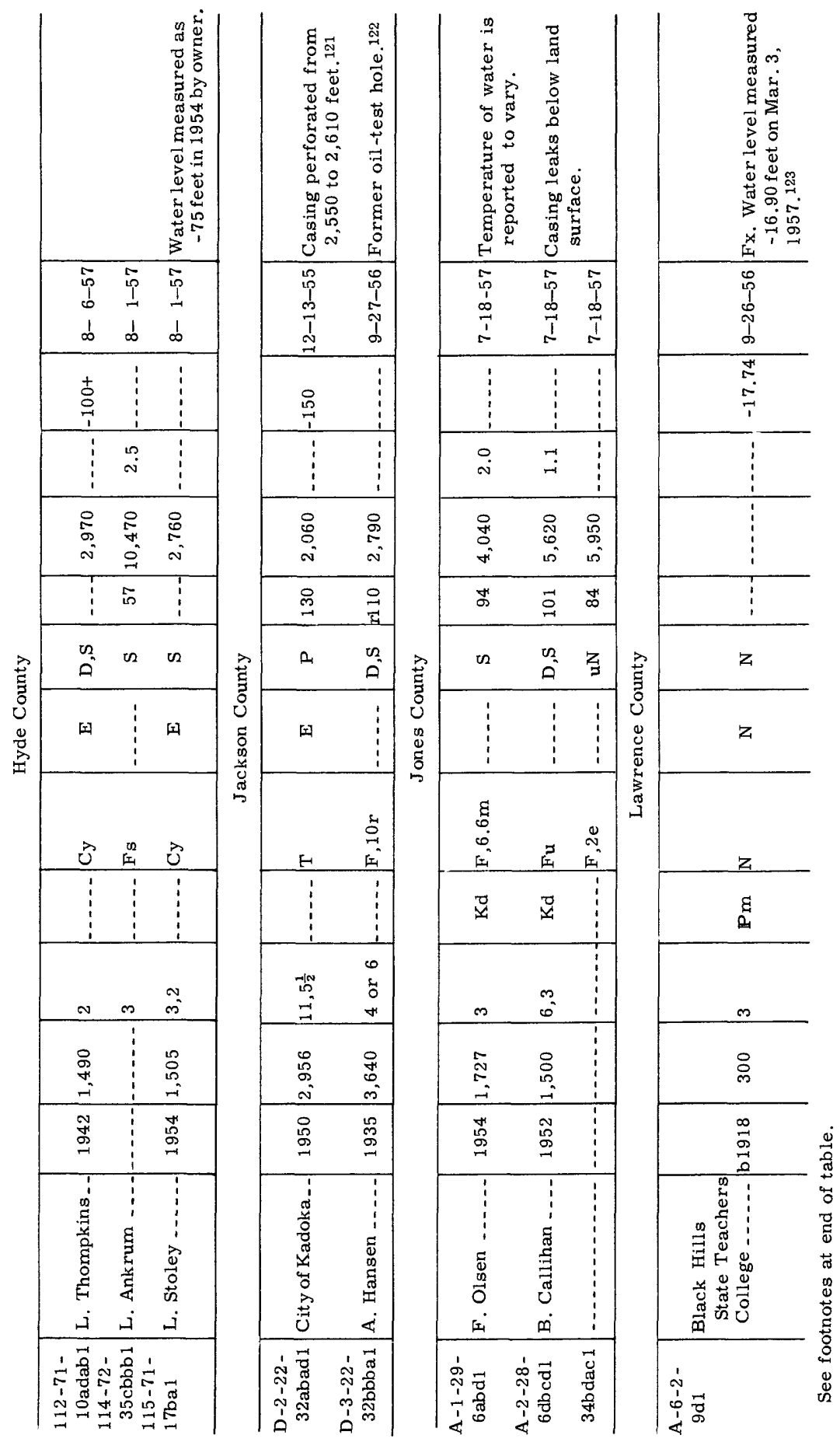




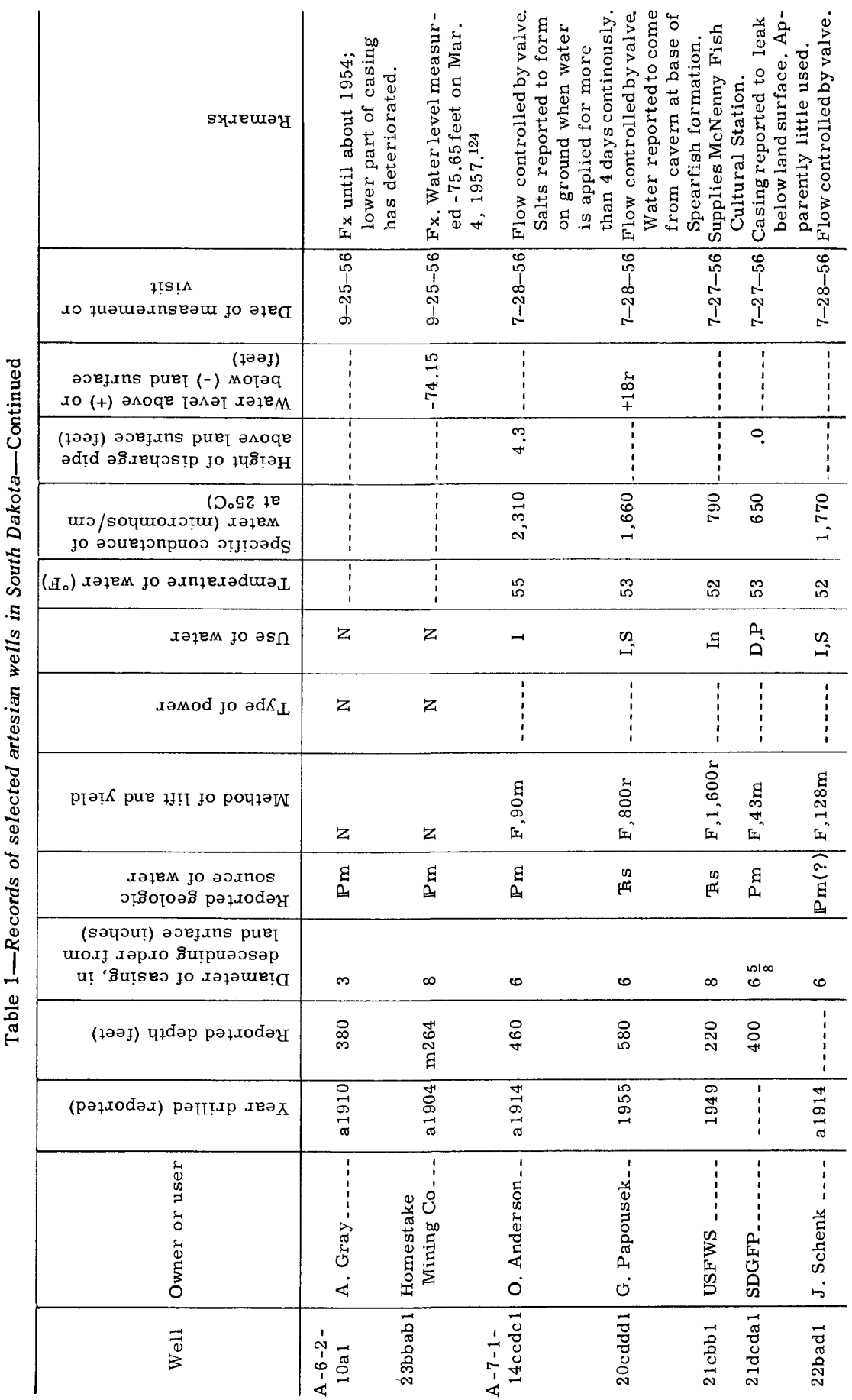



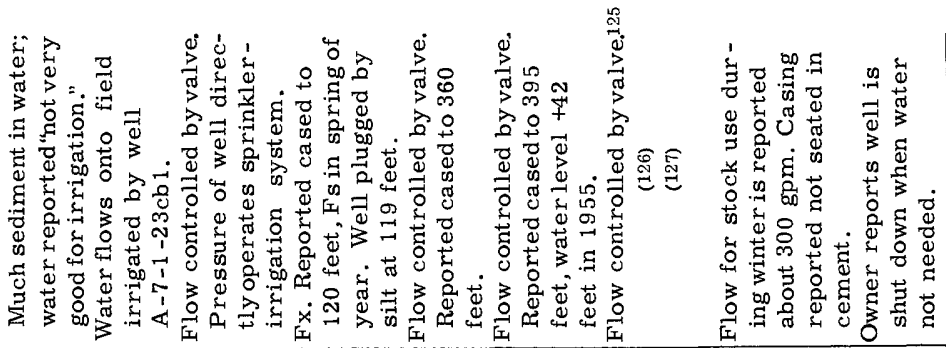

$\begin{array}{lllll}0 & 0 & 0 & 0 & 0 \\ 0 & 0 & 0 & 0 & 0 \\ b & 0 & 0 & 0 & 0 \\ 1 & 1 & 1 & 1 & 1\end{array}$

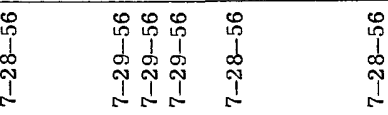

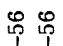

เป II

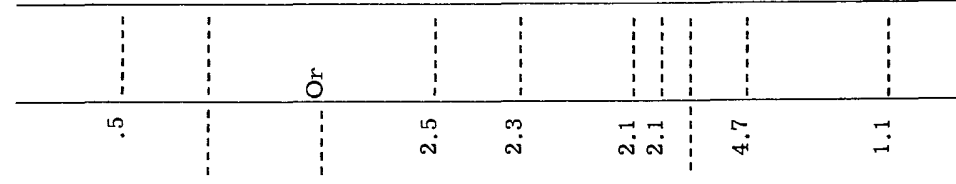

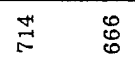

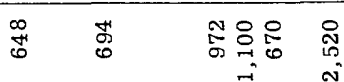

กิน กิ่

กิ กิร

药落:

ติ

$\rightarrow-1$

$\rightarrow$

$-$

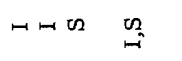

2

$\begin{array}{l:l:c} & z\end{array}$

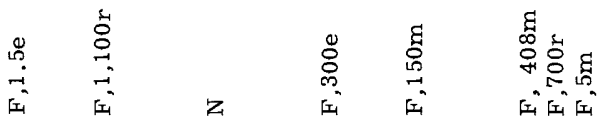

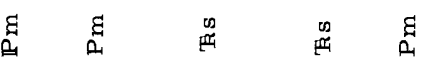

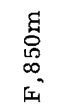

草

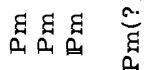

๕્વ

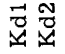

\begin{tabular}{|c|c|c|c|c|c|c|c|c|}
\hline 0 & \begin{tabular}{l}
$\infty$ \\
\multirow{\sim}{*}{}
\end{tabular} & 5 & $\infty$ & - & $\begin{array}{lll}\infty & 0 & 1 \\
0 & 0 & 0 \\
0 & 0 & 0\end{array}$ & เด & $\forall$ & $\forall N$ \\
\hline 禹 & $\stackrel{8}{0}$ & స్ల & $\vec{\exists}$ & 品 & 号 品 品 & $\stackrel{\sim}{*}$ & $\stackrel{8}{:}$ & $\begin{array}{l}\text { : : } \\
\text { : } \\
\text { : }\end{array}$ \\
\hline 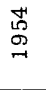 & $\begin{array}{l}\text { 占 } \\
\text { م } \\
-1\end{array}$ & $\begin{array}{l}0 \\
\sigma \\
\sigma \\
\sigma\end{array}$ & $\begin{array}{l}\ddot{\varpi} \\
\stackrel{\sigma}{\sigma}\end{array}$ & $\begin{array}{l}\text { 号 } \\
\text { 品 }\end{array}$ & 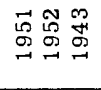 & $\begin{array}{l}\text { 苟 } \\
\text { の }\end{array}$ & $\begin{array}{l}\text { 号 } \\
\stackrel{8}{\Omega}\end{array}$ & 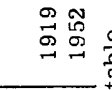 \\
\hline 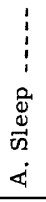 & $\begin{array}{l}\vdots \\
\vdots \\
\vdots \\
\vdots \\
\vdots \\
\vdots \\
\vdots\end{array}$ & $\begin{array}{c}1 \\
\vdots \\
0 \\
0 \\
0 \\
0 \\
0 \\
0 \\
0 \\
0 \\
0 \\
0\end{array}$ & $\begin{array}{r}1 \\
\vdots \\
\vdots \\
\vdots \\
\vdots \\
\vdots \\
\vdots \\
\end{array}$ & $\begin{array}{r}1 \\
\vdots \\
\vdots \\
\vdots \\
\vdots \\
\vdots \\
\vdots \\
\end{array}$ & 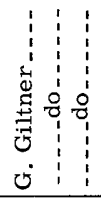 & 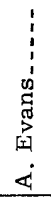 & $\begin{array}{l}1 \\
0 \\
8 \\
8 \\
3 \\
0 \\
0 \\
4 \\
1\end{array}$ & 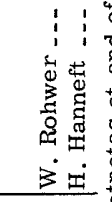 \\
\hline $\begin{array}{l}\overrightarrow{0} \\
\text { ले } \\
\text { N }\end{array}$ & $\begin{array}{l}\overrightarrow{0} \\
\text { Oे } \\
\text { N }\end{array}$ & 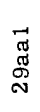 & $\begin{array}{l}\text { N } \\
\text { ळ్ } \\
\text { N }\end{array}$ & $\begin{array}{l}\text { m } \\
\text { J } \\
\text { న్ }\end{array}$ & 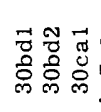 & $\begin{array}{l}\vec{\pi} \\
0 \\
0 \\
0\end{array}$ & $\begin{array}{l}\vec{z} \\
\tilde{J} \\
\tilde{N} \\
\text { N }\end{array}$ & 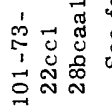 \\
\hline
\end{tabular}




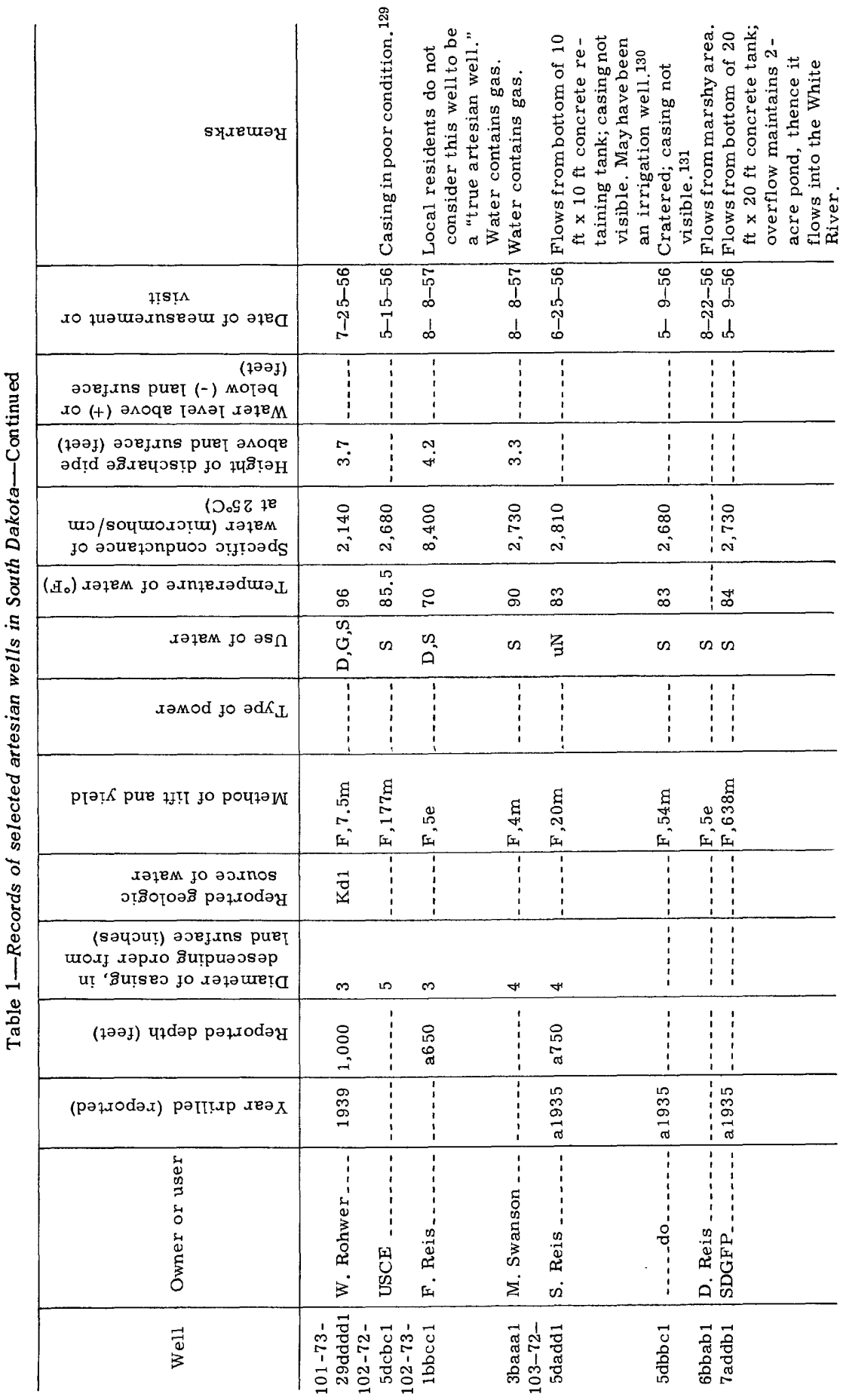




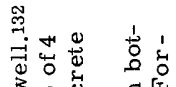

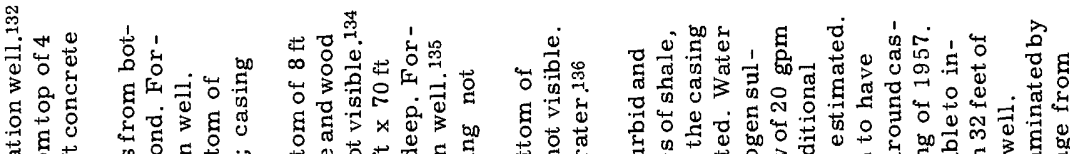

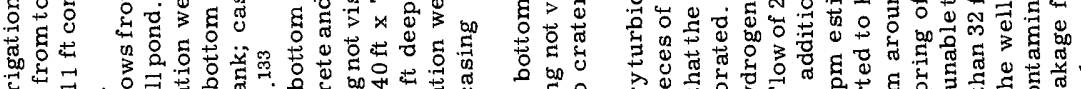

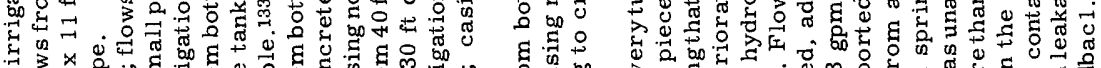

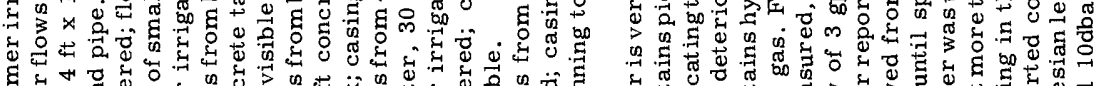

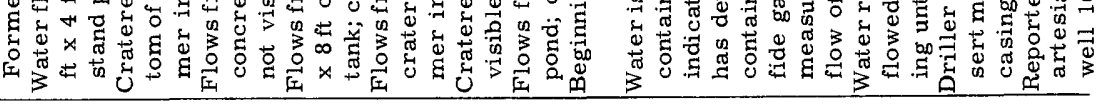

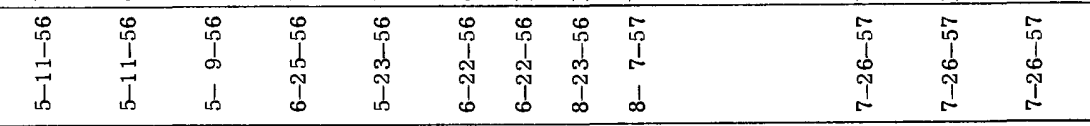

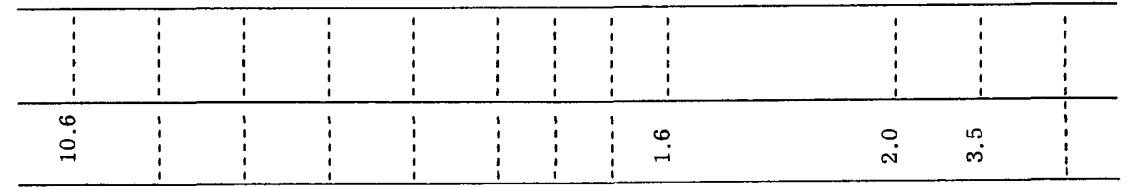

\begin{tabular}{|c|c|c|c|c|c|c|c|c|c|c|c|}
\hline $\begin{array}{l}\stackrel{D}{N} \\
\text { i. }\end{array}$ & 亲 & $\begin{array}{l}\text { 焉 } \\
\text { in }\end{array}$ & $\underset{\sim}{\stackrel{D}{\circ}}$ & $\begin{array}{l}\text { I } \\
\text { t. } \\
\text { in }\end{array}$ & $\begin{array}{l}\circ \\
\text { م. } \\
\text { o }\end{array}$ & 吕 & & $\begin{array}{l}\stackrel{\circ}{-} \\
m^{\circ}\end{array}$ & 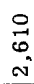 & $\begin{array}{l}0 \\
0 \\
0 \\
\text { in }\end{array}$ & $\begin{array}{l}\text { o } \\
\text { o } \\
\text { i }\end{array}$ \\
\hline 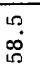 & $\infty_{\infty}$ & ㅇ & $\begin{array}{l}10 \\
\infty \\
\infty\end{array}$ & $\mathscr{\infty}_{\infty}$ & No & ऽ) & চ్ & $\vec{\sigma}$ & $\begin{array}{l}\infty \\
0 \\
0 \\
0\end{array}$ & ৪ & \\
\hline
\end{tabular}

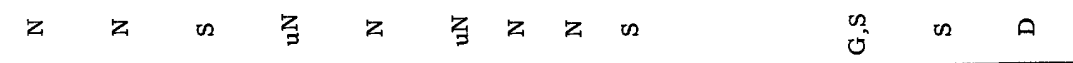

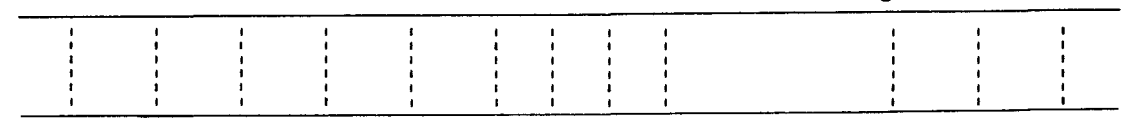

\begin{tabular}{|c|c|c|c|c|c|c|c|c|c|c|c|}
\hline ⿷匚 & 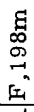 & 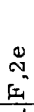 & $\begin{array}{l}\xi \\
\circ \\
\circ \\
\dot{E}\end{array}$ & $\begin{array}{l}\Xi \\
\text { J } \\
\text { N } \\
\text { E }\end{array}$ & 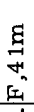 & 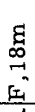 & 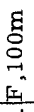 & 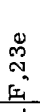 & 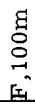 & 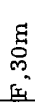 & \\
\hline 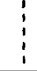 & $\vdots$ & 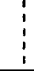 & & & 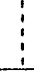 & $i$ & & 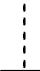 & 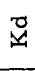 & ? & శే \\
\hline 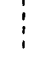 & 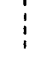 & 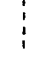 & 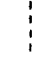 & 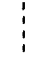 & 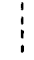 & 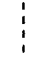 & $\omega$ & $m$ & $m$ & $\omega$ & 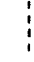 \\
\hline
\end{tabular}

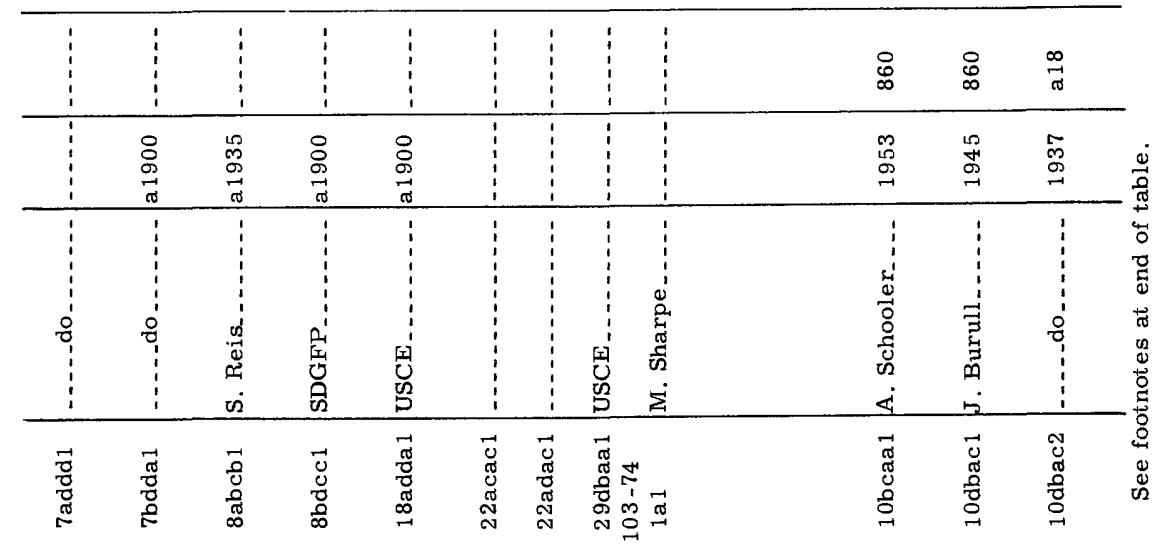




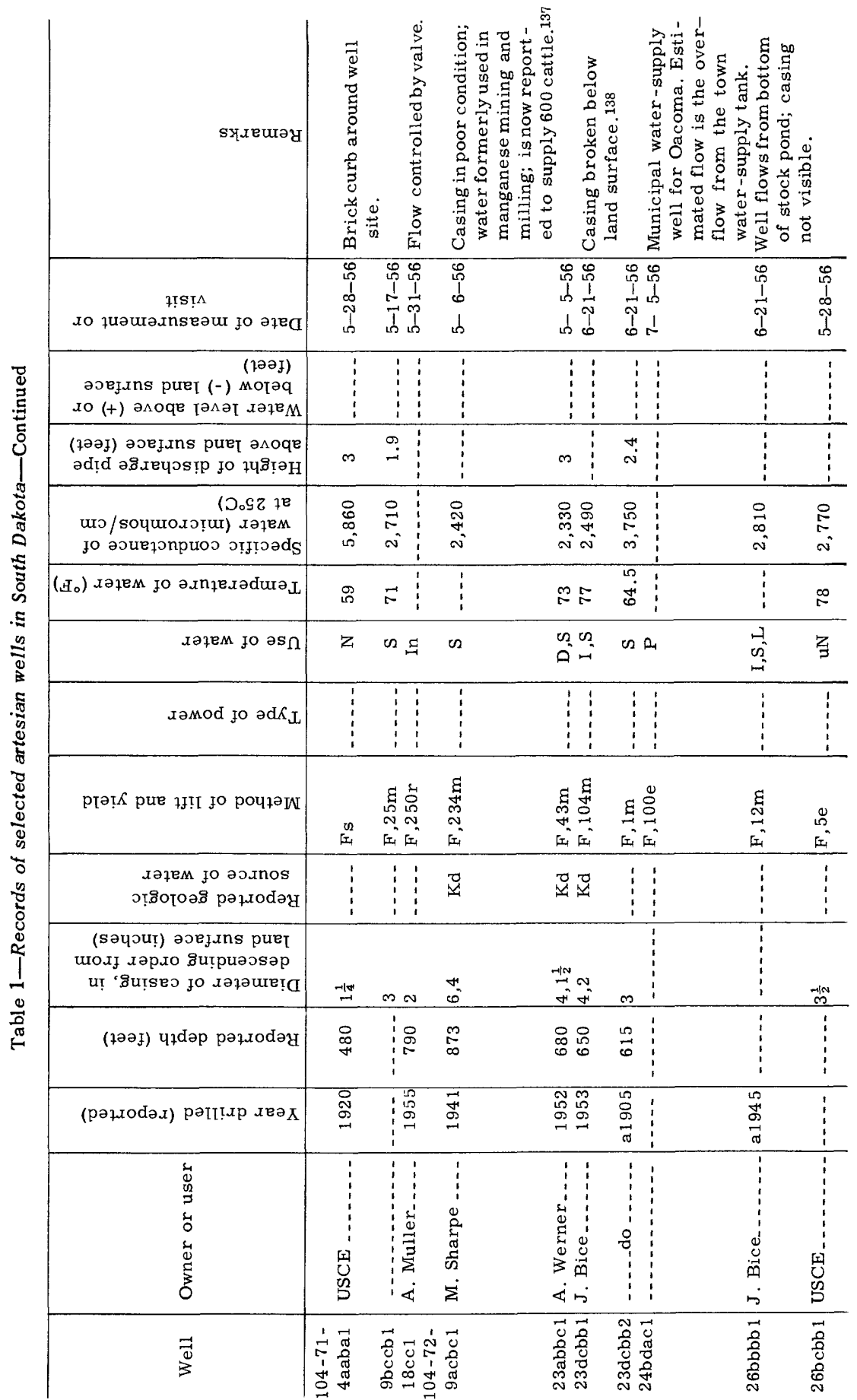




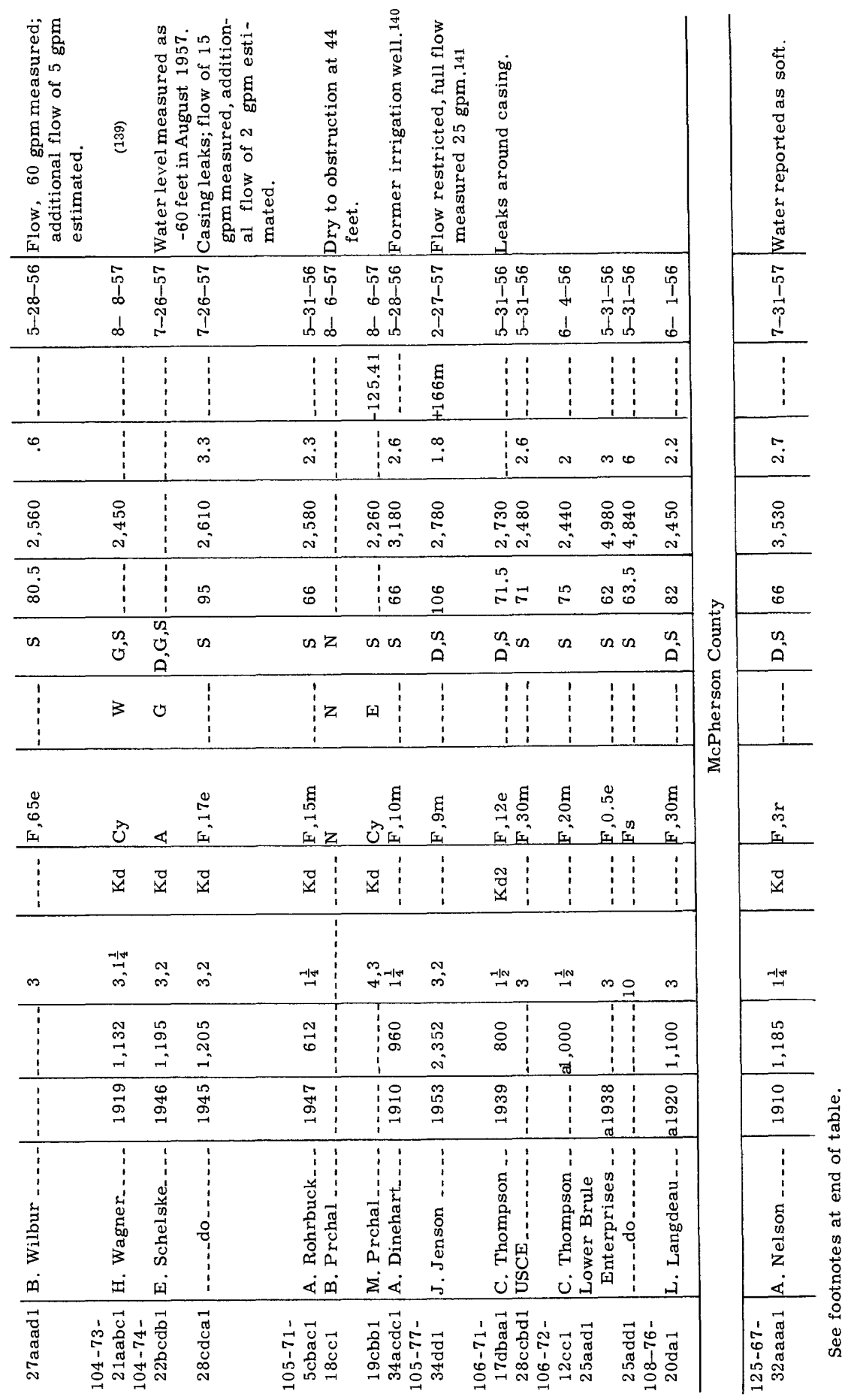




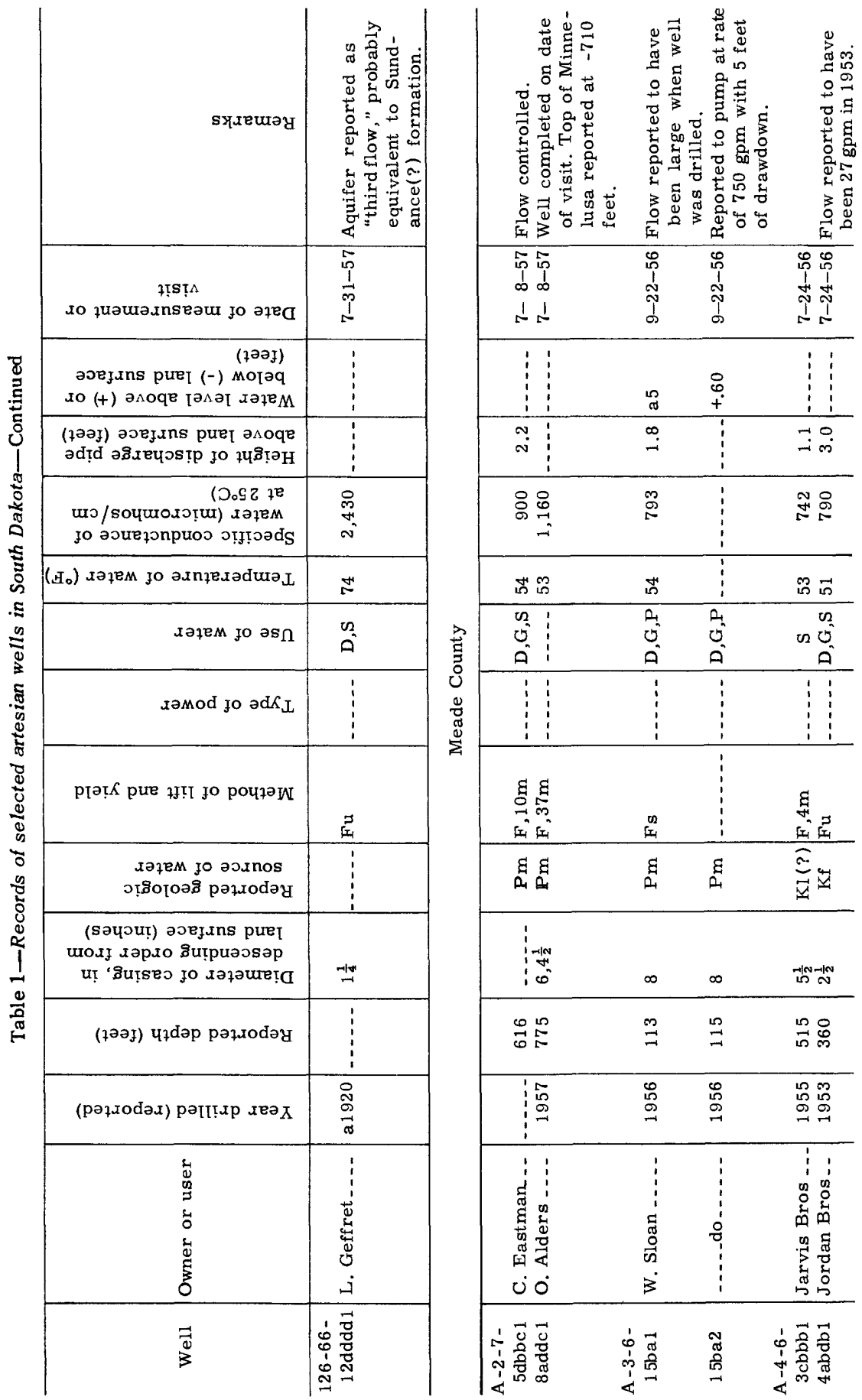




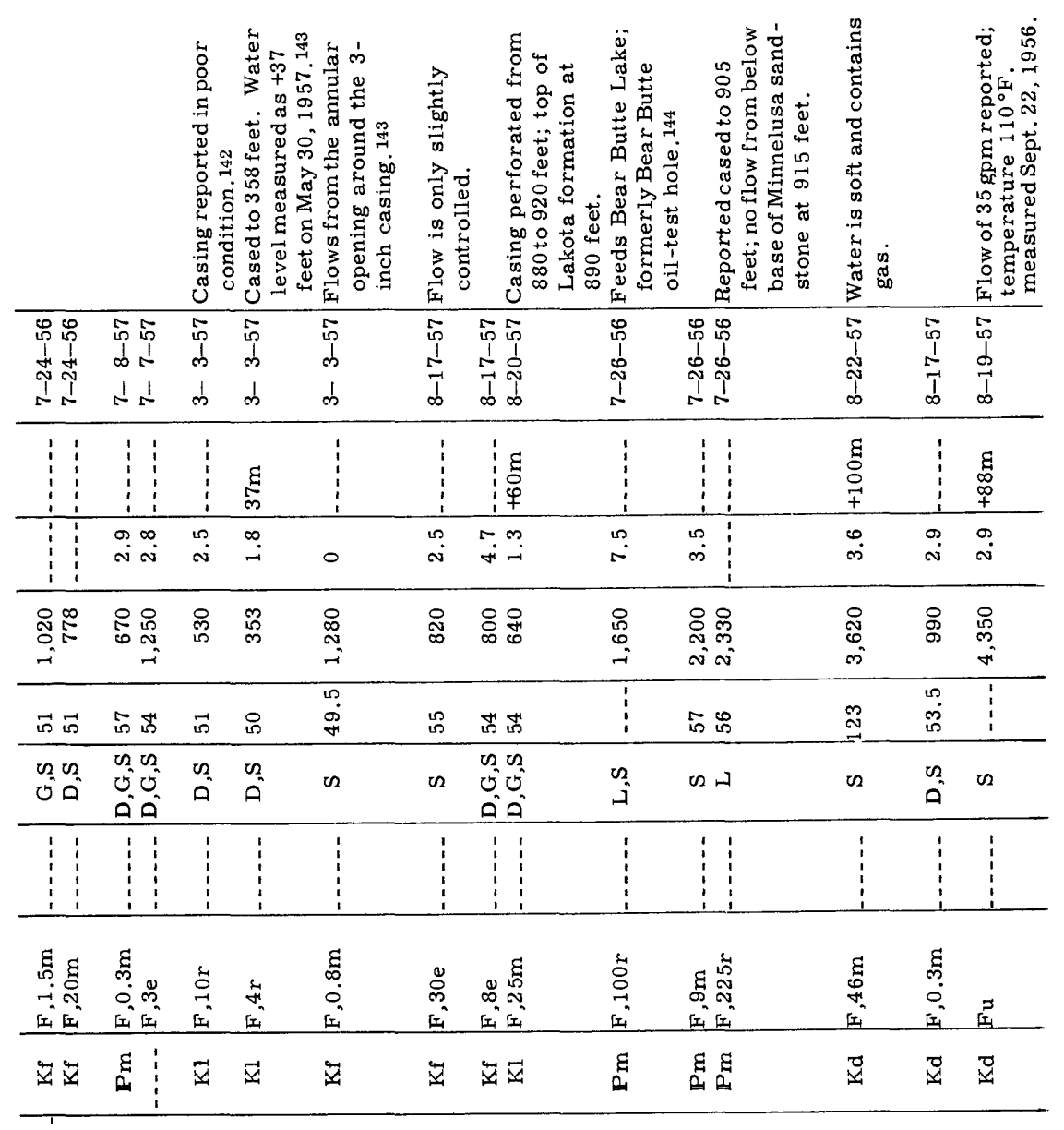

\begin{tabular}{|c|c|c|c|c|c|c|c|c|c|c|c|}
\hline $\begin{array}{l}-\mathbb{N} \\
N\end{array}$ & $\sim \stackrel{-l N N}{N}$ & 0 & $\infty$ & $\stackrel{+}{m}$ & $m$ & $\sim \curvearrowright$ & $\infty$ & $\begin{array}{r}\infty \\
\sim \infty^{\circ}\end{array}$ & $\begin{array}{l}N \\
\infty\end{array}$ & N & $m$ \\
\hline 안 & \begin{tabular}{l:}
\multirow{H}{*}{} \\
\hdashline
\end{tabular} & 总 & ஓ & 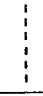 & $\stackrel{\infty}{\stackrel{N}{N}}$ & స్ & 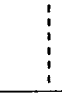 & 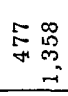 & $\begin{array}{l}\stackrel{N}{*} \\
\stackrel{\sim}{*}\end{array}$ & 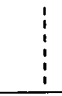 & 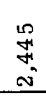 \\
\hline 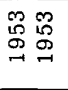 & 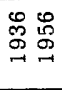 & $\vec{\infty}$ & 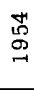 & $\underset{\mathrm{S}}{\stackrel{\mathrm{g}}{\mathrm{g}}}$ & $\stackrel{\mathscr{D}}{\mathscr{\sigma}}$ & 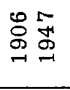 & $\underset{\sigma}{\sigma}$ & 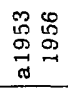 & 占 & $\vdots$ & $\begin{array}{l}\stackrel{\circ}{\circ} \\
\stackrel{5}{-}\end{array}$ \\
\hline $\begin{array}{r:} \\
1 \\
1 \\
1\end{array}$ & 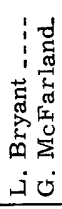 & 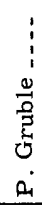 & $\begin{array}{l}\vdots \\
\vdots \\
\vdots \\
1\end{array}$ & $\begin{array}{r}\vdots \\
\vdots \\
\vdots \\
\vdots \\
\hdashline\end{array}$ & 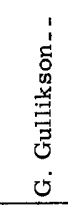 & 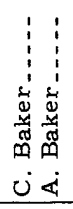 & 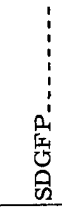 & 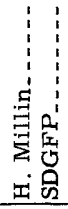 & 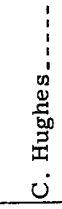 & 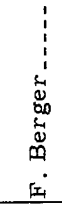 & 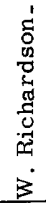 \\
\hline 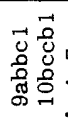 & \begin{tabular}{ll}
$\overrightarrow{0}$ & \multicolumn{2}{c}{} \\
0 & 0 \\
0 & 0 \\
0 & 0 \\
0 & 0
\end{tabular} & 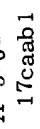 & 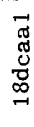 & 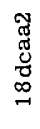 & 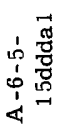 & 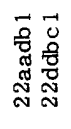 & 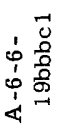 & 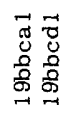 & $\begin{array}{ll}1 & \overrightarrow{0} \\
\overrightarrow{0} & 0 \\
\dot{b} & 0 \\
1 & 0 \\
1 & 0\end{array}$ & 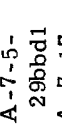 & d্] \\
\hline
\end{tabular}




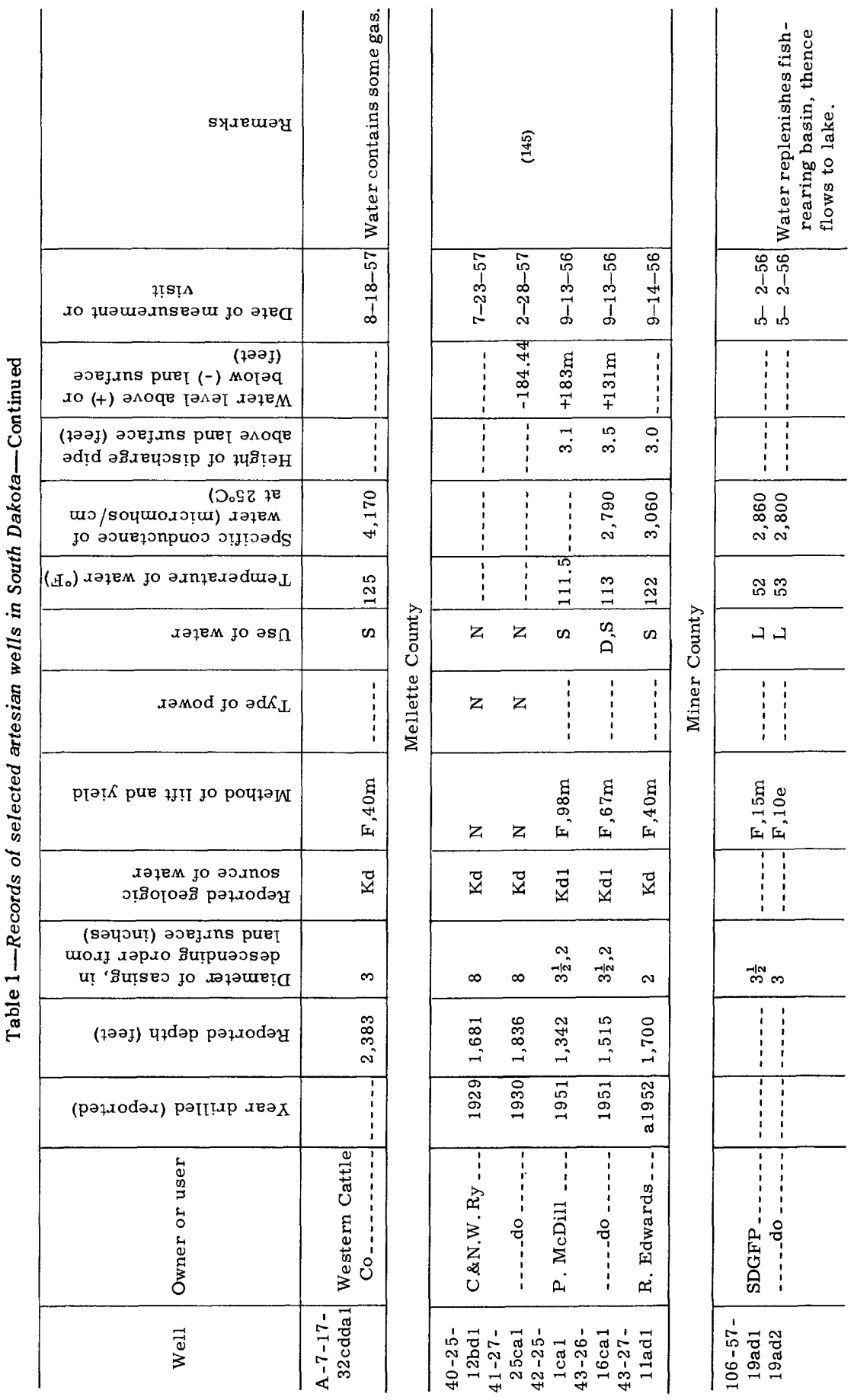




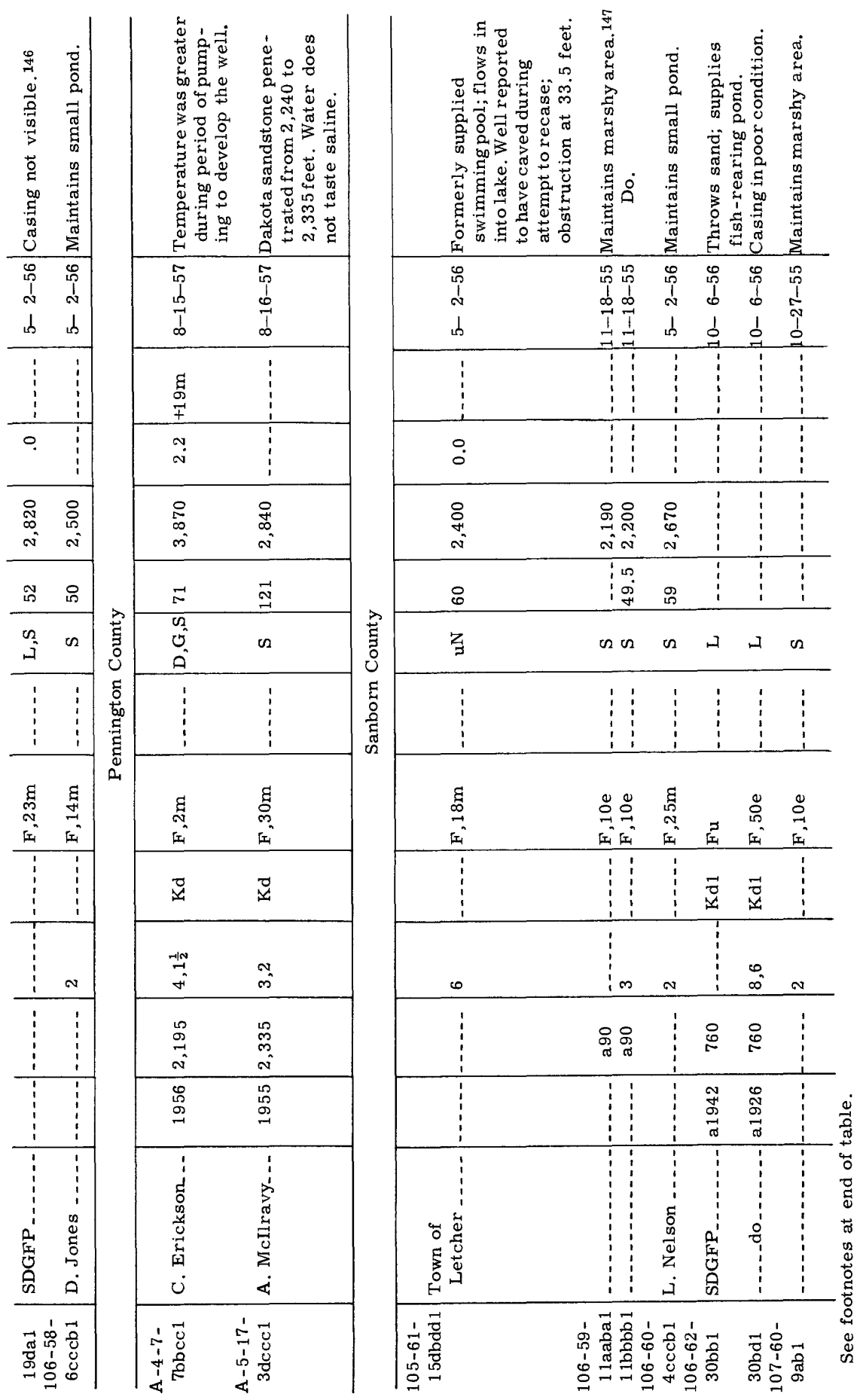




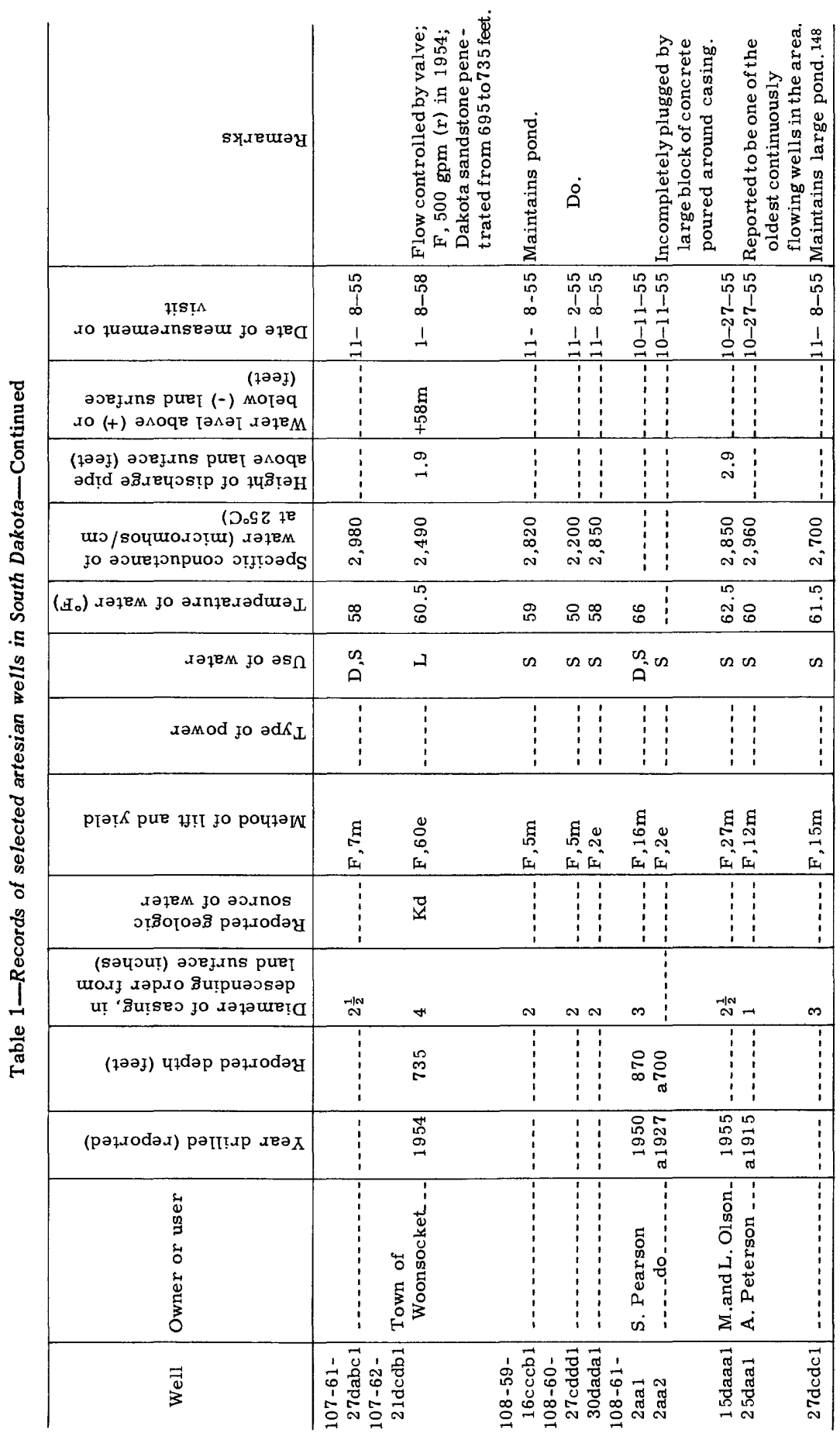




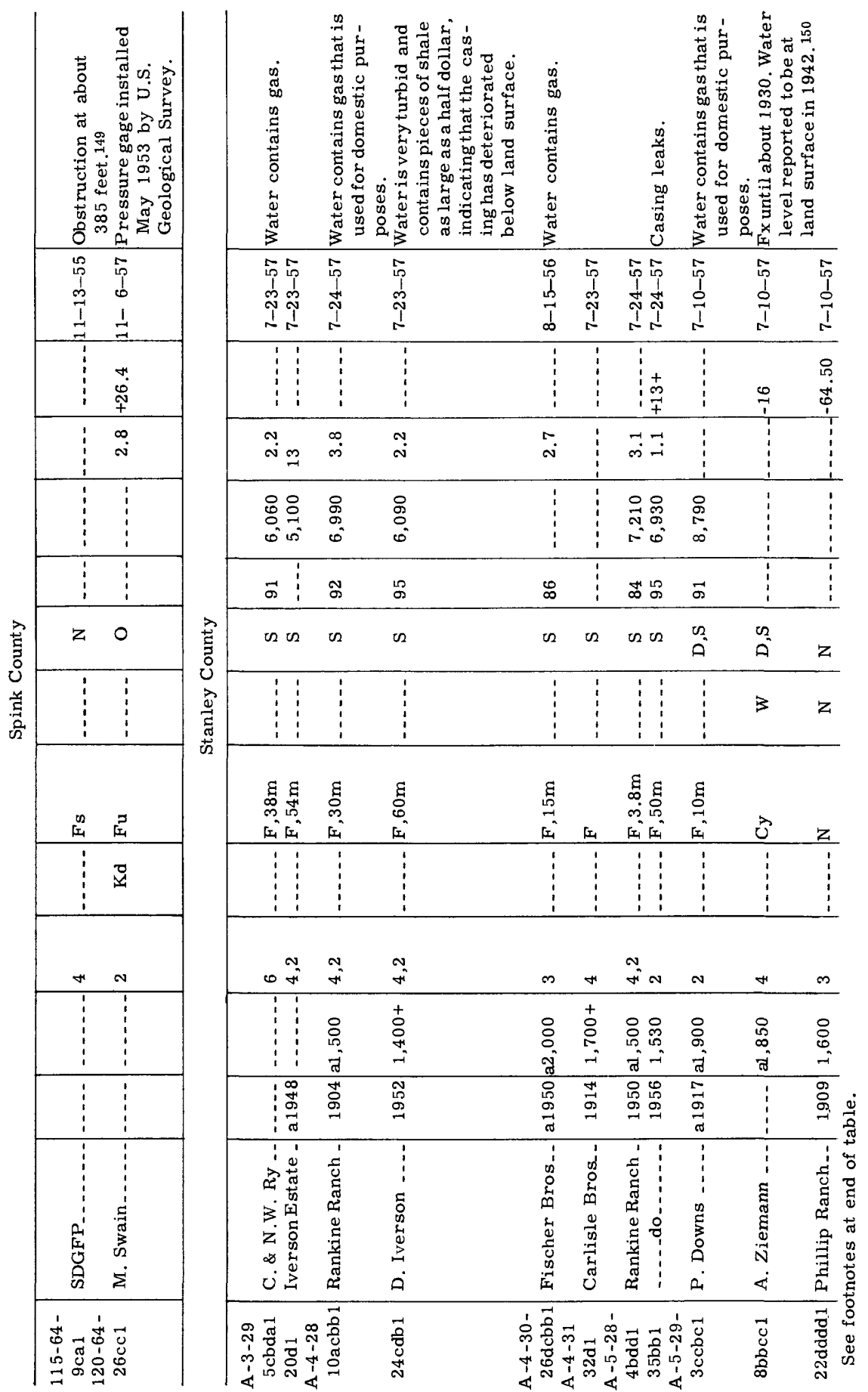




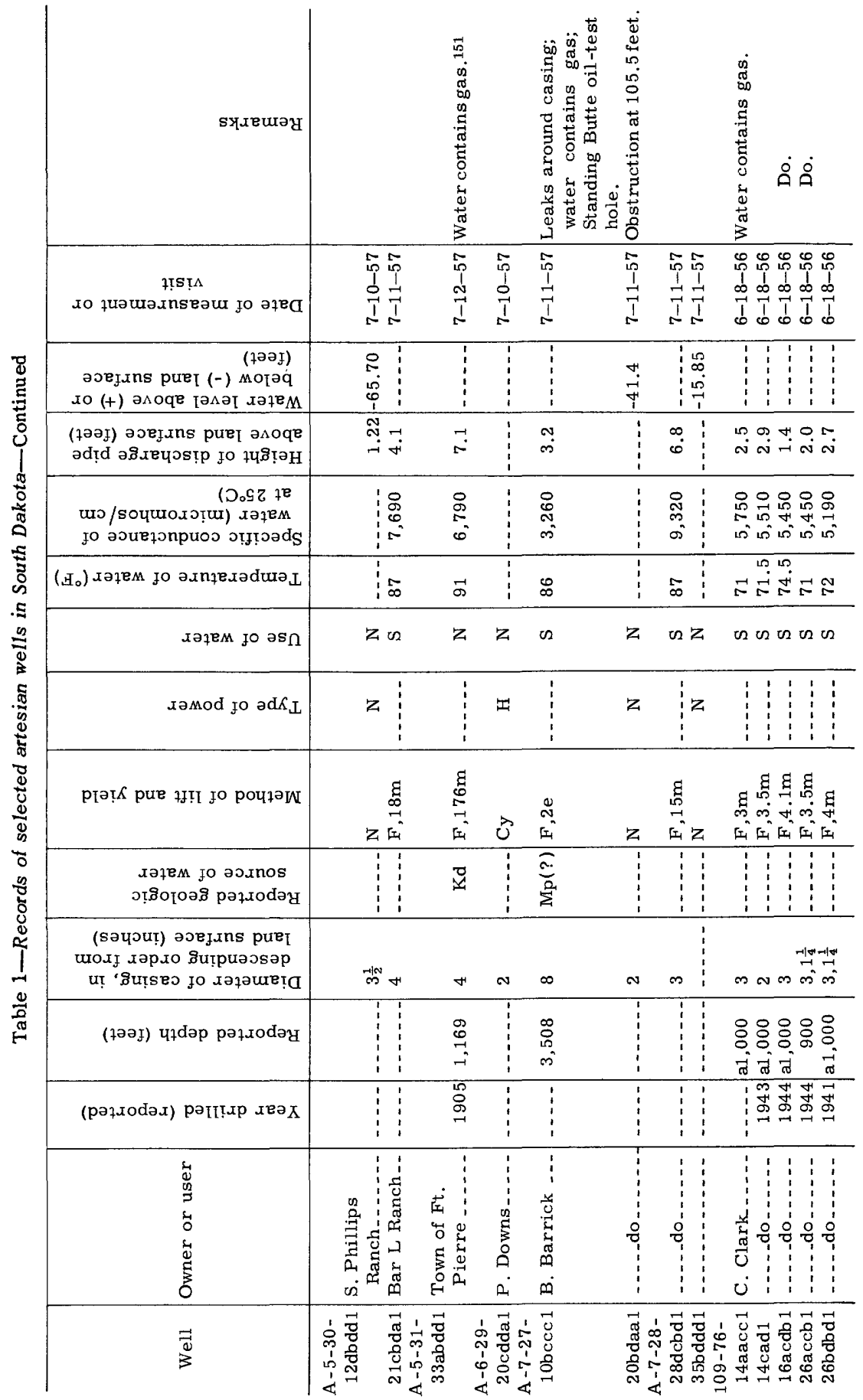




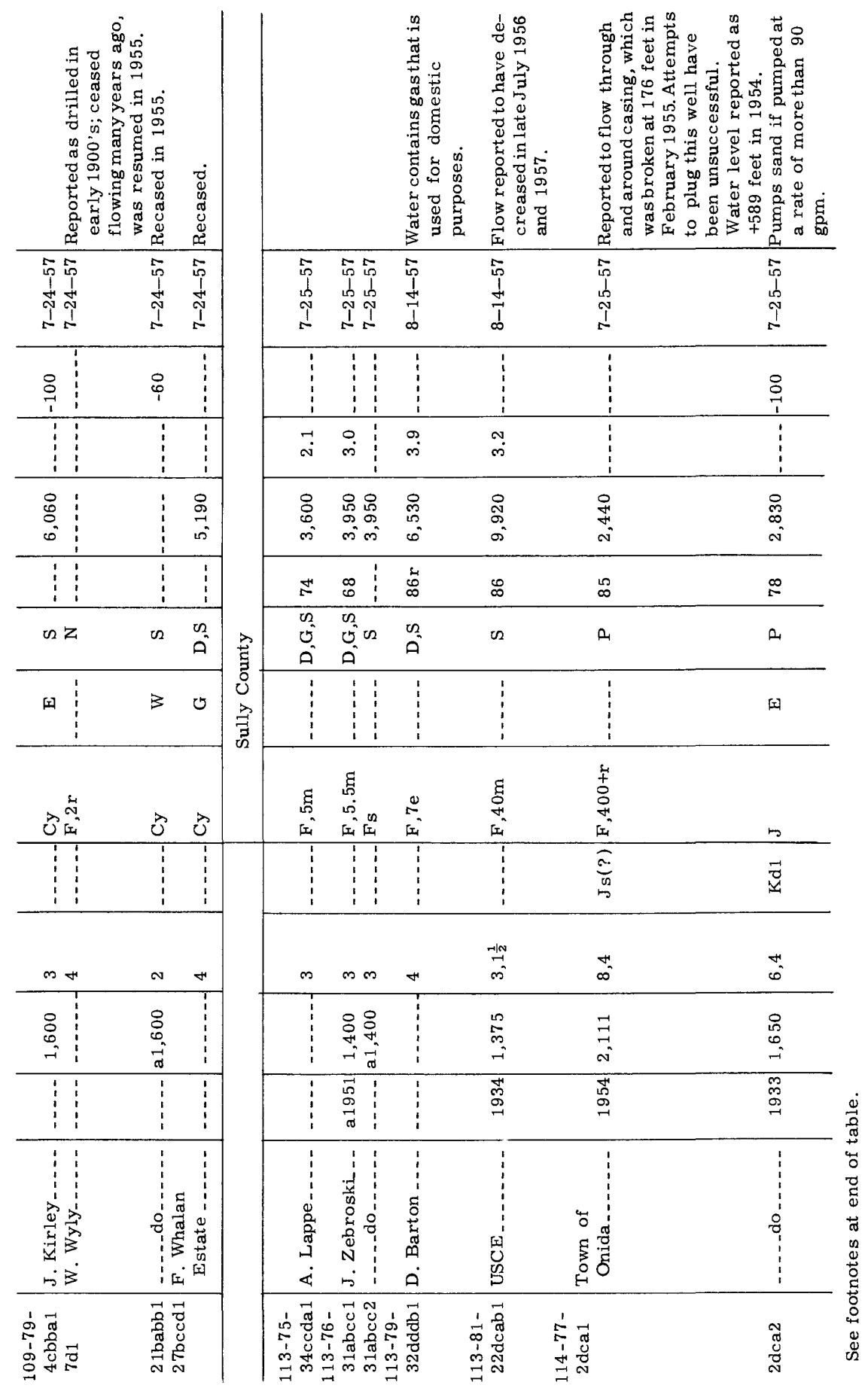




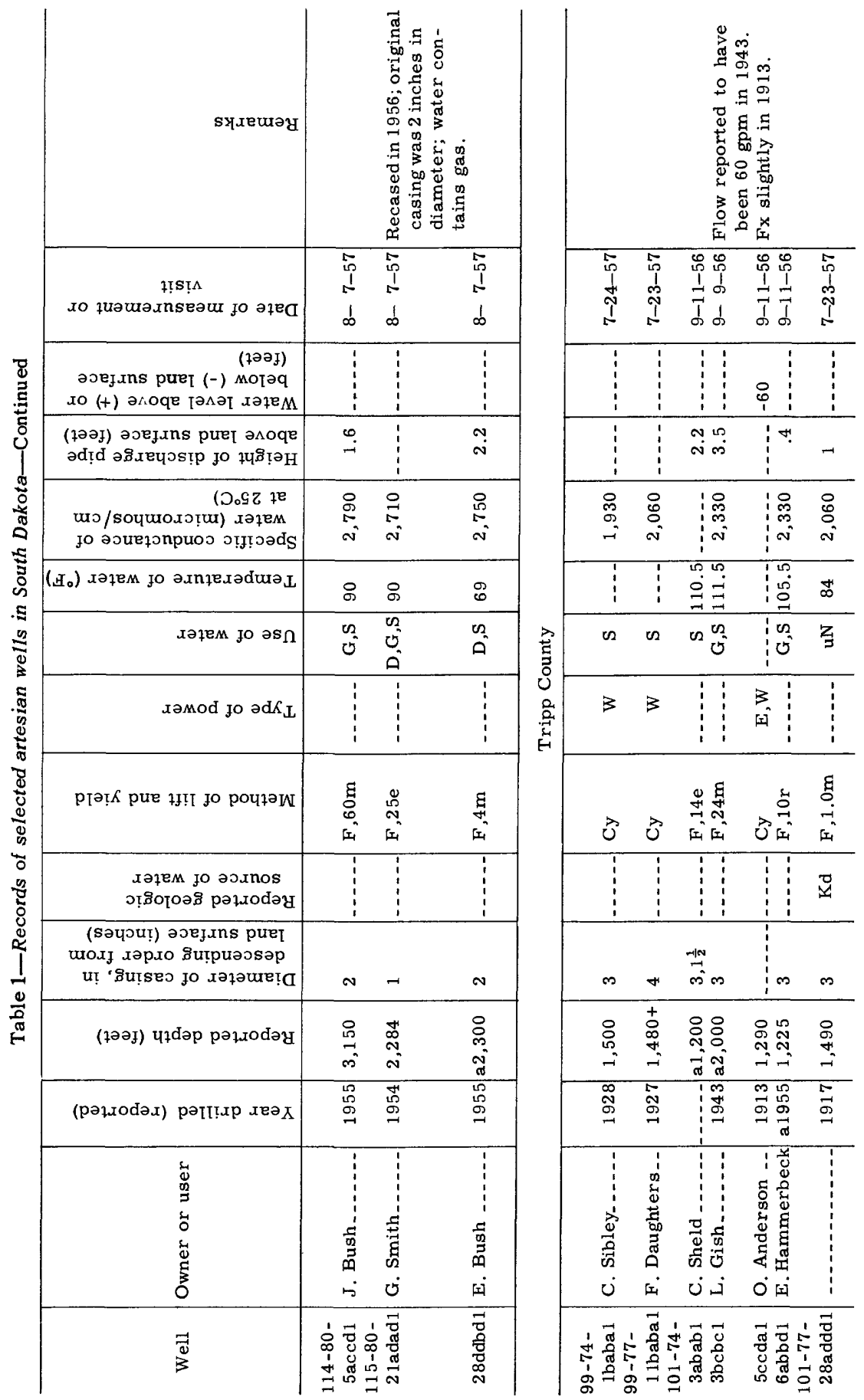




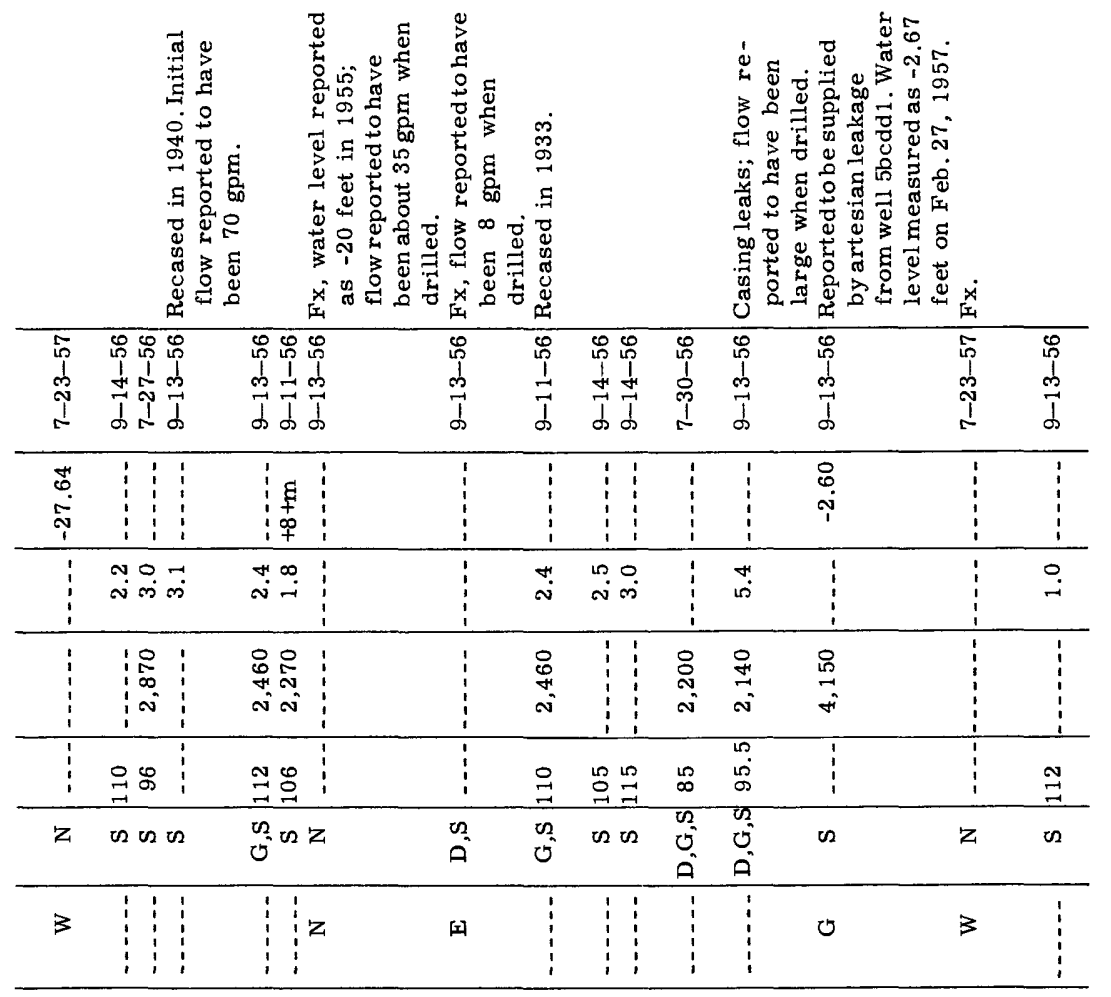

\begin{tabular}{|c|c|c|c|c|c|c|c|c|c|c|}
\hline$\vec{U}$ & 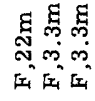 & 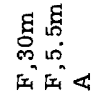 & $\varangle$ & 䓃 & 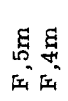 & 屈 & घू & $\vec{u}$ & $\vec{v}$ & 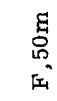 \\
\hline T. & & & $\vec{\nabla}$ & 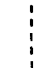 & & $\vec{\nabla}$ & & $\sigma$ & $\vec{x}$ & \\
\hline
\end{tabular}

\begin{tabular}{|c|c|c|c|c|c|c|c|c|c|c|}
\hline$H$ & $\begin{array}{l:ll}N & \\
\infty & N\end{array}$ & 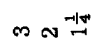 & के & $\stackrel{-1 \mid r}{-1}$ & ${ }^{\infty 14}{ }_{\text {in }}$ & $m$ & $H$ & $\stackrel{\Delta}{\sim}$ & $m$ & N \\
\hline $\begin{array}{l}8 \\
8 \\
-1 \\
-1\end{array}$ & 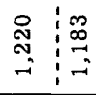 & 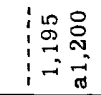 & $\begin{array}{l}\text { 今 } \\
\text { సે } \\
-\end{array}$ & $\begin{array}{l}\text { In } \\
\text { N } \\
-i \\
-1\end{array}$ & $\begin{array}{l:}8 \\
\stackrel{0}{-1} \\
\Rightarrow\end{array}$ & 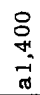 & : & में & $\begin{array}{l}\text { 음 } \\
\text { in } \\
\text { नi }\end{array}$ & 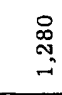 \\
\hline$\vec{\sigma}$ & $\begin{array}{lll} & 1 & 1 \\
& & 1 \\
& & 1 \\
& 1 & 1 \\
\end{array}$ & 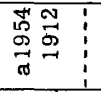 & $\begin{array}{l}\overrightarrow{5} \\
\text { के }\end{array}$ & \begin{tabular}{l}
$\infty$ \\
\multirow{J}{0}{} \\
$\pi$ \\
$\pi$
\end{tabular} & 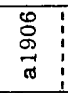 & $\begin{array}{l}0 \\
0 \\
-9 \\
-1\end{array}$ & $\begin{array}{l}\infty \\
\stackrel{\sigma}{\sigma}\end{array}$ & 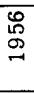 & 욱 & $\begin{array}{l}\text { 品 } \\
\stackrel{9}{\circ}\end{array}$ \\
\hline 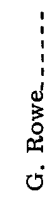 & 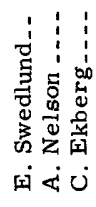 & 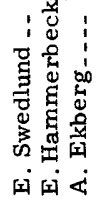 & $\begin{array}{l}1 \\
\vdots \\
\vdots \\
\vdots \\
\vdots \\
\vdots \\
\vdots\end{array}$ & 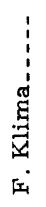 & 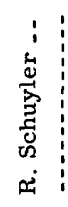 & 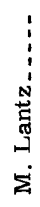 & 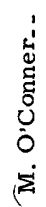 & $\begin{array}{l}\vdots \\
\vdots \\
\vdots \\
\vdots \\
\vdots \\
\vdots \\
\vdots\end{array}$ & ض. & 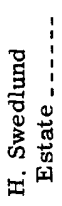 \\
\hline $\begin{array}{l}1 \overrightarrow{0} \\
0 \\
0 \\
1 \\
0 \\
0 \\
0 \\
0 \\
0\end{array}$ & 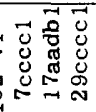 & 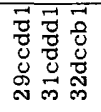 & 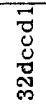 & $\begin{array}{l}\vec{\pi} \\
\text { o } \\
\text { O } \\
\text { Jै }\end{array}$ & 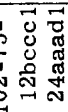 & & & 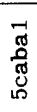 & $\begin{array}{l}\vec{z} \\
\bar{z} \\
\overline{0} \\
\bar{n}\end{array}$ & \\
\hline
\end{tabular}




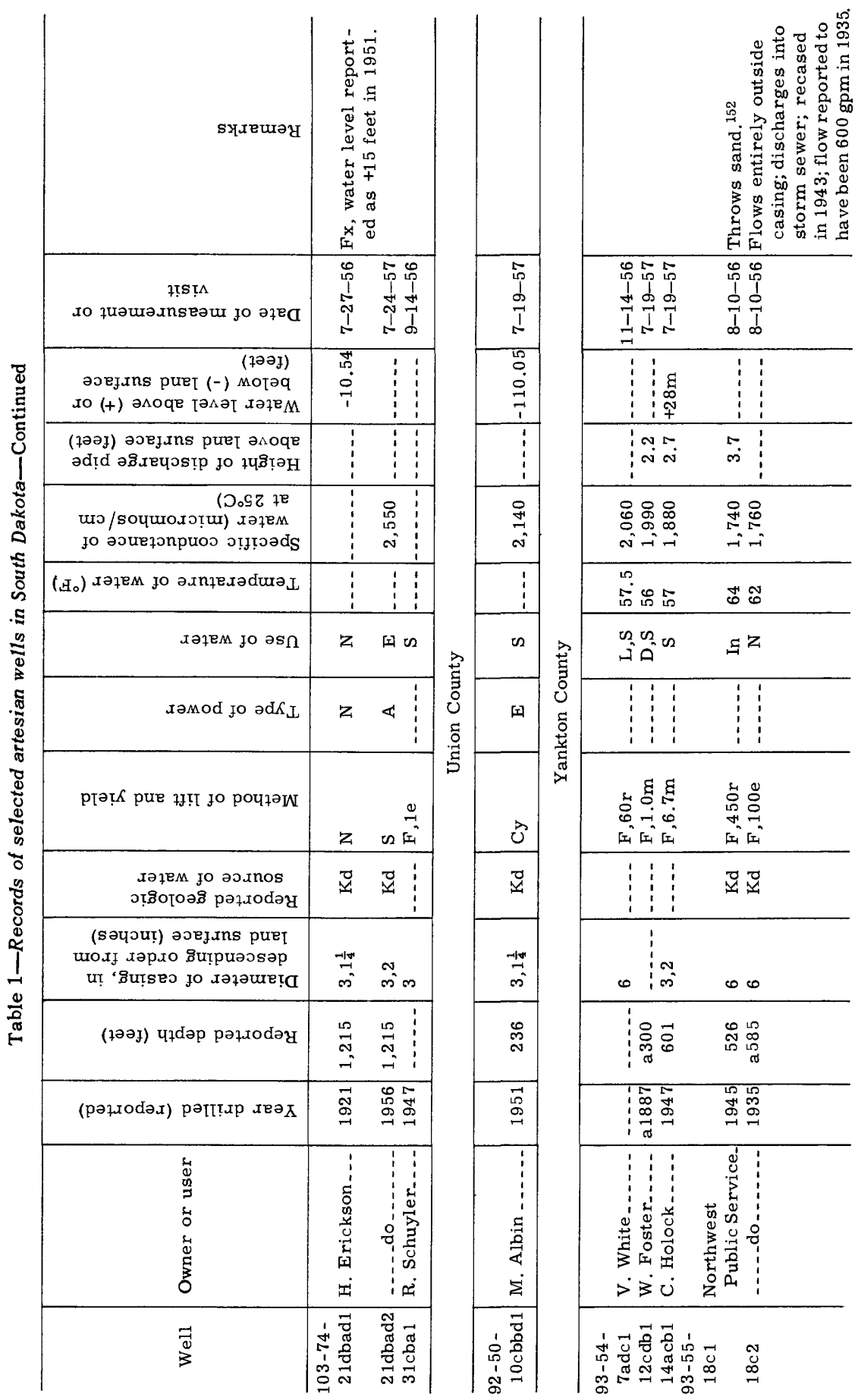




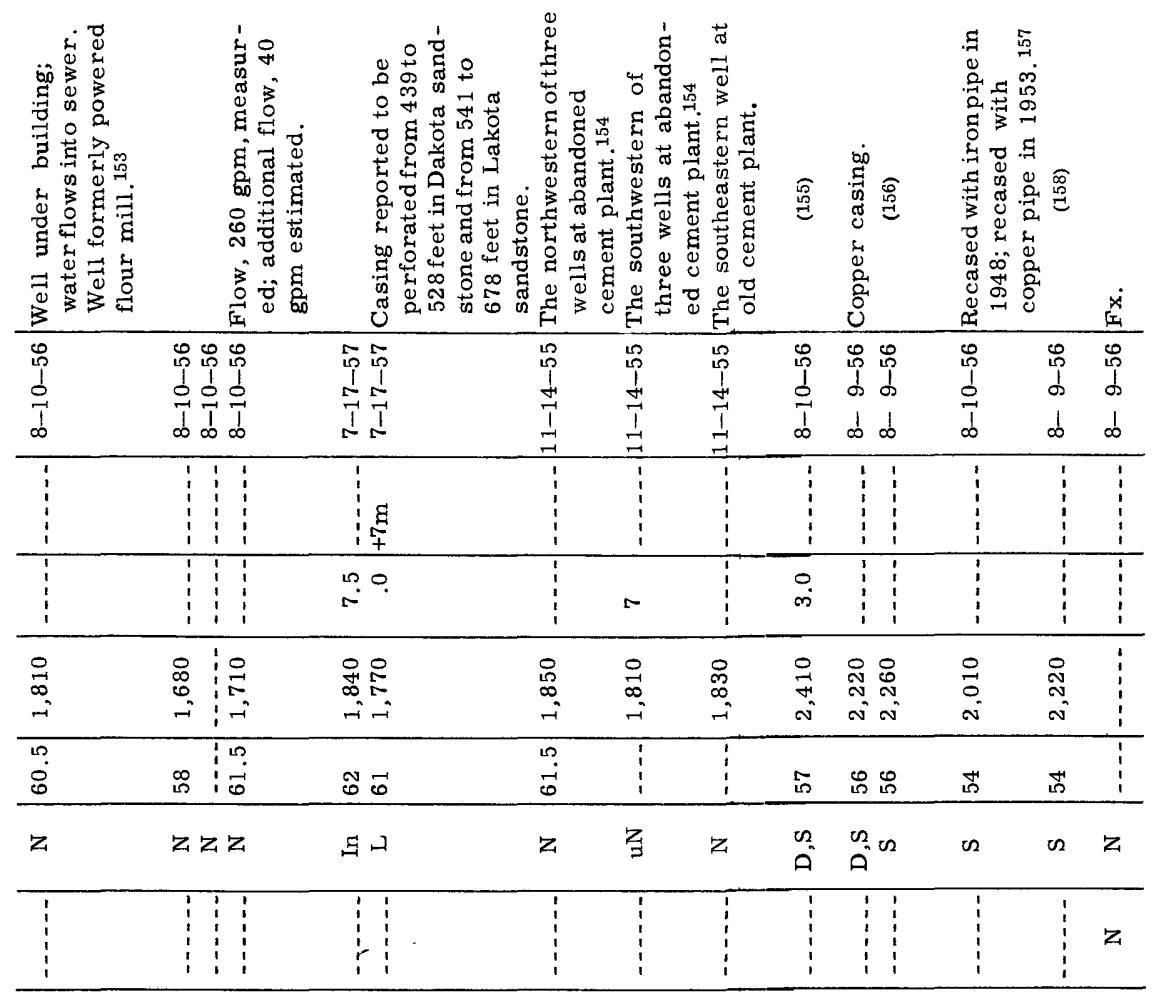

\begin{tabular}{|c|c|c|c|c|c|c|c|c|c|c|}
\hline $\begin{array}{l}\underline{g} \\
0 \\
\stackrel{-}{-1} \\
\text { E. }\end{array}$ & 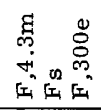 & 㞓 & $\begin{array}{l}\text { घ: } \\
\infty \\
\infty \\
\underbrace{-}\end{array}$ & 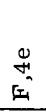 & 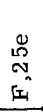 & 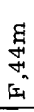 & 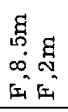 & 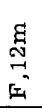 & 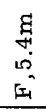 & $z$ \\
\hline 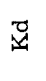 & 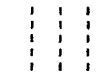 & $\begin{array}{l}\vec{x} \\
\vec{x}\end{array}$ & 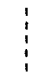 & 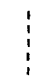 & 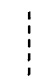 & 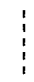 & 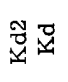 & ָै & $\overrightarrow{0}$ & 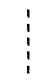 \\
\hline
\end{tabular}

\begin{tabular}{|c|c|c|c|c|c|c|c|c|c|c|}
\hline$\infty$ & 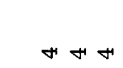 & $\begin{array}{ll}5 & 0 \\
\infty & 0 \\
0 & \end{array}$ & $\infty$ & $\infty$ & 0 & $H$ & 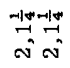 & N & ๓ & \\
\hline$\stackrel{\infty}{\circ}$ & 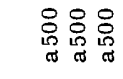 & जै & 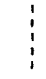 & 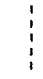 & 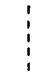 & $\vdots$ & 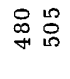 & $\begin{array}{l}\infty \\
\underset{\sim}{*}\end{array}$ & 怘 & \\
\hline $\begin{array}{l}\text { No } \\
\infty \\
\infty \\
-1\end{array}$ & 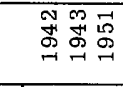 & $\begin{array}{l}\text { 吾品 } \\
\text { 品 }\end{array}$ & $\begin{array}{l}1 \\
\vdots \\
\vdots \\
\vdots\end{array}$ & 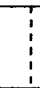 & 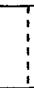 & $\begin{array}{l}1 \\
\vdots \\
\vdots\end{array}$ & 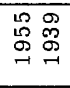 & 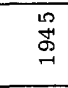 & 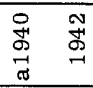 & $\frac{1}{3}$ \\
\hline $\begin{array}{l}\vdots \\
\vdots \\
0 \\
\vdots \\
0 \\
0 \\
0 \\
0 \\
0 \\
\text { है }\end{array}$ & 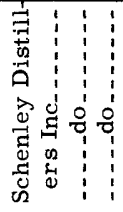 & 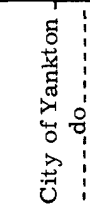 & 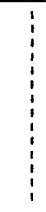 & $\begin{array}{c}\vdots \\
\vdots \\
\vdots \\
\vdots\end{array}$ & 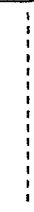 & 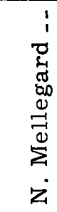 & 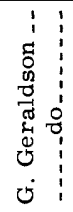 & 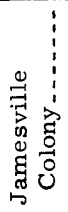 & 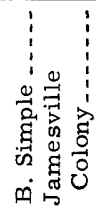 & $\begin{array}{ll}\vdots \\
\vdots \\
0 \\
0 \\
0 \\
0 \\
0 \\
0 \\
0 \\
0 \\
0 \\
0 \\
0 \\
0\end{array}$ \\
\hline $\begin{array}{l}\infty \\
\infty \\
-1\end{array}$ & $\begin{array}{lll}\ddot{H} & 0 & 0 \\
0 & 0 & 0 \\
-1 & \infty & 0\end{array}$ & 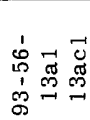 & స్ & స్ & 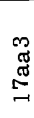 & 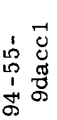 & 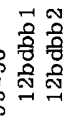 & $\begin{array}{l}\vec{z} \\
\tilde{d} \\
0 \\
0 \\
\text { N } \\
\text { N }\end{array}$ & $\begin{array}{l}\overrightarrow{0} \\
\stackrel{0}{0} \\
\stackrel{0}{0} \\
\stackrel{0}{0} \\
\stackrel{N}{N}\end{array}$ & o \\
\hline
\end{tabular}




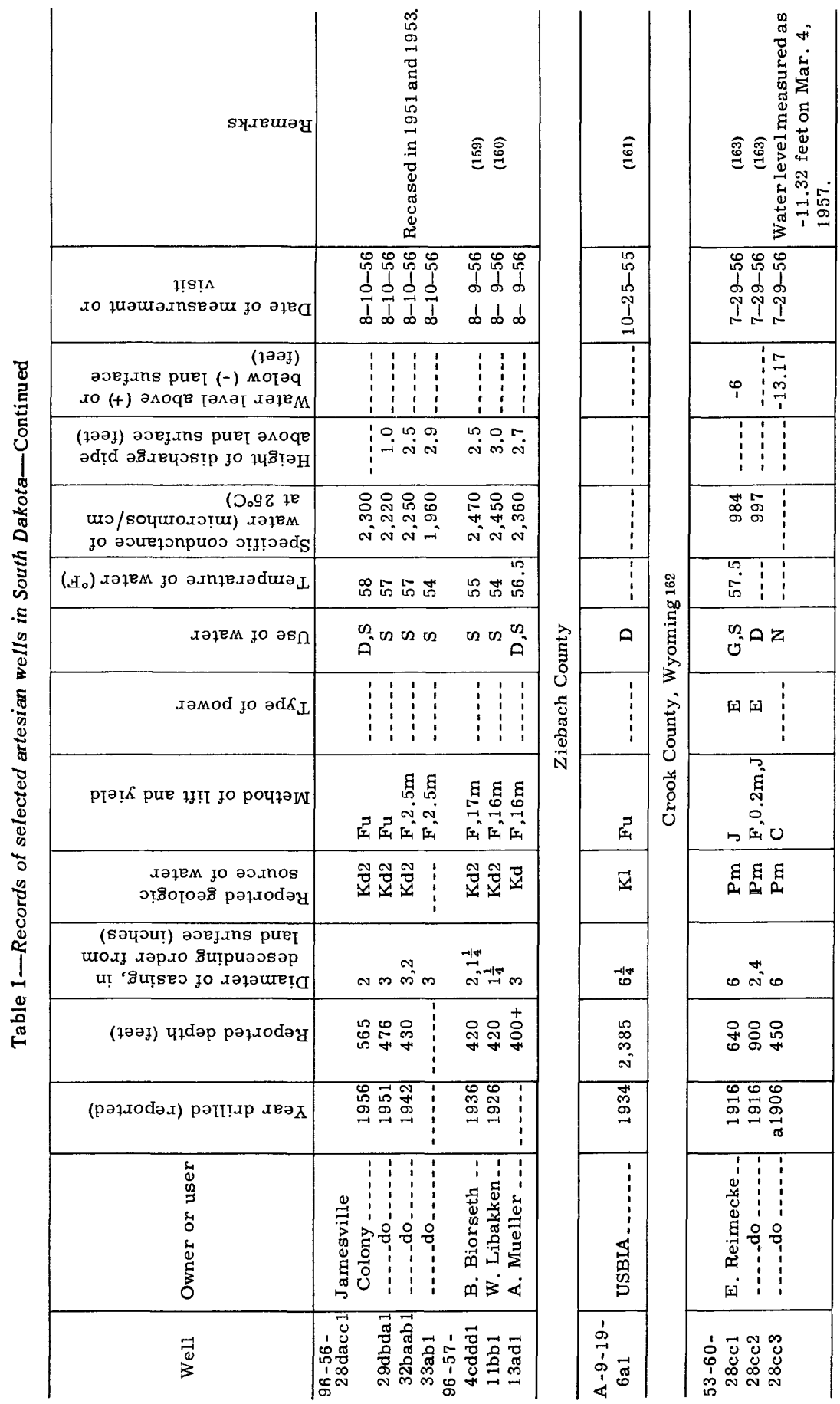



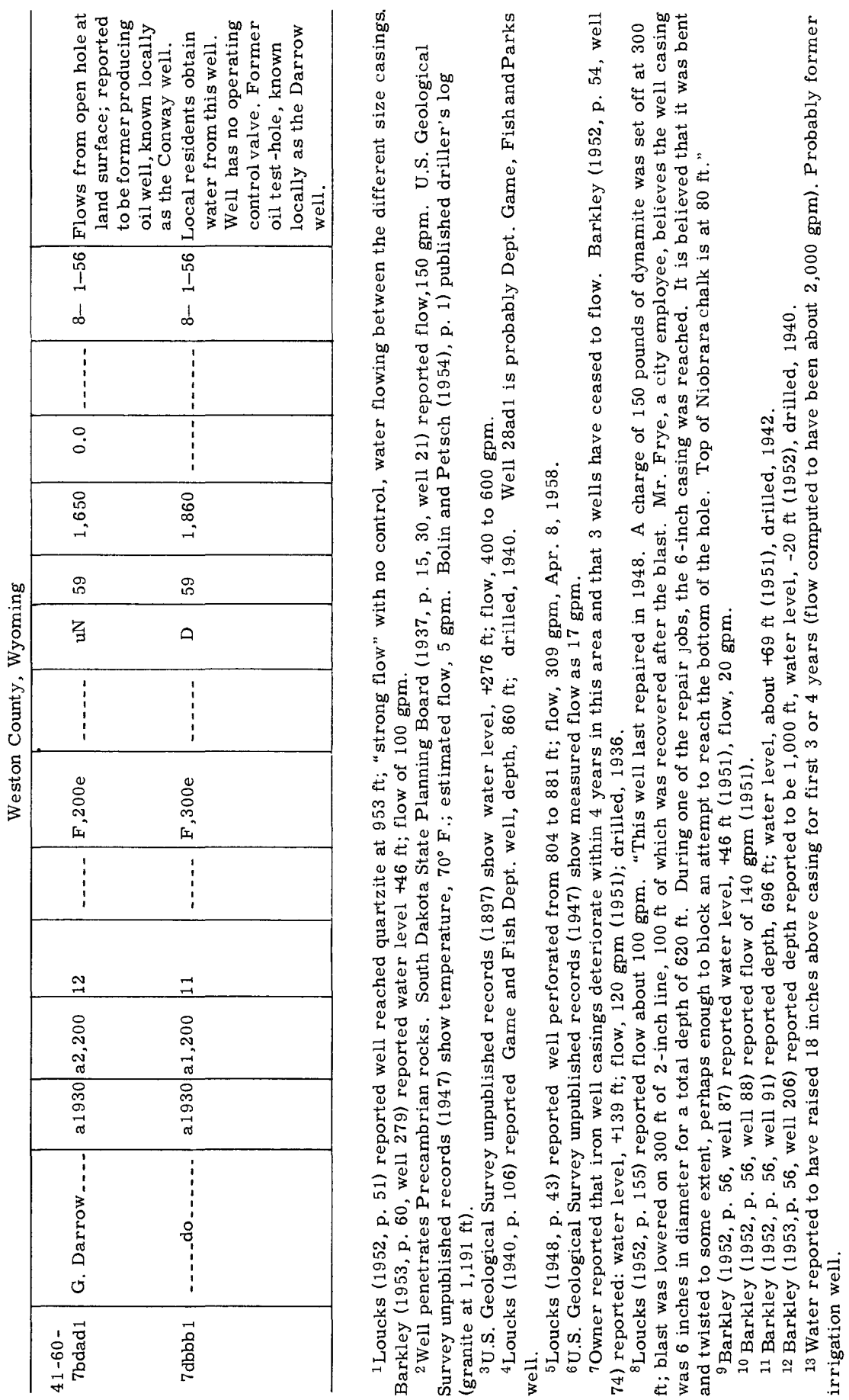

553972 O-61-7 


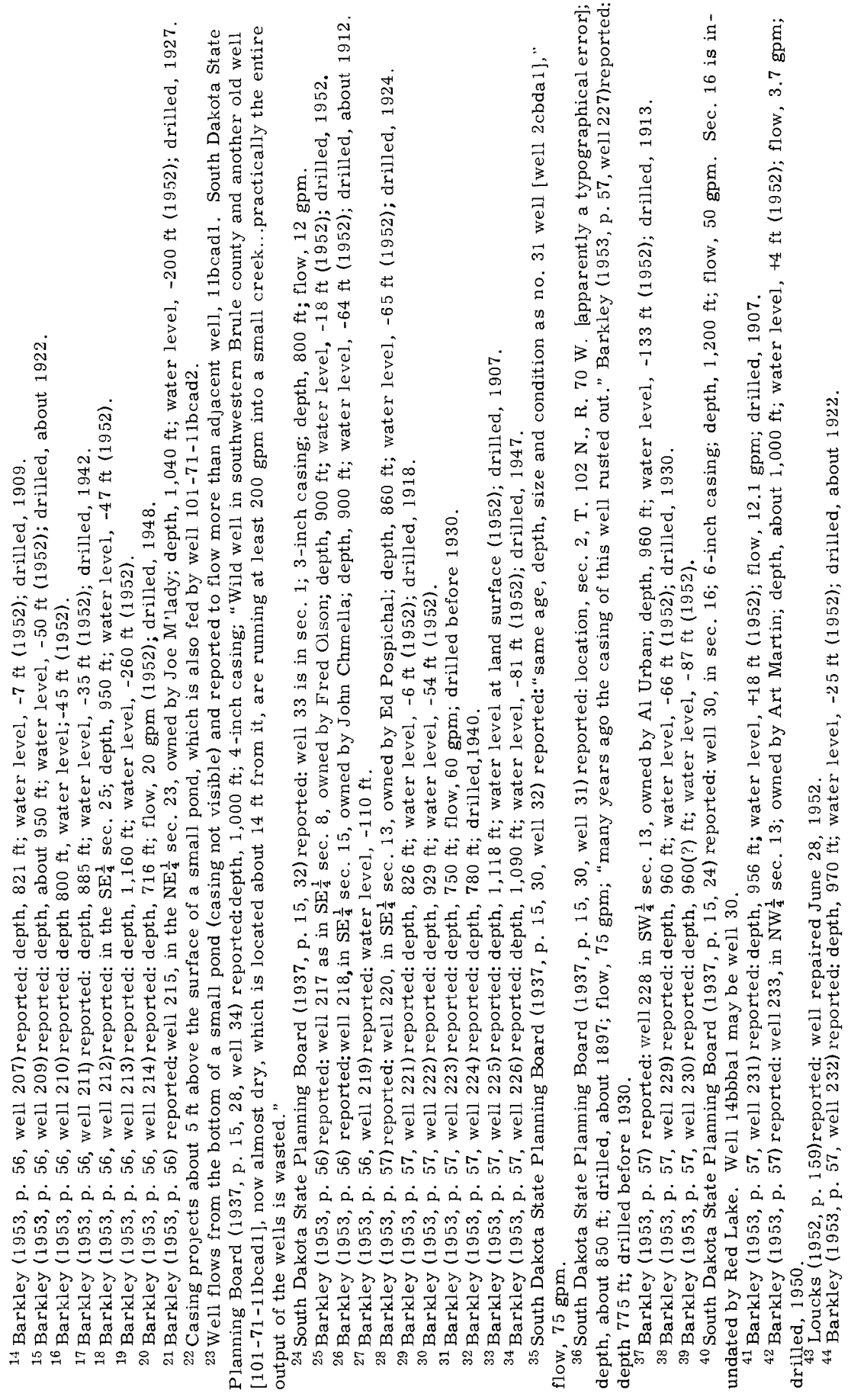




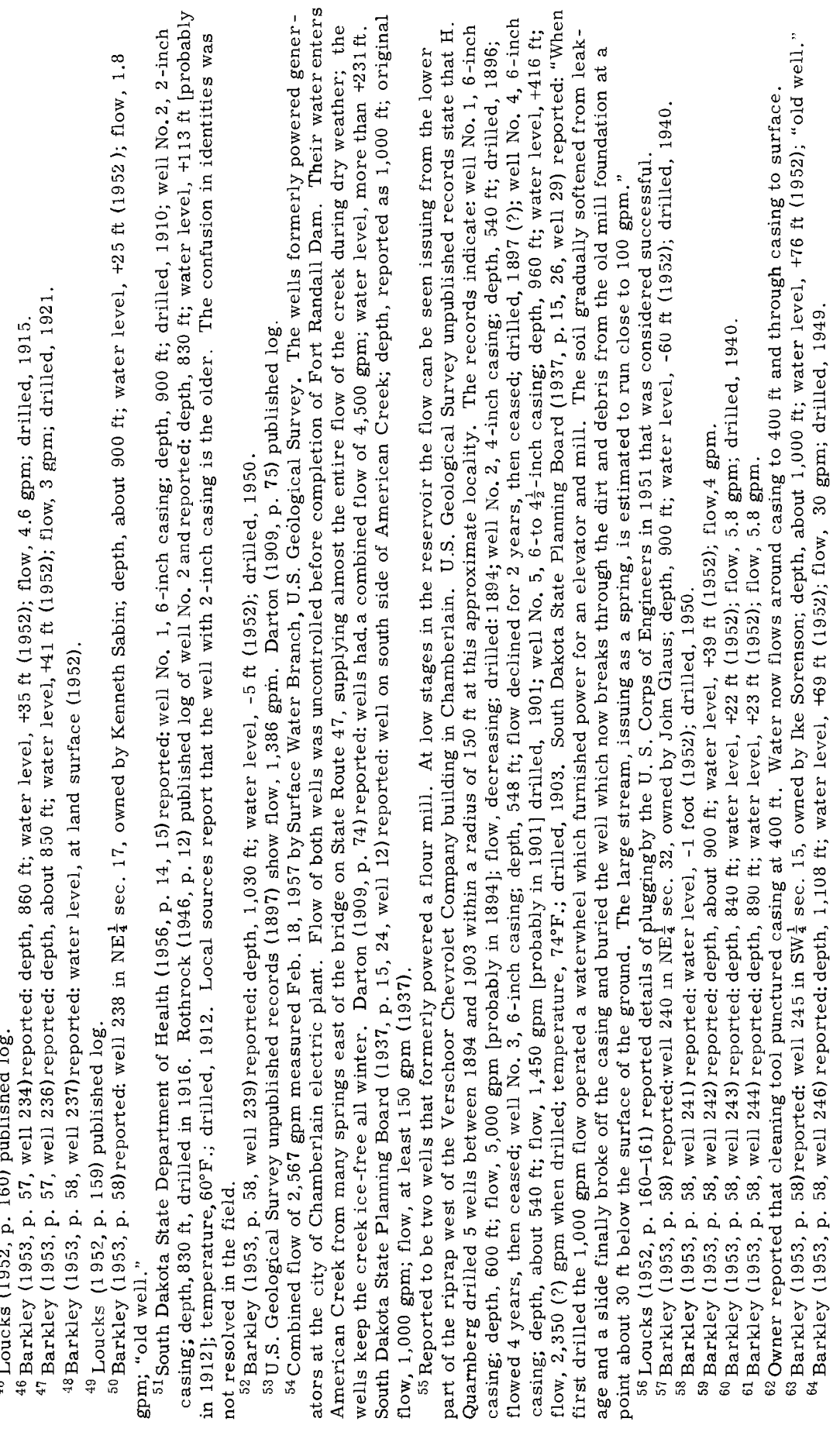



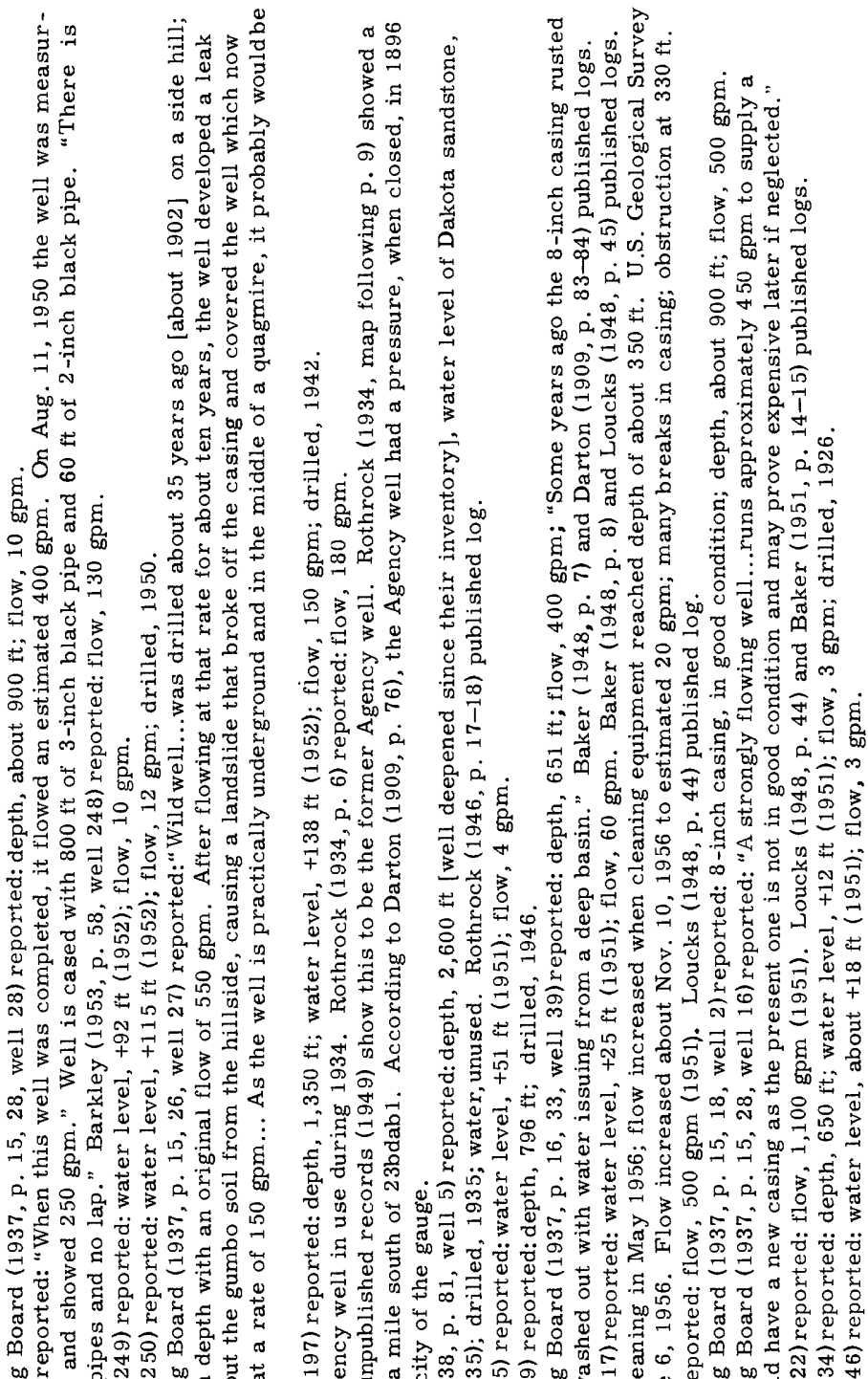

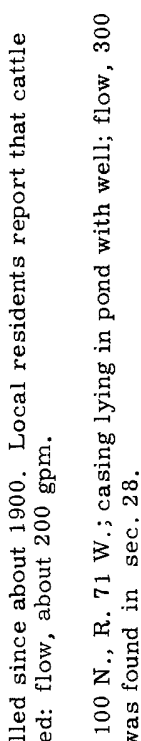

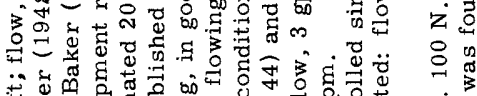
के 要

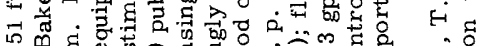

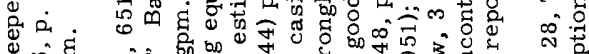
षै

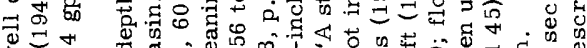

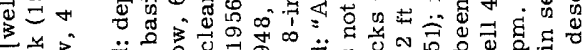

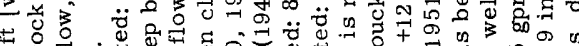

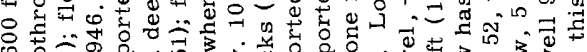

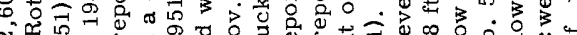
N 0 की

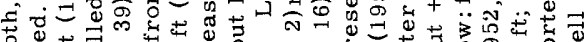

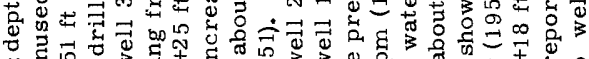

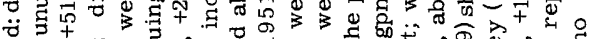

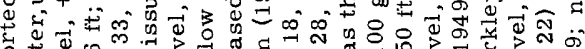

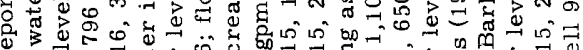
चे वे क्ष

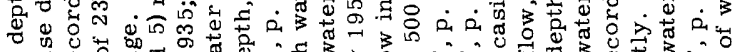

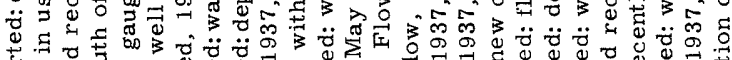

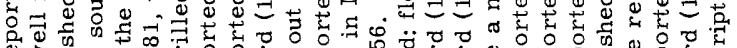

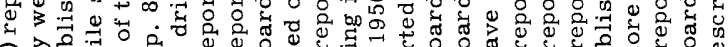

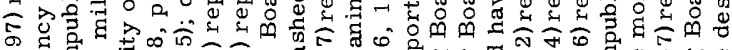
कo

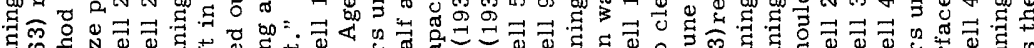

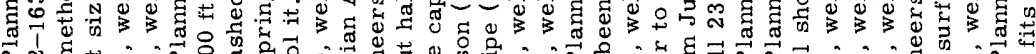

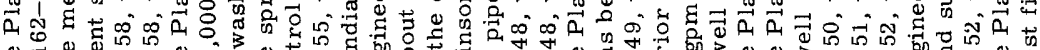

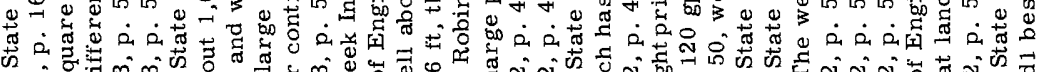

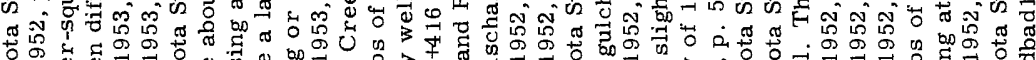

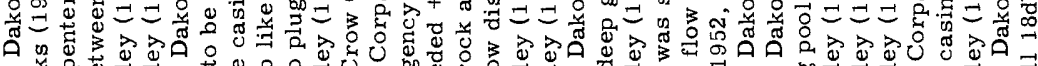

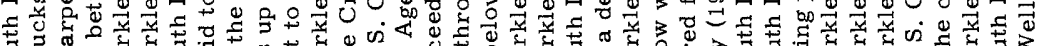

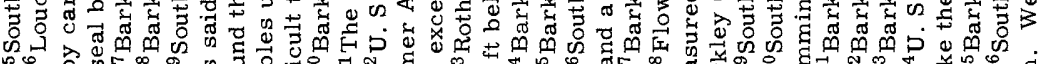

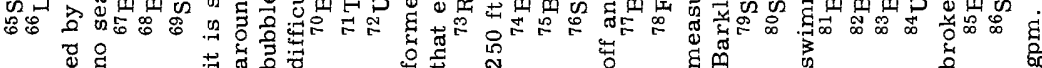




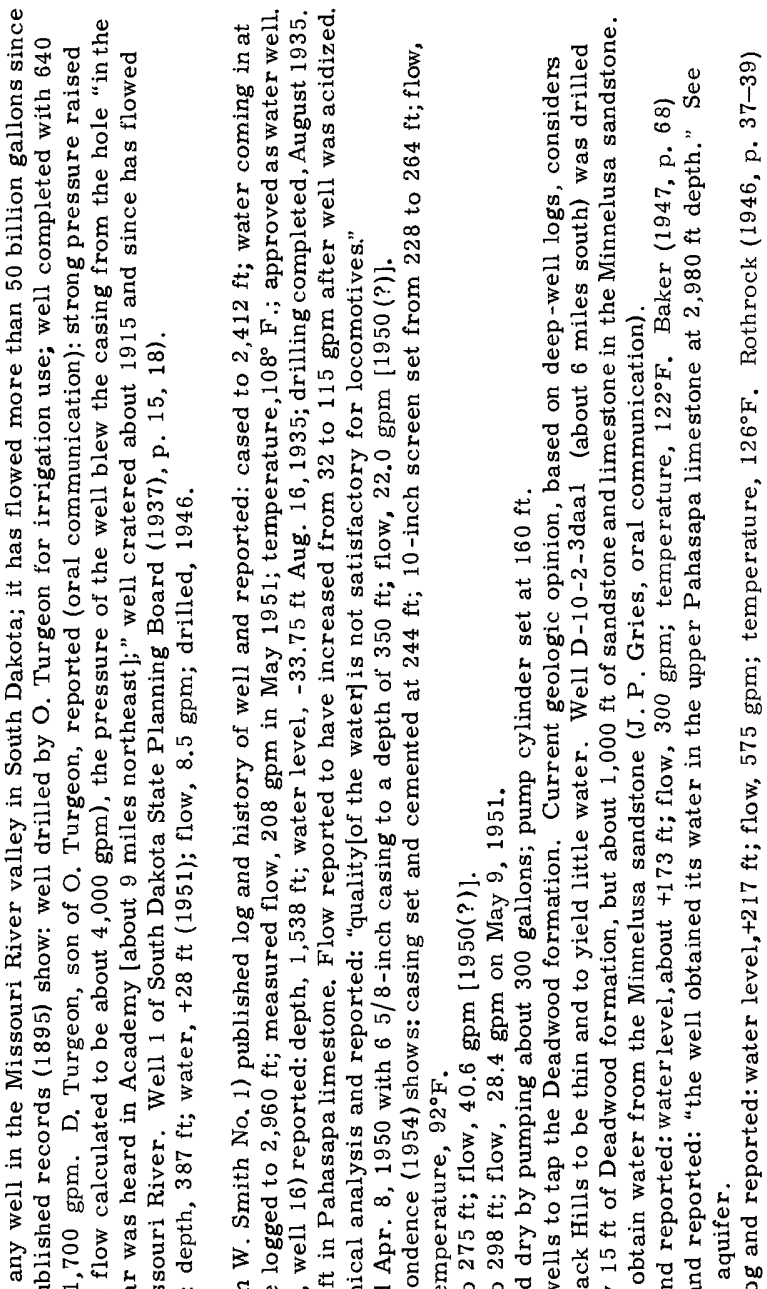

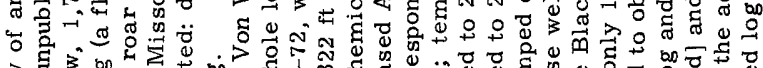

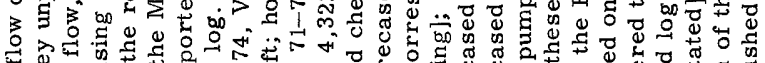

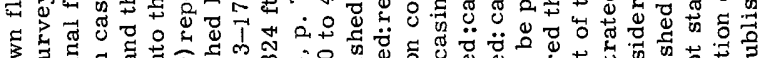

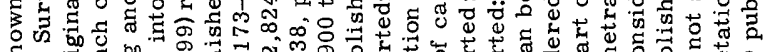

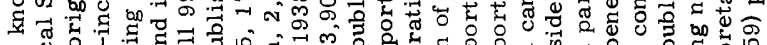

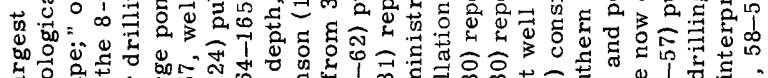

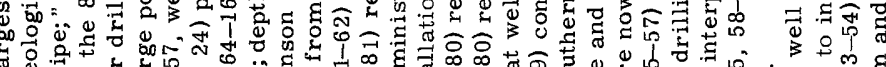

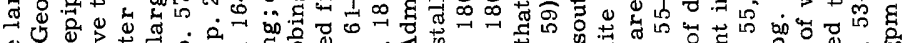
Q † ต

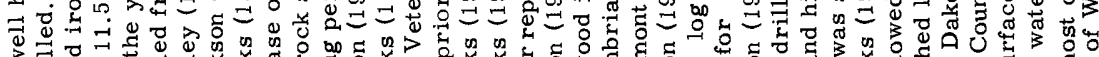

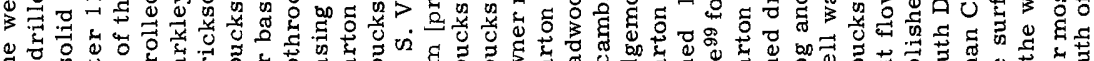

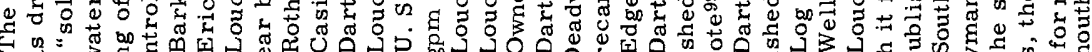
E 心 


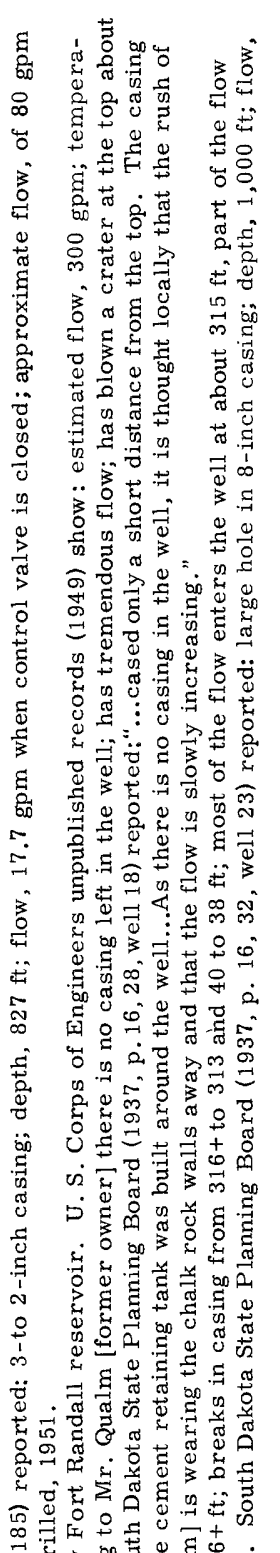

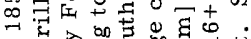

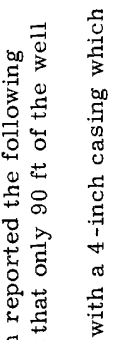

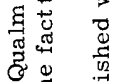

$\therefore$ 娄

$\begin{array}{cc}0 \\ -1 & 0 \\ 0 & 0\end{array}$

छี

वृ.

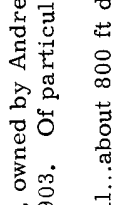

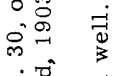

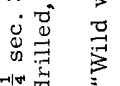

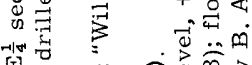

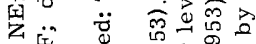

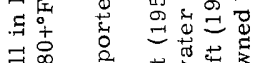

700

药

运苟

ङे छें

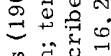

on

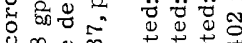

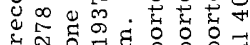

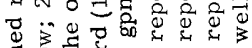
击

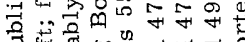

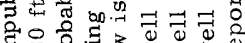

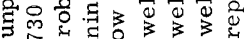

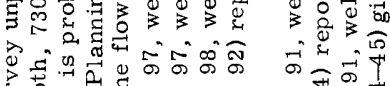

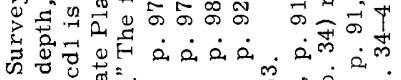

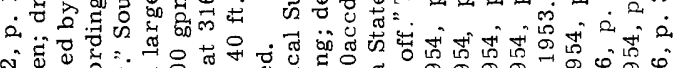
N

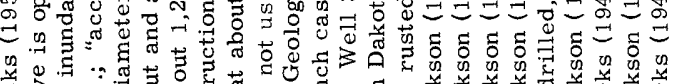

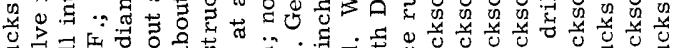

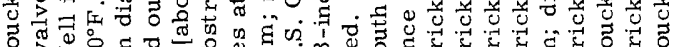

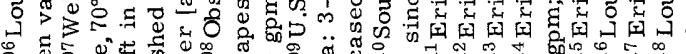

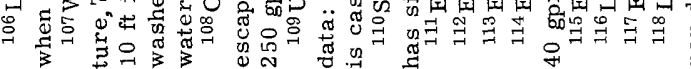

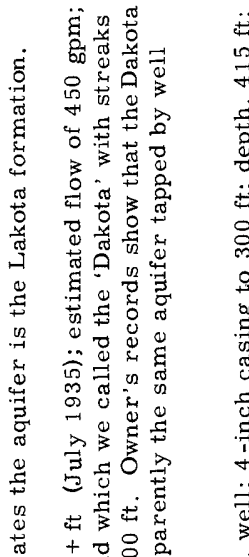

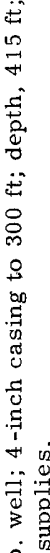

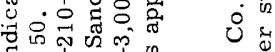

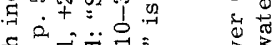
- $\overrightarrow{0} \overrightarrow{0} \ddot{0} \overrightarrow{0}$ $=$ \&

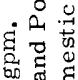

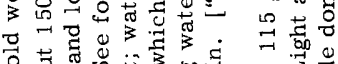

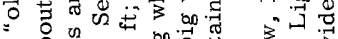

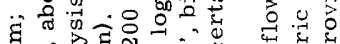
कृ 0.0

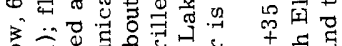

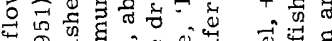

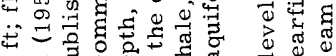

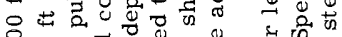

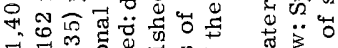

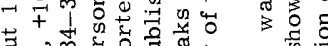
चे ले

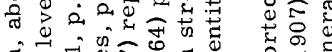

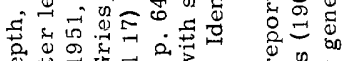

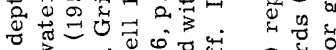

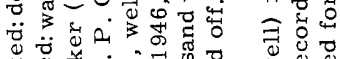

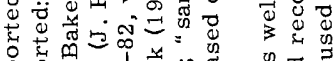

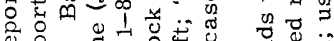
\& क人

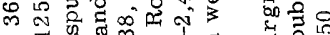

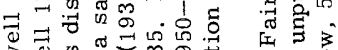

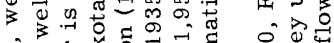

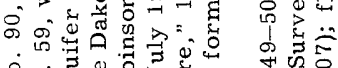

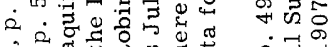
तi

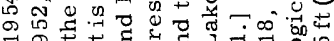
$=$ 击

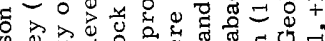
w

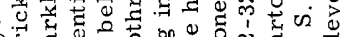

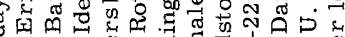

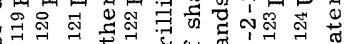




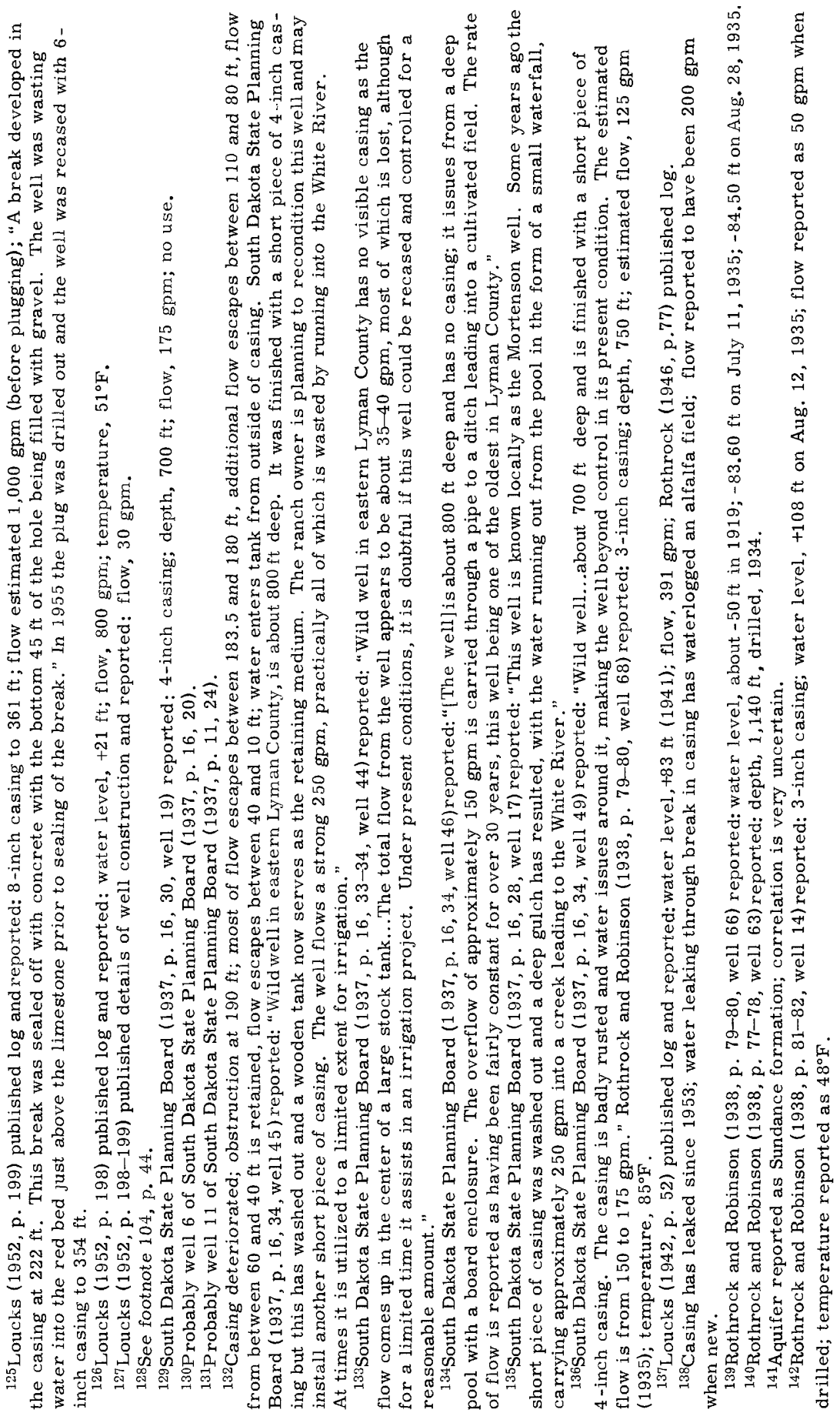




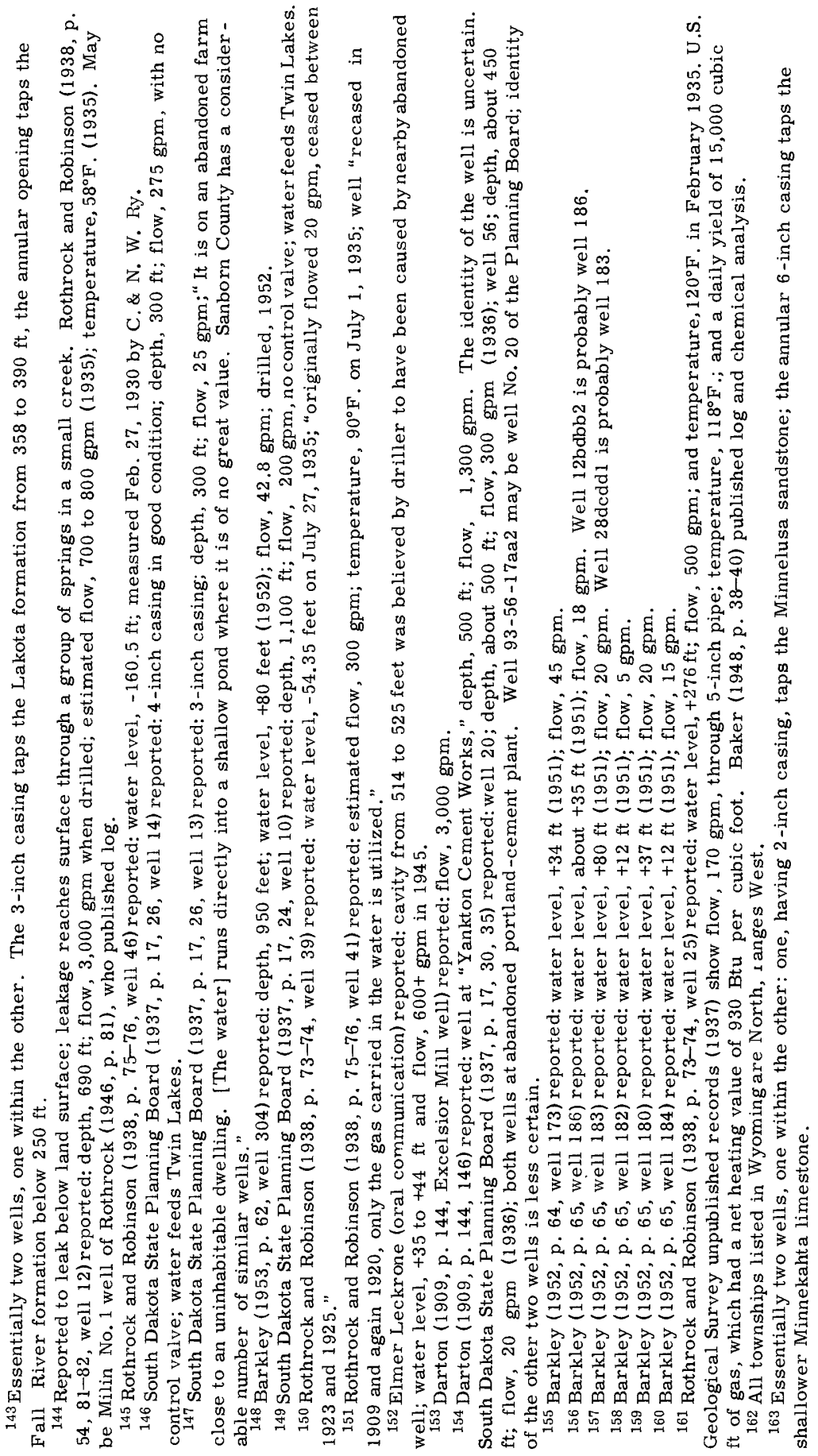







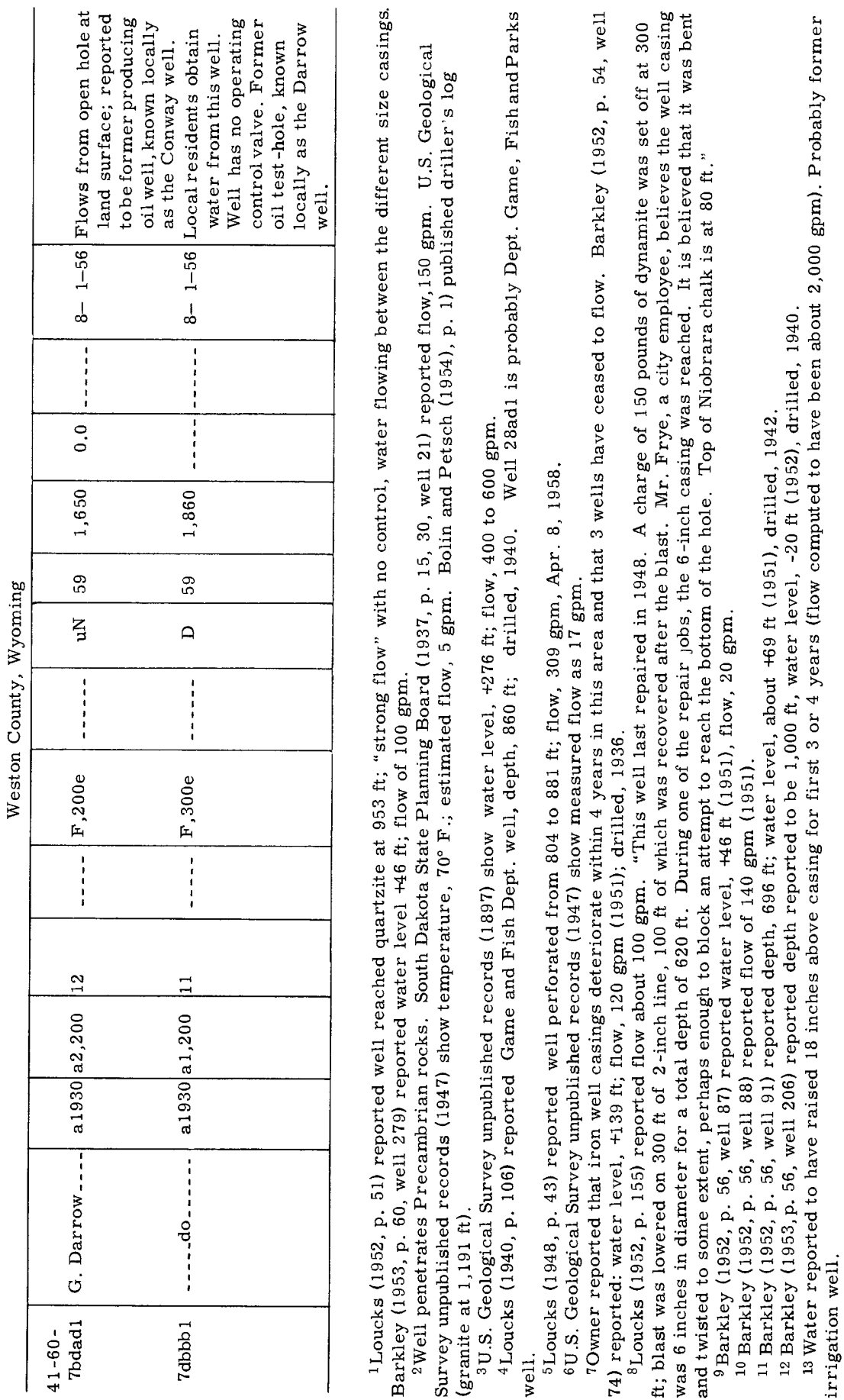

553972 O-61-7 


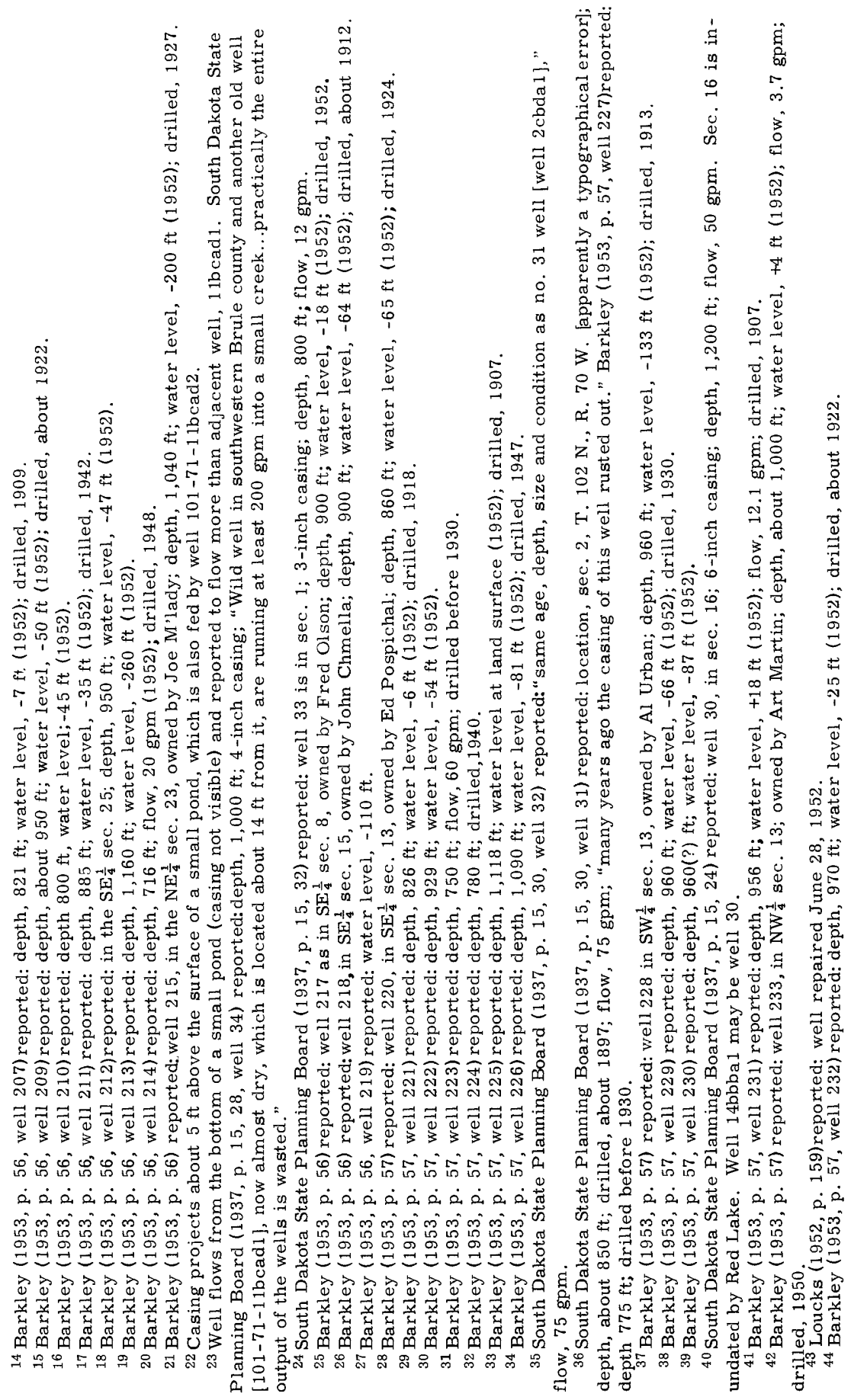




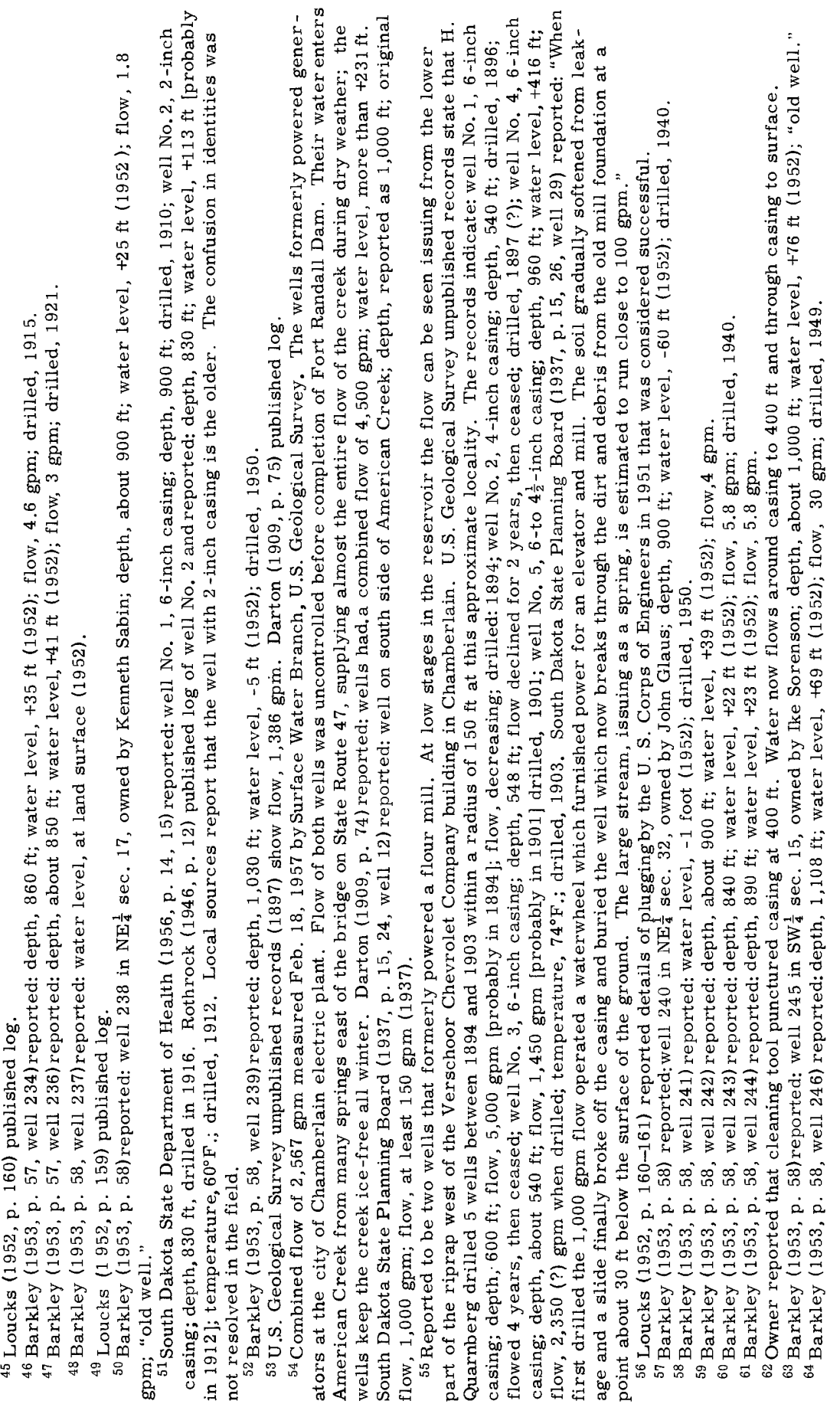



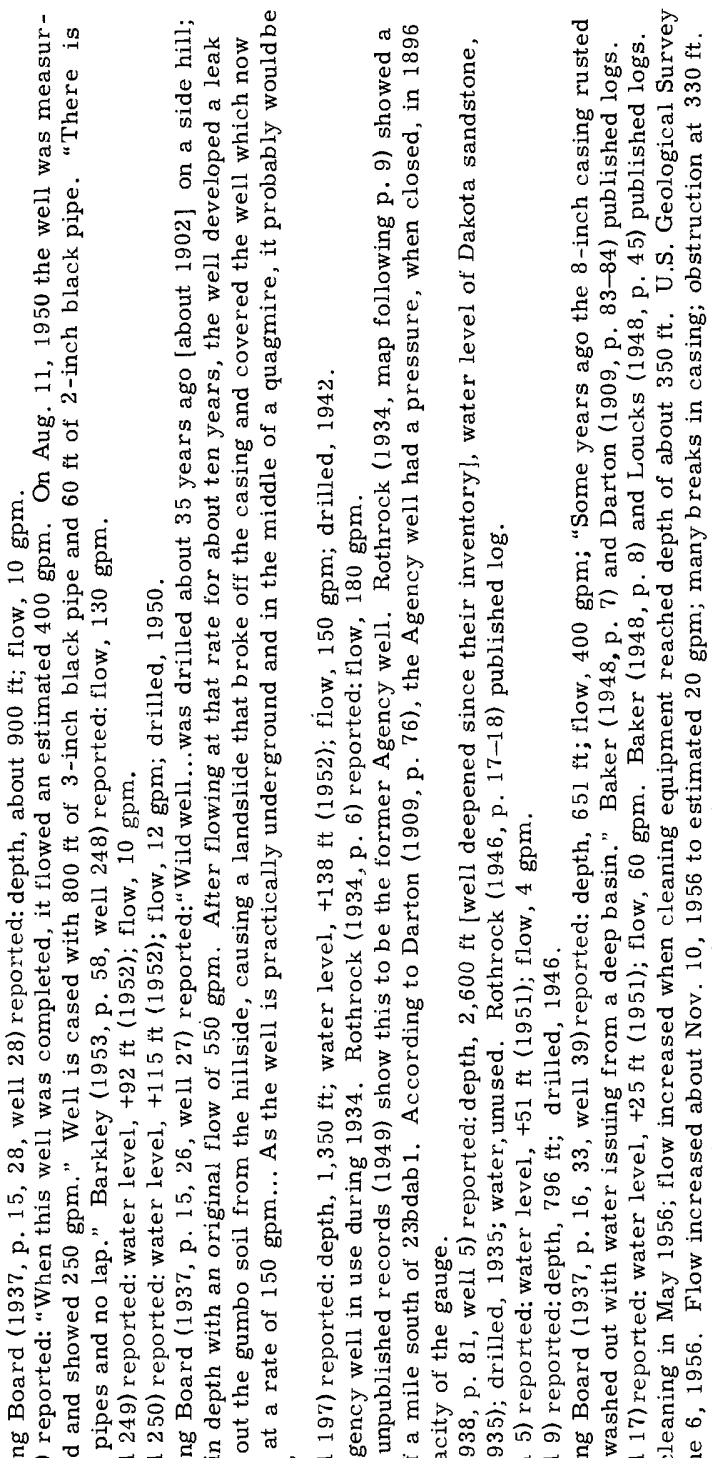

का

$\sum_{\infty}^{\infty} 20$ ०

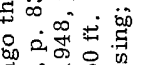

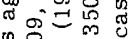

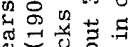

$D$ वे

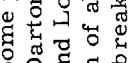
ถั่

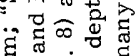

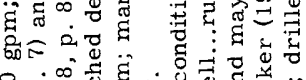

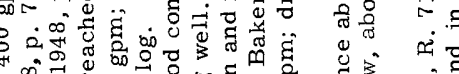

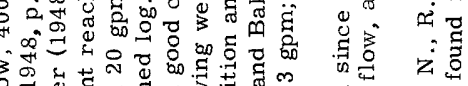

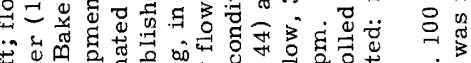

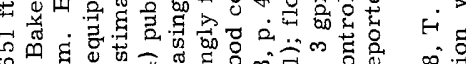

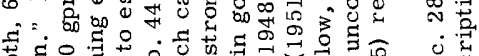
营.

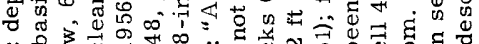
تं

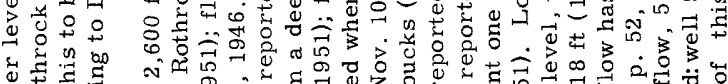

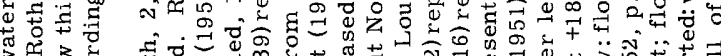

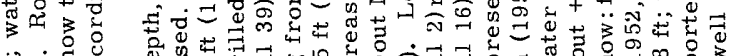

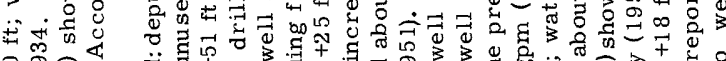

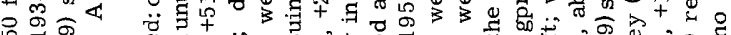

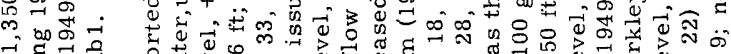

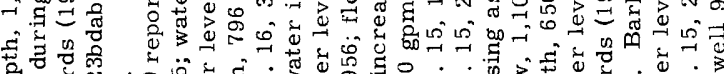

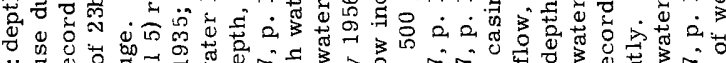

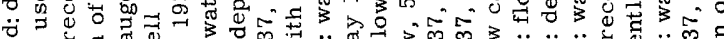

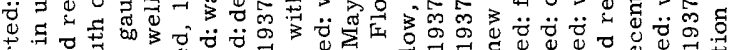

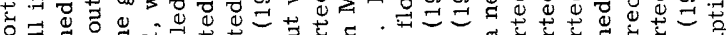
बे की

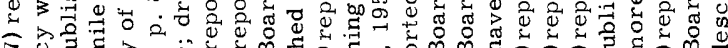

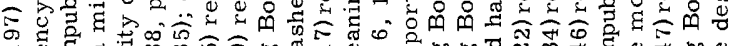

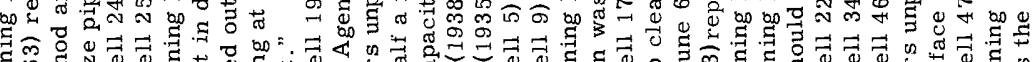

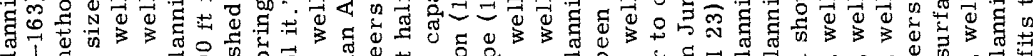
जิ

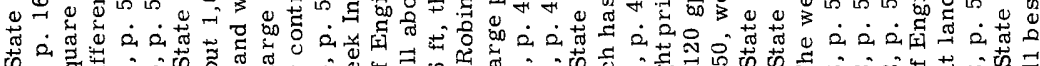

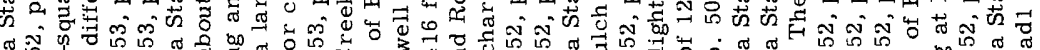

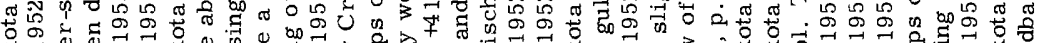
急

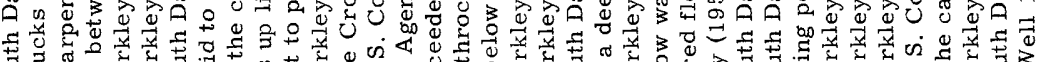
च च 论

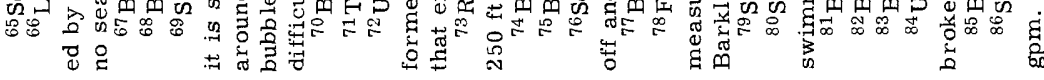




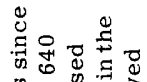

设

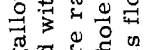

00

茟设

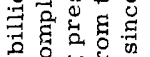

$\circ 8$ so 20

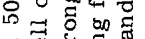

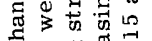

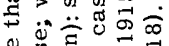

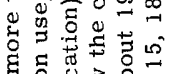

द

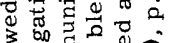

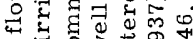

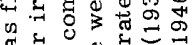
도

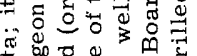

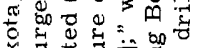

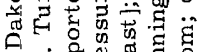
ᄃ 0 \% 造造

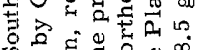
ध > व $\Rightarrow$ क्ष त)

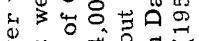

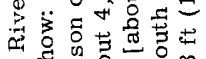
$\rightarrow$ 的证

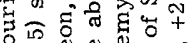
on o 0 :

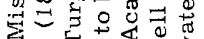
व

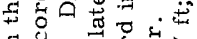

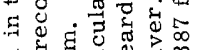

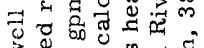

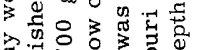

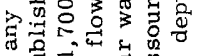

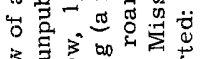

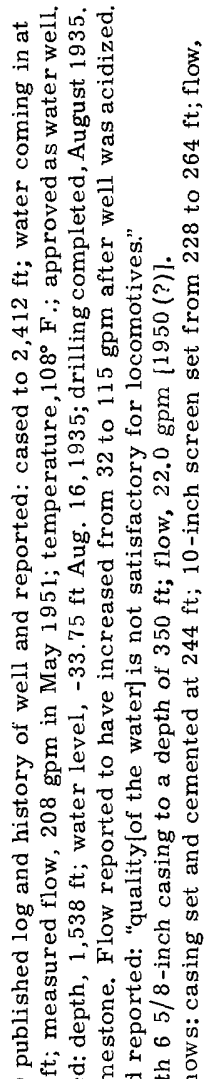

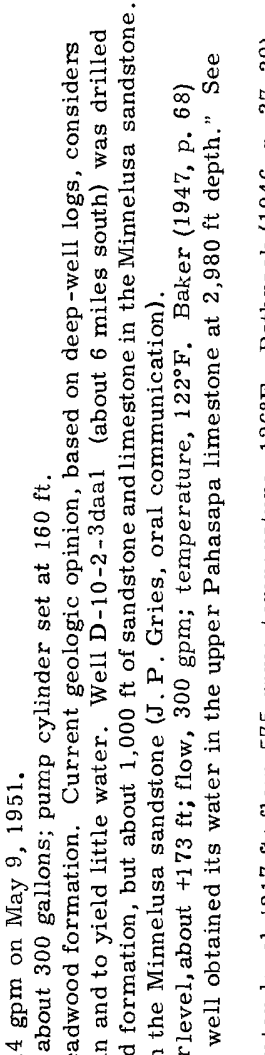

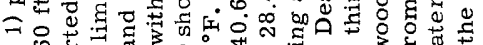

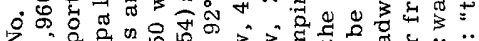

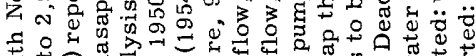

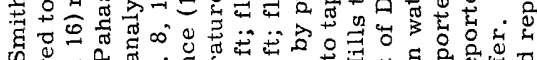

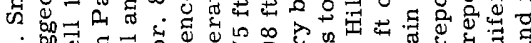

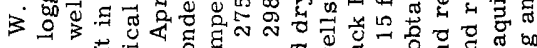
ปี

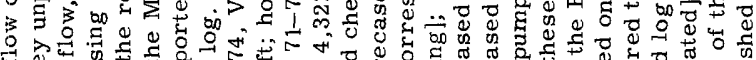

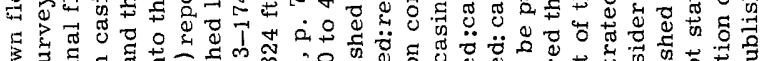

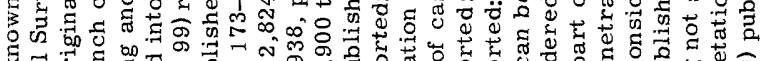

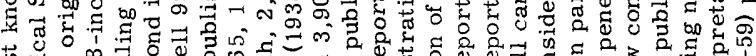

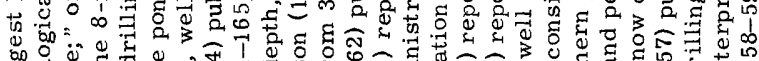

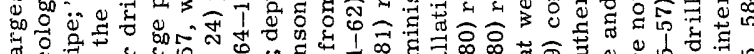

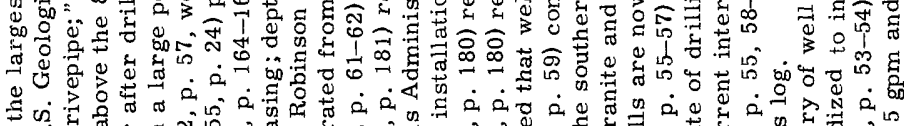

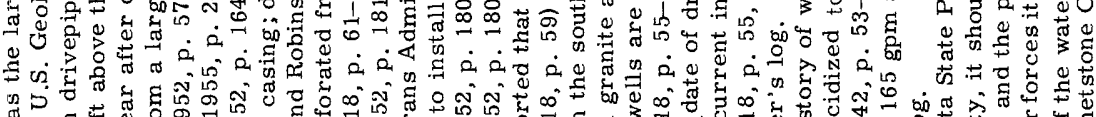

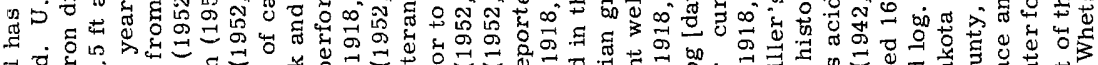

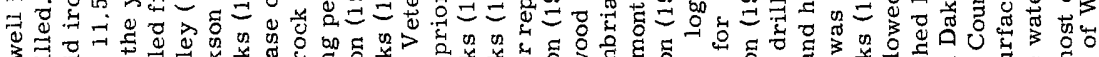

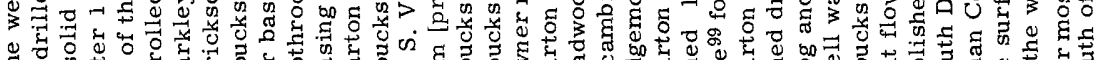
I $H$ W 350

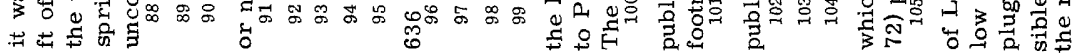




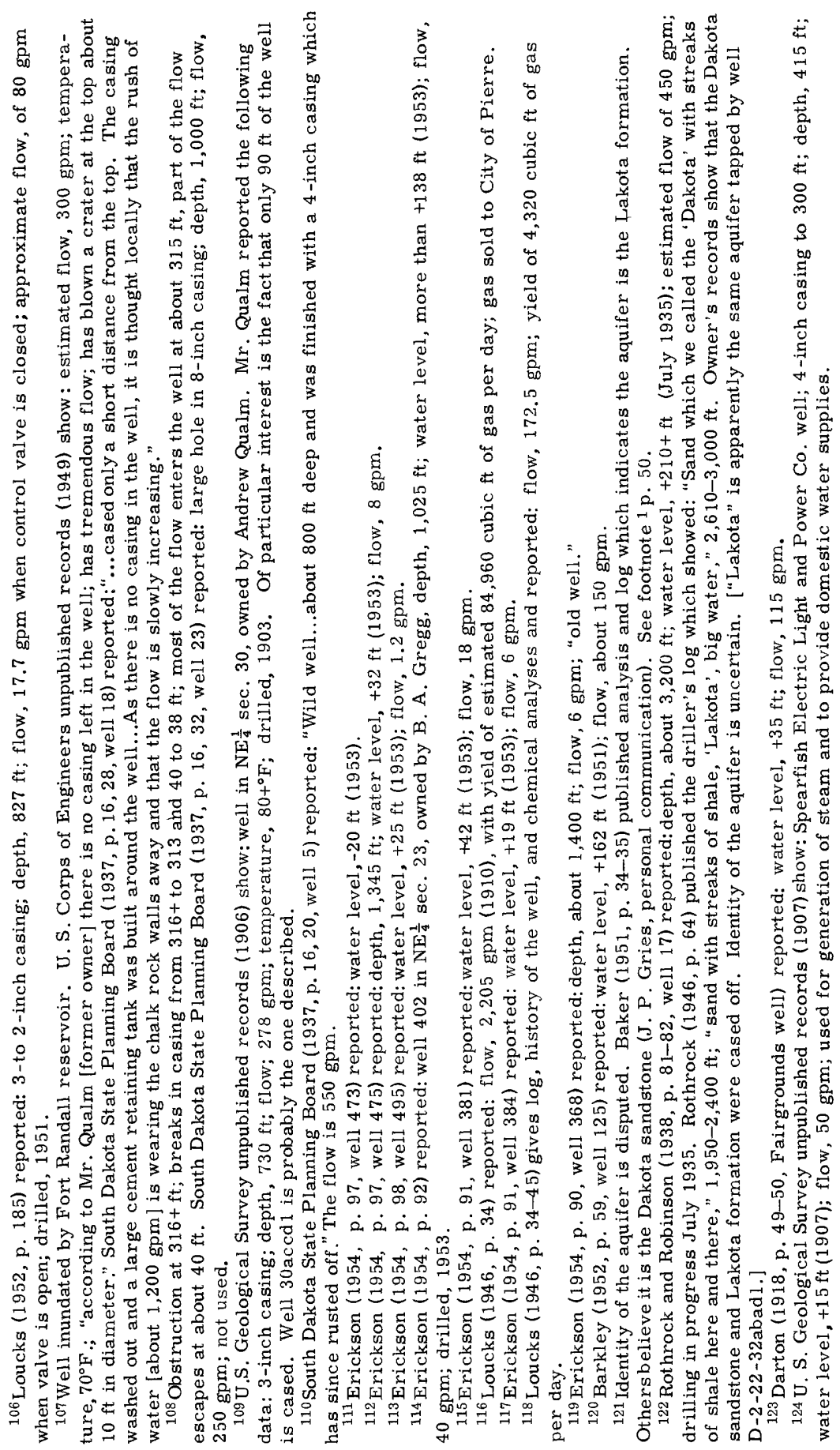




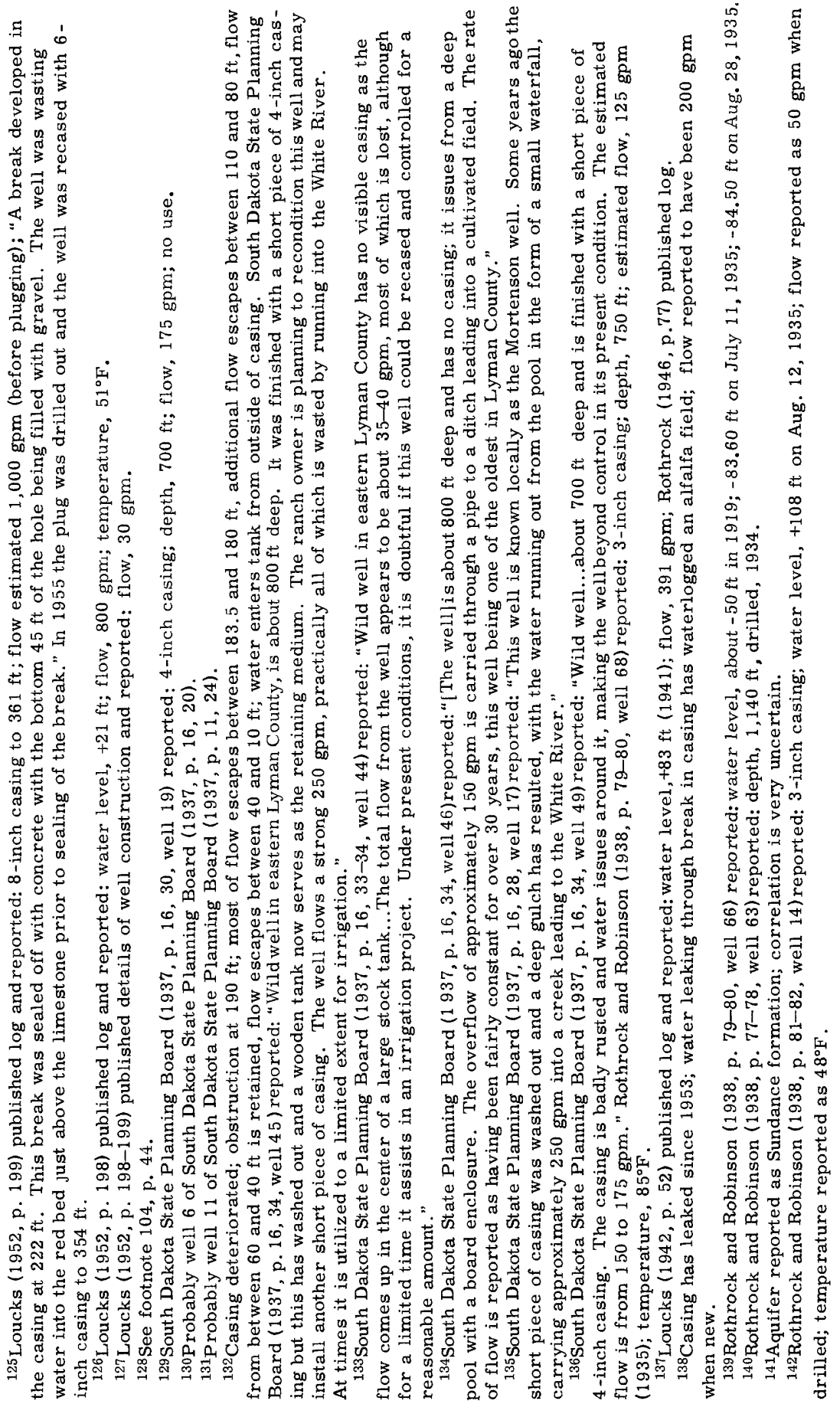




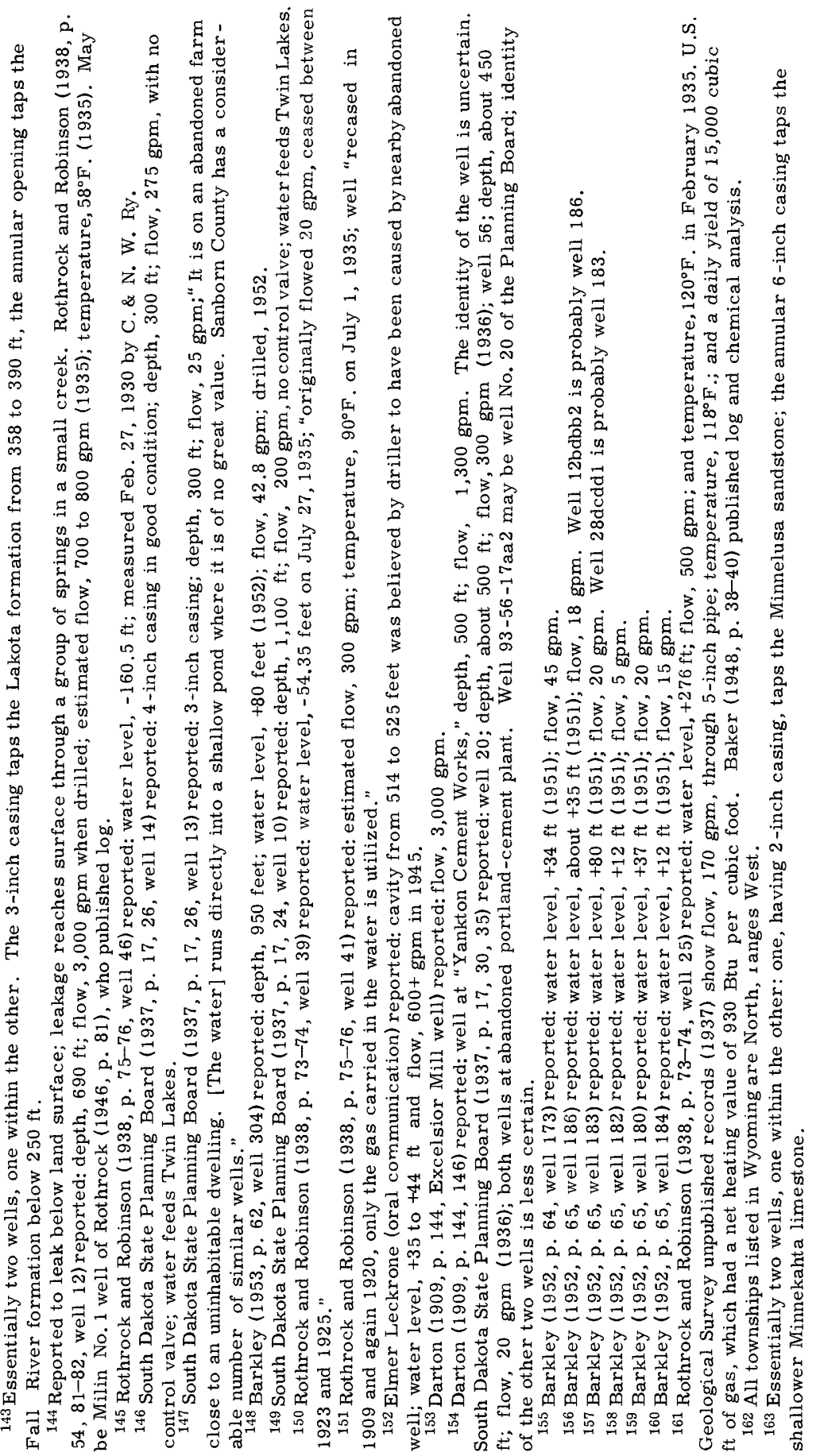





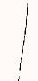

